Distributions of 14 Elements on 63 Absorbers from Three Simulant Solutions (Acid-Dissolved Sludge, Acidified Supernate, and Alkaline Supernate) for Hanford HLW Tank 102-SY

S. Fredric Marsh*

Zita V. Svitra

Scott M. Bowen 


\section{DISCLAIMER}

This report was prepared as an account of work sponsored by an agency of the United States Government. Neither the United States Government nor any agency thereof, nor any of their employees, make any warranty, express or implied, or assumes any legal liability or responsibility for the accuracy, completeness, or usefulness of any information, apparatus, product, or process disclosed, or represents that its use would not infringe privately owned rights. Reference herein to any specific commercial product, process, or service by trade name, trademark, manufacturer, or otherwise does not necessarily constitute or imply its endorsement, recommendation, or favoring by the United States Government or any agency thereof. The views and opinions of authors expressed herein do not necessarily state or reflect those of the United States Government or any agency thereof. 


\section{DISCLAIMER}

Portions of this document may be illegible in electronic image products. Images are produced from the best available original document. 


\section{CONTENTS}

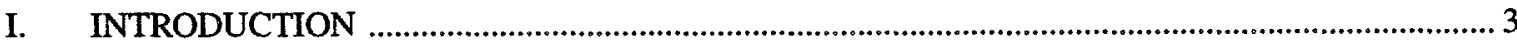

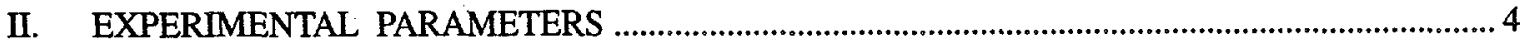

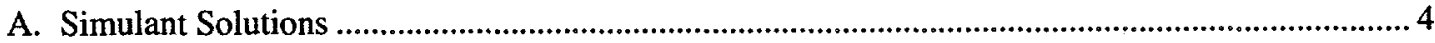

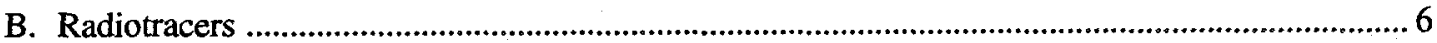

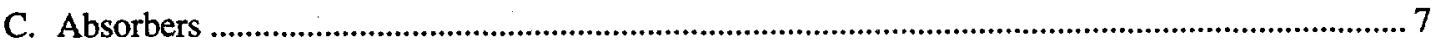

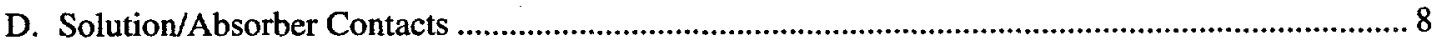

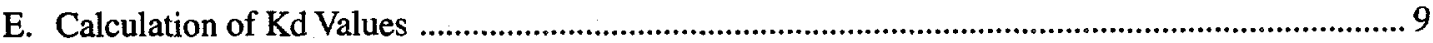

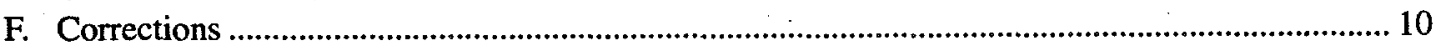

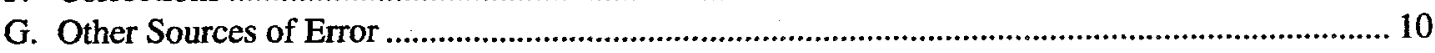

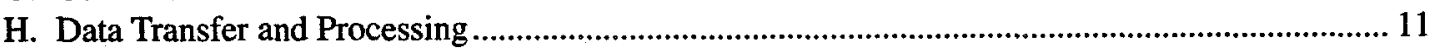

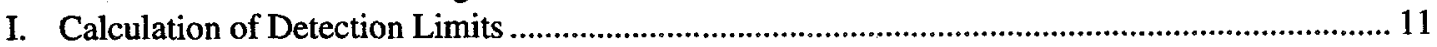

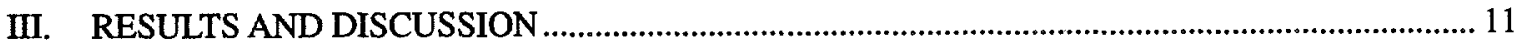

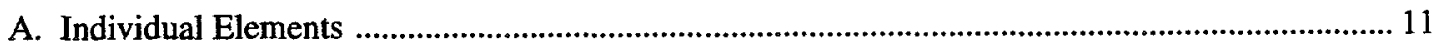

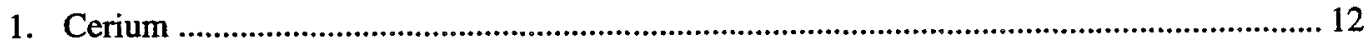

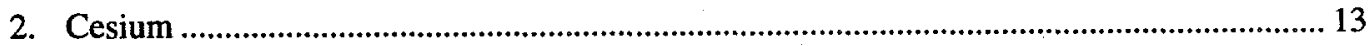

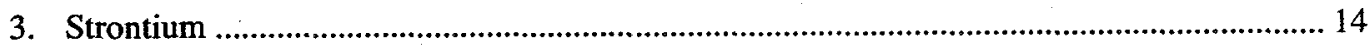

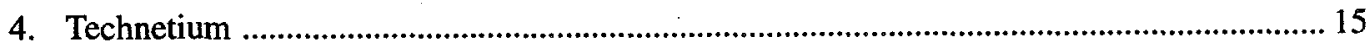

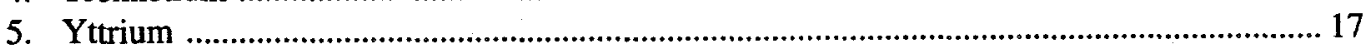

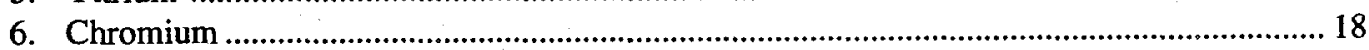

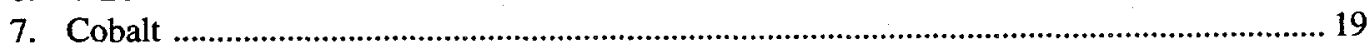

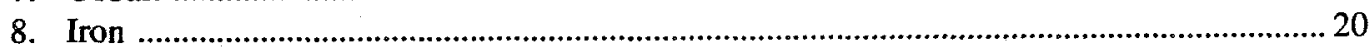

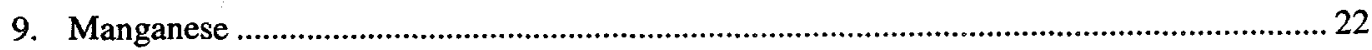

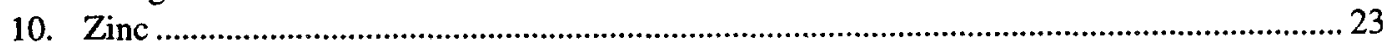

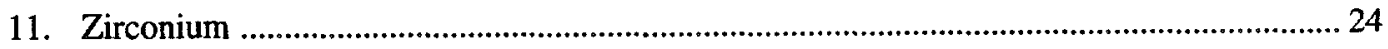

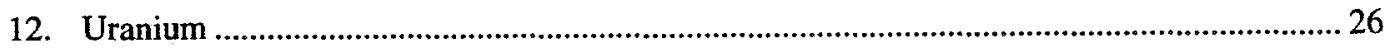

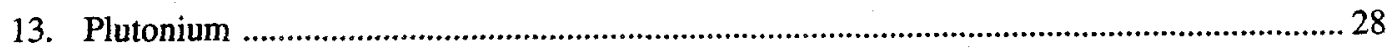

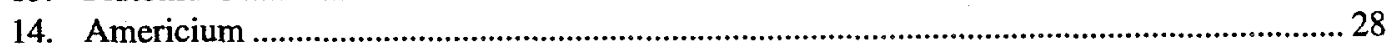

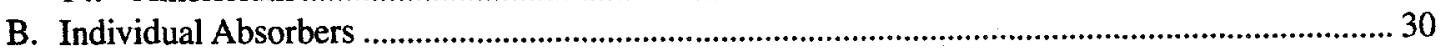

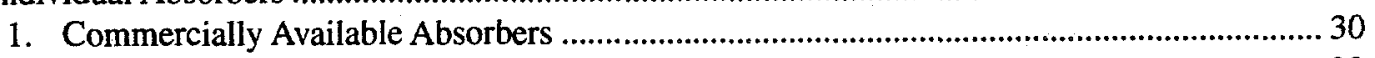

a. Amberlyst ${ }^{\mathrm{TM}} 15$ Cation Exchange Resin ...................................................................... 30

b. Amberlyst ${ }^{\mathrm{TM}} \mathrm{XN}-1010$ Cation Exchange Resin ............................................................ 32

c. Duolite ${ }^{\mathrm{TM}} \mathrm{C}-467$ Cation Exchange Resin ………............................................................... 33

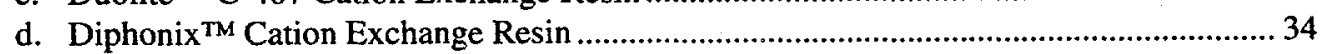

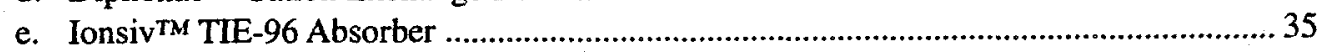

f. Ionsiv TM TIE-96 (Modified) Absorber .............................................................................. 36

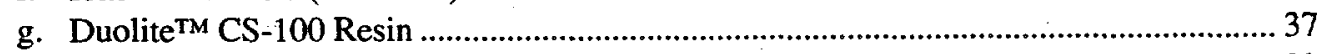

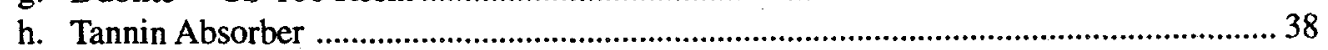

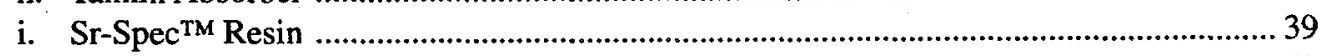

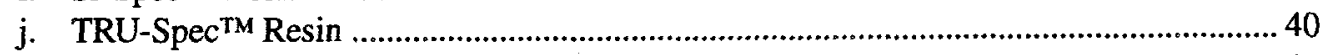

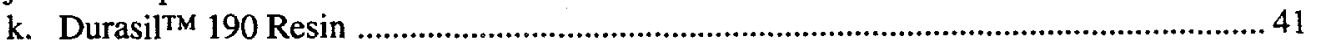

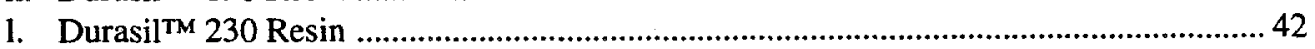

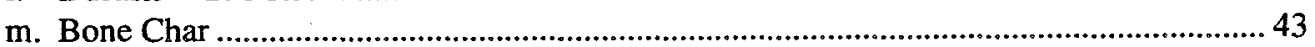




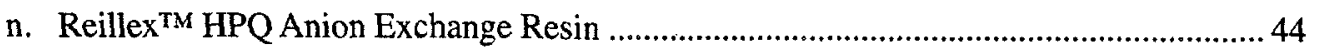

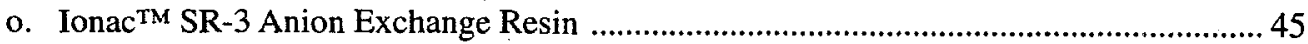

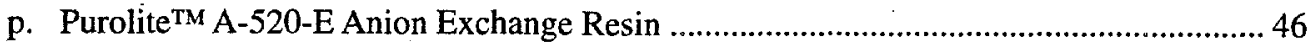

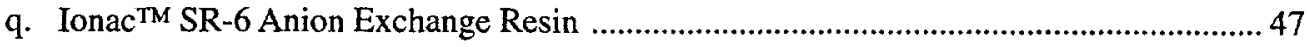

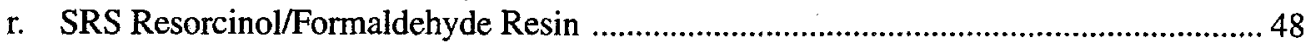

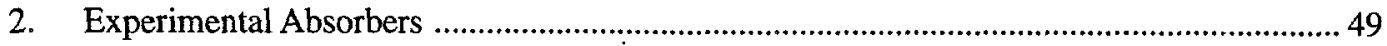

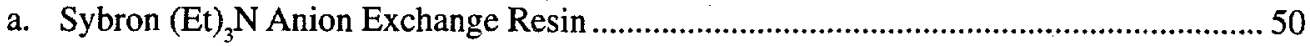

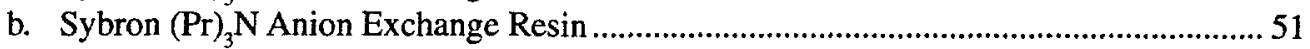

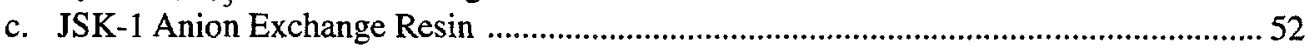

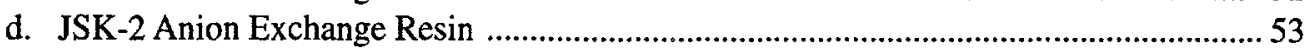

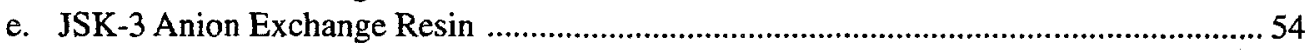

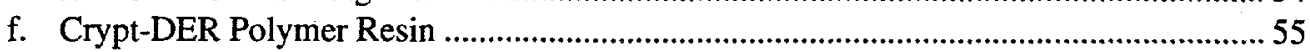

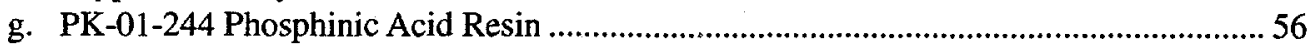

3. Polyacrylonitrile (PAN) Composite Absorbers ................................................................. 57

a. TiO-PAN Titanium Dioxide Composite …………......................................................... 58

b. NiFC-PAN Nickel Ferrocyanide Composite .................................................................59

c. MgO-PAN Magnesium Oxide Composite ……….............................................................6 60

d. MnO-PAN Manganese Dioxide Composite ……..........................................................6 60

e. NaY-PAN Synthetic Zeolite Composite ......................................................................6 62

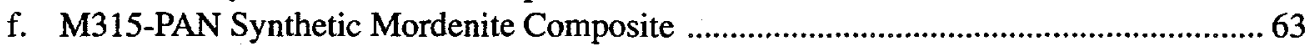

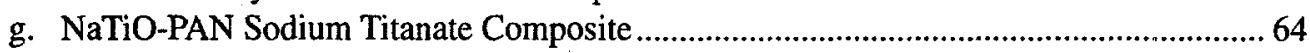

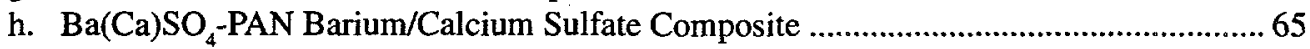

i. AMP-PAN Ammonium Molybdophosphate Composite ……………..............................6 66

4. Phenolsulfonic-Formaldehyde (PSF) Composite Absorbers ...................................................67

a. TiFC-PSF Titanium Hexacyanoferrate Composite ………................................................... 67

b. CoFC-PSF Cobalt Hexacyanoferrate Composite ………...................................................6 68

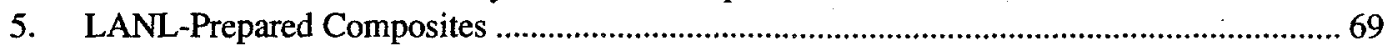

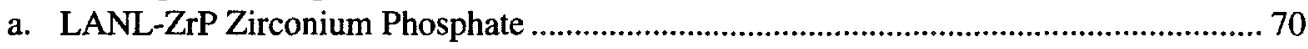

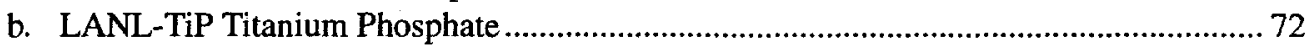

c. LANL-TiO Titanium Dioxide ................................................................................. 74

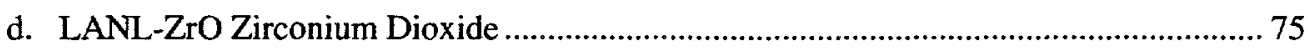

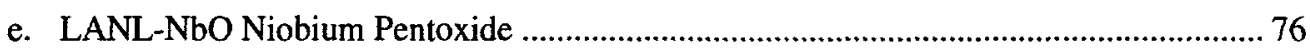

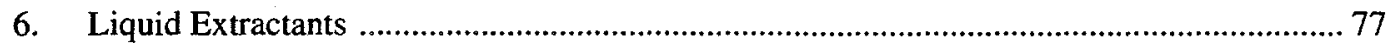

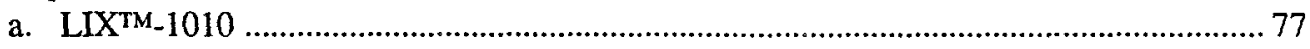

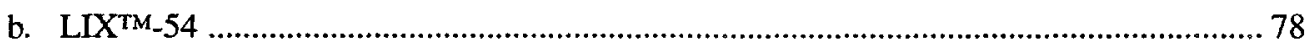

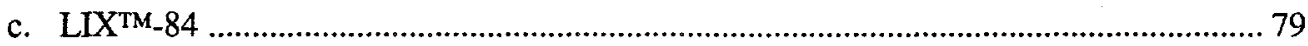

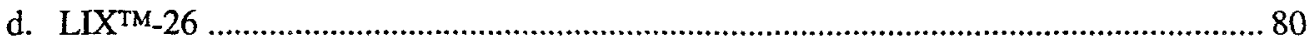

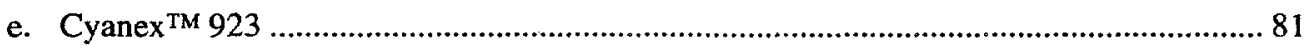

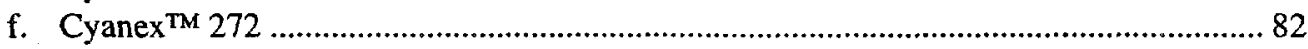

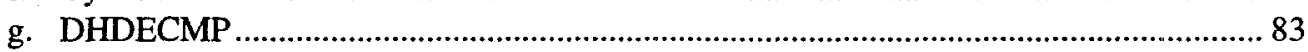

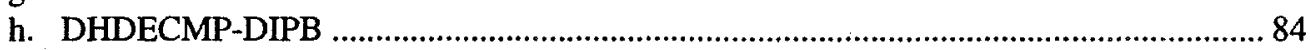

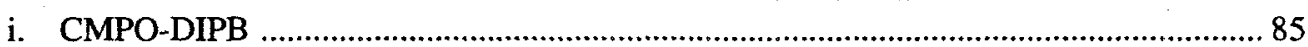

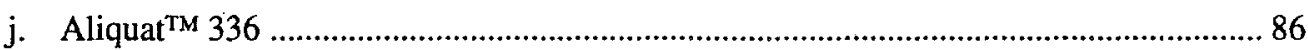

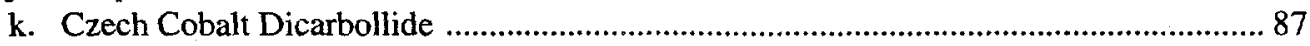

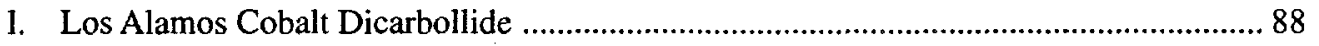

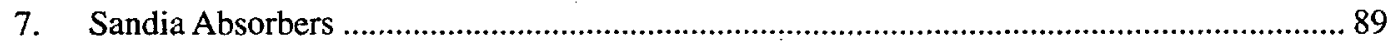

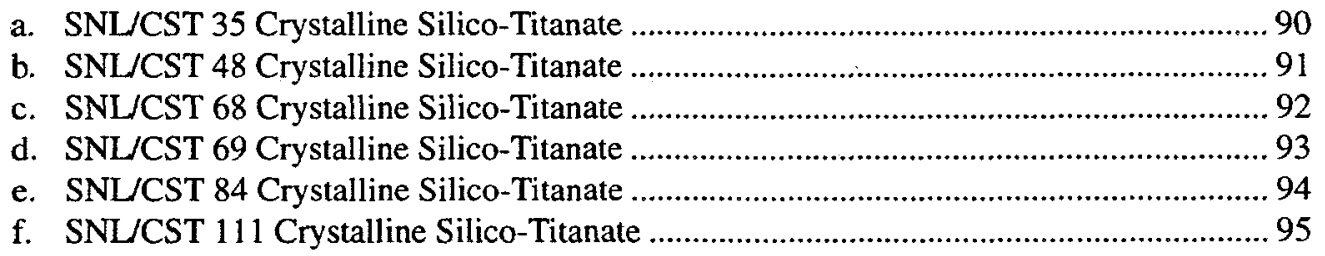




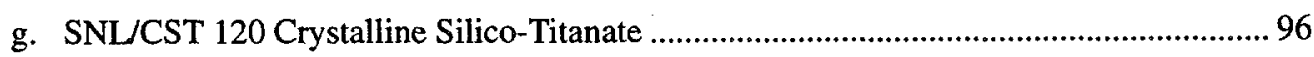

h. SNL/CST 141 Crystalline Silico-Titanate ....................................................................... 97

i. SNL/CST 149 Crystalline Silico-Titanate ........................................................................ 98

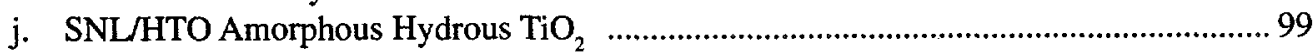

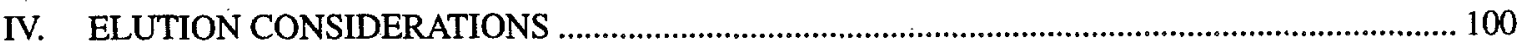

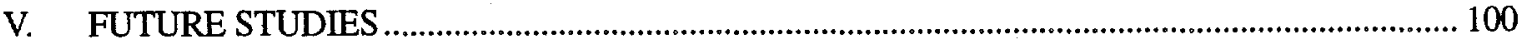

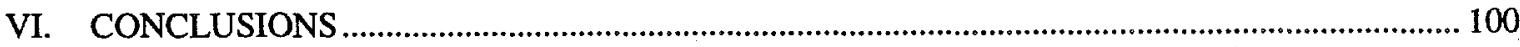

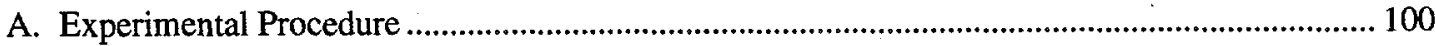

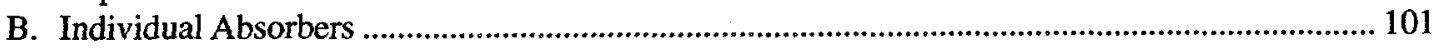

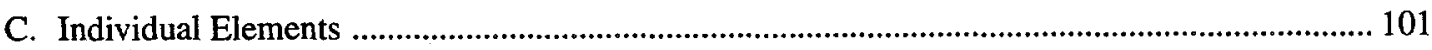

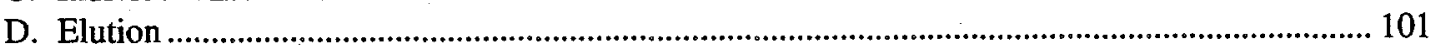

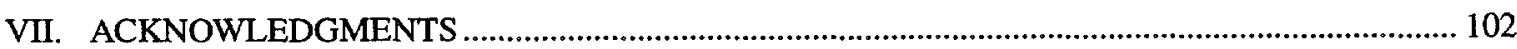

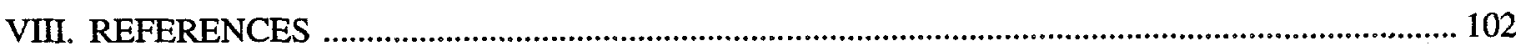

\section{LIST OF TABLES}

Table 1. Number of Absorbers Capable of Sorbing Each of 14 Elements

from Three Simulant Solutions ..................................................................................................... 2

Table 2. Compositions of Hanford Tank 102-SY Simulant Solutions ......................................................... 5

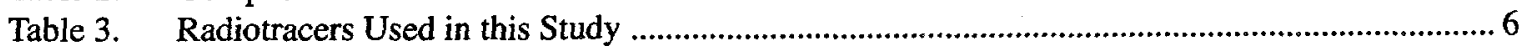

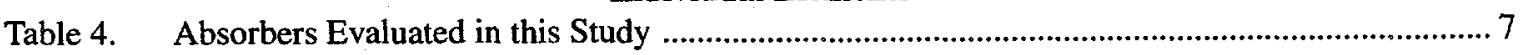

Table 5. Cerium Distribution Data ............................................................................................. 12

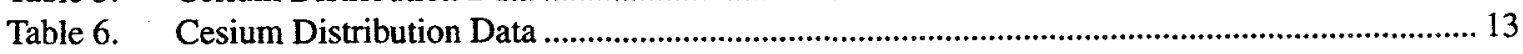

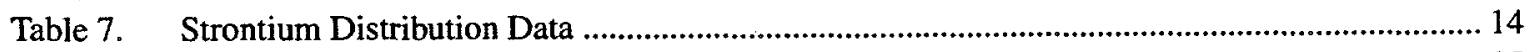

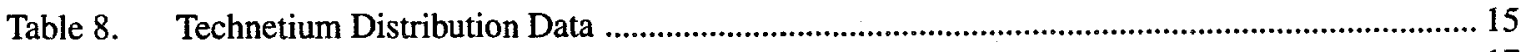

Table 9. Yttrium Distribution Data ............................................................................................ 17

Table 10. Chromium Distribution Data ............................................................................................. 18

Table 11. Cobalt Distribution Data ….............................................................................................. 19

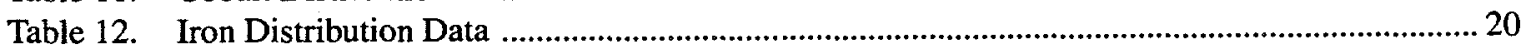

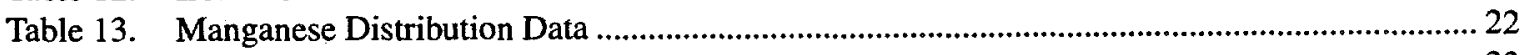

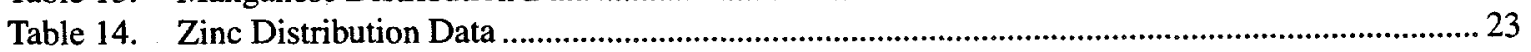

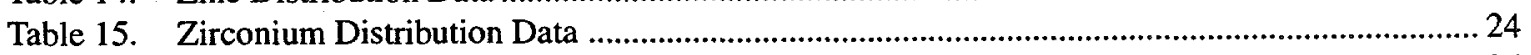

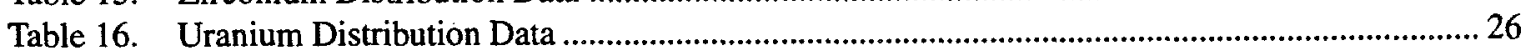

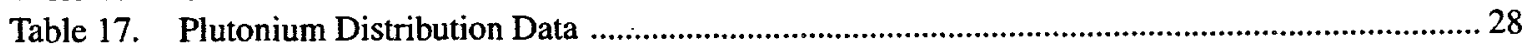

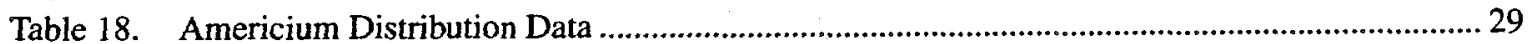

Commercially Available Absorbers

\section{Individual Absorbers}

Table 19. Amberlyst ${ }^{\mathrm{TM}} 15$ Cation Exchange Resin: Distribution of 14 Elements

from Three Simulant Solutions for Hanford Tank 102-SY

Table 20. Amberlyst ${ }^{\mathrm{TM}} \mathrm{XN}-1010$ Cation Exchange Resin: Distribution of 14 Elements from Three Simulant Solutions for Hanford Tank 102-SY

Table 21. Duolite ${ }^{\mathrm{TM}} \mathrm{C}-467$ Cation Exchange Resin: Distribution of 14 Elements from Three Simulant Solutions for Hanford Tank 102-SY

Table 22. Diphonix ${ }^{\mathrm{TM}}$ Cation Exchange Resin: Distribution of 14 Elements from Three Simulant Solutions for Hanford Tank 102-SY ......................................................... 34

Table 23. Ionsiv'M TIE-96: Distribution of 14 Elements from Three Simulant Solutions for Hanford Tank 102-SY 
Table 24. Ionsiv TM TIE-96 (Modified): Distribution of 14 Elements

from Three Simulant Solutions for Hanford Tank 102-SY

Table 25. Duolite ${ }^{\mathrm{TM}}$ CS-100 Cation Exchange Resin: Distribution of 14 Elements

from Three Simulant Solutions for Hanford Tank 102-SY

Table 26. Tannin: Distribution of 14 Elements from Three

Simulant Solutions for Hanford Tank 102-SY

Table 27. Sr-Spec TM Resin: Distribution of 14 Elements from Three

Simulant Solutions for Hanford Tank 102-SY

Table 28. TRU-Spec ${ }^{\text {TM }}$ Resin: Distribution of 14 Elements from Three

Simulant Solutions for Hanford Tank 102-SY

Table 29. Durasil ${ }^{\text {TM }} 190$ Resin: Distribution of 14 Elements from Three

Simulant Solutions for Hanford Tank 102-SY

Table 30. Durasil ${ }^{\mathrm{TM}} 230$ Resin: Distribution of 14 Elements from Three

Simulant Solutions for Hanford Tank 102-SY

Table 31. Bone Char: Distribution of 14 Elements from Three

Simulant Solutions for Hanford Tank 102-SY

Table 32. Reillex ${ }^{\mathrm{TM}} \mathrm{HPQ}$ Anion Exchange Resin: Distribution of 14 Elements

from Three Simulant Solutions for Hanford Tank 102-SY

Table 33. Ionac ${ }^{\text {TM }}$ SR-3 Anion Exchange Resin: Distribution of 14 Elements

from Three Simulant Solutions for Hanford Tank 102-SY

Table 34. Purolite ${ }^{\mathrm{TM}}$ A-520-E Anion Exchange Resin: Distribution of 14 Elements from Three Simulant Solutions for Hanford Tank 102-SY

Table 35. Ionac ${ }^{\text {TM }}$ SR-6 Anion Exchange Resin: Distribution of 14 Elements

from Three Simulant Solutions for Hanford Tank 102-SY

Table 36. SRS Resorcinol/Formaldehyde Resin: Distribution of 14 Elements

from Three Simulant Solutions for Hanford Tank 102-SY

Resins

Experimental Absorbers

Table 37. Sybron (Et) N Anion Exchange Resin: Distribution of 14 Elements

from Three Simulant Solutions for Hanford Tank 102-SY 50

Table 38. Sybron ( $\mathrm{Pr})_{3} \mathrm{~N}$ Anion Exchange Resin: Distribution of 14 Elements from Three Simulant Solutions for Hanford Tank 102-SY

Table 39. JSK-1 Anion Exchange Resin: Distribution of 14 Elements from Two Simulant Solutions for Hanford Tank 102-SY

Table 40. JSK-2 Anion Exchange Resin: Distribution of 14 Elements

from Two Simulant Solutions for Hanford Tank 102-SY

Table 41. JSK-3 Anion Exchange Resin: Distribution of 14 Elements

from Two Simulant Solutions for Hanford Tank 102-SY

Table 42. Crypt-DER Polymer Resin: Distribution of 14 Elements

from Three Simulant Solutions for Hanford Tank 102-SY

Table 43. PK-01-244 Resin: Distribution of 14 Elements from

Three Simulant Solutions for Hanford Tank 102-SY

PAN Composite Absorbers

Table 44. TiO-PAN Composite: Distribution of 14 Elements from

Three Simulant Solutions for Hanford Tank 102-SY

Table 45. NiFC-PAN Composite: Distribution of 14 Elements from

Three Simulant Solutions for Hanford Tank 102-SY

Table 46. MgO-PAN Composite: Distribution of 14 Elements from

Two Simulant Solutions for Hanford Tank 102-SY

Table 47. MnO-PAN Composite: Distribution of 14 Elements from

Three Simulant Solutions for Hanford Tank 102-SY

Table 48. NaY-PAN Composite: Distribution of 14 Elements from

Three Simulant Solutions for Hanford Tank 102-SY 
Table 49. M315-PAN Composite: Distribution of 14 Elements from Three Simulant Solutions for Hanford Tank 102-SY

Table 50. NaTiO-PAN Composite: Distribution of 14 Elements from Three Simulant Solutions for Hanford Tank 102-SY

Table 51. $\mathrm{Ba}(\mathrm{Ca}) \mathrm{SO}_{4}-\mathrm{PAN}$ Composite: Distribution of 14 Elements from Three Simulant Solutions for Hanford Tank 102-SY

Table 52. AMP-PAN Composite: Distribution of 14 Elements from Three Simulant Solutions for Hanford Tank 102-SY

PSF Composite Absorbers

Table 53. TiFC-PSF Composite: Distribution of 14 Elements from

Three Simulant Solutions for Hanford Tank 102-SY

Table 54. CoFC-PSF Composite: Distribution of 14 Elements from

Three Simulant Solutions for Hanford Tank 102-SY

\section{Los Alamos-Prepared Composites}

Table 55. LANL-ZrP Composite: Distribution of 14 Elements from

Three Simulant Solutions for Hanford Tank 102-SY

Table 56. LANL-TiP Composite: Distribution of 14 Elements from

Three Simulant Solutions for Hanford Tank 102-SY.

Table 57. LANL-TiO Composite: Distribution of 14 Elements from

Three Simulant Solutions for Hanford Tank 102-SY

Table 58. LANL-ZrO Composite: Distribution of 14 Elements from Three Simulant Solutions for Hanford Tank 102-SY

Table 59. LANL-NbO Composite: Distribution of 14 Elements from Three Simulant Solutions for Hanford Tank 102-SY

\section{Extractants}

Table 60. LIXTM-1010: Distribution of 14 Elements from

Three Simulant Solutions for Hanford Tank 102-SY

Table 61. LIX ${ }^{\mathrm{TM}}$-54: Distribution of 14 Elements from

Three Simulant Solutions for Hanford Tank 102-SY ............................................................ 78

Table 62. LIX ${ }^{\mathrm{TM}}-84$ : Distribution of 14 Elements from

Three Simulant Solutions for Hanford Tank 102-SY

Table 63. LIX ${ }^{\mathrm{TM}}-26$ : Distribution of 14 Elements from

Three Simulant Solutions for Hanford Tank 102-SY

Table 64. Cyanex ${ }^{\mathrm{TM}}$ 923: Distribution of 14 Elements from

Three Simulant Solutions for Hanford Tank 102-SY

Table 65. Cyanex ${ }^{\mathrm{TM}}$ 272: Distribution of 14 Elements from

Three Simulant Solutions for Hanford Tank 102-SY

Table 66. DHDECMP: Distribution of 14 Elements from

Three Simulant Solutions for Hanford Tank 102-SY

Table 67. DHDECMP-DIPB: Distribution of 14 Elements from

Three Simulant Solutions for Hanford HLW 102-SY

Table 68. CMPO-DIPB: Distribution of 14 Elements from

Three Simulant Solutions for Hanford Tank 102-SY

Table 69. Aliquat ${ }^{\mathrm{TM}}$ 336: Distribution of 14 Elements from

Three Simulant Solutions for Hanford Tank 102-SY

Table 70. Czech Cobalt Dicarbollide: Distribution of 14 Elements

from Two Simulant Solutions for Hanford Tank 102-SY

Table 71. Los Alamos Cobalt Dicarbollide: Distribution of 14 Elements from Two Simulant Solutions for Hanford Tank 102-SY

Inorganics

Table 72. SNL/CST 35: Distribution of 14 Elements from Three Simulant Solutions for Hanford Tank 102-SY .90

Table 73. SNL/CST 48: Distribution of 14 Elements from Two Simulant Solutions for Hanford Tank 102-SY 
Table 74. SNL/CST 68: Distribution of 14 Elements from

Two Simulant Solutions for Hanford Tank 102-SY

Table 75. SNL/CST 69: Distribution of 14 Elements from

Three Simulant Solutions for Hanford Tank 102-SY

Table 76. SNL/CST 84: Distribution of 14 Elements from

Two Simulant Solutions for Hanford Tank 102-SY

Table 77. SNL/CST 111: Distribution of 14 Elements from

Three Simulant Solutions for Hanford Tank 102-SY

Table 78. SNL/CST 120: Distribution of 14 Elements from Alkaline Supernate Simulant Solution for Hanford Tank 102-SY

Table 79. SNL/CST 141: Distribution of 14 Elements from Alkaline Supernate Simulant Solution for Hanford Tank 102-SY.

Table 80. SNL/CST 149: Distribution of 14 Elements from Alkaline Supernate Simulant Solution for Hanford Tank 102-SY 98

Table 81. SNL/HTO: Distribution of 14 Elements from

Two Simulant Solutions for Hanford Tank 102-SY 99

\section{LIST OF FIGURES}

Fig. 1. Hypodermic syringe, with porous Kynar filter in tip, as used for solution/absorber contacts. An uninstalled Kynar filter is shown below the syringe 9

Fig. 2. Tube rotator used to mix the solution/absorber combinations 9

\section{TRADEMARKS}

Aliquat is a registered trademark of the Henkel Corporation, Tucson, AZ, Tel. 602-622-8891.

Amberlyst is a registered trademark of Rohm \& Haas, Philadelphia, PA, Tel. 215-592-3000.

Cyanex is a registered trademark of American Cyanamid Company, Wayne, NJ, Tel. 800-438-5615.

Duolite is a registered trademark of Rohm \& Haas, Philadelphia, PA, Tel. 215-592-3000.

Durasil is a registered trademark of the Duratek Corporation, Columbia, MD, Tel. 410-312-5100.

EXCEL is a registered trademark of Microsoft Corporation, Redmond, WA, Tel. 800-426-9400.

Ionac is a registered trademark of Sybron Chemicals, Birmingham, NJ, Tel. 609-893-1100.

Ionsiv is a registered trademark of UOP Molecular Sieves, Mt. Laurel, NJ, Tel. 609-727-9400.

LIX is a registered trademark of the Henkel Corporation, Tucson, AX, Tel. 602-622-8891.

Purolite is a registered trademark of the Purolite Company, Bala Cynwyd, PA, Tel. 215-668-9090.

Sr-Spec is a registered trademark of EIChroM Industries, Darien, IL, Tel. 800-424-9300.

TRU-Spec is a registered trademark of EIChroM Industries, Darien, IL, Tel. 800-424-9300. 


\title{
DISTRIBUTIONS OF 14 ELEMENTS ON 63 ABSORBERS \\ FROM THREE SIMULANT SOLUTIONS (ACID-DISSOLVED SLUDGE, ACIDIFIED \\ SUPERNATE, AND ALKALINE SUPERNATE) FOR HANFORD HLW TANK 102-SY
}

by

\author{
S. Fredric Marsh, Zita V. Svitra, and Scott M. Bowen
}

\begin{abstract}
As part of the Hanford Tank Waste Remediation System program at Los Alamos, we evaluated 63 commercially available or experimental absorber materials for their ability to remove hazardous components from high-level waste (HLW). These absorbers included cation and anion exchange resins, inorganic exchangers, composite absorbers, and a series of liquid extractants sorbed on porous support-beads. We tested these absorbers with three solutions prepared to simulate acid-dissolved sludge (pH 0.6), acidified supernate ( $\mathrm{pH}$ 3.5), and alkaline supernate ( $\mathrm{pH}$ 13.9) from underground storage tank 102-SY at the Hanford Reservation near Richland, Washington. To these simulants we added the appropriate radionuclides and used gamma spectrometry to measure fission products ( $\mathrm{Ce}, \mathrm{Cs}, \mathrm{Sr}, \mathrm{Tc}$, and $\mathrm{Y}$ ), actinides (U, $\mathrm{Pu}$, and $\mathrm{Am}$ ), and matrix elements ( $\mathrm{Cr}, \mathrm{Co}, \mathrm{Fe}, \mathrm{Mn}, \mathrm{Zn}$, and $\mathrm{Zr}$ ). For each of more than 2500 element/absorber/solution combinations, we measured distribution coefficients for dynamic contact periods of $30 \mathrm{~min}, 2 \mathrm{~h}$, and $6 \mathrm{~h}$ to obtain information about sorption kinetics. Because we measured the sorption of many different elements, the tabulated results indicate those elements most likely to interfere with the sorption of elements of greater interest. On the basis of nearly 7500 measured distribution coefficients, we determined that many of these absorbers appear suitable for processing HLW. This study supersedes the previous version of LA-12654, in which results attributed to a solution identified as an alkaline supernate simulant were misleading because that solution contained insufficient hydroxide.
\end{abstract}

\section{EXECUTIVE SUMMARY}

Successful remediation of the large quantities of hazardous waste stored in underground tanks at the Hanford Reservation near Richland, Washington, requires the identification of reliable partitioning agents and the development of suitable technologies. To address this need, we measured the sorption of 14 elements onto more than 60 different absorbers from simulants that represent acid-dissolved sludge, acidified supernate, and alkaline supernate solutions from Hanford Tank 102-SY.

Our results indicate that many existing partitioning agents may be suitable for HLW tank remediation. The identification of reliable partitioning agents could allow the processing of HLW in Hanford tanks to begin and be completed sooner, and at a lower cost, than would otherwise be possible. The findings of our study could have a major beneficial impact on decommissioning and environmental remediation efforts at Hanford and elsewhere within the U.S. Department of Energy (DOE) complex.

This screening study, intended to identify the most promising absorbers and extractants, included mainly absorbers that either are already commercially available or could be produced in commercial quantities at acceptable cost and within a reasonable time. Distribution coefficients for each element/absorber/solution combination were measured for dynamic contact periods of 30 $\min , 2 \mathrm{~h}$, and $6 \mathrm{~h}$ to provide information about the sorption kinetics of each system. 
The observed behavior of the absorber/element combination often was significantly different from the published behavior of the same combination with relatively clean solutions. We attribute such differences to the effects of other cations that compete for absorber sites, as well as to the competition from anions that form soluble complexes with metal ions that would otherwise be sorbed. Such matrix-dependent differences in absorber performance demonstrate the value of using realistic simulant solutions for such measurements.

We also found numerous inexpensive commercial materials that outperform specialty products costing much more.

Table 1 summarizes the number of absorbers identified as effective in each of six distribution coefficient (Kd) ranges.

Table 1. Number of Absorbers Capable of Sorbing Each of 14 Elements from Three Simulant Solutions

\begin{tabular}{|c|c|c|c|c|c|c|c|}
\hline \multirow[b]{2}{*}{ Solution } & \multirow[b]{2}{*}{ Element } & \multicolumn{6}{|c|}{ Kd Values } \\
\hline & & $>1000$ & $300-1000$ & $100-300$ & $20-100$ & $10-20$ & 5-10 \\
\hline Acid- & $\mathrm{Ce}$ & 0 & 0 & 0 & 5 & 1 & 1 \\
\hline Dissolved & Cs & 7 & 0 & 0 & 11 & 1 & 4 \\
\hline \multirow[t]{12}{*}{ Sludge } & $\mathrm{Sr}$ & 0 & 0 & 0 & 0 & 0 & 0 \\
\hline & $\mathrm{Tc}$ & 0 & 0 & 1 & 8 & 2 & 5 \\
\hline & $\mathrm{Y}$ & 0 & 0 & 0 & 0 & 0 & 0 \\
\hline & $\mathrm{Cr}$ & 0 & 0 & 0 & 0 & 0 & 0 \\
\hline & Co & 0 & 0 & 0 & 0 & 1 & 0 \\
\hline & $\mathrm{Fe}$ & 0 & 0 & 0 & 0 & 0 & 0 \\
\hline & $\mathrm{Mn}$ & 0 & 0 & 0 & 0 & 0 & 0 \\
\hline & $\mathrm{Zn}$ & 0 & 0 & 0 & 0 & 0 & 0 \\
\hline & $\mathrm{Zr}$ & 0 & 1 & 3 & 10 & 4 & 3 \\
\hline & $\mathrm{U}$ & 0 & 0 & 0 & 6 & 8 & 5 \\
\hline & $\mathrm{Pu}$ & 2 & 5 & 6 & 2 & 8 & 2 \\
\hline & Am & 0 & 0 & 0 & 0 & 0 & 6 \\
\hline Acidified & $\mathrm{Ce}$ & 1 & 3 & 3 & 10 & 3 & 6 \\
\hline \multirow[t]{13}{*}{ Supernate } & Cs & 8 & 1 & 5 & 2 & 4 & 2 \\
\hline & $\mathrm{Sr}$ & 0 & 0 & 0 & 0 & 3 & 4 \\
\hline & Tc & 0 & 7 & 7 & 9 & 3 & 1 \\
\hline & $\mathrm{Y}$ & 1 & 0 & 1 & 9 & 0 & 5 \\
\hline & $\mathrm{Cr}$ & 0 & 0 & 0 & 2 & 2 & 5 \\
\hline & Co & 0 & 0 & 0 & 1 & 3 & 4 \\
\hline & $\mathrm{Fe}$ & 0 & 0 & 0 & 0 & 0 & 3 \\
\hline & $\mathrm{Mn}$ & 0 & 0 & 0 & 0 & 1 & 5 \\
\hline & $\mathrm{Zn}$ & 0 & 0 & 0 & 5 & 3 & 2 \\
\hline & $\mathrm{Zr}$ & 0 & 0 & 0 & 1 & 4 & 3 \\
\hline & $\mathrm{U}$ & 0 & 1 & 4 & 15 & 8 & 8 \\
\hline & $\mathrm{Pu}$ & 0 & 0 & 0 & 0 & 0 & 1 \\
\hline & $\mathrm{Am}$ & 0 & 3 & 2 & 13 & 1 & 5 \\
\hline Alkaline & $\mathrm{Ce}$ & 1 & 4 & 4 & 5 & 3 & 15 \\
\hline \multirow[t]{12}{*}{ Supernate } & Cs & 5 & 3 & 6 & 2 & 2 & 2 \\
\hline & $\mathrm{Sr}$ & 12 & 6 & 4 & 7 & 8 & 7 \\
\hline & Tc & 0 & 6 & 2 & 4 & 1 & 3 \\
\hline & $Y$ & 0 & 1 & 3 & 20 & 7 & 6 \\
\hline & $\mathrm{Cr}$ & 0 & 0 & 0 & 2 & 0 & 0 \\
\hline & Co & 0 & 0 & 0 & 1 & 5 & 1 \\
\hline & $\mathrm{Fe}$ & 6 & 2 & 10 & 10 & 3 & 3 \\
\hline & $\mathrm{Mn}$ & 3 & 5 & 9 & 5 & 6 & 5 \\
\hline & $\mathrm{Zn}$ & 8 & 5 & 5 & 3 & 2 & 2 \\
\hline & $\mathrm{Zr}$ & 3 & 13 & 7 & 6 & 2 & 0 \\
\hline & $\mathrm{U}$ & 2 & 1 & 5 & 5 & 4 & 3 \\
\hline & $\mathrm{Am}$ & 1 & 5 & 1 & 4 & 3 & 1 \\
\hline
\end{tabular}


From acid-dissolved sludge solution, cerium, cesium, technetium, zirconium, uranium, and plutonium show good to excellent sorption, whereas strontium, yttrium, chromium, cobalt, iron, manganese, zinc, and americium show poor sorption.

From acidified supernate solution, cerium, cesium, technetium, yttrium, zinc, uranium, and americium show good to excellent sorption, whereas strontium, chromium, cobalt, iron, manganese, zirconium, and plutonium show poor to marginal sorption.

From alkaline supernate solution, cerium, cesium, strontium, yttrium, iron, manganese, zinc, zirconium, uranium, and americium show good to excellent sorption, whereas chromium and cobalt show low to moderate sorption.

Those absorbers identified as acceptable on the basis of these initial tests are being evaluated to determine their effectiveness in sorbing many elements from solutions that simulate the contents of other underground storage tanks at Hanford. Moreover, we plan to begin studies using actual radioactive waste solutions as soon as we can obtain them. We also suggest an accelerated effort to identify or develop partitioning agents for specific needs, such as sorbing strontium from acidic solution, for which we identified no satisfactory absorbers.

This report supersedes, corrects, and expands the previously published Los Alamos National Laboratory report LA-12654, which included distribution data from an acidified supernate solution incorrectly identified as an alkaline supernate solution. Readers should destroy any copies of LA-12654 in their possession.

\section{INTRODUCTION}

The Hanford Reservation near Richland, Washington, incorporates 177 underground tanks that store more than 65 million gallons of radioactive waste containing some 165 million curies. These high-level wastes are a byproduct of the production of nuclear materials for national defense needs during the past half-century. Because Hanford operating contractors used numerous chemical processes during this period, many different reagents and waste streams were generated and combined in underground tanks, often with insufficient concern about the compatibility of the various components. The resulting wastes consist of complex and sometimes unstable mixtures of sludges, saltcakes, slurries, and supernates. Adding significantly to the stored-waste problem is the fact that 67 of these tanks are known, or are presumed, to have leaked.

The U.S. Department of Energy (DOE) is committed to the remediation of hazardous wastes stored at Hanford and has directed Los Alamos National Laboratory (LANL) to support the Hanford Tank Waste
Remediation System (TWRS) mission, which is to store, treat, and dispose of all tank waste in a safe, costeffective, and environmentally sound manner. An essential prerequisite for achieving this goal is the identification of suitable partitioning agents and technologies.

Ion exchange is a partitioning technology that has been extensively used in the nuclear industry. Anion exchange has been used for many decades to recover plutonium $^{1}$ and neptunium ${ }^{2}$ from a wide variety of impure nuclear materials. Although cation exchange resins also have been used in the nuclear industry, ${ }^{3}$ they generally are considered to be less selective than anion exchange resins.

Although most of the absorbers we tested are cation exchangers, we also included anion exchange resins, inorganic exchangers, composite resins, and a series of liquid ion exchangers (sorbed on porous carbon beads). Our intent was to obtain more comprehensive data as well as to supply convenient reference points for other studies. We also included some experimental absorbers to provide guidance for future research activities.

Some of the absorbers included in our study have been reported by others to offer high selectivity for specific ions; however, few of these absorbers have been evaluated with media approaching the complexity of the HLW solutions stored at Hanford. Nor have any other investigators, to our knowledge, measured the distribution of as many elements (14) onto so many different absorber materials (60) under identical test conditions. Moreover, because we measured the sorption of so many different elements, our study provides selectivity information that identifies which unwanted elements are most likely to interfere by competing for absorber sites.

Our objective was to evaluate numerous potentially useful absorber materials, including many not previously studied, for their ability to recover selected elements from realistic simulants of acid-dissolved sludge, acidified supernate, and alkaline supernate solutions for Hanford Tank 102-SY. Because no simulant can accurately represent the contents of any HLW storage tank, the most promising absorbers eventually must be tested with actual waste. However, because actual waste samples are expensive and difficult to obtain, we advocate preliminary screening of promising candidate $a b-$ sorbers with realistic simulant solutions before testing with actual tank waste solutions.

Our study initially was intended to support the Los Alamos flow sheet development program for Hanford Tank 102-SY; ${ }^{4}$ however, many of our experimental findings apply to other flow sheet proposals, such as the socalled "Clean Option." Although we include comments and observations about our experimental data, we intentionally do not recommend specific absorbers. Our purpose is to identify options from which scientists can 
develop reliable process flow sheets. The preferred partitioning options will depend on the objectives of the specific flow sheet. Decisions about whether the targeted elements should be recovered individually or grouped for subsequent disposal are properly left to the flow sheet designers. No matter which flow sheet is adopted, however, our experimental data will enable HLW tank processing to begin and be completed sooner and at a lower cost than would otherwise be possible.

\section{EXPERIMENTAL PARAMETERS}

\section{A. Simulant Solutions}

The compositions of the simulant solutions for Hanford HLW Tank 102-SY were based on consultation with and recommendations from Westinghouse Hanford Company (WHC) and Battelle Pacific Northwest Laboratories (PNL) personnel. ${ }^{6}$ The sludge composition was based on the assumption that soluble components had been leached out during thorough mixing with the supernate solutions. Our assumptions about tank contents were checked against composition data obtained by reconstruction of the tank contents from all available records for transfers into and out of each tank during its entire operating history. ${ }^{7}$
The sludge components that are expected to dissolve during sludge-washing operations ${ }^{8}$ were subtracted from our best estimate of the initial sludge composition. We dissolved those components assumed to remain in the sludge in a minimum quantity of nitric acid. The simulated acid-dissolved sludge was filtered to remove insoluble residue.

The alkaline supernate and acidified supernate solutions were similar in their initial composition, but the alkaline supernate solution contained substantially more hydroxide and generated much more precipitate. To ensure that the alkaline supernate solution was truly saturated with relatively insoluble salts, we diluted the initial liter of solution/solids with another three liters of water and heated this diluted solution in a stainless steel container while stirring continuously until the volume was reduced to the initial one liter. Bulk solids were separated by centrifugation; any remaining suspended solids were removed by passing the alkaline supernate solution through a $0.45-\mu \mathrm{m}$ filter.

The compositions of the simulated acid-dissolved sludge, acidified supernate, and alkaline supernate solutions for Hanford Tank 102-SY are presented in Table 2. 
Table 2. Compositions of Hanford Tank 102-SY Simulant Solutions

\begin{tabular}{|c|c|c|c|}
\hline & $\begin{array}{l}\text { Acid-Dissolved } \\
\text { Sludge }\end{array}$ & $\begin{array}{c}\text { Acidified } \\
\text { Supernate }\end{array}$ & $\begin{array}{c}\text { Alkaline } \\
\text { Supernate }\end{array}$ \\
\hline \multicolumn{4}{|l|}{ Cations $^{a}$} \\
\hline $\mathrm{Na}$ & $0.65 \mathrm{M}$ & $1.40 \mathrm{M}$ & $2.2 \mathrm{M}$ \\
\hline $\mathrm{Mg}$ & $0.060 \mathrm{M}$ & $0.032 \mathrm{M}$ & none \\
\hline $\mathrm{Al}$ & $0.43 \mathrm{M}$ & $0.44 \mathrm{M}$ & $0.16 \mathrm{M}$ \\
\hline $\mathrm{Si}$ & $0.125 \mathrm{M}$ & $0.29 \mathrm{M}$ & $0.0024 \mathrm{M}$ \\
\hline $\mathrm{Ca}$ & $0.044 \mathrm{M}$ & $0.028 \mathrm{M}$ & $0.0069 \mathrm{M}$ \\
\hline $\mathrm{Cr}(\mathrm{III})$ & $0.33 \mathrm{M}$ & $0.072 \mathrm{M}$ & none \\
\hline $\mathrm{Cr}(\mathrm{VI})$ & none & $0.007 \mathrm{M}$ & $0.0051 \mathrm{M}$ \\
\hline $\mathrm{Mn}$ & $0.143 \mathrm{M}$ & $0.043 \mathrm{M}$ & none \\
\hline $\mathrm{Fe}$ & $0.36 \mathrm{M}$ & $0.101 \mathrm{M}$ & $0.0061 \mathrm{M}$ \\
\hline $\mathrm{Ni}$ & $0.010 \mathrm{M}$ & $\mathrm{BDL}^{\mathrm{c}}$ & $\mathrm{BDL}$ \\
\hline $\mathrm{Cu}$ & $0.016 \mathrm{M}$ & $\mathrm{BDL}$ & $\mathrm{BDL}$ \\
\hline $\mathrm{Se}$ & $0.027 \mathrm{M}$ & $\mathrm{BDL}$ & BDL \\
\hline $\mathrm{Sr}$ & $0.0009 \mathrm{M}$ & BDL & BDL \\
\hline $\mathrm{Pb}$ & $0.012 \mathrm{M}$ & $\mathrm{BDL}$ & BDL \\
\hline Th & $0.027 \mathrm{M}$ & $\mathrm{BDL}$ & BDL \\
\hline $\mathbf{U}$ & $0.023 \mathrm{M}$ & BDL & BDL \\
\hline${ }^{239} \mathrm{Pu}$ & $3 \mathrm{~g} / \mathrm{L}$ & - & $\mathrm{BDL}$ \\
\hline${ }^{238} \mathrm{Pu}$ & $60 \mathrm{mg} / \mathrm{L}$ & $60 \mathrm{mg} / \mathrm{L}$ & none \\
\hline \multicolumn{4}{|l|}{ Anions $^{b}$} \\
\hline $\mathrm{F}$ & $0.027 \mathrm{M}$ & 0.024 & $0.21 \mathrm{M}$ \\
\hline $\mathrm{Cl}$ & $0.006 \mathrm{M}$ & 0.021 & $0.102 \mathrm{M}$ \\
\hline $\mathrm{NO}_{3}$ & $5.24 \mathrm{M}$ & 1.95 & $1.31 \mathrm{M}$ \\
\hline $\mathrm{PO}_{4}^{3}$ & $0.006 \mathrm{M}$ & $<0.002$ & $0.061 \mathrm{M}$ \\
\hline $\mathrm{SO}_{4}^{4}$ & $0.009 \mathrm{M}$ & 0.015 & $0.022 \mathrm{M}$ \\
\hline$\overline{\mathrm{pH}}$ & 0.58 & 3.5 & 13.85 \\
\hline calculated $\mathrm{H}^{+}$ & $0.5 \mathrm{M}$ & - & - \\
\hline calculated $\mathrm{OH}^{-}$ & - & - & $0.7 \mathrm{M}$ \\
\hline \multicolumn{4}{|c|}{$\begin{array}{l}\text { aDetermined by inductively coupled plasma/mass spectrometry (ICP/MS). } \\
\text { bDetermined by ion chromatography (IC). } \\
\text { 'Below detection limit. }\end{array}$} \\
\hline
\end{tabular}




\section{B. Radiotracers}

Having selected gamma spectrometry as the technique for measuring the distribution of the selected elements, we obtained suitable radiotracers to be added to the simulant solutions. The selected tracers and the gamma energies measured are listed in Table 3.

Actinides were isolated and purified in the Los Alamos Plutonium Facility. We purchased ${ }^{51} \mathrm{Cr},{ }^{141} \mathrm{Ce}$, and ${ }^{60} \mathrm{Co}$ from DuPont New England Nuclear Products, Boston, MA. All other radioisotopes were obtained from the Medical Radioisotopes and Reactor Applications Group (CST-15) at Los Alamos National Laboratory.
To minimize interference among gamma rays with similar energies, we divided the 14 radiotracers into two easily resolved groups. One group consisted of ${ }^{141} \mathrm{Ce}$, ${ }^{60} \mathrm{Co},{ }^{51} \mathrm{Cr},{ }^{137} \mathrm{Cs},{ }^{85} \mathrm{Sr},{ }^{95 \mathrm{~m}} \mathrm{Tc},{ }^{88} \mathrm{Y}$, and ${ }^{88} \mathrm{Zr}$. The other group contained ${ }^{241} \mathrm{Am},{ }^{59} \mathrm{Fe},{ }^{54} \mathrm{Mn}$, either ${ }^{238} \mathrm{Pu}$ or ${ }^{239} \mathrm{Pu},{ }^{237} \mathrm{U}$, and ${ }^{65} \mathrm{Zn}$. Each group of tracers was added to a separate portion of each simulant solution. We then divided each simulant solution into two equal portions and added eight tracers to one portion and six tracers to the other portion of each simulant. (Plutonium was measured only in the two acidic solutions because its solubility was known to be very low in alkaline solution.)

\begin{tabular}{lccc}
\hline \hline \multicolumn{4}{l}{ Table 3. Radiotracers Used in this Study } \\
\hline & & $\begin{array}{c}\text { Gamma } \\
\text { Branching }(\%)^{\mathrm{a}}\end{array}$ & $\begin{array}{c}\text { Estimated } \\
\text { Concentration }^{\mathrm{b}}\end{array}$ \\
\hline Radiotracer & Gamma Energy (MeV) & 35.9 & $30 \mu \mathrm{g} / \mathrm{L}$ \\
${ }^{241} \mathrm{Am}$ & 0.0595 & $6.2 \times 10^{-3}$ & $3 \mathrm{~g} / \mathrm{L}$ \\
${ }^{239} \mathrm{Pu}$ & 0.1293 & $1.0 \times 10^{-3}$ & $60 \mathrm{mg} / \mathrm{L}$ \\
${ }^{238} \mathrm{Pu}$ & 0.153 & 48 & $50 \mathrm{pg} / \mathrm{L}$ \\
${ }^{141} \mathrm{Ce}$ & 0.145 & 100 & $2 \mathrm{pg} / \mathrm{L}$ \\
${ }^{95} \mathrm{Tc}$ & 0.204 & 23.3 & $20 \mathrm{mg} / \mathrm{L}$ \\
${ }^{237} \mathrm{U}$ & 0.208 & 10 & - \\
${ }^{51} \mathrm{Cr}$ & 0.320 & 97 & $50 \mu \mathrm{g} / \mathrm{L}$ \\
${ }^{88} \mathrm{Zr}$ & 0.394 & 100 & $3 \mu \mathrm{g} / \mathrm{L}$ \\
${ }^{85} \mathrm{Sr}$ & 0.514 & 85 & $6 \mu \mathrm{g} / \mathrm{L}$ \\
${ }^{137} \mathrm{Cs}$ & 0.662 & 100 & - \\
${ }^{54} \mathrm{Mn}$ & 0.835 & 92 & $50 \mu \mathrm{g} / \mathrm{L}$ \\
${ }^{88} \mathrm{Y}$ & 0.898 & 56 & - \\
${ }^{59} \mathrm{Fe}$ & 1.099 & 51 & $6 \mu \mathrm{g} / \mathrm{L}$ \\
${ }^{65} \mathrm{Zn}$ & 1.115 & 100 & $2 \mu \mathrm{g} / \mathrm{L}$ \\
${ }^{60} \mathrm{Co}$ & 1.173 & &
\end{tabular}

aGamma branching information is provided to indicate the assay sensitivity.

${ }^{\mathrm{b}}$ The elemental concentrations added with ${ }^{51} \mathrm{Cr},{ }^{54} \mathrm{Mn}$, and ${ }^{59} \mathrm{Fe}$ were insignificant when compared with the large quantities of these same elements in the simulant matrix (see Table 2). The elemental concentrations given for the other radiotracers are based on the best information available from suppliers; however, these values do not account for low levels of these elements, which could have been unintentionally added as impurities during simulant preparation.

${ }^{c}{ }^{238} \mathrm{Pu}$ was used at a concentration of $60 \mathrm{mg} / \mathrm{L}$ with all absorbers tested in acidified supernate solution and with a few selected absorbers in acid-dissolved sludge solution. For all other acid-dissolved sludge solutions, ${ }^{239} \mathrm{Pu}$ was used at $3 \mathrm{~g} / \mathrm{L}$ (a 50 -fold higher concentration than when ${ }^{238} \mathrm{Pu}$ was used). 


\section{Absorbers}

The absorbers evaluated in this study are listed in Table 4. Details about the supplier or preparation of the absorbers are provided in section III.B. These absorbers, which encompassed a wide range of particle sizes and porosities, were air-dried and used without further treatment except where otherwise noted.

Although the suppliers of the PAN composites, tannin, and Diphonix ${ }^{\mathrm{TM}}$ requested that their absorbers not be dried, we intentionally air-dried all absorbers before testing to put them all on a comparable basis. We also sought to avoid diluting the simulant solution by the addition of as-received wet absorbers; in the worst case the water in the as-received absorbers would have diluted the simulant by $23 \%$. Because our three simulant solutions were essentially saturated with various salts, large dilutions could significantly change the chemical properties of the simulant, thereby substantially affecting the performance of many absorbers. Nevertheless, we recognize that air-drying some of the absorbers also could adversely affect their performance, especially the sorption kinetics. We recommend that the Kd values we report for the PAN composites, tannin, and Diphonix ${ }^{\mathrm{TM}}$ be considered as minimum values that might have been higher had we not air-dried these absorbers.

Some absorbers were not compatible with all three simulant solutions, whereas others became available to us only after the study was under way. For these reasons, we did not measure all possible element/absorber/solution combinations.

Table 4. Absorbers Evaluated in this Study

\begin{tabular}{|c|c|}
\hline Absorber Type/Source & Tradename or Other Identification \\
\hline Commercial & 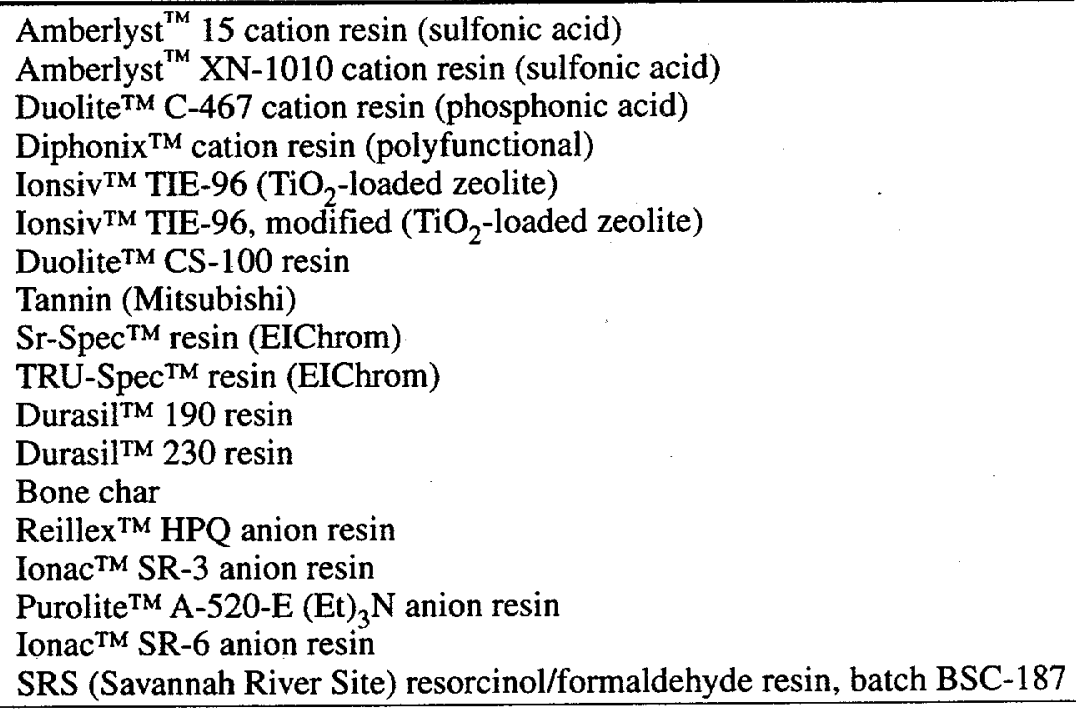 \\
\hline Experimental & $\begin{array}{l}\text { Sybron }^{\mathrm{TM}}(\mathrm{Et})_{3} \mathrm{~N} \text { anion resin } \\
\text { Sybron }^{\mathrm{TM}}(\mathrm{Pr})_{3} \mathrm{~N} \text { anion resin } \\
\text { JSK-1 anion resin } \\
\text { JSK-2 anion resin } \\
\text { JSK-3 anion resin } \\
\text { Crypt-DER polymer resin } \\
\text { PK-01-244 phosphinic acid resin }\end{array}$ \\
\hline
\end{tabular}

Composites

Sebesta, Czech Republic

(polyacrylonitrile matrix)
TiO-PAN titanium dioxide NiFC-PAN nickel ferrocyanide MgO-PAN magnesium oxide MnO-PAN manganese dioxide

NaY-PAN synthetic zeolite M315-PAN synthetic mordenite NaTiO-PAN sodium titanate $\mathrm{Ba}(\mathrm{Ca}) \mathrm{SO}_{4}-\mathrm{PAN}$ barium/calcium sulfate AMP-PAN ammonium molybdophosphate 
Table 4. Absorbers Evaluated in this Study ${ }^{\text {a }}$ (Cont.)

\begin{tabular}{ll}
\hline Absorber Type/Source & Tradename or Other Identification \\
\hline Narbutt, Poland & TiFC-PSF titanium hexacyanoferrate \\
(phenolsulfonic-formaldehyde matrix) & CoFC-PSF cobalt hexacyanoferrate \\
Los Alamos & LANL-ZrP zirconium phosphate \\
& LANL-TiP titanium phosphate \\
& LANL-TiO titanium dioxide \\
& LANL-ZrO zirconium dioxide \\
& LANL-NbO niobium pentoxide \\
\hline Extractant Beads & LIXTM-1010 (di-2-ethylhexylphosphoric acid) \\
Los Alamos & LIX'54 (a beta diketone) \\
& LIXT-84 (a ketoxime) \\
& LIX ${ }^{T M}-26$ (8-hydroxyquinoline) \\
& Cyanex 923 (trialkylphosphine oxide) \\
& Cyanex 272 (trimethylpentylphosphoric acid) \\
& DHDECMP \\
DHDECMP-diisopropylbenzene \\
CMPO-diisopropylbenzene \\
Aliquat 336 \\
Czech cobalt dicarbollide \\
Los Alamos-synthesized cobalt dicarbollide \\
\hline
\end{tabular}

\section{Inorganics}

Sandia National

Laboratories
SNL/CST 35 crystalline silico-titanate SNL/CST 48 crystalline silico-titanate SNL/CST 35 crystalline silico-titanate SNL/CST 68 crystalline silico-titanate SNL/CST 69 crystalline silico-titanate SNL/CST 84 crystalline silico-titanate SNL/CST 111 crystalline silico-titanate SNL/CST 120 crystalline silico-titanate SNL/CST 141 crystalline silico-titanate SNL/CST 149 crystalline silico-titanate SNL/HTO amorphous hydrous titanium oxide

Trademarks are identified on page $x$.

\section{Solution/Absorber Contacts}

In most cases, a $250-\mathrm{mg}$ portion of each air-dried absorber was contacted with a measured $6-\mathrm{mL}$ volume of each solution in a specially modified $20-\mathrm{mL}$ disposable hypodermic syringe. We modified these syringes by inserting cylindrical plugs cut from quarter-inch-thick porous Kynar, obtained from Porex Technologies; Fairburn, GA, into the tapered tips as filters, which permitted only liquid to pass through (Fig. 1).
For each set of experiments, we selected six syringes, each containing a different absorber. A measured volume of the selected solution was transferred by pipet into a plastic beaker and then drawn into a syringe through the Kynar filter. We then sealed the tip of each syringe with a solid Luer cap and placed the syringes on a 48-rpm tube rotator (Fig. 2) for dynamic contact periods of $30 \mathrm{~min}, 2 \mathrm{~h}$, and $6 \mathrm{~h}$. At the end of each contact period, approximately $25 \%$ of the total solution volume was expelled through the filtered tip of the syringe into a tared counting vial. We then weighed the dispensed solution and used that figure in the calculation of $\mathrm{Kd}$ values. 


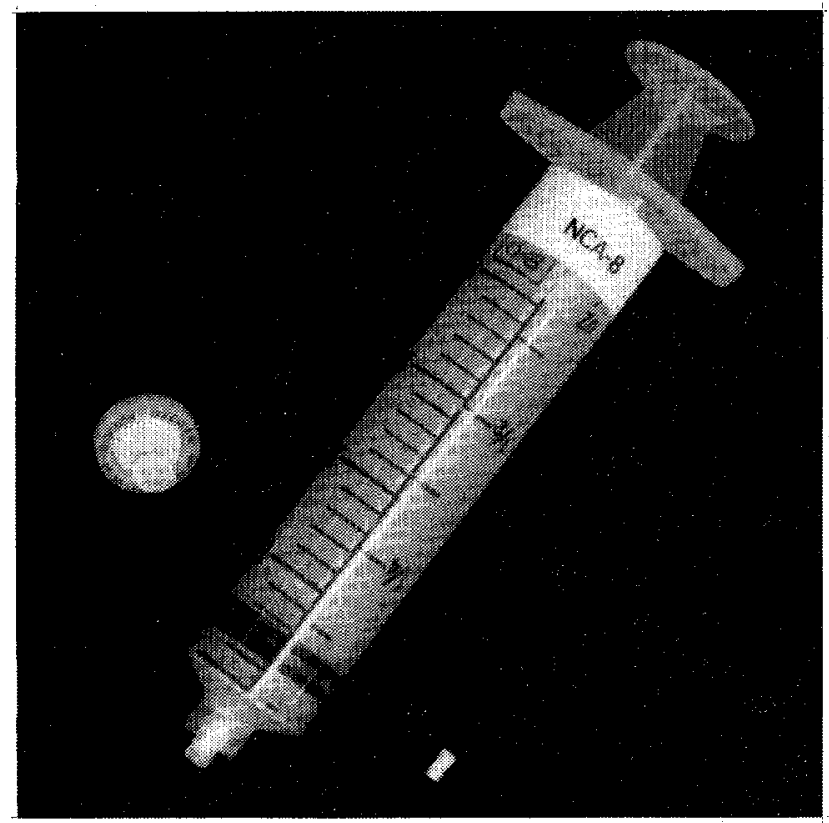

Fig. 1. Hypodermic syringe, with porous Kynar filter in tip, as used for solution/absorber contacts. An uninstalled Kynar filter is shown below the syringe.

The filter-tip syringes were unsuitable for the crystalline silico-titanate (CST) absorbers from Sandia National Laboratories because the CST particles were smaller than the pores in the Kynar filters. For these very finely divided materials, we achieved dynamic contact by placing each absorber/solution combination in a 20-mL centrifuge tube. At the end of each contact period, the tubes were centrifuged at high speed for $10 \mathrm{~min}$. We then transferred $1.55 \mathrm{~mL}$ of solution from each $30-\mathrm{mL}$ tube into a smaller centrifuge tube. The smaller tubes then were centrifuged at high speed for $10 \mathrm{~min}$. Finally, we transferred approximately $1.5 \mathrm{~mL}$ of solution from the smaller centrifuge tube into a $2.5-\mathrm{mL}$ hypodermic syringe with a $0.45-\mu \mathrm{m}$ filter (center left in Fig. 1) attached to the Luer-lock tip. The twice-centrifuged solution was expelled through this filter into a tared counting vial, from which we determined the solution weight.

The unusually large variation in Kd values obtained with the CST absorbers indicates that, in spite of our efforts to exclude them, some very small particles entered the solutions taken for gamma spectrometric assay. This problem was particularly apparent with strongly sorbed elements such as cesium. Even a few such particles on which cesium was strongly sorbed could have

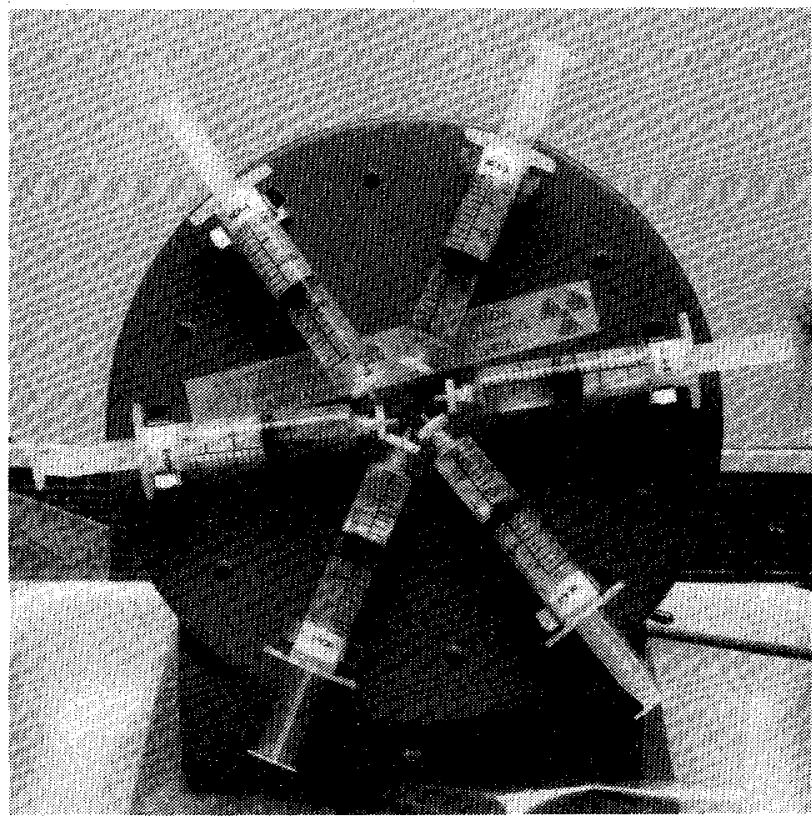

Fig. 2. Tube rotator used to mix the solution/ absorber combinations.

introduced a significant amount of activity into a nearly cesium-free solution. However, in all such cases the resulting error would cause us to understate the actual $\mathrm{Kd}$ values for removing cesium from solution. Our reported $\mathrm{Kd}$ values for the CST materials should, therefore, be considered as minimum values.

\section{E. Calculation of Kd Values}

Each portion of postcontact solution was assayed by gamma spectrometry for specific gamma energies of the added radionuclides. The fraction of each element sorbed was determined indirectly from the difference in the measured gamma activity of the selected radionuclide before and after contact with each specified absorber.

The Kd value for each element was calculated as follows:

$$
\mathrm{Kd}=\left(\frac{\mathrm{Pr}-\mathrm{Po}}{\mathrm{Po}}\right)\left(\frac{\mathrm{S}}{\mathrm{A}}\right),
$$

where $\operatorname{Pr}=$ measured precontact activity,

$\mathrm{Po}=$ measured postcontact activity,

$\mathrm{S}=$ milliliters of solution contacted, and

$\mathrm{A}=$ grams of dry absorber contacted. 
We recognize that uncertainties are larger when $\mathrm{Kd}$ values are very low (when the numerator represents a small difference between two large numbers), as well as when $\mathrm{Kd}$ values are very high (when the denominator is very small). However, the objective of this screening study was to identify absorbers that sorb specific elements poorly or very well. If it is necessary to determine Kd values with higher accuracy, such data can be obtained in follow-up studies, which would be limited to the most promising absorbers identified in this study.

Although we report most $\mathrm{Kd}$ values below 5000 as calculated, this obviously should not be taken to indicate that all four figures are significant. The uncertainty associated with our highest and lowest $K d$ values appears to be within a factor of 2 or 3 . We estimate the uncertainty associated with our best $\mathrm{Kd}$ values to range from 10 to 20 percent. To avoid implying more precision than is justified, we report Kd values above 5000 as greater than $(>)$ the next lower thousand $(K)$ value. In some cases where we feel the uncertainty is unusually high, even though the $\mathrm{Kd}$ value is less than 5000 , we report the $\mathrm{Kd}$ as greater than $(>)$ the next lower hundred value.

\section{F. Corrections}

The actinide-containing group of radiotracers exhibited direct interference between the gamma ray energies of two radionuclides. In addition to the measured 208-keV gamma ray, ${ }^{237} \mathrm{U}$ has a $59.5-\mathrm{keV}$ gamma whose energy coincides with that of the gamma we measured for ${ }^{241} \mathrm{Am}$. To correct for this interference, we determined the $59.5-\mathrm{keV} / 208-\mathrm{keV}$ ratio from a pure ${ }^{237} \mathrm{U}$ spectrum and used that ratio to correct the $59.5-\mathrm{keV}$ contribution from ${ }^{237} U$ in all assay portions (including precontact solutions) that contained both actinides.

Because the solution weight of the postcontact assay portion usually differed slightly from that of the precontact solution portion, each postcontact solution was normalized to the same weight basis as the precontact solution.

We also made appropriate corrections to account for the fact that the liquid/solid ratio and the remaining activity of every radionuclide decreased as each sequential assay portion was removed.

To determine the quantities of sorbed radionuclides, we compared the activity of each radionuclide in the postcontact solution with the activity of that same radio- nuclide in the precontact solution. Because most absorbers were initially added in air-dried form, the aqueous solvent was often preferentially sorbed, thereby decreasing the aqueous volume while simultaneously increasing the concentration of the solutes. Consequently, the activity of certain radionuclides was at times slightly higher in the postcontact solution than in the precontact solution. Whenever the postcontact/precontact solution activity ratio of a radionuclide exceeded unity, we assigned a value of 1.000 to the ratio having the highest value and normalized all other radionuclides in that same portion of solution accordingly.

We also made time-dependent corrections for the decay of ${ }^{237} U$ (6.8-day half-life) during the gamma assay period. We used an EXCEL ${ }^{\mathrm{TM}}$ spreadsheet to perform all $\mathrm{Kd}$ calculations and corrections.

\section{G. Other Sources of Error}

Each combination of element/absorber/solution/ contact time was measured only once. The absence of duplicates is somewhat offset by the fact that we measured individual $\mathrm{Kd}$ values for three consecutive contact periods. An abrupt change in the expected smooth trend of these three data points indicates an unreliable point.

Poor precision is also indicated by excessive variation within a set of three data points where no trend is evident. For very low or very high sorption, the variation is expected to be high for reasons already discussed.

At times, $\mathrm{Kd}$ values are highest for the 30 -min contact and decrease thereafter. Several scenarios could explain decreasing Kd values: (1) The element in question might sorb rapidly and then be displaced by another slower-sorbing species. (2) Some of the strongly complexing anions in the simulants could be participating in slow competitive reactions with the cations being measured. (3) The absorber could be uniformly or selectively dissolving, releasing previously sorbed cations in the process (slow dissolution of an amorphous phase within the crystalline silico-titanates may explain decreasing $\mathrm{Kd}$ values of some of the initially sorbed noncesium cations). (4) The decreasing Kd values could be entirely due to experimental error.

The difficulties in accurately correcting for the complex background under the low-energy (59.5-keV) gamma peak of ${ }^{241} \mathrm{Am}$ resulted in $\mathrm{Kd}$ values with unusually large uncertainties. 


\section{H. Data Transfer and Processing}

To save time and minimize the introduction of human errors inherent in manually transcribing large amounts of data, we automated the data transfer and calculation process. We used Gamanal ${ }^{9}$ to determine the area of each gamma peak of interest from the raw data in the multichannel analyzer memory. Gamanal provides a complete qualitative and quantitative analysis of mixtures of radionuclides by computer interpretation of high-resolution gamma spectra. Gamanal determines background, fits and resolves complex peak groupings, determines the energies and absolute intensities of the gamma rays, and calculates the quantities of the source radionuclides. All interferences are resolved by a leastsquares solution of the matrix of equations for the gamma intensities.

The Gamanal output was electronically imported into an EXCEL ${ }^{\mathrm{TM}}$ spreadsheet in which all described calculations and corrections were applied. Finally, all calculated $\mathrm{Kd}$ values were combined into one master spreadsheet and converted to a database to provide tabulated comparisons of the measured $\mathrm{Kd}$ values according to element, absorber, and solution type. Because small $\mathrm{Kd}$ values always have large associated uncertainties, we rounded all $\mathrm{Kd}$ values to not more than one place beyond the decimal point.

\section{Calculation of Detection Limits}

When peaks in a gamma spectrum were too small to be detected by the Gamanal program, we used a simple method $^{10}$ to calculate the appropriate detection limits. First, for each radionuclide of interest, the same detector was used to determine the peak position and full-width at half-maximum (FWHM) from a spectrum with a strong gamma signal. The FWHM then was doubled to include essentially the entire gamma-peak region (and always rounded up to the next integer) to define the position and width of the region of interest to be applied to the background spectra. The number of background counts in this region was summed, and the square root of the sum was multiplied by a confidence-limit scale factor. A scale-factor value of 2.772 was used to ensure that $95 \%$ of the signal peaks of this magnitude would be statistically detected on the observed background.
The scale factor was determined as follows. Counting statistics dictate that the square root of the number of counts in a region is an estimate of the standard deviation uncertainty of those counts. The detection of a peak requires that the background in the region of interest be subtracted; a single standard deviation of the difference is the square root of the sum of the squares of the singlestandard-deviation values for each of the two spectra. Thus, the standard deviation is the square root of the sums of the counts in the two corresponding regions of interest.

Because the hypothetical sample spectrum contains no detectable peak signal, the number of counts in the sample region of interest is equal to the number of counts in the background. Therefore, the single-standarddeviation uncertainty estimate (the confidence limits of detection) is the square root of twice the number of background counts, or 1.414 times the square root of the number of background counts. Scaling to exactly two standard deviations ( $95.44 \%$ confidence) would yield a scale factor of 2.828. Standard math tables permit the scale factor to be calculated to any desired confidence limit.

Using these calculated minimum peak area values that could be detected with a $95 \%$ confidence level to calculate the corresponding minimum $\mathrm{Kd}$ values, we report $\mathrm{Kd}$ values in terms of greater than $(>)$ the computed minimum $\mathrm{Kd}$ values.

\section{RESULTS AND DISCUSSION}

\section{A. Individual Elements}

Tables 5 through 18 list the most promising absorbers for each element, ranked in order of $\mathrm{Kd}$ value, for sorption from simulated acid-dissolved sludge, acidified supernate, and alkaline supernate solutions. Partitioning agents that sorb the specified element poorly are generally omitted from these tables, although absorbers designated as baseline technologies for the Hanford Tank Waste Remediation System (TWRS) are always included, even when they perform poorly. A complete listing of the $\mathrm{Kd}$ values for all elements is presented in section III.B, Individual Absorbers. 
1. Cerium. For sorption from acid-dissolved sludge solution, only the JSK experimental anion exchange resins outperform commercial Reillex ${ }^{\mathrm{TM}} \mathrm{HPQ}$ during short contact periods.
For sorption from acidified supernate solution, Cyanex ${ }^{\mathrm{TM}}$ 923, a low-cost commercial extractant, outperforms specialty products costing much more.

For sorption from alkaline solution, bone char and the Sandia absorbers offer high Kd values.

\begin{tabular}{|c|c|c|c|c|}
\hline \multirow[b]{2}{*}{ Solution } & \multirow[b]{2}{*}{ Absorber } & \multicolumn{3}{|c|}{ Kd Value for Specified Time } \\
\hline & & $30 \mathrm{~min}$ & $2 \mathrm{~h}$ & $6 \mathrm{~h}$ \\
\hline $\begin{array}{l}\text { Acid-Dissolved } \\
\text { Sludge }\end{array}$ & $\begin{array}{l}\text { JSK-1 resin } \\
\text { JSK-2 resin } \\
\text { JSK-3 resin } \\
\text { Reillex }^{\mathrm{TM}} \mathrm{HPQ} \\
\text { Purolite }^{\mathrm{TM}} \mathrm{A}-520-\mathrm{E} \\
\text { Ionac }^{\mathrm{TM}} \text { SR-3 } \\
\text { Crypt-DER }^{-D E} \\
\text { Duolite } \\
\text { Amberlyst }^{\mathrm{TM}} 167 \\
\end{array}$ & $\begin{array}{r}28 \\
27 \\
23 \\
14 \\
7.8 \\
6.6 \\
3.0 \\
2.4 \\
2.0 \\
\end{array}$ & $\begin{array}{l}29 \\
26 \\
23 \\
13 \\
14 \\
13 \\
2.9 \\
1.5 \\
1.7 \\
\end{array}$ & $\begin{array}{l}27 \\
24 \\
22 \\
13 \\
21 \\
21 \\
2.8 \\
2.2 \\
1.8 \\
\end{array}$ \\
\hline $\begin{array}{l}\text { Acidified } \\
\text { Supernate }\end{array}$ & $\begin{array}{l}\text { Cyanex }^{\mathrm{TM}} 923 \\
\text { TRU-Spec }^{\mathrm{TM}} \\
\text { SNL/HTO } \\
\text { CMPO-DIPB } \\
\text { Amberlyst }^{\mathrm{TM}} 15 \\
\text { LIX }^{\mathrm{TM}}-26 \\
\text { DHDECMP } \\
\text { MgO-PAN } \\
\text { MnO-PAN } \\
\text { Diphonix } \\
\text { Bone char } \\
\text { DHDECMP-DIPB } \\
\text { SNL/CST 111 } \\
\text { SRS resorcinol resin } \\
\text { Amberlyst } \\
\text { AM XN-1010 } \\
\text { AMP-PAN } \\
\text { SNL/CST } 68 \\
\text { SNL/CST } 48 \\
\text { Duolite } \\
\text { BM C-467 } \\
\text { Ba(Ca)SO }{ }_{4} \text {-PAN } \\
\text { Purolite }{ }^{\mathrm{TM}} \text { A-520-E } \\
\text { NaY-PAN } \\
\end{array}$ & $\begin{array}{r}744 \\
547 \\
265 \\
78 \\
42 \\
32 \\
26 \\
26 \\
24 \\
22 \\
19 \\
18 \\
17 \\
14 \\
13 \\
12 \\
8.6 \\
6.4 \\
6.3 \\
5.4 \\
4.8 \\
4.6 \\
\end{array}$ & $\begin{array}{r}988 \\
594 \\
320 \\
370 \\
77 \\
40 \\
45 \\
59 \\
25 \\
23 \\
33 \\
28 \\
32 \\
26 \\
38 \\
22 \\
16 \\
8.6 \\
4.3 \\
6.1 \\
5.3 \\
4.0 \\
\end{array}$ & $\begin{array}{r}1596 \\
644 \\
512 \\
499 \\
132 \\
42 \\
51 \\
222 \\
26 \\
22 \\
53 \\
28 \\
57 \\
42 \\
114 \\
28 \\
38 \\
17 \\
3.6 \\
8.6 \\
6.1 \\
3.3 \\
\end{array}$ \\
\hline $\begin{array}{l}\text { Alkaline } \\
\text { Supernate }\end{array}$ & $\begin{array}{l}\text { Bone char } \\
\text { SNL/CST } 149 \\
\text { SNL/CST } 69 \\
\text { SNL/HTO } \\
\text { SNL/CST } 141 \\
\text { SNL/CST } 35 \\
\text { SNL/CST } 111 \\
\text { SNL/CST } 120 \\
\text { LANL-TiP } \\
\text { MgO-PAN } \\
\text { Tannin } \\
\text { LANL-ZrP } \\
\text { M315-PAN } \\
\text { Ba(Ca)SO }-P A N\end{array}$ & $\begin{array}{r}914 \\
797 \\
205 \\
184 \\
177 \\
153 \\
139 \\
106 \\
30 \\
11 \\
9.4 \\
6.4 \\
5.3 \\
5.3\end{array}$ & $\begin{array}{r}>1400 \\
948 \\
>290 \\
>280 \\
199 \\
>300 \\
>300 \\
230 \\
61 \\
27 \\
13 \\
37 \\
13 \\
12\end{array}$ & $\begin{array}{r}>1500 \\
701 \\
298 \\
>290 \\
296 \\
191 \\
>300 \\
150 \\
22 \\
35 \\
22 \\
114 \\
53 \\
24\end{array}$ \\
\hline
\end{tabular}


2. Cesium. For sorption from all three solutions, the Sandia CSTs are among the best performers.

AMP-PAN sorbs cesium well from acid-dissolved sludge and acidified supernate solutions. NiFC-PAN also sorbs cesium well from acidified supernate; however, the decreasing $\mathrm{Kd}$ values in alkaline supernate are puzzling: The fact that other elements exhibit increasing $\mathrm{Kd}$ values on NiFC-PAN with longer contact times indicates that this absorber is not deteriorating.

For sorption from acidified supernate and alkaline supernate solutions, SRS resorcinol resin outperforms Duolite ${ }^{\mathrm{TM}} \mathrm{CS}-100$.

\begin{tabular}{|c|c|c|c|c|}
\hline \multirow[b]{2}{*}{ Solution } & \multirow[b]{2}{*}{ Absorber } & \multicolumn{3}{|c|}{ Kd Value for Specified Time } \\
\hline & & $30 \mathrm{~min}$ & $2 \mathbf{h}$ & $6 \mathrm{~h}$ \\
\hline $\begin{array}{l}\text { Acid-Dissolved } \\
\text { Sludge }\end{array}$ & $\begin{array}{l}\text { SNL/CST } 35 \\
\text { SNL/CST } 111 \\
\text { SNL/CST } 69 \\
\text { SNL/CST } 84 \\
\text { AMP-PAN } \\
\text { SNL/CST } 48 \\
\text { SNL/CST } 68 \\
\text { M315-PAN } \\
\text { NiFC-PAN } \\
\text { DurasilTM } 230 \\
\text { IonsivTM TIE-96 (mod.) } \\
\text { Ionsiv'TM TIE-96 } \\
\text { Duolite } \\
\text { TM } \text { CS-100 }\end{array}$ & $\begin{array}{r}4485 \\
3420 \\
2884 \\
1849 \\
1626 \\
794 \\
688 \\
50 \\
45 \\
11 \\
7.7 \\
7.6 \\
0.5\end{array}$ & $\begin{array}{r}1011 \\
>5 \mathrm{~K} \\
2279 \\
>8 \mathrm{~K} \\
4073 \\
758 \\
2307 \\
64 \\
49 \\
14 \\
9.0 \\
7.9 \\
1.1\end{array}$ & $\begin{array}{r}3416 \\
>5 \mathrm{~K} \\
3252 \\
>11 \mathrm{~K} \\
>5 \mathrm{~K} \\
1180 \\
1049 \\
61 \\
49 \\
19 \\
9.4 \\
8.3 \\
1.3\end{array}$ \\
\hline $\begin{array}{l}\text { Acidified } \\
\text { Supernate }\end{array}$ & $\begin{array}{l}\text { SNL/CST } 35 \\
\text { SNL/CST } 69 \\
\text { SNL/CST } 84 \\
\text { SNL/CST } 111 \\
\text { SNL/CST } 68 \\
\text { SNL/CST } 48 \\
\text { NiFC-PAN } \\
\text { AMP-PAN } \\
\text { M315-PAN } \\
\text { Durasil }{ }^{\mathrm{TM}} 230 \\
\text { SRS resorcinol resin } \\
\text { IonsivTM TIE-96 (mod.) } \\
\text { Ionsiv TM TIE-96 } \\
\text { SNL/HTO } \\
\text { Duolite } \\
\text { TM CS-100 }\end{array}$ & $\begin{array}{r}>13 \mathrm{~K} \\
>8 \mathrm{~K} \\
5484 \\
4938 \\
4072 \\
3951 \\
3533 \\
2133 \\
191 \\
78 \\
75 \\
63 \\
58 \\
10 \\
1.3\end{array}$ & $\begin{array}{r}>12 \mathrm{~K} \\
>10 \mathrm{~K} \\
3953 \\
2713 \\
2453 \\
2820 \\
>11 \mathrm{~K} \\
4636 \\
227 \\
135 \\
85 \\
110 \\
97 \\
8.9 \\
1.2 \\
\end{array}$ & $\begin{array}{r}4195 \\
>20 \mathrm{~K} \\
3098 \\
1864 \\
722 \\
1031 \\
>5 \mathrm{~K} \\
>8 \mathrm{~K} \\
231 \\
148 \\
66 \\
139 \\
122 \\
8.5 \\
1.3 \\
\end{array}$ \\
\hline $\begin{array}{l}\text { Alkaline } \\
\text { Supernate }\end{array}$ & $\begin{array}{l}\text { SNL/CST } 141 \\
\text { SNL/CST } 111 \\
\text { NiFC-PAN } \\
\text { SNL/CST } 149 \\
\text { CoFC-PSF } \\
\text { SNL/CST } 120 \\
\text { SRS resorcinol resin } \\
\text { Duolite }{ }^{\mathrm{TM}} \text { CS-100 } \\
\text { SNL/CST } 35 \\
\text { M315-PAN } \\
\text { DurasilT } 230 \\
\text { Tannin } \\
\text { TiFC-PSF } \\
\text { IonsivTM TIE-96 (mod.) } \\
\text { IonsivTM TIE-96 } \\
\text { Amberlyst TM XN-1010 } \\
\text { SNL/CST } 69 \\
\text { NaY-PAN }\end{array}$ & $\begin{array}{r}2521 \\
2323 \\
2090 \\
1866 \\
1154 \\
570 \\
314 \\
196 \\
165 \\
161 \\
66 \\
64 \\
59 \\
49 \\
47 \\
13 \\
10 \\
8.0\end{array}$ & $\begin{array}{r}2521 \\
2708 \\
943 \\
2402 \\
3793 \\
786 \\
599 \\
225 \\
277 \\
177 \\
106 \\
66 \\
102 \\
86 \\
84 \\
14 \\
13 \\
13 \\
7.8\end{array}$ & $\begin{array}{r}2312 \\
3076 \\
76 \\
3084 \\
3575 \\
898 \\
847 \\
233 \\
385 \\
177 \\
118 \\
66 \\
102 \\
107 \\
103 \\
15 \\
16 \\
7.5 \\
\end{array}$ \\
\hline
\end{tabular}


3. Strontium. Although Sr-Spec ${ }^{\mathrm{TM}}$ sorbs strontium from both acid-dissolved sludge and acidified supernate solutions better than the other absorbers, in neither case is the sorption very high.

For sorption from acidified supernate solution, Amberlyst ${ }^{\mathrm{TM}} 15$, whose cost is hundreds of times lower, performs nearly as well as Sr-Spec ${ }^{\mathrm{TM}}$. The poor to marginal performance of all the studied absorbers for sorbing strontium from these two solutions indicates that better partitioning agents are needed to remove this important fission product from acidic waste solutions.

For sorption from alkaline supernate solution, many absorbers offer excellent performance; the silicotitanates and hydrous titanium oxide from Sandia and the bone char are among the best.

\begin{tabular}{|c|c|c|c|c|}
\hline \multirow[b]{2}{*}{ Solution } & \multirow[b]{2}{*}{ Absorber } & \multicolumn{3}{|c|}{ Kd Value for Specified Time } \\
\hline & & $30 \mathrm{~min}$ & $2 \mathbf{h}$ & $6 \mathrm{~h}$ \\
\hline $\begin{array}{l}\text { Acid-Dissolved } \\
\text { Sludge }\end{array}$ & $\begin{array}{l}\text { Sr-Spec TM } \\
\text { Diphonix }{ }^{\mathrm{TM}} \\
\text { Ionsiviv TIE-96 } \\
\mathrm{Ba}(\mathrm{Ca}) \mathrm{SO}_{4} \text {-PAN } \\
\text { MgO-PAN } \\
\end{array}$ & $\begin{array}{l}3.0 \\
2.0 \\
1.9 \\
1.5 \\
1.4 \\
\end{array}$ & $\begin{array}{l}3.1 \\
1.7 \\
1.0 \\
1.7 \\
1.6\end{array}$ & $\begin{array}{l}2.9 \\
1.3 \\
1.5 \\
1.5 \\
2.0 \\
\end{array}$ \\
\hline $\begin{array}{l}\text { Acidified } \\
\text { Supernate }\end{array}$ & 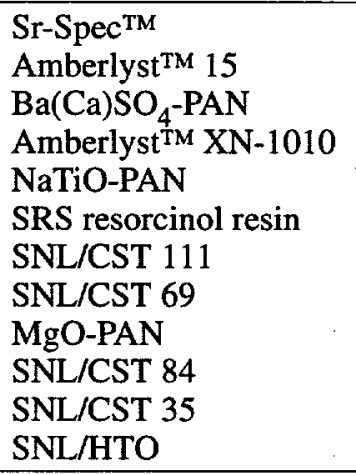 & $\begin{array}{l}18 \\
12 \\
7.3 \\
5.9 \\
4.9 \\
4.9 \\
4.2 \\
3.4 \\
3.0 \\
2.2 \\
1.6 \\
1.5\end{array}$ & $\begin{array}{l}18 \\
13 \\
8.1 \\
7.8 \\
5.3 \\
2.6 \\
4.2 \\
2.9 \\
4.8 \\
2.0 \\
1.3 \\
1.4\end{array}$ & $\begin{array}{r}17 \\
13 \\
12 \\
8.3 \\
5.0 \\
1.5 \\
3.5 \\
6.6 \\
7.4 \\
4.4 \\
3.3 \\
3.6 \\
\end{array}$ \\
\hline $\begin{array}{l}\text { Alkaline } \\
\text { Supernate }\end{array}$ & $\begin{array}{l}\text { SNL/CST } 149 \\
\text { Bone char } \\
\text { SNL/CST } 111 \\
\text { SNL/CST } 35 \\
\text { SNL/CST } 120 \\
\text { SNL/HTO } \\
\text { SNL/CST } 69 \\
\text { SNL/CST } 141 \\
\text { NiFC-PAN } \\
\text { Duolite }{ }^{\mathrm{TM}} \text { C-467 } \\
\text { TiO-PAN } \\
\text { Diphonix } \\
\text { MgO-PAN } \\
\text { NaTiO-PAN } \\
\text { IonsivTM TIE-96 (mod.) } \\
\text { TiFC-PSF } \\
\text { IonsivTM TIE-96 } \\
\text { CoFC-PSF } \\
\text { LANL-TiP } \\
\text { Ba(Ca)SO } 4 \text {-PAN } \\
\text { LANL-ZrO } \\
\text { Tannin } \\
\text { M315-PAN }\end{array}$ & $\begin{array}{r}>9 \mathrm{~K} \\
>5 \mathrm{~K} \\
4660 \\
>4200 \\
>4400 \\
>4000 \\
3839 \\
2755 \\
1121 \\
1051 \\
917 \\
546 \\
499 \\
314 \\
286 \\
144 \\
118 \\
84 \\
76 \\
76 \\
60 \\
50 \\
22\end{array}$ & $\begin{array}{r}>9 \mathrm{~K} \\
>11 \mathrm{~K} \\
>4500 \\
>4400 \\
>4500 \\
>4100 \\
1332 \\
>4300 \\
3736 \\
2734 \\
1627 \\
696 \\
768 \\
410 \\
405 \\
260 \\
140 \\
293 \\
117 \\
116 \\
164 \\
58 \\
165\end{array}$ & $\begin{array}{r}>9 \mathrm{~K} \\
>11 \mathrm{~K} \\
>4600 \\
>4600 \\
>4600 \\
>4200 \\
1659 \\
>4400 \\
4651 \\
>5 \mathrm{~K} \\
2856 \\
918 \\
689 \\
296 \\
421 \\
254 \\
159 \\
1270 \\
16 \\
146 \\
526 \\
102 \\
432\end{array}$ \\
\hline
\end{tabular}


4. Technetium. Reillex ${ }^{\mathrm{TM}} \mathrm{HPQ}$ offers the highest $\mathrm{Kd}$ value for sorbing technetium from acid-dissolved sludge and an even higher $\mathrm{Kd}$ value for sorption from acidified supernate solution. Although Reillex ${ }^{\mathrm{TM}} \mathrm{HPQ}$ provides Kd values of at least 250 for sorption from acidified supernate, two experimental resins and two commercial extractants outperform it.

Aliquat ${ }^{\mathrm{TM}} 336$ is especially interesting because it is nearly selective for sorbing technetium from acidified supernate and alkaline supernate solutions; moreover, its much lower Kd values for sorption from acid-dissolved sludge indicates that acid should be an effective stripping agent.

Most anion exchange resins sorb technetium well from acidic and alkaline solutions. Ionac ${ }^{\mathrm{TM}}$ SR-6, however, sorbs technetium well from acidified supernate and alkaline supernate solutions but not from acid-dissolved sludge solution. Thus, nitric acid may effectively elute technetium from this resin (see section IV, Elution Considerations).

\begin{tabular}{|c|c|c|c|c|}
\hline \multirow[b]{2}{*}{ Solution } & \multirow[b]{2}{*}{ Absorber } & \multicolumn{3}{|c|}{ Kd Value for Specified Time } \\
\hline & & $30 \mathrm{~min}$ & $2 \mathrm{~h}$ & $6 \mathrm{~h}$ \\
\hline \multirow[t]{2}{*}{ Acid-Dissolved } & Reillex $^{\mathrm{TM}} \mathrm{HPQ}$ & 76 & 90 & 93 \\
\hline & Ionac TM SR-3 & 32 & 44 & 43 \\
\hline \multirow[t]{12}{*}{ Sludge } & Purolite ${ }^{\mathrm{TM}}$ A-520-E & 32 & 57 & 77 \\
\hline & Sybron $^{\mathrm{TM}}(\mathrm{Et})_{3} \mathrm{~N}$ resin & 31 & 51 & 53 \\
\hline & LANL-TiO & 19 & 40 & 57 \\
\hline & LANL-ZrO & 17 & 41 & 60 \\
\hline & Duolite ${ }^{\text {TM }}$ C-467 & 12 & 12 & 13 \\
\hline & Sybron $^{\mathrm{TM}}(\mathrm{Pr})_{3} \mathrm{~N}$ resin & 11 & 26 & 45 \\
\hline & Tannin & 10 & 39 & 136 \\
\hline & LANL-NbO & 6.9 & 17 & 31 \\
\hline & Duolite $^{\text {TM }}$ CS -100 & 4.8 & 6.3 & 6.4 \\
\hline & Ionac ${ }^{\mathrm{TM}}$ SR-6 & 2.1 & 4.6 & 8.4 \\
\hline & SRS resorcinol resin & 2.1 & 4.6 & 11 \\
\hline & Aliquat ${ }^{\mathrm{TM}} 336$ & 0.7 & 0.8 & 1.0 \\
\hline Acidified & Sybron $^{\mathrm{TM}}(\mathrm{Pr})_{3} \mathrm{~N}$ resin & 401 & 777 & 992 \\
\hline \multirow{17}{*}{ Supernate } & Cyanex $^{\mathrm{TM}} 923^{3-}$ & 300 & 551 & 582 \\
\hline & Aliquat ${ }^{\mathrm{TM}} 336$ & 295 & 443 & 572 \\
\hline & Sybron $^{\mathrm{TM}}(\mathrm{Et})_{3} \mathrm{~N}$ resin & 264 & 405 & 440 \\
\hline & Reillex ${ }^{\mathrm{TM}} \mathrm{HPQ}$ & 250 & 280 & 274 \\
\hline & Purolite $^{\text {TM A-520-E }}$ & 205 & 320 & 380 \\
\hline & Ionac ${ }^{\mathrm{TM}}$ SR-6 & 151 & 394 & 618 \\
\hline & Ionac ${ }^{\mathrm{TM}}$ SR-3 & 121 & 123 & 125 \\
\hline & TRU-Spec $^{\text {TM }}$ & 90 & 78 & 74 \\
\hline & LANL-TiO & 54 & 185 & 290 \\
\hline & LIX ${ }^{\mathrm{TM}}-26$ & 54 & 112 & 190 \\
\hline & Sr-Spec ${ }^{\mathrm{TM}}$ & 45 & 42 & 39 \\
\hline & DHDECMP & 43 & 112 & 222 \\
\hline & CMPO-DIPB & 41 & 111 & 196 \\
\hline & Duolite ${ }^{\text {TM }}$ C-467 & 40 & 42 & 44 \\
\hline & LANL-ZrO & 35 & 148 & 258 \\
\hline & LANL-NbO & 28 & 134 & 271 \\
\hline & Crypt-DER & 25 & 25 & 25 \\
\hline
\end{tabular}


Table 8. Technetium Distribution Data (Cont.)

\begin{tabular}{|c|c|c|c|c|}
\hline \multirow{2}{*}{ Solution } & \multirow[b]{2}{*}{ Absorber } & \multicolumn{3}{|c|}{ Kd Value for Specified Tim } \\
\hline & & $30 \mathrm{~min}$ & $2 \mathrm{~h}$ & $6 \mathrm{~h}$ \\
\hline Alkaline & Aliquat $^{\text {TM }} 336$ & 417 & 614 & 651 \\
\hline \multirow[t]{16}{*}{ Supernate } & Purolite $^{\mathrm{TM}}$ A-520-E & 371 & 585 & 620 \\
\hline & Reillex ${ }^{\mathrm{TM}} \mathrm{HPQ}$ & 314 & 372 & 370 \\
\hline & $\operatorname{Sybron}^{\mathrm{TM}}(\mathrm{Et})_{3} \mathrm{~N}$ resin & 279 & 446 & 521 \\
\hline & Sybron $^{\mathrm{TM}}(\mathrm{Pr})_{3} \mathrm{~N}$ resin & 262 & 277 & 554 \\
\hline & Ionac TM SR-3 & 222 & 272 & 258 \\
\hline & Ionac ${ }^{\mathrm{TM}}$ SR-6 & 191 & 385 & 520 \\
\hline & Sr-Spec TM & 82 & 81 & 76 \\
\hline & CyanexтM 923 & 80 & 116 & 110 \\
\hline & Crypt-DER & 31 & 38 & 38 \\
\hline & DHDECMP & 21 & 23 & 21 \\
\hline & LIX ${ }^{\mathrm{TM}}-26$ & 21 & 23 & 22 \\
\hline & DHDECMP-DIPB & 14 & 14 & 13 \\
\hline & TRU-Spec TM & 7.6 & 7.5 & 7.2 \\
\hline & CMPO-DIPB & 4.5 & 6.8 & 7.6 \\
\hline & Tannin & 4.3 & 5.4 & 4.0 \\
\hline & Bone char & 2.9 & 5.1 & 4.9 \\
\hline
\end{tabular}


5. Yttrium. None of the absorbers sorbs yttrium well from acid-dissolved sludge solution.

From acidified supernate solution, Cyanex ${ }^{\mathrm{TM}}$ 923, a low-cost commercial extractant, sorbs yttrium at least tenfold better than much costlier alternatives.
From alkaline supernate solution, bone char sorbs yttrium best, although several other absorbers offer usable $\mathrm{Kd}$ values.

Table 9. Yttrium Distribution Data

\begin{tabular}{|c|c|c|c|c|}
\hline \multirow[b]{2}{*}{ Solution } & \multirow[b]{2}{*}{ Absorber } & \multicolumn{3}{|c|}{ Kd Value for Specified Time } \\
\hline & & $30 \mathrm{~min}$ & $2 \mathrm{~h}$ & $6 \mathrm{~h}$ \\
\hline \multirow{9}{*}{$\begin{array}{l}\text { Acid-Dissolved } \\
\text { Sludge }\end{array}$} & Duolite $^{\mathrm{TM}} \mathrm{C}-467$ & 1.8 & 0.9 & 1.6 \\
\hline & JSK-1 resin & 1.7 & 1.3 & 1.1 \\
\hline & JSK-2 resin & 1.6 & 1.7 & 1.4 \\
\hline & Ionac TM SR-3 & 1.5 & 1.7 & 1.4 \\
\hline & Ionsiv ${ }^{\mathrm{TM}}$ TIE-96 & 1.5 & 0.9 & 1.2 \\
\hline & Amberlyst ${ }^{\mathrm{TM}} \mathrm{XN}-1010$ & 1.4 & 1.4 & 1.5 \\
\hline & Purolite $^{\mathrm{TM}}$ A-520-E & 1.4 & 1.1 & 1.1 \\
\hline & Reillex $^{\mathrm{TM}} \mathrm{HPQ}$ & 1.2 & 0.5 & 0.8 \\
\hline & Tannin & 1.2 & 1.2 & 0.7 \\
\hline \multirow{11}{*}{$\begin{array}{l}\text { Acidified } \\
\text { Supernate }\end{array}$} & Cyanex $^{\text {TM }} 923$ & 1078 & 2014 & 2981 \\
\hline & TRU-Spec TM & 98 & 94 & 92 \\
\hline & SNL/HTO & 53 & 59 & 52 \\
\hline & Diphonix ${ }^{\mathrm{TM}}$ & 28 & 31 & 31 \\
\hline & Amberlyst $^{\mathrm{TM}} 15$ & 27 & 45 & 66 \\
\hline & MgO-PAN & 26 & 59 & 258 \\
\hline & CMPO-DIPB & 15 & 45 & 89 \\
\hline & AMP-PAN & 15 & 27 & 34 \\
\hline & SRS resorcinol resin & 12 & 21 & 36 \\
\hline & Bone char & 11 & 18 & 26 \\
\hline & Amberlyst ${ }^{\mathrm{TM}} \mathrm{XN}-1010$ & 11 & 26 & 64 \\
\hline \multirow{26}{*}{$\begin{array}{l}\text { Alkaline } \\
\text { Supernate }\end{array}$} & Bone char & 172 & 253 & 339 \\
\hline & SNL/CST 149 & 81 & 77 & 104 \\
\hline & NiFC-PAN & 48 & 72 & 99 \\
\hline & TiO-PAN & 39 & 64 & 102 \\
\hline & SNL/HTO & 37 & 68 & 93 \\
\hline & MgO-PAN & 35 & 58 & 98 \\
\hline & IonsivTM TIE-96 (mod.) & 28 & 41 & 47 \\
\hline & LANL-TiO & 20 & 31 & 20 \\
\hline & Duolite ${ }^{\mathrm{TM}} \mathrm{C}-467$ & 19 & 40 & 85 \\
\hline & NaTiO-PAN & 17 & 40 & 68 \\
\hline & MnO-PAN & 17 & 151 & 229 \\
\hline & Diphonix $^{\mathrm{TM}}$ & 16 & 20 & 28 \\
\hline & Ionsiv TM TIE-96 & 16 & 20 & 23 \\
\hline & SNL/CST 69 & 16 & 26 & 38 \\
\hline & $\mathrm{Ba}(\mathrm{Ca}) \mathrm{SO}_{4}-\mathrm{PAN}$ & 16 & 34 & 61 \\
\hline & SNL/CST 141 & 13 & 16 & 22 \\
\hline & Tannin & 12 & 18 & 34 \\
\hline & M315-PAN & 11 & 31 & 67 \\
\hline & Duolite $^{\text {TM }}$ CS-100 & 10 & 14 & 21 \\
\hline & NaY-PAN & 10 & 23 & 40 \\
\hline & TiFC-PSF & 9.7 & 25 & 37 \\
\hline & $\mathrm{LIX}^{\mathrm{TM}}-54$ & 9.3 & 24 & 56 \\
\hline & SNL/CST 35 & 8.9 & 17 & 37 \\
\hline & SNL/CST 120 & 8.3 & 12 & 21 \\
\hline & Sr-Spec TM & 7.9 & 8.5 & 6.7 \\
\hline & Durasil $^{\mathrm{TM}} 230$ & 7.3 & 9.8 & 11 \\
\hline
\end{tabular}


6. Chromium. None of the tested absorbers removes chromium adequately from acid-dissolved sludge solution.

For sorption from acidified supernate and alkaline supernate solutions, several absorbers offer double-digit $\mathrm{Kd}$ values after 6 -h contacts; however, the $\mathrm{Kd}$ values are significantly lower for shorter contact periods. The poor to marginal sorption of chromium by all the absorbers indicates that better partitioning agents are needed if this important matrix element is to be removed.

\begin{tabular}{|c|c|c|c|c|}
\hline \multirow[b]{2}{*}{ Solution } & \multirow[b]{2}{*}{ Absorber } & \multicolumn{3}{|c|}{ Kd Value for Specified Time } \\
\hline & & $30 \mathrm{~min}$ & $2 \mathrm{~h}$ & $6 \mathrm{~h}$ \\
\hline $\begin{array}{l}\text { Acid-Dissolved } \\
\text { Sludge }\end{array}$ & $\begin{array}{l}\text { MgO-PAN } \\
\text { LANL-ZrP } \\
\text { Ionsiv'TM TIE-96 } \\
\text { LANL-TiP } \\
\text { Ba(Ca)SO } \\
\text { Duolite }^{\mathrm{TM}} \text { C-46 } \\
\text { Diphonix } \\
\text { SNL/CST } 111 \\
\text { Amberlyst }^{\mathrm{TM}} 15 \\
\end{array}$ & $\begin{array}{l}1.5 \\
1.4 \\
1.4 \\
1.2 \\
1.1 \\
1.1 \\
1.1 \\
1.0 \\
1.0\end{array}$ & $\begin{array}{l}1.4 \\
1.1 \\
0.8 \\
1.1 \\
0.9 \\
0.4 \\
0.6 \\
1.0 \\
0.7\end{array}$ & $\begin{array}{l}1.6 \\
1.3 \\
1.1 \\
0.8 \\
0.9 \\
1.0 \\
0.8 \\
1.2 \\
0.9\end{array}$ \\
\hline $\begin{array}{l}\text { Acidified } \\
\text { Supernate }\end{array}$ & $\begin{array}{l}\text { SNL/HTO } \\
\text { Amberlyst }{ }^{\mathrm{TM}} 15 \\
\text { MgO-PAN } \\
\text { LANL-TiO } \\
\text { MnO-PAN } \\
\text { SNL/CST } 69 \\
\text { NaY-PAN } \\
\text { SNL/CST } 111 \\
\text { SNL/CST } 68 \\
\text { SNL/CST } 84 \\
\text { Sr-Spec } \\
\text { SNL/CST } 48 \\
\text { TRU-Spec } \\
\text { SNL/CST } 35 \\
\text { DuoliteTM }^{\mathrm{TM}} \text { CS-100 }\end{array}$ & $\begin{array}{l}5.9 \\
4.2 \\
4.0 \\
4.0 \\
3.0 \\
2.8 \\
2.3 \\
2.1 \\
1.7 \\
1.4 \\
1.3 \\
1.3 \\
1.3 \\
1.1 \\
1.0\end{array}$ & $\begin{array}{l}7.8 \\
7.9 \\
5.9 \\
4.4 \\
4.3 \\
3.8 \\
4.1 \\
1.9 \\
2.4 \\
1.7 \\
1.6 \\
1.7 \\
1.9 \\
1.9 \\
2.3\end{array}$ & \begin{tabular}{r|}
21 \\
14 \\
36 \\
5.4 \\
4.8 \\
14 \\
4.8 \\
1.7 \\
6.6 \\
8.8 \\
2.0 \\
6.0 \\
2.5 \\
9.6 \\
3.5 \\
\end{tabular} \\
\hline $\begin{array}{l}\text { Alkaline } \\
\text { Supernate }\end{array}$ & $\begin{array}{l}\text { SRS resorcinol resin } \\
\text { Tannin } \\
\mathrm{Ba}(\mathrm{Ca}) \mathrm{SO}_{4}-\mathrm{PAN}\end{array}$ & $\begin{array}{l}3.9 \\
1.9 \\
1.7\end{array}$ & $\begin{array}{l}16 \\
7.3 \\
2.2\end{array}$ & $\begin{array}{r}71 \\
27 \\
2.6\end{array}$ \\
\hline
\end{tabular}


7. Cobalt. Only tannin sorbs cobalt from acid-dissolved sludge solution with a double-digit $\mathrm{Kd}$ value, and achieving that requires a 6 -h contact period.

From acidified supernate solution, four partitioning agents sorb cobalt with double-digit Kd values; however, only $\mathrm{MgO}-\mathrm{PAN}$ offers a $\mathrm{Kd}$ value higher than 15 , and achieving that requires a 6 -h contact.
For sorption from alkaline supernate solution, five absorbers offer double-digit $\mathrm{Kd}$ values of borderline usefulness. More effective absorbers are needed for all three solutions if ${ }^{60} \mathrm{Co}$, an important activation product, is to be recovered.

\begin{tabular}{|c|c|c|c|c|}
\hline \multirow[b]{2}{*}{ Solution } & \multirow[b]{2}{*}{ Absorber } & \multicolumn{3}{|c|}{ Kd Value for Specified Time } \\
\hline & & 30 min & $2 \mathbf{h}$. & $6 \mathrm{~h}$ \\
\hline $\begin{array}{l}\text { Acid-Dissolved } \\
\text { Sludge }\end{array}$ & $\begin{array}{l}\text { Diphonix }^{\mathrm{TM}} \\
\text { MgO-PAN } \\
\text { Duolite }{ }^{\mathrm{TM}} \mathrm{C}-467 \\
\text { M315-PAN } \\
\text { LANL-ZrP } \\
\text { Tannin } \\
\text { Amberlyst } \\
\text { Ionsiv } \mathrm{XN} \text {-1010 } \\
\text { TIE-96 }\end{array}$ & $\begin{array}{l}1.4 \\
1.3 \\
1.3 \\
1.3 \\
1.3 \\
1.3 \\
1.3 \\
1.2 \\
\end{array}$ & $\begin{array}{l}1.7 \\
1.4 \\
0.4 \\
1.0 \\
1.3 \\
3.5 \\
0.9 \\
0.7\end{array}$ & $\begin{array}{r}2.1 \\
1.4 \\
0.8 \\
1.1 \\
1.1 \\
13 \\
1.2 \\
0.8 \\
\end{array}$ \\
\hline $\begin{array}{l}\text { Acidified } \\
\text { Supernate }\end{array}$ & $\begin{array}{l}\text { Duolite }^{\mathrm{TM}} \text { C-467 } \\
\text { SRS resorcinol resin } \\
\text { MgO-PAN } \\
\text { NiFC-PAN } \\
\text { NaTiO-PAN } \\
\text { SNL/CST } 68 \\
\text { SNL/CST } 48 \\
\text { Bone char } \\
\text { Amberlyst } \\
\text { Amberlyst } \mathrm{XN}-1010 \\
\text { Crypt-DER }\end{array}$ & $\begin{array}{l}14 \\
12 \\
9.4 \\
8.9 \\
6.2 \\
5.3 \\
4.5 \\
4.4 \\
4.0 \\
3.6 \\
2.1\end{array}$ & $\begin{array}{r}13 \\
15 \\
15 \\
10 \\
7.9 \\
5.4 \\
4.6 \\
5.5 \\
3.5 \\
3.5 \\
2.1\end{array}$ & $\begin{array}{r}12 \\
7.7 \\
33 \\
11 \\
9.5 \\
2.3 \\
2.0 \\
6.9 \\
3.4 \\
3.1 \\
2.1 \\
\end{array}$ \\
\hline $\begin{array}{l}\text { Alkaline } \\
\text { Supernate }\end{array}$ & $\begin{array}{l}\text { Bone char } \\
\text { Duolite }{ }^{\mathrm{TM}} \text { CS-100 } \\
\text { LANL-TiO } \\
\text { NiFC-PAN } \\
\text { LANL-NbO } \\
\text { LANL-ZrO } \\
\text { SRS resorcinol resin }\end{array}$ & $\begin{array}{l}14 \\
8.1 \\
6.4 \\
5.7 \\
5.7 \\
4.3 \\
2.9\end{array}$ & $\begin{array}{r}17 \\
12 \\
12 \\
7.0 \\
15 \\
7.6 \\
7.5\end{array}$ & $\begin{array}{r}18 \\
16 \\
18 \\
8.4 \\
22 \\
10 \\
13\end{array}$ \\
\hline
\end{tabular}


8. Iron. Iron is poorly sorbed from both acid-dissolved sludge and acidified supernate solutions by all absorbers. Because iron is present at high concentrations in many waste solutions, we had expected it to interfere with the sorption of other elements whose removal is critical to the HLW cleanup effort. However, its low $\mathrm{Kd}$ values indicate that its effect on other elements may be less than anticipated. Nevertheless, when iron is present at high concentration, significant amounts may load even on absorbers with low Kd values for iron.

The high $\mathrm{Kd}$ values for sorbing iron from alkaline supernate on many absorbers may preclude their use for sorbing other elements of interest.

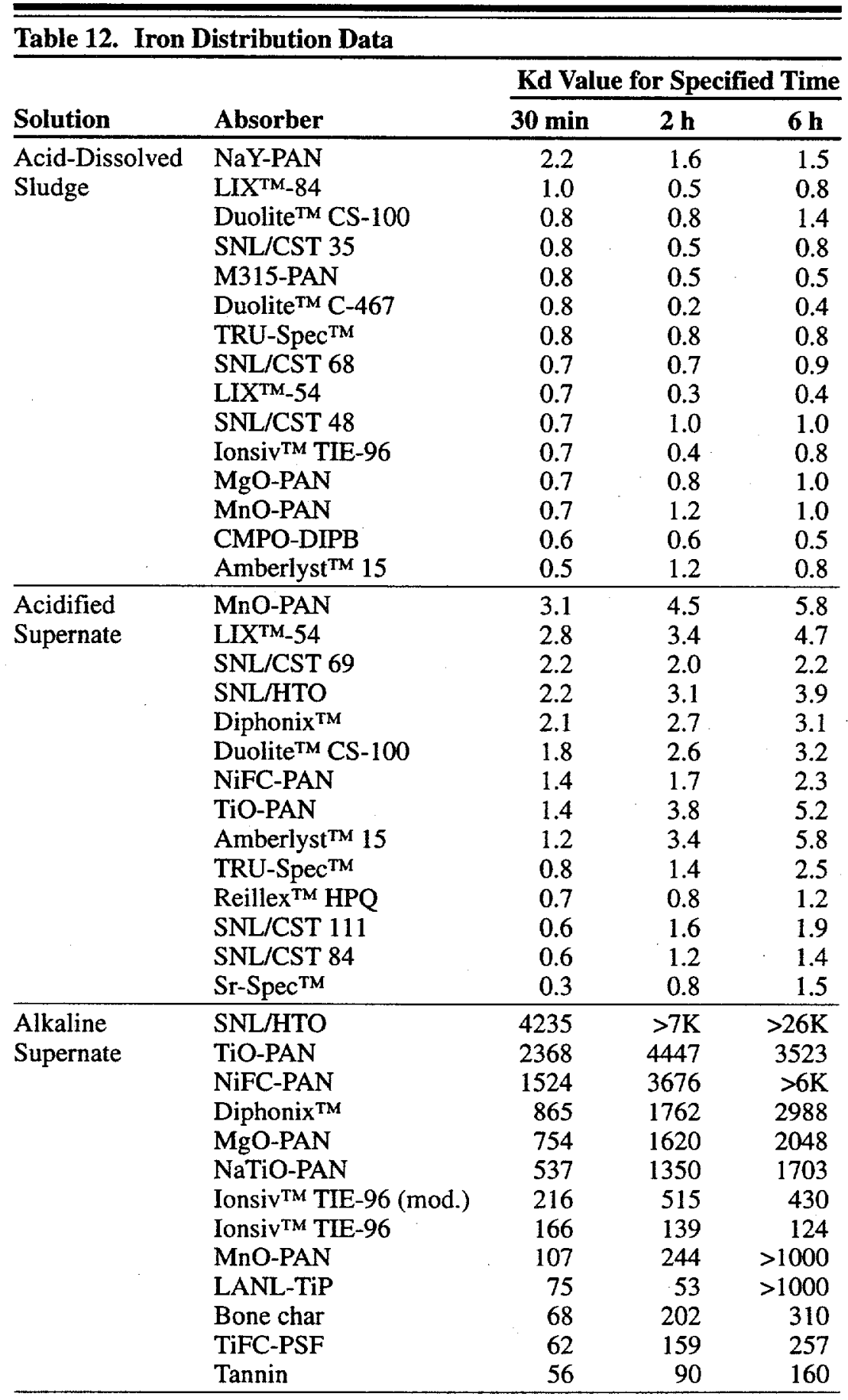




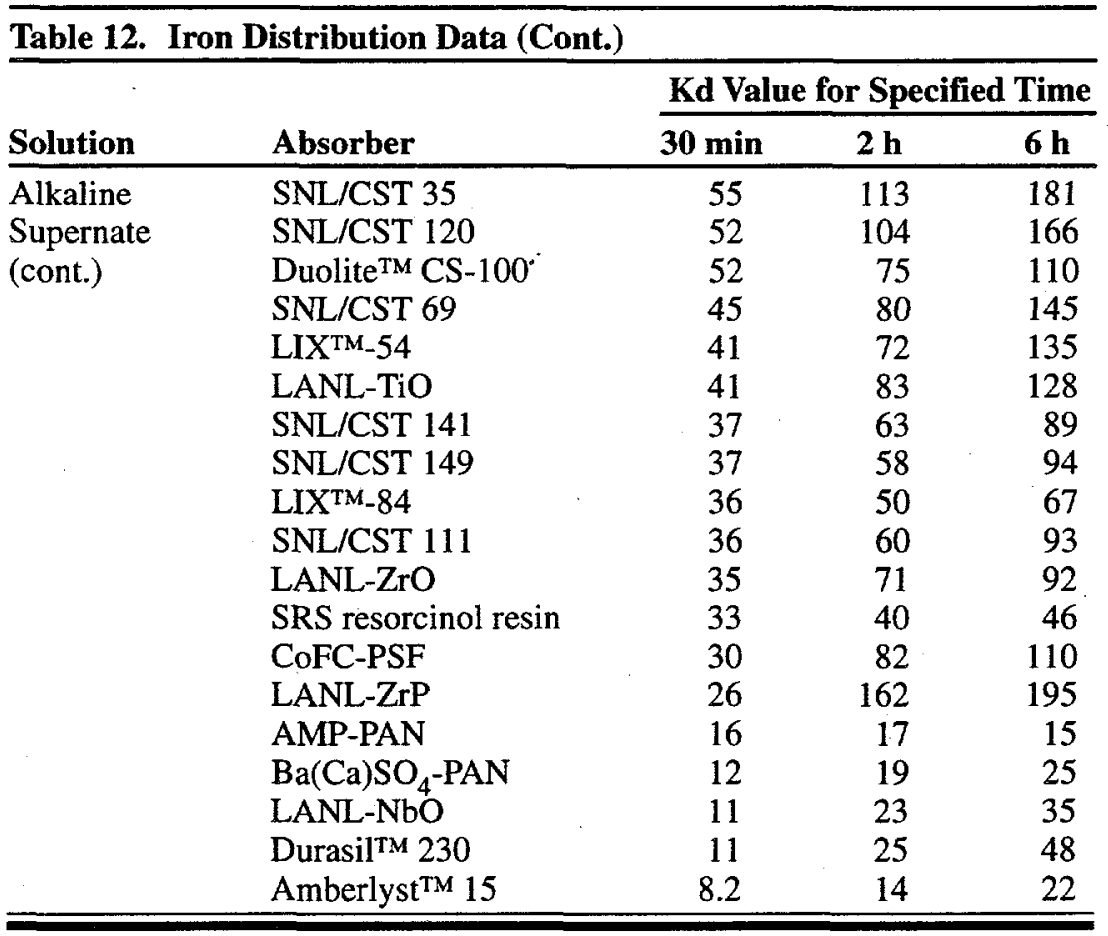


9. Manganese. Manganese is moderately sorbed from acidified supernate solution by Duolite ${ }^{\mathrm{TM}} \mathrm{C}-467$ and NaTiOPAN but is not sorbed from acid-dissolved sludge by any of the absorbers tested in this study. As in the case of iron, this suggests that the risk of interference posed by the competitive sorption of manganese from these solutions may be less than expected.

For sorption from alkaline supernate solution, many absorbers offer high Kd values for manganese. Thus, these absorbers may be useless for removing other elements of greater interest from this solution.

\begin{tabular}{|c|c|c|c|c|}
\hline \multirow[b]{2}{*}{ Solution } & \multirow[b]{2}{*}{ Absorber } & \multicolumn{3}{|c|}{ Kd Value for Specified Time } \\
\hline & & $30 \mathrm{~min}$ & $2 \mathrm{~h}$ & $6 \mathrm{~h}$ \\
\hline $\begin{array}{l}\text { Acid-Dissolved } \\
\text { Sludge }\end{array}$ & $\begin{array}{l}\text { Diphonix } \\
\text { Duolite }^{\mathrm{TM}} \text { C-467 } \\
\text { Amberlyst }^{\mathrm{TM}} \mathrm{XN}-1010 \\
\text { PK-01-244 resin } \\
\text { LIX'84 } \\
\text { Crypt-DER } \\
\text { NaTiO-PAN }\end{array}$ & $\begin{array}{l}1.8 \\
1.3 \\
1.1 \\
1.0 \\
0.9 \\
0.8 \\
0.4\end{array}$ & $\begin{array}{l}3.2 \\
1.3 \\
0.5 \\
0.3 \\
0.3 \\
0.7 \\
0.4\end{array}$ & $\begin{array}{l}3.8 \\
1.4 \\
0.4 \\
0.3 \\
0.5 \\
0.4 \\
0.4\end{array}$ \\
\hline $\begin{array}{l}\text { Acidified } \\
\text { Supernate }\end{array}$ & $\begin{array}{l}\text { Duolite }^{\mathrm{TM}} \text { C-467 } \\
\text { NaTiO-PAN } \\
\text { SRS resorcinol resin } \\
\text { Amberlyst }^{\mathrm{TM}} 15 \\
\text { LIX }^{\mathrm{TM}}-1010 \\
\text { Amberlyst }^{\mathrm{TM}} \text { XN-1010 } \\
\text { LIX }^{\mathrm{TM}} \text {-54 } \\
\text { SNL/CST } 48 \\
\text { SNL/CST } 68 \\
\text { Bone char } \\
\text { MnO-PAN } \\
\text { CMPO-DIPB } \\
\text { Tannin } \\
\end{array}$ & $\begin{array}{l}15 \\
6.0 \\
5.9 \\
5.2 \\
4.9 \\
4.3 \\
2.4 \\
2.0 \\
1.9 \\
1.8 \\
1.8 \\
1.6 \\
1.3\end{array}$ & $\begin{array}{l}17 \\
7.3 \\
4.7 \\
4.7 \\
5.7 \\
4.9 \\
2.1 \\
1.7 \\
1.9 \\
2.5 \\
1.9 \\
1.6 \\
0.4\end{array}$ & $\begin{array}{l}17 \\
7.6 \\
2.2 \\
4.5 \\
5.3 \\
5.0 \\
2.6 \\
1.2 \\
1.6 \\
2.9 \\
2.2 \\
2.1 \\
0.5 \\
\end{array}$ \\
\hline $\begin{array}{l}\text { Alkaline } \\
\text { Supernate }\end{array}$ & 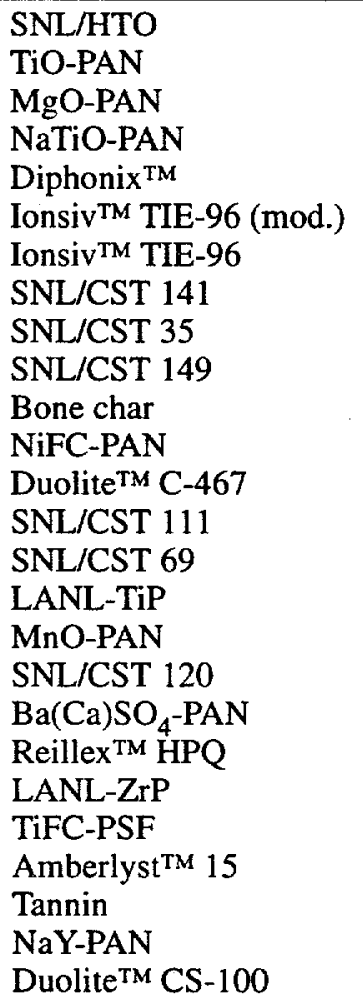 & $\begin{array}{r}1969 \\
774 \\
172 \\
142 \\
128 \\
121 \\
103 \\
102 \\
95 \\
69 \\
62 \\
59 \\
52 \\
52 \\
50 \\
47 \\
25 \\
24 \\
17 \\
14 \\
12 \\
9.9 \\
8.3 \\
7.1 \\
6.9 \\
4.8\end{array}$ & $\begin{array}{r}4777 \\
1859 \\
373 \\
319 \\
200 \\
280 \\
115 \\
178 \\
375 \\
134 \\
927 \\
167 \\
80 \\
109 \\
101 \\
49 \\
76 \\
117 \\
58 \\
18 \\
106 \\
33 \\
13 \\
16 \\
14 \\
9.7\end{array}$ & $\begin{array}{r}>9 \mathrm{~K} \\
3110 \\
623 \\
616 \\
296 \\
322 \\
126 \\
275 \\
635 \\
235 \\
3963 \\
363 \\
93 \\
225 \\
167 \\
>1000 \\
>1000 \\
215 \\
104 \\
26 \\
96 \\
87 \\
21 \\
34 \\
28 \\
14\end{array}$ \\
\hline
\end{tabular}


10. Zinc. None of the absorbers removes significant amounts of zinc from acid-dissolved sludge solution.

Several absorbers offer usable Kd values for sorption from acidified supernate solution.
From alkaline supernate solution, many absorbers offer very high sorption of zinc. The observed $\mathrm{pH}$ effect suggests that zinc could easily be sorbed from alkaline solution and eluted with acid.

\begin{tabular}{|c|c|c|c|c|}
\hline \multirow[b]{2}{*}{ Solution } & \multirow[b]{2}{*}{ Absorber } & \multicolumn{3}{|c|}{ Kd Value for Specified Time } \\
\hline & & $30 \mathrm{~min}$ & $2 \mathrm{~h}$ & $6 \mathrm{~h}$ \\
\hline $\begin{array}{l}\text { Acid-Dissolved } \\
\text { Sludge }\end{array}$ & $\begin{array}{l}\text { JSK-2 resin } \\
\text { JSK-3 resin } \\
\text { JSK-1 resin } \\
\text { Diphonix } \\
\text { Duolite }{ }^{\text {TM }} \text { C-467 } \\
\text { PK-01-244 resin } \\
\text { SNL/CST } 111 \\
\text { Crypt-DER resin }\end{array}$ & $\begin{array}{l}2.8 \\
1.7 \\
1.6 \\
1.2 \\
0.9 \\
0.8 \\
0.8 \\
0.7 \\
\end{array}$ & $\begin{array}{l}1.9 \\
1.3 \\
1.0 \\
2.1 \\
0.7 \\
0.4 \\
0.6 \\
0.5 \\
\end{array}$ & $\begin{array}{l}1.1 \\
0.7 \\
0.9 \\
2.1 \\
0.8 \\
0.4 \\
0.6 \\
0.3 \\
\end{array}$ \\
\hline $\begin{array}{l}\text { Acidified } \\
\text { Supernate }\end{array}$ & $\begin{array}{l}\text { NiFC-PAN } \\
\text { SNL/CST } 68 \\
\text { DuoliteTM C-467 } \\
\text { SNL/CST } 48 \\
\text { NaTiO-PAN } \\
\text { SNL/HTO } \\
\text { SRS resorcinol resin } \\
\text { Bone char } \\
\text { LIXTM-1010 }^{\text {Amberlyst }}{ }^{\mathrm{TM}} \text { XN-1010 }\end{array}$ & $\begin{array}{r}61 \\
33 \\
24 \\
23 \\
21 \\
18 \\
9.3 \\
8.3 \\
3.6 \\
3.2 \\
\end{array}$ & $\begin{array}{l}94 \\
44 \\
46 \\
32 \\
44 \\
18 \\
11 \\
14 \\
4.0 \\
3.4 \\
\end{array}$ & $\begin{array}{r}90 \\
43 \\
89 \\
34 \\
71 \\
19 \\
9.0 \\
18 \\
4.0 \\
3.6 \\
\end{array}$ \\
\hline $\begin{array}{l}\text { Alkaline } \\
\text { Supernate }\end{array}$ & $\begin{array}{l}\text { SNL/HTO } \\
\text { NiFC-PAN } \\
\text { TiO-PAN } \\
\text { MgO-PAN } \\
\text { MnO-PAN } \\
\text { Diphonix } \\
\text { Duolite } \\
\text { LAN C-467 } \\
\text { Duolite }{ }^{\mathrm{TM}} \text { CS-100 } \\
\text { NaTiO-PAN } \\
\text { Bone char } \\
\text { Tannin } \\
\text { LANL-TiO } \\
\text { Ionsiv TM TIE-96 } \\
\text { AMP-PAN } \\
\text { TiFC-PSF } \\
\text { IonsivTM TIE-96 (mod.) } \\
\text { LANL-ZrO } \\
\text { CoFC-PSF } \\
\text { DurasilTM 230 } \\
\text { LANL-ZrP }\end{array}$ & $\begin{array}{r}4625 \\
3800 \\
2875 \\
2051 \\
1519 \\
792 \\
292 \\
173 \\
122 \\
115 \\
86 \\
60 \\
51 \\
49 \\
34 \\
30 \\
29 \\
19 \\
11 \\
12 \\
8.8\end{array}$ & $\begin{array}{r}>11 \mathrm{~K} \\
>11 \mathrm{~K} \\
>6 \mathrm{~K} \\
4193 \\
3806 \\
1616 \\
617 \\
118 \\
286 \\
540 \\
153 \\
143 \\
215 \\
130 \\
37 \\
211 \\
96 \\
37 \\
85 \\
23 \\
30\end{array}$ & $\begin{array}{r}25 \mathrm{~K} \\
>17 \mathrm{~K} \\
>9 \mathrm{~K} \\
4040 \\
>10 \mathrm{~K} \\
2406 \\
910 \\
>1000 \\
490 \\
1117 \\
215 \\
251 \\
448 \\
227 \\
29 \\
335 \\
198 \\
53 \\
471 \\
35 \\
104\end{array}$ \\
\hline
\end{tabular}


11.Zirconium. Many absorbers offer moderate to high sorption of zirconium from acid-dissolved sludge solution. Of particular interest is TiO-PAN, which is highly selective for zirconium. We attribute the erratic Kd values for SNL/ CST 69 to incomplete exclusion of very small absorber particles from the assay solution, and those for SNL/CST 84 to dissolution of an amorphous phase that appears to preferentially sorb zirconium.
For sorption from acidified supernate solution, only MgO-PAN offers a double-digit Kd value for zirconium. However, because the $\mathrm{Kd}$ value for MgO-PAN for a contact time of $6 \mathrm{~h}$ is significantly higher than values obtained for shorter contact times, this high value is suspect.

Many absorbers offer very high sorption of zirconium from alkaline supernate solution. Because zirconium is a major matrix element in many HLW solutions, it may present serious interference problems for the cited absorbers.

Table 15. Zirconium Distribution Data

\begin{tabular}{|c|c|c|c|c|}
\hline \multirow{2}{*}{ Solution } & \multirow[b]{2}{*}{ Absorber } & \multicolumn{3}{|c|}{ Kd Value for Specified Time } \\
\hline & & $30 \mathrm{~min}$ & $2 \mathrm{~h}$ & $6 \mathrm{~h}$ \\
\hline Acid-Dissolved & NaY-PAN & 107 & 191 & 196 \\
\hline \multirow[t]{13}{*}{ Sludge } & SNL/CST 69 & 91 & 14 & 116 \\
\hline & Durasil $^{\mathrm{TM}} 230$ & 75 & 220 & 205 \\
\hline & SNL/CST 84 & 70 & 87 & 19 \\
\hline & TiO-PAN & 51 & 323 & 899 \\
\hline & IonsivTM TIE-96 (mod.) & 35 & 88 & 115 \\
\hline & SNL/CST 68 & 31 & 119 & 103 \\
\hline & Ionsiv'M TIE-96 & 28 & 64 & 85 \\
\hline & M315-PAN & 27 & 67 & 96 \\
\hline & SNL/CST 48 & 15 & 49 & 51 \\
\hline & SNL/CST 111 & 11 & 14 & 18 \\
\hline & Duolite ${ }^{\mathrm{TM}}$ CS-100 & 9.2 & 16 & 22 \\
\hline & SNL/CST 35 & 8.2 & 26 & 76 \\
\hline & Cyanex ${ }^{\mathrm{TM}} 272$ & 8.0 & 32 & 199 \\
\hline Acidified & Amberlyst $^{\mathrm{TM}} 15$ & 2.8 & 4.6 & 8.4 \\
\hline \multirow{8}{*}{ Supernate } & MgO-PAN & 1.7 & 2.9 & 28 \\
\hline & SNL/HTO & 1.7 & 2.7 & 6.4 \\
\hline & SNL/CST 69 & 1.7 & 2.5 & 5.5 \\
\hline & MnO-PAN & 1.7 & 2.2 & 2.3 \\
\hline & Sr-Spec TM & 1.6 & 1.8 & 2.1 \\
\hline & TiO-PAN & 1.5 & 2.0 & 3.2 \\
\hline & TRU-Spec ${ }^{\mathrm{TM}}$ & 1.4 & 1.9 & 2.6 \\
\hline & Duolite ${ }^{\text {TM }}$ CS-100 & 1.1 & 2.1 & 3.1 \\
\hline
\end{tabular}


Table 15. Zirconium Distribution Data (Cont.)

\begin{tabular}{|c|c|c|c|c|}
\hline \multirow{2}{*}{ Solution } & \multirow[b]{2}{*}{ Absorber } & \multicolumn{3}{|c|}{ Kd Value for Specified Time } \\
\hline & & $30 \mathrm{~min}$ & $2 \mathrm{~h}$ & $6 \mathrm{~h}$ \\
\hline Alkaline & SNL/HTO & 1037 & 2220 & 4276 \\
\hline \multirow{28}{*}{ Supernate } & TiO-PAN & 615 & 1187 & 2092 \\
\hline & Diphonix $^{\mathrm{TM}}$ & 359 & 759 & 927 \\
\hline & MnO-PAN & 270 & 925 & 1543 \\
\hline & MgO-PAN & 233 & 460 & 643 \\
\hline & Bone char & 204 & 339 & 506 \\
\hline & NaTiO-PAN & 189 & 526 & 833 \\
\hline & NiFC-PAN & 179 & 375 & 474 \\
\hline & $\mathrm{Ba}(\mathrm{Ca}) \mathrm{SO}_{4}-\mathrm{PAN}$ & 161 & 327 & 482 \\
\hline & SNL/CST 120 & 147 & 283 & 449 \\
\hline & Ionsiv TM TIE-96 (mod.) & 146 & 379 & 327 \\
\hline & Duolite ${ }^{\mathrm{TM}} \mathrm{CS}-100$ & 142 & 222 & 304 \\
\hline & Tannin & 131. & 231 & 372 \\
\hline & IonsivTM TIE-96 & 117 & 151 & 129 \\
\hline & SNL/CST 35 & 113 & 280 & 504 \\
\hline & SNL/CST 149 & 94 & 104 & 95 \\
\hline & LANL-TiP & 88 & 106 & 34 \\
\hline & SNL/CST 111 & 83 & 85 & 68 \\
\hline & TiFC-PSF & 73 & 192 & 222 \\
\hline & SNL/CST 141 & 64 & 62 & 53 \\
\hline & LANL-ZrO & 58 & 273 & 611 \\
\hline & SRS resorcinol resin & 41 & 81 & 123 \\
\hline & LANL-TiO & 35 & 205 & 570 \\
\hline & Durasil' 230 & 35 & 86 & 148 \\
\hline & SNL/CST 69 & 32 & 87 & 141 \\
\hline & M315-PAN & 19 & 34 & 50 \\
\hline & LANL-ZrP & 18 & 52 & 159 \\
\hline & CoFC-PSF & 13 & 46 & 129 \\
\hline & NaY-PAN & 12 & 18 & 21 \\
\hline
\end{tabular}


12. Uranium. Many absorbers offer moderate $\mathrm{Kd}$ values for sorbing uranium from acid-dissolved sludge solution. Note that all 60 absorbers were evaluated for sorbing uranium from acid-dissolved sludge that contained $3 \mathrm{~g}{ }^{239} \mathrm{Pu} / \mathrm{L}$. A few selected absorbers also were evaluated for sorbing uranium from a similar solution that contained ${ }^{238} \mathrm{Pu}$ at a 50-fold lower concentration to determine how the lower plutonium concentration affected the $\mathrm{Kd}$ values of other actinides.
For sorbing uranium from acidified supernate solution, Cyanex ${ }^{\mathrm{TM}} 923$ outperforms other much costlier partitioning agents.

For sorbing uranium from alkaline supernate solution, TiO-PAN and SNL/HTO offer very high Kd values, whereas numerous other absorbers offer moderate to high Kd values.

\begin{tabular}{|c|c|c|c|c|}
\hline \multirow[b]{2}{*}{ Solution } & \multirow[b]{2}{*}{ Absorber } & \multicolumn{3}{|c|}{ Kd Value for Specified Time } \\
\hline & & $30 \mathrm{~min}$ & $2 \mathrm{~h}$ & $6 \mathrm{~h}$ \\
\hline Acid-Dissolved & TRU-Spec TM & 25 & 26 & 27 \\
\hline \multirow{13}{*}{ Sludge, $3 \mathrm{~g} \mathrm{Pu} / \mathrm{L}$} & JSK-2 resin & 19 & 19 & 19 \\
\hline & JSK-3 resin & 18 & 18 & 17 \\
\hline & Reillex ${ }^{\mathrm{TM}} \mathrm{HPQ}$ & 16 & 19 & 18 \\
\hline & JSK-1 resin & 14 & 13 & 12 \\
\hline & Sybron $^{\mathrm{TM}}(\mathrm{Et})_{3} \mathrm{~N}$ resin & 13 & 22 & 22 \\
\hline & Ionac TM SR-3 & 12 & 21 & 37 \\
\hline & Purolite $^{\mathrm{TM}}$ A-520-E & 9.9 & 24 & 35 \\
\hline & Sr-Spec ${ }^{\mathrm{TM}}$ & 4.9 & 5.1 & 5.7 \\
\hline & Duolite ${ }^{\mathrm{TM}} \mathrm{C}-467$ & 3.8 & 5.0 & 6.0 \\
\hline & Amberlyst ${ }^{\mathrm{TM}} 15$ & 3.1 & 6.4 & 7.2 \\
\hline & DHDECMP-DIPB & 1.2 & 2.3 & 2.7 \\
\hline & Diphonix $^{\mathrm{TM}}$ & 0.4 & 1.5 & 2.8 \\
\hline & CMPO-DIPB & 0.4 & 0.8 & 1.4 \\
\hline \multirow{6}{*}{$\begin{array}{l}\text { Acid-Dissolved } \\
\text { Sludge, } 60 \mathrm{mg} \mathrm{Pu} / \mathrm{L}\end{array}$} & Reillex $^{\mathrm{TM}} \mathrm{HPQ}$ & 20 & 23 & 23 \\
\hline & JSK-2 resin & 20 & 17 & 17 \\
\hline & JSK-3 resin & 18 & 17 & 17 \\
\hline & JSK-1 resin & 13 & 12 & 11 \\
\hline & Purolite ${ }^{\mathrm{TM}}$ A-520-E & 10 & 22 & 39 \\
\hline & Cyanex ${ }^{\mathrm{TM}} 923$ & 2.7 & 6.5 & 14 \\
\hline Acidified & Cyanex TM 923 & 122 & 233 & 356 \\
\hline \multirow[t]{16}{*}{ Supernate } & Diphonix ${ }^{\mathrm{TM}}$ & 96 & 153 & 214 \\
\hline & TRU-Spec TM & 70 & 107 & 164 \\
\hline & TiO-PAN & 67 & 115 & 185 \\
\hline & SNL/HTO & 52 & 69 & 90 \\
\hline & CMPO-DIPB & 49 & 183 & 291 \\
\hline & SNL/CST 35 & 25 & 45 & 54 \\
\hline & Cyanex TM 272 & 23 & 41 & 58 \\
\hline & SNL/CST 68 & 23 & 38 & 45 \\
\hline & DHDECMP & 22 & 31 & 32 \\
\hline & Duolite ${ }^{\mathrm{TM}} \mathrm{C}-467$ & 19 & 38 & 63 \\
\hline & Amberlyst ${ }^{\mathrm{TM}} 15$ & 15 & 30 & 58 \\
\hline & Duolite $^{\text {TM }}$ CS-100 & 12 & 19 & 25 \\
\hline & Reillex TM HPQ & 8.4 & 8.9 & 8.9 \\
\hline & SRS resorcinol resin & 7.5 & 13 & 22 \\
\hline & Bone char & 6.1 & 9.6 & 13 \\
\hline & Sr-Spec TM & 4.8 & 4.9 & 5.2 \\
\hline
\end{tabular}


Table 16. Uranium Distribution Data (Cont.)

Kd Value for Specified Time

\begin{tabular}{llrrr} 
& Absorber & $\mathbf{3 0} \mathbf{~ m i n}$ & $\mathbf{2 ~ h}$ & $\mathbf{6 h}$ \\
\hline Alkaline & TiO-PAN & 2350 & $>6 \mathrm{~K}$ & $>9 \mathrm{~K}$ \\
Supernate & SNL/HTO & 2127 & $>9 \mathrm{~K}$ & $>17 \mathrm{~K}$ \\
& Diphonix & 124 & 115 & 87 \\
& LANL-TiP & 80 & 51 & 79 \\
& SNL/CST 120 & 73 & 77 & 101 \\
& NaTiO-PAN & 48 & 183 & 403 \\
& SNL/CST 35 & 31 & 56 & 72 \\
& TiFC-PSF & 25 & 93 & 135 \\
& Ionsiv TM TIE-96 & 22 & 77 & 204 \\
& Bone char & 18 & 24 & 28 \\
& SNL/CST 111 & 16 & 30 & 43 \\
& IonsivTM TIE-96 (mod.) & 16 & 64 & 195 \\
& CoFC-PSF & 7.6 & 36 & 92 \\
\hline \hline
\end{tabular}


13. Plutonium. Apart from the outstanding performance of the experimental JSK resins for all contact periods and Sybron $^{\mathrm{TM}}(\mathrm{Et})_{3} \mathrm{~N}$ resin for a 6-h contact period, Reillex ${ }^{\mathrm{TM}}$ HPQ sorbs plutonium from acid-dissolved sludge solution better than all other partitioning agents. The performance of JSK-3 resin, which greatly exceeds that of Reillex ${ }^{\mathrm{TM}} \mathrm{HPQ}$, is particularly impressive.
The poor removal of plutonium from acidified supernate solution may not be a serious problem because the solubility of plutonium is expected to decrease sharply as the acidity decreases. Sorption was not measured from alkaline supernate solution, in which plutonium solubility is expected to be very low.

\begin{tabular}{|c|c|c|c|c|}
\hline \multirow[b]{2}{*}{ Solution } & \multirow[b]{2}{*}{ Absorber } & \multicolumn{3}{|c|}{ Kd Value for Specified Time } \\
\hline & & $30 \mathrm{~min}$ & $2 \mathrm{~h}$ & $6 \mathrm{~h}$ \\
\hline Acid-Dissolved & JSK-3 resin & 1053 & 1035 & 1173 \\
\hline \multirow[t]{11}{*}{ Sludge, $3 \mathrm{~g} \mathrm{Pu} / \mathrm{L}$} & JSK-2 resin & 142 & 278 & 422 \\
\hline & JSK-1 resin & 83 & 143 & 196 \\
\hline & Reillex TM HPQ & 78 & 274 & 485 \\
\hline & TRU-Spec TM & 57 & 58 & 60 \\
\hline & Sybron $^{\mathrm{TM}}(\mathrm{Et})_{3} \mathrm{~N}$ resin & 24 & 114 & 839 \\
\hline & Ionac $^{\mathrm{TM}}$ SR-3 & 21 & 64 & 207 \\
\hline & $\mathrm{Sr}-\mathrm{Spec} \mathrm{TM}^{\mathrm{M}}$ & 14 & 14 & 14 \\
\hline & Purolite $^{\mathrm{TM}}$ A-520-E & 13 & 51 & 415 \\
\hline & DHDECMP & 4.4 & 8.2 & 15 \\
\hline & Duolite $^{\mathrm{TM}}$ CS- 100 & 4.0 & 6.7 & 10 \\
\hline & Cyanex ${ }^{\mathrm{TM}} 923$ & 3.0 & 7.6 & 21 \\
\hline Acid-Dissolved & JSK-3 resin & 870 & 1650 & 2185 \\
\hline \multirow[t]{5}{*}{ Sludge, $60 \mathrm{mg} \mathrm{Pu} / \mathrm{L}$} & JSK-2 resin & 329 & 337 & 261 \\
\hline & JSK-1 resin & 178 & 249 & 254 \\
\hline & Reillex $^{\mathrm{TM}} \mathrm{HPQ}$ & 132 & 184 & 205 \\
\hline & Purolite ${ }^{\mathrm{TM}} \mathrm{A}-520-\mathrm{E}$ & 45 & 186 & 260 \\
\hline & Cyanex ${ }^{\text {TM }} 923$ & 21 & 100 & 199 \\
\hline Acidified & MnO-PAN & 2.8 & 3.3 & 3.8 \\
\hline \multirow[t]{8}{*}{ Supernate } & Duolite $^{\text {TM }}$ CS-100 & 2.7 & 3.4 & 4.4 \\
\hline & Amberlyst ${ }^{\mathrm{TM}} 15$ & 2.3 & 4.9 & 8.5 \\
\hline & LIX TM-54 $^{\text {TM }}$ & 2.2 & 2.8 & 3.0 \\
\hline & Cyanex ${ }^{\mathrm{TM}} 923$ & 1.4 & 1.9 & 2.0 \\
\hline & TRU-Spec TM & 1.2 & 2.0 & 2.8 \\
\hline & Diphonix ${ }^{\mathrm{TM}}$ & 1.0 & 1.2 & 1.0 \\
\hline & ReillexTM HPQ & 0.7 & 0.9 & 0.7 \\
\hline & DHDECMP-DIPB & 0.7 & 0.6 & 0.4 \\
\hline
\end{tabular}

14. Americium. Americium is sorbed from aciddissolved sludge solution by the JSK resins and by Reillex ${ }^{\mathrm{TM}}$ $\mathrm{HPQ}$ but only with single-digit Kd values.

For sorption from acidified supernate solution, many absorbers offer high Kd values for americium. Cyanex ${ }^{\mathrm{TM}} 923$ sorbs americium better than any other partitioning agent studied, including specialty products costing much more.
For sorption from alkaline supernate solution, several absorbers offer triple-digit Kd values for americium. Bone char is particularly effective at the two longer contact times. 
Table 18. Americium Distribution Data

Kd Value for Specified Time

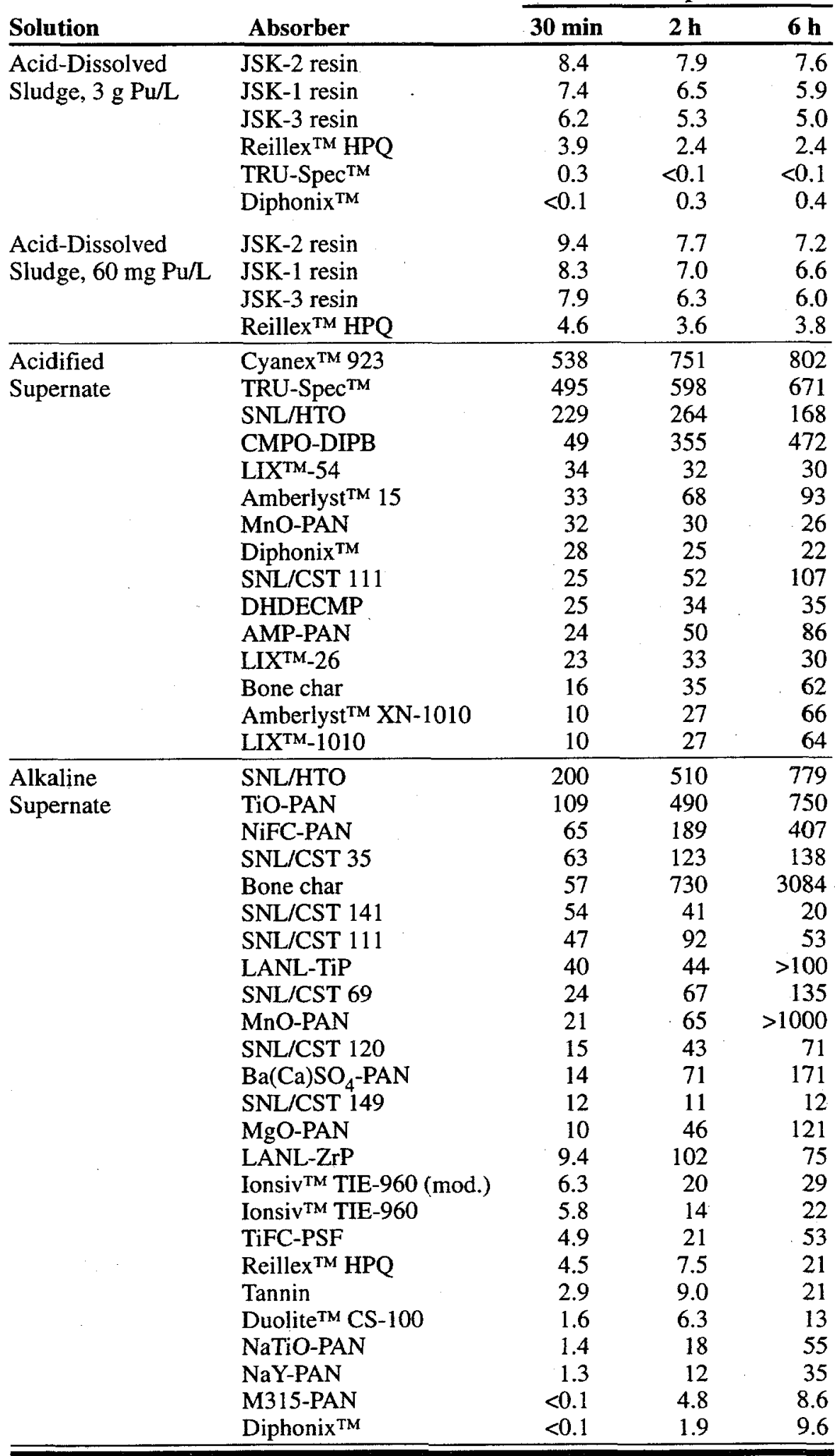




\section{B. Individual Absorbers}

Tables 19 through 81 present $\mathrm{Kd}$ values for sorption of each of the 14 elements onto each of the 63 absorbers from simulated acid-dissolved sludge, acidified supernate, and alkaline supernate solutions. As mentioned earlier, not all absorbers were tested with all three simulant solutions.

Because we measured the sorption of 14 different elements, these tables provide information about unwanted elements most likely to interfere with the target element by competing for absorber sites. Tables 19 through 81 , therefore, may be used to predict the selectivity of each absorber for any element.

Although long-term stability is an essential attribute of any absorber that would be seriously considered for waste processing, we made no attempt to evaluate the stability of these absorbers in the three simulant solutions. The absorbers were tested for sorption from all three simulant solutions unless there was an obvious reason not to, such as apparent degradation of the absorber. We recommend that the absorbers that appear most promising for application to HLW tank processing be evaluated for long-term chemical stability and also for radiation resistance if these properties are not already known.
1. Commercially Available Absorbers. Eighteen commercially available absorbers, listed in Table 4, were included in our study.

\section{a. Amberlyst ${ }^{\mathrm{TM}} 15$ Cation Exchange Resin.} Amberlyst ${ }^{\mathrm{TM}}$ 15, manufactured by Rohm \& Haas, Philadelphia, PA, is a macroporous, strong-acid cation exchange resin with sulfonic acid functional groups. Airdrying the as-received resin before use resulted in a weight loss of $45.4 \%$.

Table 19 shows that surprisingly few elements sorb from acid-dissolved sludge solution onto this resin and that lanthanides and trivalent actinides sorb reasonably well from acidified supernate solution. Amberlyst ${ }^{\mathrm{TM}} 15$ is outperformed by only one other absorber for sorbing strontium from acidified supernate solution. From alkaline supernate solution, cesium, strontium, yttrium, iron, manganese, zinc, and zirconium. are weakly to moderately sorbed. 


\begin{tabular}{|c|c|c|c|c|}
\hline \multirow[b]{2}{*}{ Solution } & \multirow[b]{2}{*}{ Element } & \multicolumn{3}{|c|}{ Kd Value for Specified Time } \\
\hline & & $30 \mathrm{~min}$ & $2 \mathrm{~h}$ & $6 \mathrm{~h}$ \\
\hline $\begin{array}{l}\text { Acid-Dissolved } \\
\text { Sludge }\end{array}$ & $\begin{array}{l}\mathrm{Ce} \\
\mathrm{Cs} \\
\mathrm{Sr} \\
\mathrm{Tc} \\
\mathrm{Y} \\
\mathrm{Cr} \\
\mathrm{Co} \\
\mathrm{Fe} \\
\mathrm{Mn} \\
\mathrm{Zn} \\
\mathrm{Zr} \\
\mathrm{U} \\
\mathrm{Pu} \\
\mathrm{Am}\end{array}$ & $\begin{array}{r}2.0 \\
1.5 \\
1.2 \\
1.0 \\
2.3 \\
1.0 \\
0.9 \\
0.5 \\
0.3 \\
<0.1 \\
1.2 \\
3.1 \\
1.1 \\
0.7\end{array}$ & $\begin{array}{r}1.7 \\
1.3 \\
0.9 \\
0.7 \\
1.7 \\
0.7 \\
0.6 \\
1.2 \\
0.6 \\
<0.1 \\
1.3 \\
6.4 \\
1.9 \\
0.7 \\
\end{array}$ & $\begin{array}{r}1.8 \\
1.6 \\
1.2 \\
1.2 \\
2.1 \\
0.9 \\
0.6 \\
0.8 \\
0.4 \\
<0.1 \\
1.3 \\
7.2 \\
1.8 \\
0.6 \\
\end{array}$ \\
\hline $\begin{array}{l}\text { Acidified } \\
\text { Supernate }\end{array}$ & $\begin{array}{l}\mathrm{Ce} \\
\mathrm{Cs} \\
\mathrm{Sr} \\
\mathrm{Tc} \\
\mathrm{Y} \\
\mathrm{Cr} \\
\mathrm{Co} \\
\mathrm{Fe} \\
\mathrm{Mn} \\
\mathrm{Zn} \\
\mathrm{Zr} \\
\mathrm{U} \\
\mathrm{Pu} \\
\mathrm{Am}\end{array}$ & $\begin{array}{r}42 \\
9.7 \\
12 \\
<0.1 \\
27 \\
4.2 \\
3.6 \\
1.2 \\
5.2 \\
2.4 \\
2.8 \\
15 \\
2.3 \\
33 \\
\end{array}$ & $\begin{array}{r}77 \\
9.1 \\
13 \\
<0.1 \\
45 \\
7.9 \\
3.5 \\
3.4 \\
4.7 \\
2.0 \\
4.6 \\
30 \\
4.9 \\
68 \\
\end{array}$ & $\begin{array}{r}132 \\
9.0 \\
13 \\
<0.1 \\
66 \\
14 \\
3.1 \\
5.8 \\
4.5 \\
1.8 \\
8.4 \\
58 \\
8.5 \\
93 \\
\end{array}$ \\
\hline $\begin{array}{l}\text { Alkaline } \\
\text { Supernate }\end{array}$ & $\begin{array}{l}\mathrm{Ce} \\
\mathrm{Cs} \\
\mathrm{Sr} \\
\mathrm{Tc} \\
\mathrm{Y} \\
\mathrm{Cr} \\
\mathrm{Co} \\
\mathrm{Fe} \\
\mathrm{Mn} \\
\mathrm{Zn} \\
\mathrm{Zr} \\
\mathrm{U} \\
\mathrm{Am}\end{array}$ & $\begin{array}{r}<0.1 \\
7.0 \\
10 \\
0.7 \\
3.1 \\
0.7 \\
1.2 \\
8.2 \\
8.3 \\
5.1 \\
3.6 \\
<0.1 \\
<0.1\end{array}$ & $\begin{array}{r}0.2 \\
6.5 \\
14 \\
0.3 \\
7.0 \\
0.3 \\
1.1 \\
14 \\
13 \\
6.9 \\
6.3 \\
0.2 \\
<0.1\end{array}$ & $\begin{array}{r}0.5 \\
6.4 \\
16 \\
0.4 \\
14 \\
0.4 \\
1.4 \\
22 \\
21 \\
8.2 \\
11 \\
0.3 \\
2.5\end{array}$ \\
\hline
\end{tabular}


b. Amberlyst ${ }^{\mathrm{TM}}$ XN-1010 Cation Exchange Resin. Amberlyst ${ }^{\mathrm{TM}} \mathrm{XN}-1010$, a macroporous, strong-acid cation exchange resin with sulfonic acid functional groups, is manufactured by Rohm \& Haas, Philadelphia, PA. The manufacturer states that Amberlyst ${ }^{\mathrm{TM}} \mathrm{XN}-1010$ has a particularly high surface area and is suitable for nonaqueous applications. Air-drying the as-received resin before use resulted in a weight loss of $4.4 \%$.

Table 20 shows that the behavior of Amberlyst ${ }^{\mathrm{TM}}$ $\mathrm{XN}-1010$ in all three solutions is generally similar to that of Amberlyst ${ }^{\mathrm{TM}} 15$, as would be expected.

Table 20. Amberlyst ${ }^{\mathrm{TM}}$ XN-1010 Cation Exchange Resin: Distribution of 14 Elements from Three Simulant Solutions for Hanford Tank 102-SY

\begin{tabular}{|c|c|c|c|c|}
\hline \multirow[b]{2}{*}{ Solution } & \multirow[b]{2}{*}{ Element } & \multicolumn{3}{|c|}{ Kd Value for Specified Time } \\
\hline & & $30 \mathrm{~min}$ & $2 \mathbf{h}$ & $6 \mathrm{~h}$ \\
\hline Acid-Dissolved & $\mathrm{Ce}$ & 1.3 & 1.4 & 1.5 \\
\hline \multirow{13}{*}{ Sludge } & Cs & 2.4 & 2.2 & 2.5 \\
\hline & $\mathrm{Sr}$ & 0.9 & 0.9 & 1.1 \\
\hline & Tc & 1.2 & 1.1 & 1.3 \\
\hline & $\mathrm{Y}$ & 1.4 & 1.4 & 1.5 \\
\hline & $\mathrm{Cr}$ & 0.9 & 0.6 & 1.3 \\
\hline & Co & 1.3 & 0.9 & 1.2 \\
\hline & $\mathrm{Fe}$ & 0.3 & 1.1 & 0.8 \\
\hline & $\mathrm{Mn}$ & 1.1 & 0.5 & 0.4 \\
\hline & $\mathrm{Zn}$ & 0.3 & $<0.1$ & $<0.1$ \\
\hline & $\mathrm{Zr}$ & 1.2 & 1.8 & 3.3 \\
\hline & $\mathrm{U}$ & 1.2 & 2.4 & 4.6 \\
\hline & $\mathrm{Pu}$ & $<0.1$ & 0.7 & 1.3 \\
\hline & Am & 0.2 & 0.4 & 0.2 \\
\hline Acidified & $\mathrm{Ce}$ & 13 & 38 & 114 \\
\hline \multirow{13}{*}{ Supernate } & Cs & 13 & 15 & 15 \\
\hline & $\mathrm{Sr}$ & 5.9 & 7.8 & 8.3 \\
\hline & Tc & 1.3 & 1.0 & 1.0 \\
\hline & $\mathrm{Y}$ & 11 & 26 & 64 \\
\hline & $\mathrm{Cr}$ & 0.5 & 0.8 & 1.3 \\
\hline & Co & 4.0 & 3.5 & 3.4 \\
\hline & $\mathrm{Fe}$ & $<0.1$ & $<0.1$ & $<0.1$ \\
\hline & $\mathbf{M n}$ & 4.3 & 4.9 & 5.0 \\
\hline & $\mathrm{Zn}$ & 3.2 & 3.4 & 3.6 \\
\hline & $\mathrm{Zr}$ & $<0.1$ & $<0.1$ & $<0.1$ \\
\hline & $\mathrm{U}$ & 4.0 & 6.7 & 15 \\
\hline & $\mathrm{Pu}$ & 0.3 & 0.1 & 0.4 \\
\hline & $\mathrm{Am}$ & 10 & 27 & 66 \\
\hline Alkaline & $\mathrm{Ce}$ & 5.0 & 5.4 & 4.7 \\
\hline \multirow[t]{12}{*}{ Supernate } & Cs & 13 & 14 & 15 \\
\hline & $\mathrm{Sr}$ & 10 & 16 & 15 \\
\hline & Tc & 0.3 & 0.3 & 0.4 \\
\hline & $\mathrm{Y}$ & 3.4 & 4.9 & 4.8 \\
\hline & $\mathrm{Cr}$ & $<0.1$ & $<0.1$ & $<0.1$ \\
\hline & Co & $<0.1$ & 0.6 & 1.3 \\
\hline & $\mathrm{Fe}$ & 3.8 & 10 & 25 \\
\hline & $\mathrm{Mn}$ & 2.3 & 1.8 & 3.3 \\
\hline & $\mathrm{Zn}$ & 1.9 & 6.1 & 22 \\
\hline & $\mathrm{Zr}$ & 3.2 & 8.3 & 25 \\
\hline & $\mathbf{U}$ & 0.6 & 1.0 & 2.1 \\
\hline & $\mathrm{Am}$ & 0.4 & 0.1 & 0.1 \\
\hline
\end{tabular}


d. Diphonix ${ }^{\mathrm{TM}}$ Cation Exchange Resin. Diphonix ${ }^{\mathrm{TM}}$, a polyfunctional resin that contains diphosphonic acid, sulfonic acid, and carboxylic acid functional groups, was obtained from EIChroM Industries, Inc., Darien, IL. Airdrying the as-received resin before use resulted in an unusually large weight loss of $72 \%$.
Diphonix ${ }^{\mathrm{TM}}$ resin sorbs none of the 14 elements significantly from acid-dissolved sludge solution, whereas it sorbs uranium, lanthanides, and americium from acidified supernate solution. Strontium, iron, zinc, zirconium, manganese, and uranium are strongly sorbed from alkaline supernate solution.

Table 22. Diphonix ${ }^{\text {TM }}$ Cation Exchange Resin: Distribution of 14 Elements from Three Simulant Solutions for Hanford Tank 102-SY

\begin{tabular}{|c|c|c|c|c|}
\hline \multirow[b]{2}{*}{ Solution } & \multirow[b]{2}{*}{ Element } & \multicolumn{3}{|c|}{ Kd Value for Specified Time } \\
\hline & & $30 \mathrm{~min}$ & $2 \mathrm{~h}$ & $6 \mathrm{~h}$ \\
\hline Acid-Dissolved & $\mathrm{Ce}$ & 1.3 & 1.1 & 1.2 \\
\hline \multirow[t]{13}{*}{ Sludge } & Cs & 1.7 & 1.4 & 1.3 \\
\hline & $\mathrm{Sr}$ & 2.0 & 1.7 & 1.3 \\
\hline & $\mathrm{Tc}$ & 2.0 & 1.6 & 1.8 \\
\hline & $\mathrm{Y}$ & 1.1 & 1.1 & 1.0 \\
\hline & $\mathrm{Cr}$ & 1.1 & 0.6 & 0.8 \\
\hline & Co & 1.4 & 1.7 & 2.1 \\
\hline & $\mathrm{Fe}$ & 0.2 & 0.6 & 0.3 \\
\hline & $\mathrm{Mn}$ & 1.8 & 3.2 & 3.8 \\
\hline & $\mathrm{Zn}$ & 1.2 & 2.1 & 2.1 \\
\hline & $\mathrm{Zr}$ & 1.0 & 0.6 & 1.0 \\
\hline & $\mathrm{U}$ & 0.4 & 1.5 & 2.8 \\
\hline & $\mathbf{P u}$ & $<0.1$ & $<0.1$ & $<0.1$ \\
\hline & $\mathrm{Am}$ & $<0.1$ & 0.3 & 0.4 \\
\hline Acidified & $\mathrm{Ce}$ & 22 & 23 & 22 \\
\hline \multirow{13}{*}{ Supernate } & Cs & 1.5 & 1.0 & 1.1 \\
\hline & $\mathrm{Sr}$ & 0.6 & 0.2 & 0.2 \\
\hline & Tc & 1.0 & 1.0 & 1.4 \\
\hline & $\mathrm{Y}$ & 28 & 31 & 31 \\
\hline & $\mathrm{Cr}$ & 0.9 & 1.7 & 3.1 \\
\hline & Co & 1.1 & 1.2 & 0.9 \\
\hline & $\mathrm{Fe}$ & 2.1 & 2.7 & 3.1 \\
\hline & $\mathrm{Mn}$ & 0.6 & 0.7 & 0.6 \\
\hline & $\mathrm{Zn}$ & 0.4 & $<0.1$ & $<0.1$ \\
\hline & $\mathrm{Zr}$ & $<0.1$ & $<0.1$ & 0.3 \\
\hline & $\mathrm{U}$ & 96 & 153 & 214 \\
\hline & $\mathrm{Pu}$ & 1.0 & 1.2 & 1.0 \\
\hline & Am & 28 & 25 & 22 \\
\hline Alkaline & $\mathrm{Ce}$ & $<0.1$ & 3.9 & 9.7 \\
\hline \multirow{12}{*}{ Supernate } & Cs & 3.0 & 1.6 & 1.7 \\
\hline & $\mathrm{Sr}$ & 546 & 696 & 918 \\
\hline & Tc & 2.4 & 1.1 & 1.2 \\
\hline & $\mathrm{Y}$ & 16 & 20 & 28 \\
\hline & $\mathrm{Cr}$ & 1.4 & 0.4 & 0.3 \\
\hline & Co & 0.9 & 0.2 & 0.4 \\
\hline & $\mathrm{Fe}$ & 865 & 1762 & 2988 \\
\hline & $\mathrm{Mn}$ & 121 & 200 & 296 \\
\hline & $\mathrm{Zn}$ & 792 & 1616 & 2406 \\
\hline & $\mathrm{Zr}$ & 359 & 759 & 927 \\
\hline & $\mathrm{U}$ & 124 & 115 & 87 \\
\hline & $\mathrm{Am}$ & $<0.1$ & 1.9 & 9.6 \\
\hline
\end{tabular}


c. Duolite ${ }^{\mathrm{TM}}$ C-467 Cation Exchange Resin. Duolite ${ }^{\mathrm{TM}}$ C-467, a chelating cation exchange resin composed of a polystyrene/divinylbenzene copolymer with aminophosphonic acid functional groups, is manufactured by Rohm \& Haas, Philadelphia, PA. Air-drying the as-received resin before use resulted in a weight loss of $34.1 \%$.

Table 21 shows moderate sorption of technetium and zirconium from acid-dissolved sludge solution. From acidified supernate solution, technetium, zinc, and uranium sorb reasonably well, whereas cobalt, manganese, americium, and lanthanides sorb moderately. Strontium and zinc sorb exceptionally well from alkaline supernate, and yttrium, manganese, and americium also sorb well. The much-higher sorption of yttrium suggests that this resin may separate yttrium from cerium in alkaline solution.

\begin{tabular}{|c|c|c|c|c|}
\hline \multirow[b]{2}{*}{ Solution } & \multirow[b]{2}{*}{ Element } & \multicolumn{3}{|c|}{ Kd Value for Specified Time } \\
\hline & & $30 \mathrm{~min}$ & $2 \mathrm{~h}$ & $6 \mathrm{~h}$ \\
\hline $\begin{array}{l}\text { Acid-Dissolved } \\
\text { Sludge }\end{array}$ & $\begin{array}{l}\mathrm{Ce} \\
\mathrm{Cs} \\
\mathrm{Sr} \\
\mathrm{Tc} \\
\mathrm{Y} \\
\mathrm{Cr} \\
\mathrm{Co} \\
\mathrm{Fe} \\
\mathrm{Mn} \\
\mathrm{Zn} \\
\mathrm{Zr} \\
\mathrm{U} \\
\mathrm{Pu} \\
\mathrm{Am}\end{array}$ & $\begin{array}{r}2.4 \\
0.9 \\
1.4 \\
12 \\
1.8 \\
1.1 \\
1.3 \\
0.8 \\
1.3 \\
0.9 \\
5.4 \\
3.8 \\
2.4 \\
0.8\end{array}$ & $\begin{array}{r}1.5 \\
0.2 \\
0.6 \\
12 \\
0.9 \\
0.4 \\
0.4 \\
0.2 \\
1.3 \\
0.7 \\
7.7 \\
5.0 \\
3.2 \\
0.7\end{array}$ & $\begin{array}{c}2.2 \\
0.7 \\
0.9 \\
13 \\
1.6 \\
1.0 \\
0.8 \\
0.4 \\
1.4 \\
0.8 \\
13 \\
6.0 \\
3.9 \\
0.7\end{array}$ \\
\hline $\begin{array}{l}\text { Acidified } \\
\text { Supernate }\end{array}$ & $\begin{array}{l}\mathrm{Ce} \\
\mathrm{Cs} \\
\mathrm{Sr} \\
\mathrm{Tc} \\
\mathrm{Y} \\
\mathrm{Cr} \\
\mathrm{Co} \\
\mathrm{Fe} \\
\mathrm{Mn} \\
\mathrm{Zn} \\
\mathrm{Zr} \\
\mathrm{U} \\
\mathrm{Pu} \\
\mathrm{Am}\end{array}$ & $\begin{array}{r}6.3 \\
<0.1 \\
1.4 \\
40 \\
7.2 \\
0.5 \\
14 \\
<0.1 \\
15 \\
24 \\
<0.1 \\
19 \\
0.7 \\
6.9\end{array}$ & $\begin{array}{r}4.2 \\
<0.1 \\
0.7 \\
42 \\
5.6 \\
0.7 \\
13 \\
0.6 \\
17 \\
46 \\
0.1 \\
38 \\
1.0 \\
6.2\end{array}$ & $\begin{array}{c}3.6 \\
0.1 \\
0.5 \\
44 \\
4.5 \\
1.1 \\
12 \\
0.8 \\
17 \\
89 \\
0.3 \\
63 \\
1.3 \\
5.4\end{array}$ \\
\hline $\begin{array}{l}\text { Alkaline } \\
\text { Supernate }\end{array}$ & $\begin{array}{l}\mathrm{Ce} \\
\mathrm{Cs} \\
\mathrm{Sr} \\
\mathrm{Tc} \\
\mathrm{Y} \\
\mathrm{Cr} \\
\mathrm{Co} \\
\mathrm{Fe} \\
\mathrm{Mn} \\
\mathrm{Zn} \\
\mathrm{Zr} \\
\mathrm{U} \\
\mathrm{Am}\end{array}$ & $\begin{array}{r}0.4 \\
0.4 \\
1051 \\
2.5 \\
19 \\
<0.1 \\
1.0 \\
6.6 \\
52 \\
292 \\
1.0 \\
0.2 \\
1.2\end{array}$ & $\begin{array}{r}1.5 \\
0.2 \\
2734 \\
3.7 \\
40 \\
<0.1 \\
1.0 \\
7.0 \\
80 \\
617 \\
1.4 \\
<0.1 \\
7.5\end{array}$ & $\begin{array}{r}2.0 \\
0.2 \\
>5 \mathrm{~K} \\
4.7 \\
85 \\
0.1 \\
1.2 \\
8.0 \\
93 \\
910 \\
1.6 \\
0.3 \\
12\end{array}$ \\
\hline
\end{tabular}


e. Ionsiv TM TIE-96 Absorber. Ionsiv TM TIE-96 is a titanium-loaded zeolite manufactured by UOP Molecular Sieves Division, Moorestown, NJ. This absorber was used as received.
Ionsiv ${ }^{\text {TM }}$ TIE-96 sorbs zirconium well and cesium slightly from acid-dissolved sludge solution. From acidified supernate solution, it sorbs cesium well and uranium somewhat. It sorbs cesium, strontium, iron, manganese, zinc, zirconium, and uranium well from alkaline supernate solution.

Table 23. Ionsiv $^{\mathrm{TM}}$ TIE-96: Distribution of 14 Elements from Three Simulant Solutions for Hanford Tank 102-SY

\begin{tabular}{|c|c|c|c|c|}
\hline \multirow[b]{2}{*}{ Solution } & \multirow[b]{2}{*}{ Element } & \multicolumn{3}{|c|}{ Kd Value for Specified Time } \\
\hline & & $30 \mathrm{~min}$ & $2 \mathrm{~h}$ & $6 \mathrm{~h}$ \\
\hline Acid-Dissolved & $\mathrm{Ce}$ & 1.8 & 1.1 & 1.5 \\
\hline \multirow[t]{13}{*}{ Sludge } & Cs & 7.6 & 7.9 & 8.3 \\
\hline & $\mathrm{Sr}$ & 1.9 & 1.0 & 1.4 \\
\hline & $\mathrm{Tc}$ & 1.7 & 1.6 & 1.5 \\
\hline & $\mathrm{Y}$ & 1.5 & 0.9 & 1.2 \\
\hline & $\mathrm{Cr}$ & 1.4 & 0.8 & 1.1 \\
\hline & $\mathrm{Co}$ & 1.2 & 0.7 & 0.8 \\
\hline & $\mathrm{Fe}$ & 0.7 & 0.4 & 0.8 \\
\hline & $\mathrm{Mn}$ & 0.1 & 0.1 & 0.1 \\
\hline & $\mathrm{Zn}$ & 0.4 & 0.2 & 0.3 \\
\hline & $\mathrm{Zr}$ & 28 & 64 & 85 \\
\hline & $\mathrm{U}$ & $<0.1$ & 0.4 & 0.1 \\
\hline & $\mathrm{Pu}$ & 1.5 & 2.5 & 4.4 \\
\hline & $\mathrm{Am}$ & 0.2 & $<0.1$ & 0.3 \\
\hline Acidified & $\mathrm{Ce}$ & 0.1 & 1.2 & 1.6 \\
\hline \multirow{13}{*}{ Supernate } & Cs & 58 & 97 & 122 \\
\hline & $\mathrm{Sr}$ & 0.5 & 0.7 & 0.4 \\
\hline & Tc & $<0.1$ & $<0.1$ & $<0.1$ \\
\hline & $\mathrm{Y}$ & 0.9 & 1.3 & 1.1 \\
\hline & $\mathrm{Cr}$ & $<0.1$ & 0.9 & 0.9 \\
\hline & $\mathrm{Co}$ & 0.2 & 0.6 & 0.4 \\
\hline & $\mathrm{Fe}$ & 0.5 & 0.4 & 0.5 \\
\hline & $\mathrm{Mn}$ & 0.4 & 0.3 & 0.3 \\
\hline & $\mathrm{Zn}$ & 0.6 & 0.2 & 0.4 \\
\hline & $\mathrm{Zr}$ & $<0.1$ & 0.6 & 0.5 \\
\hline & $\mathrm{U}$ & 5.4 & 7.6 & 12 \\
\hline & $\mathrm{Pu}$ & $<0.1$ & 0.3 & 0.3 \\
\hline & $\mathrm{Am}$ & 1.5 & 1.9. & 2.2 \\
\hline Alkaline & $\mathrm{Ce}$ & 2.3 & 4.4 & 7.1 \\
\hline \multirow[t]{12}{*}{ Supernate } & $\mathrm{Cs}$ & 47 & 84 & 103 \\
\hline & $\mathrm{Sr}$ & 118 & 140 & 159 \\
\hline & Tc & 0.3 & 0.3 & 0.3 \\
\hline & $\mathrm{Y}$ & 16 & 20 & 23 \\
\hline & $\mathrm{Cr}$ & $<0.1$ & 0.3 & 0.4 \\
\hline & Co & 0.9 & 1.6 & 1.9 \\
\hline & $\mathrm{Fe}$ & 166 & 139 & 124 \\
\hline & $\mathrm{Mn}$ & 103 & 115 & 126 \\
\hline & $\mathrm{Zn}$ & 49 & 130 & 227 \\
\hline & $\mathrm{Zr}$ & 117 & 151 & 129 \\
\hline & $\mathrm{U}$ & 22 & 77 & 204 \\
\hline & $\mathrm{Am}$ & 5.8 & 14 & 22 \\
\hline
\end{tabular}


f. Ionsiv ${ }^{\mathrm{TM}}$ TIE-96 (Modified) Absorber. Ionsiv ${ }^{\mathrm{TM}}$ TIE-96 (modified) is an improved version of the Ionsiv TIE-96 titanium-loaded zeolite manufactured by UOP Molecular Sieves Division, Moorestown, NJ. This absorber was used as received.

The modified form of Ionsiv ${ }^{T M}$ TIE-96 provides a generally improved version of the sorption pattern of unmodified IonsivTM TIE-96, with the Kd values of lanthanides, uranium, and americium for sorption from acidified supernate solution approximately doubled.

The sorption pattern from alkaline supernate solution also is similar to that of unmodified Ionsiv ${ }^{\text {TM }}$ TIE96 , but the Kd values generally are higher.

Table 24. Ionsiv ${ }^{\mathrm{TM}}$ TIE-96 (Modified): Distribution of 14 Elements from Three Simulant Solutions for Hanford Tank 102-SY

\begin{tabular}{|c|c|c|c|c|}
\hline \multirow[b]{2}{*}{ Solution } & \multirow[b]{2}{*}{ Element } & \multicolumn{3}{|c|}{ Kd Value for Specified Time } \\
\hline & & $30 \mathrm{~min}$ & $2 \mathrm{~h}$ & $6 \mathrm{~h}$ \\
\hline Acid-Dissolved & $\mathrm{Ce}$ & 0.9 & 1.1 & 1.3 \\
\hline \multirow{13}{*}{ Sludge } & Cs & 7.7 & 9.0 & 9.4 \\
\hline & $\mathrm{Sr}$ & 1.4 & 1.4 & 1.4 \\
\hline & Tc & 1.0 & 1.0 & 1.5 \\
\hline & $Y$ & 1.2 & 1.0 & 1.0 \\
\hline & $\mathrm{Cr}$ & 0.4 & 0.9 & 1.1 \\
\hline & Co & 0.7 & 0.8 & 0.6 \\
\hline & $\mathrm{Fe}$ & 0.2 & 0.7 & 0.6 \\
\hline & $\mathrm{Mn}$ & 0.3 & 0.1 & 0.1 \\
\hline & $\mathrm{Zn}$ & 0.3 & 0.2 & 0.1 \\
\hline & $\mathrm{Zr}$ & 35 & 88 & 115 \\
\hline & $\mathrm{U}$ & $<0.1$ & 0.2 & 0.3 \\
\hline & $\mathrm{Pu}$ & 1.8 & 3.0 & 4.9 \\
\hline & $\mathrm{Am}$ & 0.3 & 0.1 & 0.3 \\
\hline Acidified & $\mathrm{Ce}$ & 2.3 & 3.0 & 2.9 \\
\hline \multirow[t]{13}{*}{ Supernate } & $\mathrm{Cs}$ & 63 & 110 & 139 \\
\hline & $\mathrm{Sr}$ & 0.9 & 1.0 & 0.7 \\
\hline & $\mathrm{Tc}$ & $<0.1$ & $<0.1$ & $<0.1$ \\
\hline & $\mathrm{Y}$ & 2.2 & 2.2 & 1.9 \\
\hline & $\mathrm{Cr}$ & 1.0 & 1.6 & 1.3 \\
\hline & Co & 1.0 & 0.8 & 0.8 \\
\hline & $\mathrm{Fe}$ & 0.5 & 0.6 & 0.8 \\
\hline & $\mathrm{Mn}$ & 0.3 & 0.4 & 0.5 \\
\hline & $\mathrm{Zn}$ & 0.8 & 0.3 & 0.5 \\
\hline & $\mathrm{Zr}$ & 0.6 & 0.8 & 0.6 \\
\hline & $\mathrm{U}$ & 7.3 & 14 & 26 \\
\hline & $\mathrm{Pu}$ & $<0.1$ & 0.4 & 0.2 \\
\hline & $\mathrm{Am}$ & 4.6 & 5.6 & 5.7 \\
\hline Alkaline & $\mathrm{Ce}$ & 2.6 & 5.9 & 11 \\
\hline \multirow[t]{12}{*}{ Supernate } & Cs & 49 & 86 & 107 \\
\hline & $\mathrm{Sr}$ & 286 & 405 & 421 \\
\hline & $\mathrm{Tc}$ & 0.2 & 0.2 & 0.2 \\
\hline & $\mathrm{Y}$ & 28 & 41 & 47 \\
\hline & $\mathrm{Cr}$ & $<0.1$ & 0.2 & 0.3 \\
\hline & Co & 1.2 & 1.6 & 2.1 \\
\hline & $\mathrm{Fe}$ & 216 & 515 & 430 \\
\hline & $\mathrm{Mn}$ & 121 & 280 & 322 \\
\hline & $\mathrm{Zn}$ & 29 & 96 & 198 \\
\hline & $\mathrm{Zr}$ & 146 & 379 & 327 \\
\hline & $\mathrm{U}$ & 16 & 64 & 195 \\
\hline & $\mathrm{Am}$ & 6.3 & 20 & 29 \\
\hline
\end{tabular}


g. Duolite ${ }^{\mathrm{TM}}$ CS-100 Resin. Duolite ${ }^{\mathrm{TM}} \mathrm{CS}-100$ is a carboxy methylated phenol formaldehyde polymer manufactured by Rohm \& Haas, Philadelphia, PA. Air-drying the as-received resin before use resulted in a weight loss of $55 \%$.

Duolite ${ }^{\text {TM }}$ CS-100 resin moderately sorbs zirconium, plutonium, and technetium (but not cesium) from acid-dissolved sludge solution. Only uranium is sorbed to a useful extent from acidified supernate solution.

Zinc, zirconium, cesium, and iron are strongly sorbed from alkaline supernate solution. During contact with this resin, the alkaline supernate solution turned brown, indicating possible resin instability.

Table 25. Duolite ${ }^{\mathrm{TM}}$ CS-100 Cation Exchange Resin: Distribution of 14 Elements from Three Simulant Solutions for Hanford Tank 102-SY

\begin{tabular}{|c|c|c|c|c|}
\hline \multirow[b]{2}{*}{ Solution } & \multirow[b]{2}{*}{ Element } & \multicolumn{3}{|c|}{ Kd Value for Specified Time } \\
\hline & & $30 \mathrm{~min}$ & $2 h$ & $6 \mathrm{~h}$ \\
\hline Acid-Dissolved & $\mathrm{Ce}$ & 0.2 & 0.5 & 0.5 \\
\hline \multirow{13}{*}{ Sludge } & $\mathrm{Cs}$ & 0.5 & 1.1 & 1.3 \\
\hline & $\mathrm{Sr}$ & $<0.1$ & $<0.1$ & $<0.1$ \\
\hline & $\mathrm{Tc}$ & 4.8 & 6.3 & 6.4 \\
\hline & $\mathrm{Y}$ & 0.2 & 0.3 & 0.2 \\
\hline & $\mathrm{Cr}$ & 0.1 & 0.1 & 0.2 \\
\hline & $\mathrm{Co}$ & 0.3 & 0.5 & 0.4 \\
\hline & $\mathrm{Fe}$ & 0.8 & 0.8 & 1.4 \\
\hline & $\mathrm{Mn}$ & 0.1 & 0.1 & 0.3 \\
\hline & $\mathrm{Zn}$ & $<0.1$ & $<0.1$ & 0.2 \\
\hline & $\mathrm{Zr}$ & 9.2 & 16 & 22 \\
\hline & $\mathbf{U}$ & 1.2 & 1.6 & 2.4 \\
\hline & $\mathrm{Pu}$ & 4.0 & 6.7 & 10 \\
\hline & Am & 0.1 & $<0.1$ & $<0.1$ \\
\hline Acidified & $\mathrm{Ce}$ & 2.6 & 2.5 & 3.0 \\
\hline \multirow{13}{*}{ Supernate } & $\mathrm{Cs}$ & 1.3 & 1.2 & 1.3 \\
\hline & $\mathrm{Sr}$ & $<0.1$ & $<0.1$ & $<0.1$ \\
\hline & Tc & 2.2 & 2.6 & 2.8 \\
\hline & $\mathrm{Y}$ & 2.4 & 2.4 & 2.4 \\
\hline & $\mathrm{Cr}$ & 1.0 & 2.3 & 3.5 \\
\hline & Co & 0.5 & 0.4 & 0.5 \\
\hline & $\mathrm{Fe}$ & 1.8 & 2.6 & 3.2 \\
\hline & $\mathrm{Mn}$ & $<0.1$ & $<0.1$ & $<0.1$ \\
\hline & $\mathrm{Zn}$ & 1.1 & 0.9 & 0.8 \\
\hline & $\mathrm{Zr}$ & 1.1 & 2.1 & 3.1 \\
\hline & $\mathrm{U}$ & 12 & 19 & 25 \\
\hline & $\mathrm{Pu}$ & 2.7 & 3.4 & 4.4 \\
\hline & $\mathrm{Am}$ & 3.1 & 3.0 & 2.9 \\
\hline Alkaline & $\mathrm{Ce}$ & 3.7 & 4.0 & 5.4 \\
\hline \multirow[t]{12}{*}{ Supernate } & $\mathrm{Cs}$ & 196 & 225 & 233 \\
\hline & $\mathrm{Sr}$ & 26 & 29 & 40 \\
\hline & $\mathrm{Tc}$ & $<0.1$ & $<0.1$ & $<0.1$ \\
\hline & $\mathrm{Y}$ & 10 & 14 & 21 \\
\hline & $\mathrm{Cr}$ & 0.3 & 0.4 & 0.6 \\
\hline & $\mathrm{Co}$ & 8.1 & 12 & 16 \\
\hline & $\mathrm{Fe}$ & 52 & 75 & 110 \\
\hline & $\mathrm{Mn}$ & 4.8 & 9.7 & 18 \\
\hline & $\mathrm{Zn}$ & 122 & 286 & 490 \\
\hline & $\mathrm{Zr}$ & 142 & 222 & 304 \\
\hline & $\mathrm{U}$ & 15 & 14 & 18 \\
\hline & $\mathrm{Am}$ & 1.6 & 6.3 & 13 \\
\hline
\end{tabular}


h. Tannin Absorber. Tannin absorber was obtained from Mitsubishi Nuclear Fuels Corporation, Japan. Airdrying the as-received tannin resulted in an unusually high weight loss of $82.2 \%$.

Tannin sorbs technetium and zirconium from aciddissolved sludge solution, although the sorption kinetics are slow. Only uranium is sorbed from acidified supernate solution with a $\mathrm{Kd}$ value higher than 5 , and again the sorption kinetics appear slow. Zirconium, zinc, iron, strontium, and cesium sorb well from alkaline supernate solution.

Table 26. Tannin: Distribution of 14 Elements from Three Simulant Solutions for Hanford Tank 102-SY

\begin{tabular}{|c|c|c|c|c|}
\hline \multirow[b]{2}{*}{ Solution } & \multirow[b]{2}{*}{ Element } & \multicolumn{3}{|c|}{ Kd Value for Specified Time } \\
\hline & & $30 \mathrm{~min}$ & $\mathbf{2 h}$ & $6 \mathrm{~h}$ \\
\hline $\begin{array}{l}\text { Acid-Dissolved } \\
\text { Sludge }\end{array}$ & $\begin{array}{l}\mathrm{Ce} \\
\mathrm{Cs} \\
\mathrm{Sr} \\
\mathrm{Tc} \\
\mathrm{Y} \\
\mathrm{Cr} \\
\mathrm{Co} \\
\mathrm{Fe} \\
\mathrm{Mn} \\
\mathrm{Zn} \\
\mathrm{Zr} \\
\mathrm{U} \\
\mathrm{Pu} \\
\mathrm{Am}\end{array}$ & $\begin{array}{r}1.0 \\
1.8 \\
0.9 \\
10 \\
1.2 \\
0.6 \\
1.3 \\
0.3 \\
0.2 \\
0.4 \\
4.3 \\
<0.1 \\
<0.1 \\
0.2 \\
\end{array}$ & $\begin{array}{c}0.6 \\
1.5 \\
0.6 \\
39 \\
1.2 \\
0.6 \\
3.4 \\
0.2 \\
0.3 \\
0.3 \\
12 \\
0.6 \\
0.6 \\
0.1\end{array}$ & $\begin{array}{r}0.5 \\
1.3 \\
0.3 \\
136 \\
0.7 \\
0.3 \\
13 \\
0.7 \\
0.2 \\
0.2 \\
33 \\
1.0 \\
2.5 \\
0.2 \\
\end{array}$ \\
\hline $\begin{array}{l}\text { Acidified } \\
\text { Supernate }\end{array}$ & $\begin{array}{l}\mathrm{Ce} \\
\mathrm{Cs} \\
\mathrm{Sr} \\
\mathrm{Tc} \\
\mathrm{Y} \\
\mathrm{Cr} \\
\mathrm{Co} \\
\mathrm{Fe} \\
\mathrm{Mn} \\
\mathrm{Zn} \\
\mathrm{Zr} \\
\mathrm{U} \\
\mathrm{Pu} \\
\mathrm{Am}\end{array}$ & $\begin{array}{r}1.9 \\
2.4 \\
1.0 \\
2.1 \\
1.8 \\
0.1 \\
1.6 \\
<0.1 \\
1.3 \\
0.6 \\
<0.1 \\
2.2 \\
0.4 \\
1.1\end{array}$ & $\begin{array}{r}3.7 \\
2.2 \\
0.9 \\
2.7 \\
3.3 \\
0.3 \\
1.6 \\
<0.1 \\
0.4 \\
0.2 \\
<0.1 \\
4.2 \\
0.2 \\
2.0\end{array}$ & $\begin{array}{r}4.1 \\
2.4 \\
0.7 \\
2.7 \\
4.1 \\
0.6 \\
1.0 \\
0.2 \\
0.5 \\
0.2 \\
<0.1 \\
8.8 \\
0.4 \\
3.1 \\
\end{array}$ \\
\hline $\begin{array}{l}\text { Alkaline } \\
\text { Supernate }\end{array}$ & $\begin{array}{l}\mathrm{Ce} \\
\mathrm{Cs} \\
\mathrm{Sr} \\
\mathrm{Tc} \\
\mathrm{Y} \\
\mathrm{Cr} \\
\mathrm{Co} \\
\mathrm{Fe} \\
\mathrm{Mn} \\
\mathrm{Zn} \\
\mathrm{Zr} \\
\mathrm{U} \\
\mathrm{Am}\end{array}$ & $\begin{array}{r}9.4 \\
64 \\
50 \\
4.3 \\
12 \\
1.9 \\
<0.1 \\
56 \\
7.1 \\
60 \\
131 \\
9.1 \\
2.9\end{array}$ & $\begin{array}{r}13 \\
66 \\
58 \\
5.4 \\
18 \\
7.3 \\
1.1 \\
90 \\
16 \\
143 \\
231 \\
24 \\
9.0\end{array}$ & $\begin{array}{r}22 \\
66 \\
102 \\
4.0 \\
34 \\
27 \\
2.5 \\
160 \\
34 \\
251 \\
372 \\
46 \\
21\end{array}$ \\
\hline
\end{tabular}


i. Sr-Spec ${ }^{\mathrm{TM}}$ Resin. Sr-Spec ${ }^{\mathrm{TM}}$ resin, a crown-etherbased material, was obtained from EIChroM Industries, Inc., Darien, IL. This resin was used as received.

The Kd value of only 3 for sorbing strontium from acid-dissolved sludge is nearly matched by the $\mathrm{Kd}$ value of 2.8 for technetium and is exceeded by the $\mathrm{Kd}$ values for uranium and plutonium.
Technetium, strontium, and uranium sorb from acidified supernate solution. Solution discoloration, observed in acidified supernate solution, indicated that Sr-Spec ${ }^{\mathrm{TM}}$ may be somewhat unstable in that medium. Only technetium sorbs well from alkaline supernate solution.

Table 27. Sr-Spec ${ }^{T M}$ Resin: Distribution of 14 Elements from Three Simulant Solutions for Hanford Tank 102-SY

\begin{tabular}{|c|c|c|c|c|}
\hline \multirow[b]{2}{*}{ Solution } & \multirow[b]{2}{*}{ Element } & \multicolumn{3}{|c|}{ Kd Value for Specified Time } \\
\hline & & $30 \mathrm{~min}$ & $2 \mathrm{~h}$ & $6 \mathrm{~h}$ \\
\hline Acid-Dissolved & $\mathrm{Ce}$ & 0.3 & 0.1 & 0.3 \\
\hline \multirow[t]{13}{*}{ Sludge } & Cs & 0.3 & 0.1 & 0.1 \\
\hline & $\mathrm{Sr}$ & 3.0 & 3.1 & 2.9 \\
\hline & $\mathrm{Tc}$ & 2.8 & 2.7 & 2.8 \\
\hline & $Y$ & 0.6 & 0.4 & 0.5 \\
\hline & $\mathrm{Cr}$ & $<0.1$ & 0.1 & 0.2 \\
\hline & $\mathrm{Co}$ & 0.4 & 0.1 & 0.2 \\
\hline & $\mathrm{Fe}$ & $<0.1$ & 0.2 & 0.2 \\
\hline & $\mathrm{Mn}$ & 0.2 & 0.1 & 0.3 \\
\hline & $\mathrm{Zn}$ & 0.1 & 0.2 & 0.2 \\
\hline & $\mathrm{Zr}$ & 1.1 & 1.5 & 1.6 \\
\hline & $\mathbf{U}$ & 4.9 & 5.1 & 5.7 \\
\hline & $\mathrm{Pu}$ & 14 & 14 & 14 \\
\hline & $\mathrm{Am}$ & 0.1 & 0.2 & 0.1 \\
\hline Acidified & $\mathrm{Ce}$ & 1.0 & 0.8 & 0.8 \\
\hline \multirow{13}{*}{ Supernate } & $\mathrm{Cs}$ & 0.9 & 0.9 & 0.9 \\
\hline & $\mathrm{Sr}$ & 18 & 18 & 17 \\
\hline & $\mathrm{Tc}$ & 45 & 42 & 39 \\
\hline & $\mathrm{Y}$ & $<0.1$ & $<0.1$ & $<0.1$ \\
\hline & $\mathrm{Cr}$ & 1.3 & 1.6 & 2.0 \\
\hline & $\mathrm{Co}$ & 1.1 & 0.8 & 0.8 \\
\hline & $\mathrm{Fe}$ & 0.3 & 0.8 & 1.5 \\
\hline & $\mathrm{Mn}$ & 0.1 & 0.3 & 0.4 \\
\hline & $\mathrm{Zn}$ & $<0.1$ & $<0.1$ & $<0.1$ \\
\hline & $\mathrm{Zr}$ & 1.6 & 1.8 & 2.1 \\
\hline & $\mathrm{U}$ & 4.8 & 4.9 & 5.2 \\
\hline & $\mathrm{Pu}$ & 0.4 & 0.7 & 1.0 \\
\hline & Am & 0.1 & 0.5 & 0.3 \\
\hline Alkaline & $\mathrm{Ce}$ & 6.4 & 7.6 & 5.7 \\
\hline \multirow[t]{12}{*}{ Supernate } & Cs & 0.2 & $<0.1$ & 0.1 \\
\hline & $\mathrm{Sr}$ & 11 & 11 & 9.3 \\
\hline & $\mathrm{Tc}$ & 82 & 81 & 76 \\
\hline & $\mathbf{Y}$ & 7.9 & 8.5 & 6.7 \\
\hline & $\mathrm{Cr}$ & 0.3 & 0.3 & 0.6 \\
\hline & $\mathrm{Co}$ & 0.3 & $<0.1$ & 0.2 \\
\hline & $\mathrm{Fe}$ & 0.5 & 0.9 & 2.6 \\
\hline & $\mathrm{Mn}$ & $<0.1$ & 0.9 & 4.3 \\
\hline & $\mathrm{Zn}$ & $<0.1$ & $<0.1$ & 0.1 \\
\hline & $\mathrm{Zr}$ & 1.4 & 1.5 & 1.9 \\
\hline & $\mathbf{U}$ & $<0.1$ & 0.5 & 0.6 \\
\hline & $\mathrm{Am}$ & $<0.1$ & $<0.1$ & 3.3 \\
\hline
\end{tabular}


j. TRU-Spec ${ }^{\mathrm{TM}}$ Resin. TRU-Spec ${ }^{\mathrm{TM}}$ resin, a mixture of 13\% CMPO (octylphenyl-N, N-diisobutylcarbamoylmethylphosphine oxide) and 27\% TBP (tributyl phosphate) sorbed on polyacrylic ester beads, was obtained from EIChroM Industries, Inc., Darien, IL. This absorber was used as received.
This resin sorbs plutonium and uranium reasonably well from acid-dissolved sludge, whereas it sorbs technetium and zirconium weakly. It sorbs lanthanides, americium, uranium, and technetium well from acidified supernate solution. No elements sorb on this resin with even double-digit $\mathrm{Kd}$ values from alkaline supernate solution.

\begin{tabular}{|c|c|c|c|c|}
\hline \multirow[b]{2}{*}{ Solution } & \multirow[b]{2}{*}{ Element } & \multicolumn{3}{|c|}{ Kd Value for Specified Time } \\
\hline & & $30 \mathrm{~min}$ & $2 \mathbf{h}$ & $6 \mathrm{~h}$ \\
\hline $\begin{array}{l}\text { Acid-Dissolved } \\
\text { Sludge }\end{array}$ & $\begin{array}{l}\mathrm{Ce} \\
\mathrm{Cs} \\
\mathrm{Sr} \\
\mathrm{Tc} \\
\mathrm{Y} \\
\mathrm{Cr} \\
\mathrm{Co} \\
\mathrm{Fe} \\
\mathrm{Mn} \\
\mathrm{Zn} \\
\mathrm{Zr} \\
\mathrm{U} \\
\mathrm{Pu} \\
\mathrm{Am}\end{array}$ & $\begin{array}{r}0.1 \\
0.2 \\
0.2 \\
4.1 \\
0.1 \\
0.6 \\
<0.1 \\
0.8 \\
<0.1 \\
0.6 \\
3.8 \\
25 \\
57 \\
0.3\end{array}$ & $\begin{array}{r}0.2 \\
0.3 \\
0.2 \\
4.0 \\
0.3 \\
0.6 \\
<0.1 \\
0.8 \\
0.1 \\
0.5 \\
4.3 \\
26 \\
58 \\
0.1\end{array}$ & $\begin{array}{r}0.4 \\
0.3 \\
0.3 \\
4.1 \\
0.4 \\
0.8 \\
<0.1 \\
0.8 \\
0.4 \\
0.6 \\
4.9 \\
27 \\
60 \\
0.1\end{array}$ \\
\hline $\begin{array}{l}\text { Acidified } \\
\text { Supernate }\end{array}$ & $\begin{array}{l}\mathrm{Ce} \\
\mathrm{Cs} \\
\mathrm{Sr} \\
\mathrm{Tc} \\
\mathrm{Y} \\
\mathrm{Cr} \\
\mathrm{Co} \\
\mathrm{Fe} \\
\mathrm{Mn} \\
\mathrm{Zn} \\
\mathrm{Zr} \\
\mathrm{U} \\
\mathrm{Pu} \\
\mathrm{Am} \\
\end{array}$ & $\begin{array}{r}548 \\
1.5 \\
0.5 \\
90 \\
98 \\
1.3 \\
<0.1 \\
0.8 \\
0.3 \\
<0.1 \\
1.4 \\
70 \\
1.2 \\
495 \\
\end{array}$ & $\begin{array}{r}594 \\
1.8 \\
0.2 \\
78 \\
94 \\
1.9 \\
<0.1 \\
1.4 \\
0.3 \\
<0.1 \\
1.9 \\
107 \\
2.0 \\
598 \\
\end{array}$ & $\begin{array}{r}644 \\
2.0 \\
0.1 \\
74 \\
92 \\
2.5 \\
<0.1 \\
2.5 \\
0.3 \\
<0.1 \\
2.6 \\
164 \\
2.8 \\
671 \\
\end{array}$ \\
\hline $\begin{array}{l}\text { Alkaline } \\
\text { Supernate }\end{array}$ & $\begin{array}{l}\mathrm{Ce} \\
\mathrm{Cs} \\
\mathrm{Sr} \\
\mathrm{Tc} \\
\mathrm{Y} \\
\mathrm{Cr} \\
\mathrm{Co} \\
\mathrm{Fe} \\
\mathrm{Mn} \\
\mathrm{Zn} \\
\mathrm{Zr} \\
\mathrm{U} \\
\mathrm{Am}\end{array}$ & $\begin{array}{r}5.9 \\
<0.1 \\
4.9 \\
7.6 \\
5.9 \\
<0.1 \\
0.2 \\
0.4 \\
<0.1 \\
0.3 \\
0.7 \\
<0.1 \\
<0.1\end{array}$ & $\begin{array}{r}4.6 \\
<0.1 \\
4.5 \\
7.5 \\
5.6 \\
0.3 \\
0.2 \\
0.7 \\
0.2 \\
0.2 \\
1.1 \\
0.3 \\
<0.1\end{array}$ & $\begin{array}{r}6.7 \\
<0.1 \\
6.6 \\
7.2 \\
8.2 \\
0.1 \\
0.2 \\
1.7 \\
2.4 \\
0.1 \\
1.2 \\
0.5 \\
0.9\end{array}$ \\
\hline
\end{tabular}


k. Durasil TM 190 Resin. Durasil ${ }^{\mathrm{TM}} 190$ resin, obtained from Duratek Corporation, Beltsville, MD, was used as received. The air-dried weight, per water-wet milliliter, was $0.969 \mathrm{~g}$.
This resin sorbs none of the 14 elements significantly from acid-dissolved sludge solution. It sorbs cesium from acidified supernate solution. Only cerium, strontium, and yttrium approach even low double-digit $\mathrm{Kd}$ values for sorption from alkaline supernate solution.

Table 29. Durasil ${ }^{\mathrm{TM}} 190$ Resin: Distribution of 14 Elements from Three Simulant Solutions for Hanford Tank 102-SY

\begin{tabular}{|c|c|c|c|c|}
\hline \multirow[b]{2}{*}{ Solution } & \multirow[b]{2}{*}{ Element } & \multicolumn{3}{|c|}{ Kd Value for Specified Tim } \\
\hline & & $30 \mathrm{~min}$ & $2 \mathrm{~h}$ & $6 \mathrm{~h}$ \\
\hline Acid-Dissolved & $\mathrm{Ce}$ & 0.4 & 0.5 & 0.7 \\
\hline \multirow{13}{*}{ Sludge } & Cs & 1.2 & 1.7 & 2.5 \\
\hline & $\mathrm{Sr}$ & 0.1 & $<0.1$ & 0.3 \\
\hline & $\mathrm{Tc}$ & 0.5 & 0.5 & 0.7 \\
\hline & $\mathbf{Y}$ & $<0.1$ & 0.3 & 0.1 \\
\hline & $\mathrm{Cr}$ & 0.1 & $<0.1$ & 0.1 \\
\hline & Co & 0.3 & 0.3 & 0.3 \\
\hline & $\mathrm{Fe}$ & 0.3 & 0.6 & 0.7 \\
\hline & $\mathrm{Mn}$ & 0.3 & 0.4 & 0.2 \\
\hline & $\mathrm{Zn}$ & 0.4 & 0.6 & 0.5 \\
\hline & $\mathrm{Zr}$ & 0.7 & 1.3 & 1.7 \\
\hline & $\mathrm{U}$ & 0.4 & 0.1 & 0.2 \\
\hline & $\mathrm{Pu}$ & 0.2 & 0.2 & 0.2 \\
\hline & $\mathrm{Am}$ & $<0.1$ & 0.3 & 0.4 \\
\hline Acidified & $\mathrm{Ce}$ & 0.3 & 0.1 & 0.4 \\
\hline \multirow[t]{13}{*}{ Supernate } & Cs & 9.4 & 21 & 34 \\
\hline & $\mathrm{Sr}$ & 0.1 & $<0.1$ & $<0.1$ \\
\hline & $\mathrm{Tc}$ & 0.4 & 0.3 & 0.4 \\
\hline & $\mathrm{Y}$ & $<0.1$ & 0.2 & 0.3 \\
\hline & $\mathrm{Cr}$ & 0.1 & 0.1 & 0.3 \\
\hline & Co & 0.4 & 0.3 & 0.3 \\
\hline & $\mathrm{Fe}$ & 0.3 & 0.8 & 0.8 \\
\hline & Mn & $<0.1$ & $<0.1$ & $<0.1$ \\
\hline & $\mathrm{Zn}$ & 0.4 & 0.6 & 0.6 \\
\hline & $\mathrm{Zr}$ & 0.3 & 0.2 & 0.3 \\
\hline & $\bar{U}$ & 0.2 & 0.7 & 0.8 \\
\hline & $\mathrm{Pu}$ & 0.8 & 1.0 & 0.9 \\
\hline & $\mathrm{Am}$ & 0.1 & 0.6 & 0.6 \\
\hline Alkaline & $\mathrm{Ce}$ & 5.6 & 9.8 & 9.4 \\
\hline \multirow[t]{12}{*}{ Supernate } & Cs & 0.4 & 0.8 & 1.6 \\
\hline & $\mathrm{Sr}_{\mathrm{r}}$ & 6.6 & 11 & 12 \\
\hline & Tc & 0.2 & $<0.1$ & $<0.1$ \\
\hline & $\mathrm{Y}$ & 5.2 & 9.3 & 11 \\
\hline & $\mathrm{Cr}$ & 0.4 & 0.6 & 0.4 \\
\hline & Co & $<0.1$ & $<0.1$ & $<0.1$ \\
\hline & $\mathrm{Fe}$ & 0.5 & 0.5 & 0.4 \\
\hline & $\mathrm{Mn}$ & $<0.1$ & $<0.1$ & $<0.1$ \\
\hline & $\mathrm{Zn}$ & 0.6 & 0.3 & 0.3 \\
\hline & $\mathrm{Zr}$ & 0.6 & 1.1 & 1.3 \\
\hline & $\mathrm{U}$ & 0.7 & 0.8 & 1.0 \\
\hline & Am & $<0.1$ & $<0.1$ & $<0.1$ \\
\hline
\end{tabular}


l. Durasil ${ }^{\mathrm{TM}} 230$ Resin. Durasil ${ }^{\mathrm{TM}} 230$ resin, obtained from Duratek Corporation, Beltsville, MD, was used as received. The air-dried weight, per water-wet milliliter, was $0.749 \mathrm{~g}$.
This resin sorbs zirconium and cesium from aciddissolved sludge solution, and cesium selectively from acidified supernate solution. Cesium, zirconium, iron, strontium, zinc, americium, manganese, and yttrium all sorb with at least double-digit $\mathrm{Kd}$ values from alkaline supernate solution.

Table 30. Durasil ${ }^{\mathrm{TM}} 230$ Resin: Distribution of 14 Elements from Three Simulant Solutions for Hanford Tank 102-SY

\begin{tabular}{|c|c|c|c|c|}
\hline \multirow[b]{2}{*}{ Solution } & \multirow[b]{2}{*}{ Element } & \multicolumn{3}{|c|}{ Kd Value for Specified Time } \\
\hline & & $30 \mathrm{~min}$ & $2 \mathrm{~h}$ & $6 \mathrm{~h}$ \\
\hline \multirow{14}{*}{$\begin{array}{l}\text { Acid-Dissolved } \\
\text { Sludge }\end{array}$} & $\mathrm{Ce}$ & 0.3 & 0.5 & 0.5 \\
\hline & Cs & 11 & 14 & 13 \\
\hline & $\mathrm{Sr}$ & 0.5 & 0.1 & 0.2 \\
\hline & Tc & 0.5 & 0.7 & 0.6 \\
\hline & $\mathrm{Y}$ & 0.2 & 0.2 & 0.2 \\
\hline & $\mathrm{Cr}$ & $<0.1$ & $<0.1$ & $<0.1$ \\
\hline & Co & 0.4 & 0.3 & 0.4 \\
\hline & $\mathrm{Fe}$ & $<0.1$ & 0.1 & 0.2 \\
\hline & $\mathrm{Mn}$ & $<0.1$ & $<0.1$ & $<0.1$ \\
\hline & $\mathrm{Zn}$ & 0.1 & 0.1 & 0.1 \\
\hline & $\mathrm{Zr}$ & 75 & 220 & 205 \\
\hline & $\mathrm{U}$ & 0.4 & 0.3 & 0.3 \\
\hline & $\mathrm{Pu}$ & 1.2 & 2.8 & 3.4 \\
\hline & Am & 0.3 & 0.1 & 0.2 \\
\hline Acidified & $\mathrm{Ce}$ & $<0.1$ & 0.1 & $<0.1$ \\
\hline \multirow{13}{*}{ Supernate } & Cs & 78 & 135 & 148 \\
\hline & $\mathrm{Sr}$ & 0.3 & 0.3 & 0.2 \\
\hline & $\mathrm{Tc}$ & 0.4 & 0.5 & 0.6 \\
\hline & $\mathbf{Y}$ & 0.3 & 0.3 & 0.3 \\
\hline & $\mathrm{Cr}$ & 0.4 & 0.6 & 0.7 \\
\hline & Co & 0.1 & $<0.1$ & 0.3 \\
\hline & $\mathrm{Fe}$ & $<0.1$ & 0.4 & 0.9 \\
\hline & $\mathrm{Mn}$ & 0.3 & 0.3 & 0.3 \\
\hline & $\mathrm{Zn}$ & 0.4 & 0.4 & 0.7 \\
\hline & $\mathrm{Zr}$ & 0.4 & 0.7 & 0.9 \\
\hline & $\mathrm{U}$ & 0.7 & 0.5 & 0.9 \\
\hline & $\mathrm{Pu}$ & $<0.1$ & 0.3 & 0.7 \\
\hline & $\mathrm{Am}$ & 0.1 & $<0.1$ & 0.1 \\
\hline Alkaline & $\mathrm{Ce}$ & 5.4 & 5.8 & 6.4 \\
\hline \multirow[t]{12}{*}{ Supernate } & Cs & 66 & 106 & 118 \\
\hline & $\mathrm{Sr}$ & 15 & 20 & 22 \\
\hline & Tc & $<0.1$ & $<0.1$ & $<0.1$ \\
\hline & $\mathrm{Y}$ & 7.3 & 9.8 & 11 \\
\hline & $\mathrm{Cr}$ & 0.5 & 0.6 & 0.7 \\
\hline & Co & 1.0 & 1.3 & 1.3 \\
\hline & $\mathrm{Fe}$ & 11 & 25 & 48 \\
\hline & $\mathrm{Mn}$ & 4.2 & 12 & 19 \\
\hline & $\mathrm{Zn}$ & 12 & 23 & 35 \\
\hline & $\mathrm{Zr}$ & 35 & 86 & 148 \\
\hline & $\vec{U}$ & 2.2 & 2.8 & 3.3 \\
\hline & Am & 0.9 & 7.8 & 14 \\
\hline
\end{tabular}


m. Bone Char. Bone char, produced by calcining cattle bones in the absence of air, was obtained from Stauffer Chemical Company, Westport, CT. Bone char is predominantly calcium phosphate. This absorber was used as received.

Bone char sorbs none of the 14 elements at useful levels from acid-dissolved sludge solution. It sorbs lanthanides, americium, technetium, cobalt, and zinc from acidified supernate solution, and it sorbs many elements well from alkaline supernate solution. Strontium, cerium, manganese, zirconium, yttrium, iron, and americium are especially well sorbed from alkaline supernate solution.

\begin{tabular}{|c|c|c|c|c|}
\hline \multirow[b]{2}{*}{ Solution } & \multirow[b]{2}{*}{ Element } & \multicolumn{3}{|c|}{ Kd Value for Specified Time } \\
\hline & & $30 \mathrm{~min}$ & $2 \mathrm{~h}$ & $6 \mathrm{~h}$ \\
\hline $\begin{array}{l}\text { Acid-Dissolved } \\
\text { Sludge }\end{array}$ & $\begin{array}{l}\mathrm{Ce} \\
\mathrm{Cs} \\
\mathrm{Sr} \\
\mathrm{Tc} \\
\mathrm{Y} \\
\mathrm{Cr} \\
\mathrm{Co} \\
\mathrm{Fe} \\
\mathrm{Mn} \\
\mathrm{Zn} \\
\mathrm{Zr} \\
\mathrm{U} \\
\mathrm{Pu} \\
\mathrm{Am}\end{array}$ & $\begin{array}{l}1.1 \\
1.1 \\
0.7 \\
4.0 \\
0.6 \\
0.7 \\
0.7 \\
0.4 \\
0.3 \\
0.4 \\
2.0 \\
1.5 \\
2.0 \\
0.6\end{array}$ & $\begin{array}{l}1.4 \\
1.0 \\
0.6 \\
4.0 \\
0.4 \\
0.5 \\
1.0 \\
0.2 \\
0.1 \\
0.2 \\
2.5 \\
0.9 \\
1.6 \\
0.4\end{array}$ & $\begin{array}{l}1.2 \\
0.9 \\
0.8 \\
3.9 \\
0.5 \\
0.7 \\
1.0 \\
0.3 \\
0.3 \\
0.3 \\
2.6 \\
1.1 \\
1.6 \\
0.3 \\
\end{array}$ \\
\hline $\begin{array}{l}\text { Acidified } \\
\text { Supernate }\end{array}$ & $\begin{array}{l}\mathrm{Ce} \\
\mathrm{Cs} \\
\mathrm{Sr} \\
\mathrm{Tc} \\
\mathrm{Y} \\
\mathrm{Cr} \\
\mathrm{Co} \\
\mathrm{Fe} \\
\mathrm{Mn} \\
\mathrm{Zn} \\
\mathrm{Zr} \\
\mathrm{U} \\
\mathrm{Pu} \\
\mathrm{Am}\end{array}$ & $\begin{array}{r}19 \\
0.2 \\
1.6 \\
12 \\
11 \\
0.2 \\
4.4 \\
0.1 \\
1.8 \\
8.3 \\
<0.1 \\
6.1 \\
0.1 \\
16\end{array}$ & $\begin{array}{r}33 \\
0.1 \\
2.0 \\
14 \\
18 \\
0.4 \\
5.5 \\
0.5 \\
2.5 \\
14 \\
0.2 \\
9.6 \\
0.2 \\
35\end{array}$ & $\begin{array}{r}53 \\
0.2 \\
1.9 \\
15 \\
26 \\
0.7 \\
6.9 \\
0.7 \\
2.9 \\
18 \\
0.3 \\
13 \\
1.1 \\
62 \\
\end{array}$ \\
\hline $\begin{array}{l}\text { Alkaline } \\
\text { Supernate }^{a}\end{array}$ & $\begin{array}{l}\mathrm{Ce} \\
\mathrm{Cs} \\
\mathrm{Sr} \\
\mathrm{Tc} \\
\mathrm{Y} \\
\mathrm{Cr} \\
\mathrm{Co} \\
\mathrm{Fe} \\
\mathrm{Mn} \\
\mathrm{Zn} \\
\mathrm{Zr} \\
\mathrm{U} \\
\mathrm{Am}\end{array}$ & $\begin{array}{r}914 \\
1.1 \\
>5 \mathrm{~K} \\
2.9 \\
172 \\
1.2 \\
14 \\
68 \\
62 \\
86 \\
204 \\
18 \\
57\end{array}$ & $\begin{array}{r}>1400 \\
3.1 \\
>11 \mathrm{~K} \\
5.1 \\
253 \\
2.9 \\
17 \\
202 \\
927 \\
153 \\
339 \\
24 \\
730\end{array}$ & $\begin{array}{r}>1500 \\
2.9 \\
>11 \mathrm{~K} \\
4.9 \\
339 \\
2.9 \\
18 \\
310 \\
3963 \\
215 \\
506 \\
28 \\
3084\end{array}$ \\
\hline
\end{tabular}

\footnotetext{
a After the absorber/solution contacts, all alkaline supernate solutions were passed through a $0.45-\mu \mathrm{m}$ filter to remove fine particles.
} 
n. Reillex ${ }^{\mathrm{TM}} H P Q$ Anion Exchange Resin. Reillex ${ }^{\mathrm{TM}}$ $\mathrm{HPQ}$, a strong-base polyvinylpyridine anion exchange resin, was obtained from Reilly Industries, Inc., Indianapolis, IN. This resin was converted from the as-received chloride form to the nitrate form and air-dried before use. The air-dried weight, per water-wet milliliter, was $0.352 \mathrm{~g}$.
This resin sorbs technetium well from all three solutions. It sorbs plutonium well and uranium and cerium moderately from acid-dissolved sludge solution. It sorbs americium surprisingly well from alkaline supernate solution. This higher than expected sorption of americium suggests that trivalent actinides might separate from lanthanides in such a solution.

Table 32. Reillex ${ }^{\mathrm{TM}}$ HPQ Anion Exchange Resin: Distribution of 14 Elements from Three Simulant Solutions for Hanford Tank 102-SY

\begin{tabular}{|c|c|c|c|c|}
\hline \multirow[b]{2}{*}{ Solution } & \multirow[b]{2}{*}{ Element } & \multicolumn{3}{|c|}{ Kd Value for Specified Tim } \\
\hline & & $30 \mathrm{~min}$ & $2 \mathbf{h}$ & $6 h$ \\
\hline Acid-Dissolved & $\mathrm{Ce}$ & 14 & 13 & 13 \\
\hline \multirow{13}{*}{ Sludge, $3 \mathrm{~g} \mathrm{Pu} / \mathrm{L}$} & Cs & 0.8 & 0.3 & 0.6 \\
\hline & $\mathrm{Sr}$ & 1.1 & 0.6 & 0.7 \\
\hline & Tc & 76 & 90 & 93 \\
\hline & $Y$ & 1.2 & 0.5 & 0.8 \\
\hline & $\mathrm{Cr}$ & 0.8 & 0.2 & 0.6 \\
\hline & Co & 0.9 & 0.2 & 0.2 \\
\hline & $\mathrm{Fe}$ & 0.5 & 0.5 & 0.5 \\
\hline & $\mathrm{Mn}$ & $<0.1$ & $<0.1$ & $<0.1$ \\
\hline & $\mathrm{Zn}$ & 0.3 & 0.2 & 0.2 \\
\hline & $\mathrm{Zr}$ & 4.3 & 4.2 & 4.2 \\
\hline & $\mathrm{U}$ & 17 & 19 & 18 \\
\hline & $\mathbf{P u}$ & 78 & 274 & 485 \\
\hline & Am & 3.9 & 2.4 & 2.4 \\
\hline Acid-Dissolved & $\mathrm{U}$ & 20 & 23 & 23 \\
\hline \multirow{2}{*}{ Sludge, $60 \mathrm{mg} \mathrm{Pu} / \mathrm{L}$} & $\mathrm{Pu}$ & 131 & 184 & 205 \\
\hline & Am & 4.6 & 3.6 & 3.8 \\
\hline Acidified & $\mathrm{Ce}$ & 0.4 & 0.6 & 0.5 \\
\hline \multirow{13}{*}{ Supernate } & Cs & 1.0 & 0.8 & 0.8 \\
\hline & $\mathrm{Sr}$ & 0.1 & 0.3 & 0.1 \\
\hline & $\mathrm{Tc}$ & 250 & 280 & 274 \\
\hline & $\mathrm{Y}$ & 0.8 & 1.0 & 0.6 \\
\hline & $\mathrm{Cr}$ & $<0.1$ & $<0.1$ & $<0.1$ \\
\hline & Co & 0.9 & 1.0 & 0.8 \\
\hline & $\mathrm{Fe}$ & 0.7 & 0.8 & 1.2 \\
\hline & $\mathrm{Mn}$ & $<0.1$ & $<0.1$ & $<0.1$ \\
\hline & $\mathrm{Zn}$ & 0.6 & 0.5 & 0.6 \\
\hline & $\mathrm{Zr}$ & 0.2 & 0.4 & 0.4 \\
\hline & $\mathrm{U}$ & 8.4 & 8.9 & 8.9 \\
\hline & $\mathrm{Pu}$ & 0.7 & 0.9 & 0.7 \\
\hline & $\mathrm{Am}$ & 1.0 & 0.9 & 0.8 \\
\hline Alkaline & $\mathrm{Ce}$ & $<0.1$ & 0.7 & 1.0 \\
\hline \multirow{12}{*}{ Supernate } & Cs & $<0.1$ & $<0.1$ & $<0.1$ \\
\hline & $\mathrm{Sr}$ & 0.4 & 0.6 & 0.6 \\
\hline & Tc & 314 & 372 & 370 \\
\hline & $\mathrm{Y}$ & 2.3 & 3.4 & 4.1 \\
\hline & $\mathrm{Cr}$ & 0.1 & 0.2 & 0.2 \\
\hline & Co & 2.2 & 2.7 & 2.7 \\
\hline & $\mathrm{Fe}$ & 3.4 & 3.7 & 4.4 \\
\hline & $\mathrm{Mn}$ & 14 & 18 & 26 \\
\hline & $\mathrm{Zn}$ & $<0.1$ & $<0.1$ & $<0.1$ \\
\hline & $\mathrm{Zr}$ & 1.3 & 1.5 & 1.8 \\
\hline & $\mathrm{U}$ & 0.2 & 0.4 & 0.7 \\
\hline & Am & 4.5 & 7.5 & 21 \\
\hline
\end{tabular}


o. Ionac ${ }^{\mathrm{TM}}$ SR-3 Anion Exchange Resin. Ionac ${ }^{\mathrm{TM}}$ SR-3, a macroporous strong-base anion exchange resin with trimethyl amine as the functional group, was obtained from Sybron Chemicals, Inc., Birmingham, NJ. The air-dried weight, per water-wet milliliter, was $0.359 \mathrm{~g}$.
This resin sorbs plutonium, technetium, uranium, and cerium from acid-dissolved sludge solution. Technetium sorbs well from the other two solutions.

\begin{tabular}{|c|c|c|c|c|}
\hline \multicolumn{3}{|c|}{$\begin{array}{l}\text { Table 33. Ionac }{ }^{\mathrm{TM}} \text { SR-3 Anion Exchange Resin: Distribution of } 14 \\
\text { Elements from Three Simulant Solutions for Hanford Tank 102-SY }\end{array}$} & & \\
\hline \multirow[b]{2}{*}{ Solution } & \multirow[b]{2}{*}{ Element } & \multicolumn{3}{|c|}{ Kd Value for Specified Time } \\
\hline & & $30 \mathrm{~min}$ & $2 \mathrm{~h}$ & $6 \mathrm{~h}$ \\
\hline \multirow{14}{*}{$\begin{array}{l}\text { Acid-Dissolved } \\
\text { Sludge }\end{array}$} & $\mathrm{Ce}$ & 6.6 & 13 & 21 \\
\hline & Cs & $<0.1$ & $<0.1$ & $<0.1$ \\
\hline & $\mathrm{Sr}$ & 0.2 & 0.6 & 0.3 \\
\hline & Tc & 32 & 44 & 43 \\
\hline & $\mathrm{Y}$ & 1.5 & 1.7 & 1.4 \\
\hline & $\mathrm{Cr}$ & 0.5 & 0.9 & 0.7 \\
\hline & Co & $<0.1$ & 0.3 & 0.3 \\
\hline & $\mathrm{Fe}$ & 0.3 & 0.5 & 0.6 \\
\hline & $\mathbf{M n}$ & $<0.1$ & $<0.1$ & $<0.1$ \\
\hline & $\mathrm{Zn}$ & 0.1 & 0.2 & 0.5 \\
\hline & $\mathrm{Zr}$ & 1.4 & 2.8 & 2.4 \\
\hline & $\mathrm{U}$ & 12 & 21 & 37 \\
\hline & $\mathrm{Pu}$ & 21 & 64 & 207 \\
\hline & Am & 2.6 & 3.7 & 4.3 \\
\hline \multirow{14}{*}{$\begin{array}{l}\text { Acidified } \\
\text { Supernate }\end{array}$} & $\mathrm{Ce}$ & 3.5 & 3.5 & 3.4 \\
\hline & $\mathrm{Cs}$ & 0.5 & 0.5 & 0.4 \\
\hline & $\mathrm{Sr}$ & 1.0 & 0.7 & 0.9 \\
\hline & $\mathrm{Tc}$ & 121 & 123 & 125 \\
\hline & $\mathrm{Y}$ & 0.7 & 0.6 & 0.7 \\
\hline & $\mathrm{Cr}$ & $<0.1$ & $<0.1$ & $<0.1$ \\
\hline & Co & 0.8 & 0.8 & 0.7 \\
\hline & $\mathrm{Fe}$ & $<0.1$ & 0.3 & 0.1 \\
\hline & $\mathrm{Mn}$ & 0.3 & 0.5 & 0.3 \\
\hline & $\mathrm{Zn}$ & 0.3 & 0.4 & 0.4 \\
\hline & $\mathrm{Zr}$ & 0.5 & 0.2 & 0.3 \\
\hline & $\mathrm{U}$ & 1.2 & 1.6 & 1.7 \\
\hline & $\mathrm{Pu}$ & 0.5 & 0.1 & 0.2 \\
\hline & $\mathrm{Am}$ & 0.5 & 0.9 & 0.8 \\
\hline \multirow{13}{*}{$\begin{array}{l}\text { Alkaline } \\
\text { Supernate }\end{array}$} & $\mathrm{Ce}$ & $<0.1$ & $<0.1$ & 0.4 \\
\hline & $\mathrm{Cs}$ & 0.8 & 0.4 & 0.3 \\
\hline & $\mathrm{Sr}$ & 0.7 & 1.3 & 1.4 \\
\hline & Tc & 222 & 272 & 258 \\
\hline & $\mathrm{Y}$ & 0.6 & 1.2 & 1.3 \\
\hline & $\mathrm{Cr}$ & 1.1 & 0.6 & 0.8 \\
\hline & Co & 0.5 & 0.3 & 0.2 \\
\hline & $\mathrm{Fe}$ & 0.4 & 0.4 & 0.8 \\
\hline & $\mathrm{Mn}$ & 0.1 & 0.1 & 0.6 \\
\hline & $\mathrm{Zn}$ & $<0.1$ & $<0.1$ & $<0.1$ \\
\hline & $\mathrm{Zr}$ & 0.9 & 0.4 & 0.6 \\
\hline & $\mathrm{U}$ & 0.5 & 0.4 & 0.9 \\
\hline & Am & $<0.1$ & $<0.1$ & $<0.1$ \\
\hline
\end{tabular}


p. Purolite ${ }^{\mathrm{TM}}$ A-520-E Anion Exchange Resin. Purolite ${ }^{\mathrm{TM}}$ A-520-E, a macroporous strong-base anion exchange resin with triethyl amine as the functional group, was obtained from the Purolite Company, Bala Cynwyd, PA. The air-dried weight, per water-wet milliliter, was $0.351 \mathrm{~g}$.
This resin sorbs plutonium, technetium, uranium, and cerium from acid-dissolved sludge solution. It sorbs technetium well from the other two solutions.

\begin{tabular}{|c|c|c|c|c|}
\hline \multirow[b]{2}{*}{ Solution } & \multirow[b]{2}{*}{ Element } & \multicolumn{3}{|c|}{ Kd Value for Specified Time } \\
\hline & & $30 \mathrm{~min}$ & $2 \mathrm{~h}$ & $6 \mathrm{~h}$ \\
\hline $\begin{array}{l}\text { Acid-Dissolved } \\
\text { Sludge, } 3 \text { g Pu/L }\end{array}$ & $\begin{array}{l}\mathrm{Ce} \\
\mathrm{Cs} \\
\mathrm{Sr} \\
\mathrm{Tc} \\
\mathrm{Y} \\
\mathrm{Cr} \\
\mathrm{Co} \\
\mathrm{Fe} \\
\mathrm{Mn} \\
\mathrm{Zn} \\
\mathrm{Zr} \\
\mathrm{U} \\
\mathrm{Pu} \\
\mathrm{Am}\end{array}$ & $\begin{array}{r}7.8 \\
<0.1 \\
<0.1 \\
32 \\
1.4 \\
0.5 \\
0.2 \\
0.2 \\
<0.1 \\
0.3 \\
2.1 \\
10.1 \\
13 \\
2.9\end{array}$ & $\begin{array}{r}14 \\
<0.1 \\
0.1 \\
57 \\
1.1 \\
0.4 \\
0.2 \\
0.4 \\
<0.1 \\
0.3 \\
2.4 \\
24 \\
51 \\
4.2\end{array}$ & $\begin{array}{r}21 \\
0.1 \\
<0.1 \\
77 \\
1.1 \\
0.5 \\
0.3 \\
0.4 \\
<0.1 \\
0.3 \\
2.3 \\
35 \\
415 \\
3.2\end{array}$ \\
\hline $\begin{array}{l}\text { Acid-Dissolved } \\
\text { Sludge, } 60 \mathrm{mg} \mathrm{Pu} / \mathrm{L}\end{array}$ & $\begin{array}{l}\mathrm{U} \\
\mathrm{Pu} \\
\mathrm{Am}\end{array}$ & $\begin{array}{r}9.9 \\
45 \\
2.7\end{array}$ & $\begin{array}{r}22 \\
186 \\
4.2\end{array}$ & $\begin{array}{r}39 \\
260 \\
3.7\end{array}$ \\
\hline $\begin{array}{l}\text { Acidified } \\
\text { Supernate }\end{array}$ & $\begin{array}{l}\mathrm{Ce} \\
\mathrm{Cs} \\
\mathrm{Sr} \\
\mathrm{Tc} \\
\mathrm{Y} \\
\mathrm{Cr} \\
\mathrm{Co} \\
\mathrm{Fe} \\
\mathrm{Mn} \\
\mathrm{Zn} \\
\mathrm{Zr} \\
\mathrm{U} \\
\mathrm{Pu} \\
\mathrm{Am}\end{array}$ & $\begin{array}{r}4.8 \\
0.7 \\
0.6 \\
205 \\
0.6 \\
<0.1 \\
0.9 \\
0.5 \\
<0.1 \\
0.5 \\
0.7 \\
3.3 \\
0.3 \\
0.6\end{array}$ & $\begin{array}{r}5.3 \\
0.2 \\
0.4 \\
320 \\
0.4 \\
<0.1 \\
0.5 \\
0.6 \\
0.2 \\
0.4 \\
0.3 \\
4.6 \\
0.1 \\
0.7\end{array}$ & $\begin{array}{r}6.1 \\
0.3 \\
0.5 \\
380 \\
0.5 \\
<0.1 \\
0.6 \\
0.5 \\
0.2 \\
0.2 \\
0.3 \\
4.3 \\
0.2 \\
0.6\end{array}$ \\
\hline $\begin{array}{l}\text { Alkaline } \\
\text { Supernate }\end{array}$ & $\begin{array}{l}\mathrm{Ce} \\
\mathrm{Cs} \\
\mathrm{Sr} \\
\mathrm{Tc} \\
\mathrm{Y} \\
\mathrm{Cr} \\
\mathrm{Co} \\
\mathrm{Fe} \\
\mathrm{Mn} \\
\mathrm{Zn} \\
\mathrm{Zr} \\
\mathrm{U} \\
\mathrm{Am}\end{array}$ & $\begin{array}{r}<0.1 \\
0.8 \\
0.5 \\
371 \\
0.5 \\
0.8 \\
1.0 \\
0.4 \\
<0.1 \\
0.3 \\
0.8 \\
0.8 \\
<0.1\end{array}$ & $\begin{array}{r}0.2 \\
0.2 \\
1.2 \\
585 \\
1.2 \\
0.3 \\
0.3 \\
0.4 \\
0.2 \\
<0.1 \\
0.3 \\
0.7 \\
<0.1 \\
\end{array}$ & $\begin{array}{r}<0.1 \\
0.3 \\
1.5 \\
620 \\
2.0 \\
0.6 \\
0.4 \\
1.0 \\
1.1 \\
<0.1 \\
0.6 \\
0.8 \\
<0.1 \\
\end{array}$ \\
\hline
\end{tabular}


q. Ionac ${ }^{\mathrm{TM}}$ SR-6 Anion Exchange Resin. Ionac ${ }^{\mathrm{TM}}$ SR-6, a macroporous strong-base anion exchange resin with tributyl amine as the functional group, was obtained from Sybron Chemicals, Inc., Birmingham, NJ. The air-dried weight, per water-wet milliliter, was $0.462 \mathrm{~g}$. Ionac ${ }^{\mathrm{TM}}$ SR-6 resin has been reported ${ }^{11}$ to offer greatly increased selectivity for sorbing nitrate ion from groundwater.
This resin quite selectively sorbs technetium from all three solutions; however, the much-lower $\mathrm{Kd}$ value for sorption from acid-dissolved sludge solution suggests that technetium may elute more easily with nitric acid from this resin than from other anion exchange resins.

\begin{tabular}{|c|c|c|c|c|}
\hline \multirow[b]{2}{*}{ Solution } & \multirow[b]{2}{*}{ Element } & \multicolumn{3}{|c|}{ Kd Value for Specified Time } \\
\hline & & $30 \mathrm{~min}$ & 2 h & $6 \mathrm{~h}$ \\
\hline $\begin{array}{l}\text { Acid-Dissolved } \\
\text { Sludge }\end{array}$ & $\begin{array}{l}\mathrm{Ce} \\
\mathrm{Cs} \\
\mathrm{Sr} \\
\mathrm{Tc} \\
\mathrm{Y} \\
\mathrm{Cr} \\
\mathrm{Co} \\
\mathrm{Fe} \\
\mathrm{Mn} \\
\mathrm{Zn} \\
\mathrm{Zr} \\
\mathrm{U} \\
\mathrm{Pu} \\
\mathrm{Am}\end{array}$ & $\begin{array}{r}0.6 \\
0.3 \\
0.1 \\
2.2 \\
0.1 \\
0.2 \\
<0.1 \\
0.1 \\
<0.1 \\
0.2 \\
<0.1 \\
0.2 \\
0.3 \\
0.1 \\
\end{array}$ & $\begin{array}{r}0.7 \\
0.3 \\
0.7 \\
4.6 \\
0.4 \\
0.3 \\
<0.1 \\
0.3 \\
<0.1 \\
0.2 \\
0.5 \\
0.8 \\
0.5 \\
0.3 \\
\end{array}$ & $\begin{array}{r}0.7 \\
0.4 \\
0.4 \\
8.3 \\
0.3 \\
0.5 \\
<0.1 \\
0.8 \\
<0.1 \\
0.3 \\
0.6 \\
0.7 \\
0.5 \\
0.3 \\
\end{array}$ \\
\hline $\begin{array}{l}\text { Acidified } \\
\text { Supernate }\end{array}$ & $\begin{array}{l}\mathrm{Ce} \\
\mathrm{Cs} \\
\mathrm{Sr} \\
\mathrm{Tc} \\
\mathrm{Y} \\
\mathrm{Cr} \\
\mathrm{Co} \\
\mathrm{Fe} \\
\mathrm{Mn} \\
\mathrm{Zn} \\
\mathrm{Zr} \\
\mathrm{U} \\
\mathrm{Pu} \\
\mathrm{Am} \\
\end{array}$ & $\begin{array}{r}<0.1 \\
0.3 \\
0.1 \\
151 \\
0.4 \\
0.4 \\
0.2 \\
0.1 \\
0.5 \\
0.2 \\
0.2 \\
0.6 \\
0.7 \\
<0.1 \\
\end{array}$ & $\begin{array}{r}0.2 \\
0.1 \\
0.3 \\
394 \\
0.5 \\
0.6 \\
0.4 \\
<0.1 \\
0.2 \\
0.3 \\
0.3 \\
1.3 \\
0.2 \\
<0.1 \\
\end{array}$ & $\begin{array}{r}0.1 \\
0.1 \\
0.3 \\
619 \\
0.4 \\
0.8 \\
0.6 \\
0.2 \\
0.2 \\
0.5 \\
0.3 \\
1.7 \\
0.5 \\
<0.1 \\
\end{array}$ \\
\hline $\begin{array}{l}\text { Alkaline } \\
\text { Supernate }\end{array}$ & $\begin{array}{l}\mathrm{Ce} \\
\mathrm{Cs} \\
\mathrm{Sr} \\
\mathrm{Tc} \\
\mathrm{Y} \\
\mathrm{Cr} \\
\mathrm{Co} \\
\mathrm{Fe} \\
\mathrm{Mn} \\
\mathrm{Zn} \\
\mathrm{Zr} \\
\mathrm{U} \\
\mathrm{Am}\end{array}$ & $\begin{array}{r}<0.1 \\
0.3 \\
0.6 \\
191 \\
0.8 \\
0.4 \\
0.1 \\
0.4 \\
<0.1 \\
0.3 \\
0.5 \\
0.3 \\
<0.1\end{array}$ & $\begin{array}{r}1.0 \\
<0.1 \\
3.2 \\
385 \\
3.4 \\
0.3 \\
0.1 \\
0.6 \\
<0.1 \\
0.3 \\
0.5 \\
0.6 \\
<0.1\end{array}$ & $\begin{array}{r}2.0 \\
<0.1 \\
4.4 \\
520 \\
5.4 \\
0.2 \\
0.1 \\
0.7 \\
<0.1 \\
0.3 \\
0.7 \\
0.8 \\
<0.1\end{array}$ \\
\hline
\end{tabular}


r. SRS Resorcinol/Formaldehyde Resin. Dr. Jane Bibler of Westinghouse Savannah River Company provided this sample of batch BSC-187 resin and recommended that we convert it from the as-received potassium form to a sodium form. We did this by passing ten volumes of $10 \%$ $\mathrm{NaOH}$ solution through a column of potassium-form resin, rinsing the column with 15 column volumes of water, and finally air-drying the resin. The air-dried weight, per waterwet milliliter, was $0.254 \mathrm{~g}$.
This resin sorbs technetium and cesium somewhat from acid-dissolved sludge solution. It sorbs cesium well from acidified supernate solution, but cesium is accompanied by significant levels of many other elements. Sorption of cesium from alkaline supernate solution is even higher; however, many other elements cosorb with moderate to high $\mathrm{Kd}$ values.

\begin{tabular}{|c|c|c|c|c|}
\hline \multirow[b]{2}{*}{ Solution } & \multirow[b]{2}{*}{ Element } & \multicolumn{3}{|c|}{ Kd Value for Specified Time } \\
\hline & & $30 \mathrm{~min}$ & $2 \mathrm{~h}$ & $6 \mathrm{~h}$ \\
\hline \multirow{14}{*}{$\begin{array}{l}\text { Acid-Dissolved } \\
\text { Sludge }\end{array}$} & $\mathrm{Ce}$ & 0.8 & 1.0 & 1.1 \\
\hline & Cs & 2.7 & 4.5 & 5.9 \\
\hline & $\mathrm{Sr}$ & 1.2 & 0.8 & 1.1 \\
\hline & Tc & 2.7 & 4.5 & 11 \\
\hline & $\mathrm{Y}$ & 0.7 & 1.0 & 1.1 \\
\hline & $\mathrm{Cr}$ & 0.8 & 0.9 & 1.0 \\
\hline & Co & 1.0 & 1.2 & 1.3 \\
\hline & $\mathrm{Fe}$ & 0.2 & 0.5 & 0.3 \\
\hline & Mn & 0.3 & 0.4 & 0.5 \\
\hline & $\mathrm{Zn}$ & 0.2 & 0.5 & 0.5 \\
\hline & $\mathrm{Zr}$ & 1.3 & 0.9 & 1.5 \\
\hline & $\mathbf{U}$ & 0.2 & $<0.1$ & 0.3 \\
\hline & $\mathbf{P u}$ & $<0.1$ & 0.1 & 0.1 \\
\hline & Am & $<0.1$ & 0.4 & 0.1 \\
\hline \multirow{14}{*}{$\begin{array}{l}\text { Acidified } \\
\text { Supernate }\end{array}$} & $\mathrm{Ce}$ & 14 & 26 & 42 \\
\hline & Cs & 75 & 85 & 66 \\
\hline & $\mathrm{Sr}$ & 4.8 & 2.6 & 1.5 \\
\hline & Tc & 1.0 & 1.5 & 1.8 \\
\hline & $\mathrm{Y}$ & 12 & 21 & 36 \\
\hline & $\mathrm{Cr}$ & 0.3 & 1.0 & 0.8 \\
\hline & Co & 12 & 15 & 7.7 \\
\hline & $\mathrm{Fe}$ & 0.2 & 0.5 & 0.7 \\
\hline & $\mathrm{Mn}$ & 5.9 & 4.7 & 2.2 \\
\hline & $\mathrm{Zn}$ & 9.3 & 11 & 9.0 \\
\hline & $\mathrm{Zr}$ & $<0.1$ & $<0.1$ & 0.1 \\
\hline & $\mathbf{U}$ & 7.5 & 13 & 22 \\
\hline & $\mathrm{Pu}$ & 0.2 & 0.9 & 1.2 \\
\hline & $\mathrm{Am}$ & 11 & 19 & 27 \\
\hline \multirow{13}{*}{$\begin{array}{l}\text { Alkaline } \\
\text { Supernate }\end{array}$} & $\mathrm{Ce}$ & 1.1 & 1.1 & 1.4 \\
\hline & $\mathrm{Cs}$ & 314 & 599 & 847 \\
\hline & $\mathrm{Sr}$ & 20 & 31 & 76 \\
\hline & Tc & $<0.1$ & $<0.1$ & $<0.1$ \\
\hline & $\mathrm{Y}$ & 4.0 & 8.0 & 19 \\
\hline & $\mathrm{Cr}$ & 3.9 & 16 & 71 \\
\hline & Co & 2.9 & 7.5 & 13 \\
\hline & $\mathrm{Fe}$ & 33 & 40 & 46 \\
\hline & $\mathrm{Mn}$ & $<0.1$ & 0.5 & 1.0 \\
\hline & $\mathrm{Zn}$ & 6.8 & 16 & 31 \\
\hline & $\mathrm{Zr}$ & 41 & 81 & 123 \\
\hline & $\mathrm{U}$ & 7.2 & 15 & 41 \\
\hline & $\mathrm{Am}$ & $<0.1$ & $<0.1$ & $<0.1$ \\
\hline
\end{tabular}


2. Experimental Absorbers. Seven experimental absorbers were included in our study. Two of these are experimental resins produced by Sybron Chemicals, one is a cryptand polymer developed at Los Alamos, and one is a phosphinic acid resin developed at the University of Tennessee.

The remaining three JSK experimental anion exchange resins were custom-synthesized as part of a Los Alamos effort to develop new plutonium-selective resins. Their composition cannot be disclosed until the development program is complete and the Laboratory's patent position is secured. We can state, however, that although these resins involve a new concept for anion exchange, they can be produced by modifying existing commercial resins. For this reason, production on a commercial scale may be considerably less difficult than would be the case for an entirely new resin. Because these three resins were not stable in alkaline solution, they were studied only for sorption from acid-dissolved sludge and acidified supernate solutions. 
a. Sybron (Et) ${ }_{3} N$ Anion Exchange Resin. This experimental, macroporous strong-base anion exchange resin with triethyl amine as the functional group was obtained from Sybron Chemicals, Birmingham, NJ. The air-dried weight, per water-wet milliliter, was $0.365 \mathrm{~g}$. A previous study ${ }^{12}$ found that this resin offers a significant increase over $(\mathrm{Me})_{3} \mathrm{~N}$ resin for sorbing $\mathrm{Pu}(\mathrm{IV})$ from dilute nitric acid.
The behavior of this experimental resin in all three solutions is fairly similar to that of Purolite ${ }^{\mathrm{TM}}$ A-520-E resin (its commercial equivalent).

\begin{tabular}{|c|c|c|c|c|}
\hline & & Kd Val & r Spe & I Time \\
\hline Solution & Element & $30 \mathrm{~min}$ & $2 \mathrm{~h}$ & $6 h$ \\
\hline Acid-Dissolved & $\mathrm{Ce}$ & 7.4 & 9.6 & 7.5 \\
\hline Sludge & Cs & 0.2 & 0.1 & 0.2 \\
\hline & $\mathrm{Sr}$ & 0.1 & 0.2 & 0.3 \\
\hline & Tc & 31 & 51 & 53 \\
\hline & $\mathrm{Y}$ & 0.6 & 0.6 & 0.3 \\
\hline & $\mathrm{Cr}$ & 0.3 & 0.1 & 0.4 \\
\hline & Co & $<0.1$ & $<0.1$ & $<0.1$ \\
\hline & $\mathrm{Fe}$ & 0.1 & 0.1 & 0.4 \\
\hline & Mn & $<0.1$ & $<0.1$ & 0.3 \\
\hline & $\mathrm{Zn}$ & 0.3 & 0.2 & 0.5 \\
\hline & $\mathrm{Zr}$ & 2.4 & 2.6 & 2.0 \\
\hline & $\mathrm{U}$ & 13 & 22 & 22 \\
\hline & $\mathrm{Pu}$ & 24 & 114 & 839 \\
\hline & $\mathrm{Am}$ & 2.6 & 1.4 & 0.8 \\
\hline Acidified & $\mathrm{Ce}$ & 0.7 & 0.7 & 0.8 \\
\hline Supernate & Cs & $<0.1$ & $<0.1$ & $<0.1$ \\
\hline & $\mathrm{Sr}$ & 0.2 & 0.4 & 0.3 \\
\hline & $\mathrm{Tc}$ & 264 & 405 & 440 \\
\hline & $Y$ & 0.2 & 0.5 & 0.4 \\
\hline & $\mathrm{Cr}$ & 0.8 & 0.9 & 0.8 \\
\hline & Co & 0.2 & 0.5 & 0.4 \\
\hline & $\mathrm{Fe}$ & 0.1 & 0.2 & 0.2 \\
\hline & $\mathrm{Mn}$ & 0.3 & 0.3 & 0.4 \\
\hline & $\mathrm{Zn}$ & 0.5 & 0.5 & 0.3 \\
\hline & $\mathrm{Zr}$ & 0.1 & 0.1 & 0.3 \\
\hline & $\mathrm{U}$ & 2.8 & 3.1 & 3.1 \\
\hline & $\mathrm{Pu}$ & $<0.1$ & $<0.1$ & 0.2 \\
\hline & Am & 0.3 & 0.4 & 0.2 \\
\hline Alkaline & $\mathrm{Ce}$ & 0.3 & 0.8 & 2.0 \\
\hline Supernate & $\mathrm{Cs}$ & 0.1 & $<0.1$ & $<0.1$ \\
\hline & $\mathrm{Sr}$ & 5.3 & 5.6 & 7.3 \\
\hline & Tc & 279 & 446 & 521 \\
\hline & Y & 1.6 & 2.9 & 7.4 \\
\hline & $\mathrm{Cr}$ & $<0.1$ & $<0.1$ & $<0.1$ \\
\hline & Co & 0.2 & $<0.1$ & 0.3 \\
\hline & $\mathrm{Fe}$ & 4.4 & 5.2 & 6.7 \\
\hline & $\mathrm{Mn}$ & $<0.1$ & $<0.1$ & 0.6 \\
\hline & $\mathrm{Zn}$ & 0.4 & 0.1 & 0.1 \\
\hline & $\mathrm{Zr}$ & 2.0 & 3.3 & 4.6 \\
\hline & $\mathrm{U}$ & $<0.1$ & 0.3 & 0.3 \\
\hline & Am & $<0.1$ & $<0.1$ & $<0.1$ \\
\hline
\end{tabular}


b. Sybron (Pr) $)_{3} N$ Anion Exchange Resin. This experimental, macroporous strong-base anion exchange resin with tripropyl amine as the functional group was obtained from Sybron Chemicals, Birmingham, NJ. The air-dried weight, per water-wet milliliter, was $0.406 \mathrm{~g}$.
This resin sorbs technetium from all three solutions. The lower $\mathrm{Kd}$ value for sorption from acid-dissolved sludge solution suggests that nitric acid may elute technetium more easily from this resin than from most other anion exchange resins.

Table 38. Sybron $(\mathrm{Pr})_{3} \mathrm{~N}$ Anion Exchange Resin: Distribution of 14 Elements from Three Simulant Solutions for Hanford Tank 102-SY

\begin{tabular}{|c|c|c|c|c|}
\hline \multirow[b]{2}{*}{ Solution } & \multirow[b]{2}{*}{ Element } & \multicolumn{3}{|c|}{ Kd Value for Specified Time } \\
\hline & & $30 \mathrm{~min}$ & $2 \mathbf{h}$ & $6 \mathrm{~h}$ \\
\hline Acid-Dissolved & $\mathrm{Ce}$ & 1.2 & 1.7 & 2.2 \\
\hline \multirow{13}{*}{ Sludge } & Cs & 0.1 & 0.5 & 0.3 \\
\hline & $\mathrm{Sr}$ & $<0.1$ & 0.4 & 0.2 \\
\hline & Tc & 11 & 26 & 45 \\
\hline & $Y$ & 0.2 & 0.5 & 0.3 \\
\hline & $\mathrm{Cr}$ & $<0.1$ & 0.8 & 0.6 \\
\hline & Co & 0.3 & 0.1 & 0.1 \\
\hline & $\mathrm{Fe}$ & 0.1 & 0.3 & 0.3 \\
\hline & $\mathrm{Mn}$ & 0.1 & $<0.1$ & $<0.1$ \\
\hline & $\mathrm{Zn}$ & $<0.1$ & 0.4 & 0.2 \\
\hline & $\mathrm{Zr}$ & 0.5 & 1.0 & 1.8 \\
\hline & $U$ & 1.9 & 3.5 & 7.1 \\
\hline & $\mathrm{Pu}$ & 0.7 & 1.7 & 3.2 \\
\hline & $\mathrm{Am}$ & 0.3 & 0.7 & 0.9 \\
\hline Acidified & $\mathrm{Ce}$ & 0.4 & 0.1 & 0.2 \\
\hline \multirow[t]{13}{*}{ Supernate } & Cs & $<0.1$ & $<0.1$ & $<0.1$ \\
\hline & $\mathrm{Sr}$ & 0.3 & 0.2 & 0.3 \\
\hline & $\mathrm{Tc}$ & 401 & 777 & 992 \\
\hline & $\mathrm{Y}$ & 0.4 & 0.5 & 0.4 \\
\hline & $\mathrm{Cr}$ & 0.6 & 0.6 & 0.6 \\
\hline & Co & 0.4 & 0.6 & 0.4 \\
\hline & $\mathrm{Fe}$ & 0.3 & 0.2 & 0.1 \\
\hline & $\mathrm{Mn}$ & $<0.1$ & 0.2 & 0.1 \\
\hline & $\mathrm{Zn}$ & 0.7 & 0.4 & 0.4 \\
\hline & $\mathrm{Zr}$ & 1.2 & 0.4 & 0.7 \\
\hline & $\mathbf{U}$ & 3.5 & 4.2 & 5.4 \\
\hline & $\mathrm{Pu}$ & 0.5 & 0.4 & 0.3 \\
\hline & $\mathrm{Am}$ & 0.3 & 0.1 & 0.1 \\
\hline Alkaline & $\mathrm{Ce}$ & 1.5 & 1.3 & 1.2 \\
\hline \multirow{12}{*}{ Supernate } & Cs & 0.3 & $<0.1$ & $<0.1$ \\
\hline & $\mathrm{Sr}$ & 2.2 & 3.0 & 3.5 \\
\hline & $\mathrm{Tc}$ & 262 & 477 & 554 \\
\hline & $\mathrm{Y}$ & 1.9 & 2.8 & 4.0 \\
\hline & $\mathrm{Cr}$ & $<0.1$ & 0.1 & 0.2 \\
\hline & Co & $<0.1$ & $<0.1$ & $<0.1$ \\
\hline & $\mathrm{Fe}$ & 1.4 & 1.7 & 2.8 \\
\hline & $\mathrm{Mn}$ & $<0.1$ & 0.9 & 2.8 \\
\hline & $\mathrm{Zn}$ & 0.2 & $<0.1$ & $<0.1$ \\
\hline & $\mathrm{Zr}$ & 0.5 & 0.9 & 1.2 \\
\hline & $\mathrm{U}$ & 0.4 & 0.4 & 0.4 \\
\hline & Am & $<0.1$ & $<0.1$ & 1.1 \\
\hline
\end{tabular}


c. JSK-I Anion Exchange Resin. From acid-dissolved sludge solution, this resin sorbs plutonium strongly, whereas it sorbs cerium, technetium, and uranium at lower, but useful levels. It sorbs zirconium and yttrium poorly from both solutions. From acidified supernate solution, it sorbs only technetium well. This resin could not be studied with alkaline supernate solution because it is unstable in strongly basic solution.

\begin{tabular}{lcccc}
\hline \hline Table 39. JSK-1 Anion Exchange Resin: & Distribution of 14 & Elements \\
from Two Simulant Solutions for Hanford Tank 102-SY & \\
\hline & & \multicolumn{3}{c}{ Kd Value for Specified Time } \\
\cline { 2 - 5 } Solution & Element & 30 min & $\mathbf{2 ~ h}$ & $\mathbf{6 h}$ \\
\hline Acid-Dissolved & $\mathrm{Ce}$ & 28 & 29 & 27 \\
Sludge, 3 g Pu/L & $\mathrm{Cs}$ & 0.4 & 0.1 & 0.2 \\
& $\mathrm{Sr}$ & 1.2 & 1.3 & 1.1 \\
& $\mathrm{Tc}$ & 32 & 32 & 30 \\
& $\mathrm{Y}$ & 1.7 & 1.2 & 1.1 \\
& $\mathrm{Cr}$ & 0.5 & 0.4 & 0.4 \\
& $\mathrm{Co}$ & $<0.1$ & 0.3 & 0.1 \\
& $\mathrm{Fe}$ & 0.1 & 0.1 & 0.2 \\
& $\mathrm{Mn}$ & $<0.1$ & $<0.1$ & $<0.1$ \\
& $\mathrm{Zn}$ & 1.6 & 1.0 & 0.9 \\
& $\mathrm{Zr}$ & 4.7 & 4.4 & 2.8 \\
& $\mathrm{U}$ & 14 & 13 & 12 \\
& $\mathrm{Pu}$ & 83 & 143 & 196 \\
& $\mathrm{Am}$ & 7.4 & 6.5 & 5.9 \\
& $\mathrm{U}$ & 13 & 12 & 11 \\
Acid-Dissolved & $\mathrm{Pu}$ & 178 & 249 & 254 \\
Sludge, 60 mg Pu/L & $\mathrm{Am}$ & 8.3 & 7.0 & 6.6 \\
\hline Acidified & $\mathrm{Ce}$ & 0.7 & 0.6 & 0.5 \\
Supernate & $\mathrm{Cs}$ & $<0.1$ & $<0.1$ & $<0.1$ \\
& $\mathrm{Sr}$ & 0.6 & 0.6 & 0.5 \\
& $\mathrm{Tc}$ & 63 & 63 & 61 \\
& $\mathrm{Y}$ & 0.4 & 0.4 & 0.3 \\
& $\mathrm{Cr}$ & 0.4 & 0.7 & 0.7 \\
& $\mathrm{Co}$ & 1.0 & 0.7 & 0.6 \\
& $\mathrm{Fe}$ & $<0.1$ & $<0.1$ & $<0.1$ \\
& $\mathrm{Mn}$ & 1.0 & 1.0 & 0.9 \\
& $\mathrm{Zn}$ & 2.6 & 2.8 & 2.5 \\
& $\mathrm{Zr}$ & 0.4 & 0.3 & 0.3 \\
& $\mathrm{U}$ & 1.8 & 1.7 & 1.7 \\
& $\mathrm{Pu}$ & 1.5 & 0.9 & 0.8 \\
& $\mathrm{Am}$ & 1.8 & 1.8 & 1.7 \\
\hline \hline
\end{tabular}


d. JSK-2 Anion Exchange Resin. This resin sorbs plutonium well from acid-dissolved sludge solution and also sorbs cerium, technetium, uranium, and americium, but at lower levels. It sorbs only technetium at a useful level from acidified supernate solution. This resin could not be studied with alkaline supernate solution because it is unstable in strongly basic solution.

\begin{tabular}{|c|c|c|c|c|}
\hline \multirow[b]{2}{*}{ Solution } & \multirow[b]{2}{*}{ Absorber } & \multicolumn{3}{|c|}{ Kd Value for Specified Time } \\
\hline & & $30 \mathrm{~min}$ & $2 \mathrm{~h}$ & $6 \mathrm{~h}$ \\
\hline $\begin{array}{l}\text { Acid-Dissolved } \\
\text { Sludge, } 3 \text { g Pu/L }\end{array}$ & $\begin{array}{l}\mathrm{Ce} \\
\mathrm{Cs} \\
\mathrm{Sr} \\
\mathrm{Tc} \\
\mathrm{Y} \\
\mathrm{Cr} \\
\mathrm{Co} \\
\mathrm{Fe} \\
\mathrm{Mn} \\
\mathrm{Zn} \\
\mathrm{Zr} \\
\mathrm{U} \\
\mathrm{Pu} \\
\mathrm{Am}\end{array}$ & $\begin{array}{r}27 \\
0.3 \\
0.6 \\
28 \\
1.6 \\
0.4 \\
<0.1 \\
<0.1 \\
0.3 \\
2.8 \\
5.5 \\
19 \\
142 \\
8.4\end{array}$ & $\begin{array}{r}26 \\
0.1 \\
0.5 \\
28 \\
1.7 \\
0.4 \\
<0.1 \\
<0.1 \\
0.1 \\
1.9 \\
5.6 \\
19 \\
278 \\
7.9\end{array}$ & $\begin{array}{r}24 \\
0.1 \\
0.6 \\
27 \\
1.4 \\
0.5 \\
0.1 \\
0.3 \\
0.1 \\
1.1 \\
5.8 \\
19 \\
422 \\
7.6\end{array}$ \\
\hline $\begin{array}{l}\text { Acid-Dissolved } \\
\text { Sludge, } 60 \mathrm{mg} / \mathrm{L}\end{array}$ & $\begin{array}{l}\mathrm{U} \\
\mathrm{Pu} \\
\mathrm{Am}\end{array}$ & $\begin{array}{r}20 \\
329 \\
9.4 \\
\end{array}$ & $\begin{array}{r}17 \\
337 \\
7.7 \\
\end{array}$ & $\begin{array}{r}17 \\
261 \\
7.2 \\
\end{array}$ \\
\hline $\begin{array}{l}\text { Acidified } \\
\text { Supernate }\end{array}$ & $\begin{array}{l}\mathrm{Ce} \\
\mathrm{Cs} \\
\mathrm{Sr} \\
\mathrm{Tc} \\
\mathrm{Y} \\
\mathrm{Cr} \\
\mathrm{Co} \\
\mathrm{Fe} \\
\mathrm{Mn} \\
\mathrm{Zn} \\
\mathrm{Zr} \\
\mathrm{U} \\
\mathrm{Pu} \\
\mathrm{Am}\end{array}$ & $\begin{array}{r}0.6 \\
0.1 \\
0.2 \\
50 \\
<0.1 \\
<0.1 \\
0.1 \\
<0.1 \\
0.7 \\
1.9 \\
<0.1 \\
1.3 \\
0.2 \\
1.8\end{array}$ & $\begin{array}{c}\text { not measured } \\
\text { not measured } \\
\text { not measured } \\
\text { not measured } \\
\text { not measured } \\
\text { not measured } \\
\text { not measured } \\
0.1 \\
0.2 \\
1.8 \\
\text { not measured } \\
1.2 \\
0.3 \\
1.2 \\
\end{array}$ & $\begin{array}{r}1.2 \\
0.3 \\
0.5 \\
51 \\
0.5 \\
0.6 \\
0.4 \\
0.3 \\
0.3 \\
1.8 \\
0.5 \\
1.2 \\
0.1 \\
1.1\end{array}$ \\
\hline
\end{tabular}


e. JSK-3 Anion Exchange Resin. This resin sorbs plutonium from acid-dissolved sludge solution significantly better than any other absorber, whereas its sorption of other elements from both acidic solutions is similar to that of JSK-2 resin. The exceptionally high sorption of plutonium from acid-dissolved sludge solution exceeds that of the next best absorber, Reillex ${ }^{\mathrm{TM}} \mathrm{HPQ}$ resin, by an order of magnitude. This resin could not be studied with alkaline supernate solution because it is unstable in strongly basic solution.

Table 41. JSK-3 Anion Exchange Resin: Distribution of 14 Elements from Two Simulant Solutions for Hanford Tank 102-SY

\begin{tabular}{|c|c|c|c|c|}
\hline \multirow[b]{2}{*}{ Solution } & \multirow[b]{2}{*}{ Element } & \multicolumn{3}{|c|}{ Kd Value for Specified Time } \\
\hline & & $30 \mathrm{~min}$ & $2 \mathrm{~h}$ & $6 \mathrm{~h}$ \\
\hline Acid-Dissolved & $\overline{\mathrm{Ce}}$ & 23 & 23 & 22 \\
\hline \multirow{13}{*}{ Sludge, $3 \mathrm{G} \mathrm{Pu} / \mathrm{L}$} & Cs & 0.4 & 0.1 & 0.4 \\
\hline & $\mathrm{Sr}$ & 1.0 & 1.3 & 1.3 \\
\hline & Tc & 24 & 24 & 22 \\
\hline & $\mathrm{Y}$ & 1.2 & 1.1 & 1.3 \\
\hline & $\mathrm{Cr}$ & 0.4 & 0.6 & 0.7 \\
\hline & Co & $<0.1$ & 0.3 & 0.1 \\
\hline & $\mathrm{Fe}$ & 0.4 & 0.1 & 0.3 \\
\hline & $\mathrm{Mn}$ & $<0.1$ & 0.2 & 0.4 \\
\hline & $\mathrm{Zn}$ & 1.7 & 1.3 & 0.7 \\
\hline & $\mathrm{Zr}$ & 3.7 & 3.3 & 3.0 \\
\hline & $\mathbf{U}$ & 18 & 18 & 17 \\
\hline & $\mathrm{Pu}$ & 1053 & 1035 & 1173 \\
\hline & Am & 6.2 & 5.3 & 5.0 \\
\hline Acid-Dissolved & $\mathrm{U}$ & 18 & 17 & 17 \\
\hline \multirow{2}{*}{ Sludge, $60 \mathrm{mg} \mathrm{Pu} / \mathrm{L}$} & $\mathrm{Pu}$ & 870 & 1650 & 2185 \\
\hline & $\mathrm{Am}$ & 7.9 & 6.3 & 6.0 \\
\hline Acidified & $\mathrm{Ce}$ & 1.4 & 1.4 & 1.4 \\
\hline \multirow[t]{13}{*}{ Supernate } & Cs & $<0.1$ & $<0.1$ & 0.1 \\
\hline & $\mathrm{Sr}$ & 0.8 & 0.8 & 0.8 \\
\hline & $\mathrm{Tc}$ & 48 & 49 & 50 \\
\hline & $\mathrm{Y}$ & 0.6 & 0.7 & 0.5 \\
\hline & $\mathrm{Cr}$ & 0.6 & 0.5 & 0.6 \\
\hline & Co & 1.1 & 1.3 & 0.9 \\
\hline & $\mathrm{Fe}$ & $<0.1$ & $<0.1$ & $<0.1$ \\
\hline & $\mathrm{Mn}$ & 0.8 & 0.9 & 0.5 \\
\hline & $\mathrm{Zn}$ & 1.7 & 1.3 & 1.5 \\
\hline & $\mathrm{Zr}$ & 0.3 & 0.2 & 0.1 \\
\hline & $\mathrm{U}$ & 2.0 & 1.4 & 1.5 \\
\hline & $\mathrm{Pu}$ & 0.7 & 0.4 & 0.4 \\
\hline & $\mathrm{Am}$ & 1.7 & 1.3 & 1.1 \\
\hline
\end{tabular}


f. Crypt-DER Polymer Resin. Crypt-DER polymer resin is a novel absorber prepared by the Materials Technology: Polymers and Coatings Group at Los Alamos National Laboratory. The granular solid is obtained by polymerizing an octaazacryptand compound with a commercially available di-epoxide resin(DER). The cryptand and functionality contained in the polymer selectively sorbs certain metal ions, such as $\mathrm{Hg}(\mathrm{II})$ and $\mathrm{Cd}(\mathrm{II})$, from solutions having a $\mathrm{pH}$ above 3 . However, when the amine nitrogens of the cryptand are protonated at a lower $\mathrm{pH}$, the cationic functionality binds fluoride ions quite selectively. We included the Crypt-DER polymer resin in this study to obtain additional information about its metal-sorption properties.

It sorbs plutonium, technetium, and uranium at useful levels from acid-dissolved sludge solution, whereas it sorbs cerium and zirconium weakly. It sorbs only technetium at useful $\mathrm{Kd}$ values from acidified supernate solution. Technetium, zinc, and strontium are all sorbed with double-digit Kd values from alkaline supernate solution.

\begin{tabular}{|c|c|c|c|c|}
\hline \multirow[b]{2}{*}{ Solution } & \multirow[b]{2}{*}{ Absorber } & \multicolumn{3}{|c|}{ Kd Value for Specified Time } \\
\hline & & $30 \mathrm{~min}$ & $2 \mathrm{~h}$ & $6 \mathrm{~h}$ \\
\hline $\begin{array}{l}\text { Acid-Dissolved } \\
\text { Sludge }\end{array}$ & $\begin{array}{l}\mathrm{Ce} \\
\mathrm{Cs} \\
\mathrm{Sr} \\
\mathrm{Tc} \\
\mathrm{Y} \\
\mathrm{Cr} \\
\mathrm{Co} \\
\mathrm{Fe} \\
\mathrm{Mn} \\
\mathrm{Zn} \\
\mathrm{Zr} \\
\mathrm{U} \\
\mathrm{Pu} \\
\mathrm{Am}\end{array}$ & $\begin{array}{r}3.0 \\
0.1 \\
0.8 \\
9.8 \\
1.0 \\
<0.1 \\
0.3 \\
<0.1 \\
0.8 \\
0.7 \\
3.2 \\
3.6 \\
4.1 \\
1.0\end{array}$ & $\begin{array}{r}2.9 \\
0.3 \\
0.5 \\
9.9 \\
0.7 \\
<0.1 \\
0.3 \\
<0.1 \\
0.7 \\
0.5 \\
3.6 \\
6.3 \\
8.0 \\
0.9 \\
\end{array}$ & $\begin{array}{r}2.8 \\
0.2 \\
0.4 \\
9.8 \\
0.6 \\
<0.1 \\
0.3 \\
0.1 \\
0.4 \\
0.3 \\
3.9 \\
8.3 \\
19 \\
1.0\end{array}$ \\
\hline $\begin{array}{l}\text { Acidified } \\
\text { Supernate }\end{array}$ & $\begin{array}{l}\mathrm{Ce} \\
\mathrm{Cs} \\
\mathrm{Sr} \\
\mathrm{Tc} \\
\mathrm{Y} \\
\mathrm{Cr} \\
\mathrm{Co} \\
\mathrm{Fe} \\
\mathrm{Mn} \\
\mathrm{Zn} \\
\mathrm{Zr} \\
\mathrm{U} \\
\mathrm{Pu} \\
\mathrm{Am}\end{array}$ & $\begin{array}{r}0.9 \\
0.8 \\
0.7 \\
25 \\
1.0 \\
0.5 \\
2.1 \\
0.1 \\
0.8 \\
1.2 \\
<0.1 \\
1.6 \\
<0.1 \\
1.2 \\
\end{array}$ & $\begin{array}{r}0.7 \\
0.8 \\
0.3 \\
25 \\
0.6 \\
0.1 \\
2.1 \\
0.2 \\
0.8 \\
1.3 \\
0.3 \\
1.7 \\
<0.1 \\
1.4 \\
\end{array}$ & $\begin{array}{r}0.7 \\
1.0 \\
0.4 \\
25 \\
0.8 \\
0.1 \\
2.1 \\
0.4 \\
1.1 \\
1.4 \\
0.2 \\
1.9 \\
<0.1 \\
1.5 \\
\end{array}$ \\
\hline $\begin{array}{l}\text { Alkaline } \\
\text { Supernate }\end{array}$ & $\begin{array}{l}\mathrm{Ce} \\
\mathrm{Cs} \\
\mathrm{Sr} \\
\mathrm{Tc} \\
\mathrm{Y} \\
\mathrm{Cr} \\
\mathrm{Co} \\
\mathrm{Fe} \\
\mathrm{Mn} \\
\mathrm{Zn} \\
\mathrm{Zr} \\
\mathrm{U} \\
\mathrm{Am}\end{array}$ & $\begin{array}{r}5.2 \\
<0.1 \\
3.1 \\
31 \\
6.2 \\
0.2 \\
<0.1 \\
0.6 \\
0.3 \\
4.1 \\
<0.1 \\
<0.1 \\
<0.1\end{array}$ & $\begin{array}{r}6.7 \\
<0.1 \\
12 \\
38 \\
7.5 \\
0.3 \\
<0.1 \\
1.6 \\
0.4 \\
11 \\
0.8 \\
<0.1 \\
<0.1\end{array}$ & $\begin{array}{r}6.4 \\
0.1 \\
15 \\
38 \\
8.5 \\
0.5 \\
<0.1 \\
2.7 \\
0.8 \\
27 \\
1.0 \\
0.3 \\
<0.1\end{array}$ \\
\hline
\end{tabular}


g. PK-01-244 Phosphinic Acid Resin. Professor Spiro Alexandratos at the University of Tennessee, who prepared the PK-01-244 phosphinic acid cation exchanger, advised us that its water content was $45 \%$ and it should not be dried. For this absorber only we did not air-dry the resin but took 455 $\mathrm{mg}$ portions that should have contained $250 \mathrm{mg}$ of the dry resin.
Only cesium and technetium exhibit Kd values consistently higher than unity for sorption from acid-dissolved sludge or acidified supernate solutions. Zirconium, strontium, iron, cerium, yttrium, and manganese are sorbed with double-digit Kd values from alkaline supernate solution.

Table 43. PK-01-244 Resin: Distribution of 14 Elements from Three Simulant Solutions for Hanford Tank 102-SY

\begin{tabular}{|c|c|c|c|c|}
\hline \multirow[b]{2}{*}{ Solution } & \multirow[b]{2}{*}{ Element } & \multicolumn{3}{|c|}{ Kd Value for Specified Time } \\
\hline & & $30 \mathrm{~min}$ & $2 \mathrm{~h}$ & $6 \mathrm{~h}$ \\
\hline Acid-Dissolved & $\mathrm{Ce}$ & 0.8 & 0.8 & 0.5 \\
\hline \multirow[t]{13}{*}{ Sludge } & $\mathrm{Cs}$ & 1.0 & 0.9 & 0.5 \\
\hline & $\mathrm{Sr}$ & 0.6 & 0.6 & 0.5 \\
\hline & $\mathrm{Tc}$ & 1.5 & 1.7 & 1.9 \\
\hline & $\mathrm{Y}$ & 0.6 & 0.8 & 0.6 \\
\hline & $\mathrm{Cr}$ & 0.6 & 0.6 & 0.4 \\
\hline & $\mathrm{Co}$ & 0.9 & 0.7 & 0.5 \\
\hline & $\mathrm{Fe}$ & 0.7 & 0.2 & 0.2 \\
\hline & $\mathrm{Mn}$ & 1.0 & 0.3 & 0.3 \\
\hline & $\mathrm{Zn}$ & 0.8 & 0.4 & 0.4 \\
\hline & $\mathrm{Zr}$ & 1.1 & 0.6 & 1.0 \\
\hline & $\mathrm{U}$ & $<0.1$ & 0.3 & 0.3 \\
\hline & $\mathrm{Pu}$ & 0.6 & 0.3 & 0.2 \\
\hline & $\mathrm{Am}$ & 0.8 & 0.4 & 0.3 \\
\hline Acidified & $\mathrm{Ce}$ & 0.5 & 0.5 & 0.8 \\
\hline \multirow[t]{13}{*}{ Supernate } & Cs & 1.4 & 1.4 & 1.7 \\
\hline & $\mathrm{Sr}$ & 0.3 & 0.3 & 0.7 \\
\hline & $\mathrm{Tc}$ & 0.9 & 0.8 & 1.4 \\
\hline & $\mathrm{Y}$ & 0.7 & 0.4 & 0.7 \\
\hline & $\mathrm{Cr}$ & $<0.1$ & 0.1 & 0.1 \\
\hline & Co & 0.7 & 0.4 & 1.0 \\
\hline & $\mathrm{Fe}$ & 0.2 & 0.1 & 0.1 \\
\hline & $\mathrm{Mn}$ & 0.5 & 0.3 & 0.3 \\
\hline & $\mathrm{Zn}$ & 0.2 & 0.2 & 0.2 \\
\hline & $\mathrm{Zr}$ & 0.5 & 0.1 & 0.1 \\
\hline & $\mathrm{U}$ & 0.5 & 0.4 & 0.5 \\
\hline & $\mathrm{Pu}$ & 0.3 & 0.3 & 0.2 \\
\hline & $\mathrm{Am}$ & $<0.1$ & $<0.1$ & 0.1 \\
\hline Alkaline & $\mathrm{Ce}$ & 10 & 16 & 7.2 \\
\hline \multirow[t]{12}{*}{ Supernate } & Cs & 2.1 & 1.9 & 1.8 \\
\hline & $\mathrm{Sr}$ & 33 & 31 & 19 \\
\hline & $\mathrm{Tc}$ & 1.8 & 1.6 & 1.7 \\
\hline & $\mathrm{Y}$ & 11 & 13 & 7.0 \\
\hline & $\mathrm{Cr}$ & 0.1 & 0.2 & 0.2 \\
\hline & Co & $<0.1$ & $<0.1$ & $<0.1$ \\
\hline & $\mathrm{Fe}$ & 8.3 & 15 & 19 \\
\hline & $\mathrm{Mn}$ & 3.7 & 10 & 13 \\
\hline & $\mathrm{Zn}$ & 1.3 & 2.0 & 2.8 \\
\hline & $\mathrm{Zr}$ & 86 & 90 & 98 \\
\hline & $\mathrm{U}$ & $<0.1$ & 0.5 & 1.1 \\
\hline & $\mathrm{Am}$ & 2.1 & 5.5 & 8.9 \\
\hline
\end{tabular}


3. Polyacrylonitrile (PAN) Composite Absorbers. Although inorganic ion exchangers are known to offer high selectivity, high capacity, and rapid kinetics, their granular and mechanical properties often make them unsuitable for column applications. One way to circumvent these limitations is to incorporate the inorganic exchange materials in beads of a suitable porous polymer. ${ }^{13}$

Nine composite absorbers prepared for us by Dr. Ferdinand Sebesta of the Czech Technical University in Prague consist of various inorganic exchange materials dispersed in modified-binding-polymer beads of polyacrylonitrile (PAN).
Although Dr. Sebesta asked that these composites be kept wet because their performance would be seriously degraded if they were dried, we chose to air-dry them to put them on an equal basis with the other absorbers. We therefore note that the excellent performance obtained with many PAN composites might be even better had we followed Dr. Sebesta's instructions. In particular, the frequently observed slow sorption kinetics might be faster. 
a. TiO-PAN Titanium Dioxide Composite. The titanium dioxide content of the dry TiO-PAN composite was $85 \%$. The air-dried weight of this composite, per water-wet milliliter, was $0.231 \mathrm{~g}$.

TiO-PAN composite sorbs zirconium strongly from acid-dissolved sludge solution, albeit with slow kinetics; only plutonium offers even weak competitive sorption from this solution. It sorbs uranium strongly from acidified supernate solution and uranium, zinc, iron, manganese, strontium, zirconium, and americium strongly from alkaline supernate solution.

Table 44. TiO-PAN Composite: Distribution of 14 Elements from Three Simulant Solutions for Hanford Tank 102-SY

\begin{tabular}{|c|c|c|c|c|}
\hline \multirow[b]{2}{*}{ Solution } & \multirow[b]{2}{*}{ Element } & \multicolumn{3}{|c|}{ Kd Value for Specified Time } \\
\hline & & $30 \mathrm{~min}$ & $2 \mathrm{~h}$ & $6 \mathrm{~h}$ \\
\hline \multirow{14}{*}{$\begin{array}{l}\text { Acid-Dissolved } \\
\text { Sludge }\end{array}$} & $\mathrm{Ce}$ & 0.7 & 0.4 & 0.5 \\
\hline & Cs & 0.3 & 0.1 & 0.4 \\
\hline & $\mathrm{Sr}$ & 1.1 & 0.4 & 0.8 \\
\hline & Tc & 1.0 & 0.7 & 0.9 \\
\hline & $\mathrm{Y}$ & 0.8 & 0.6 & 0.6 \\
\hline & $\mathrm{Cr}$ & 0.5 & 0.4 & 0.5 \\
\hline & Co & 0.6 & 0.3 & 0.4 \\
\hline & $\mathrm{Fe}$ & 0.4 & 0.4 & 0.8 \\
\hline & $\mathrm{Mn}$ & 0.1 & 0.1 & 0.2 \\
\hline & $\mathrm{Zn}$ & $<0.1$ & 0.2 & 0.3 \\
\hline & $\mathrm{Zr}$ & 51 & 323 & 899 \\
\hline & $\mathrm{U}$ & 0.3 & 0.3 & 0.6 \\
\hline & $\mathrm{Pu}$ & 6.3 & 7.7 & 8.6 \\
\hline & $\mathrm{Am}$ & 0.1 & $<0.1$ & $<0.1$ \\
\hline \multirow{14}{*}{$\begin{array}{l}\text { Acidified } \\
\text { Supernate }\end{array}$} & $\mathrm{Ce}$ & 1.6 & 1.9 & 1.6 \\
\hline & Cs & 0.6 & 1.0 & 0.6 \\
\hline & $\mathrm{Sr}$ & 0.2 & 0.5 & 0.4 \\
\hline & Tc & 1.3 & 1.5 & 1.6 \\
\hline & $\mathrm{Y}$ & 1.5 & 2.0 & 1.8 \\
\hline & $\mathrm{Cr}$ & 4.0 & 4.4 & 5.4 \\
\hline & Co & $<0.1$ & $<0.1$ & $<0.1$ \\
\hline & $\mathrm{Fe}$ & 1.4 & 3.8 & 5.2 \\
\hline & $\mathrm{Mn}$ & $<0.1$ & $<0.1$ & 0.1 \\
\hline & $\mathrm{Zn}$ & $<0.1$ & 0.2 & 0.1 \\
\hline & $\mathrm{Zr}$ & 1.5 & 2.0 & 3.2 \\
\hline & $\mathrm{U}$ & 67 & 115 & 185 \\
\hline & $\mathrm{Pu}$ & 1.1 & 1.5 & 2.0 \\
\hline & $\mathrm{Am}$ & 2.1 & 2.8 & 3.2 \\
\hline Alkaline & $\mathrm{Ce}$ & 4.6 & 13 & 57 \\
\hline \multirow{12}{*}{ Supernate } & Cs & 0.5 & 0.5 & 0.4 \\
\hline & $\mathrm{Sr}$ & 917 & 1627 & 2856 \\
\hline & Tc & 0.8 & 0.7 & 0.8 \\
\hline & $\mathbf{Y}$ & 39 & 64 & 102 \\
\hline & $\mathrm{Cr}$ & $<0.1$ & $<0.1$ & 0.1 \\
\hline & $\mathrm{Co}$ & 1.4 & 1.5 & 1.6 \\
\hline & $\mathrm{Fe}$ & 2368 & 4447 & 3523 \\
\hline & Mn & 774 & 1859 & 3110 \\
\hline & $\mathrm{Zn}$ & 2875 & $>6 \mathrm{~K}$ & $>9 \mathrm{~K}$ \\
\hline & $\mathrm{Zr}$ & 615 & 1187 & 2092 \\
\hline & $\mathrm{U}$ & 2350 & $>6 \mathrm{~K}$ & $>9 \mathrm{~K}$ \\
\hline & Am & 109 & 490 & 750 \\
\hline
\end{tabular}


b. NiFC-PAN Nickel Ferrocyanide Composite. The nickel ferrocyanide content of the dry NiFC-PAN composite was $85 \%$. The air-dried weight of this composite, per waterwet milliliter, was $0.231 \mathrm{~g}$.

NiFC-PAN appears to be selective for sorbing cesium from acid-dissolved sludge solution. From acidified supernate solution, it sorbs cesium strongly, zinc well, and cobalt moderately. From alkaline supernate solution, it sorbs zinc, iron, strontium, cesium, zirconium, manganese, and americium very well. We do not understand why the sorption of cesium decreases with increasing time, whereas the sorption of all other elements on NiFC-PAN increases with time.

\begin{tabular}{|c|c|c|c|c|}
\hline \multirow[b]{2}{*}{ Solution } & \multirow[b]{2}{*}{ Element } & \multicolumn{3}{|c|}{ Kd Value for Specified Time } \\
\hline & & $30 \mathrm{~min}$ & $2 \mathrm{~h}$ & $6 \mathrm{~h}$ \\
\hline $\begin{array}{l}\text { Acid-Dissolved } \\
\text { Sludge }\end{array}$ & $\begin{array}{l}\mathrm{Ce} \\
\mathrm{Cs} \\
\mathrm{Sr} \\
\mathrm{Tc} \\
\mathrm{Y} \\
\mathrm{Cr} \\
\mathrm{Co} \\
\mathrm{Fe} \\
\mathrm{Mn} \\
\mathrm{Zn} \\
\mathrm{Zr} \\
\mathrm{U} \\
\mathrm{Pu} \\
\mathrm{Am}\end{array}$ & $\begin{array}{r}0.5 \\
45 \\
0.5 \\
0.4 \\
0.5 \\
0.5 \\
1.1 \\
0.4 \\
0.3 \\
0.3 \\
1.0 \\
0.6 \\
0.2 \\
<0.1\end{array}$ & $\begin{array}{r}0.3 \\
49 \\
0.6 \\
0.8 \\
0.7 \\
0.5 \\
1.1 \\
0.3 \\
0.3 \\
0.4 \\
1.1 \\
0.4 \\
0.7 \\
<0.1 \\
\end{array}$ & $\begin{array}{l}0.5 \\
49 \\
0.7 \\
0.7 \\
0.6 \\
0.6 \\
1.1 \\
0.5 \\
0.4 \\
0.4 \\
1.7 \\
0.2 \\
0.4 \\
0.1 \\
\end{array}$ \\
\hline $\begin{array}{l}\text { Acidified } \\
\text { Supernate }\end{array}$ & $\begin{array}{l}\mathrm{Ce} \\
\mathrm{Cs} \\
\mathrm{Sr} \\
\mathrm{Tc} \\
\mathrm{Y} \\
\mathrm{Cr} \\
\mathrm{Co} \\
\mathrm{Fe} \\
\mathrm{Mn} \\
\mathrm{Zn} \\
\mathrm{Zr} \\
\mathrm{U} \\
\mathrm{Pu} \\
\mathrm{Am}\end{array}$ & $\begin{array}{r}0.3 \\
3533 \\
<0.1 \\
1.0 \\
<0.1 \\
0.9 \\
8.9 \\
1.4 \\
0.5 \\
61 \\
0.5 \\
2.1 \\
<0.1 \\
0.4\end{array}$ & $\begin{array}{r}0.3 \\
>11 \mathrm{~K} \\
<0.1 \\
0.7 \\
0.2 \\
0.8 \\
10 \\
1.7 \\
0.7 \\
94 \\
0.5 \\
2.2 \\
<0.1 \\
0.3\end{array}$ & $\begin{array}{r}0.2 \\
>6 \mathrm{~K} \\
<0.1 \\
0.8 \\
0.2 \\
1.2 \\
11 \\
2.3 \\
0.5 \\
90 \\
0.7 \\
2.0 \\
0.2 \\
0.2 \\
\end{array}$ \\
\hline $\begin{array}{l}\text { Alkaline } \\
\text { Supernate }\end{array}$ & $\begin{array}{l}\mathrm{Ce} \\
\mathrm{Cs} \\
\mathrm{Sr} \\
\mathrm{Tc} \\
\mathrm{Y} \\
\mathrm{Cr} \\
\mathrm{Co} \\
\mathrm{Fe} \\
\mathrm{Mn} \\
\mathrm{Zn} \\
\mathrm{Zr} \\
\mathrm{U} \\
\mathrm{Am}\end{array}$ & $\begin{array}{r}2.6 \\
2090 \\
1121 \\
0.2 \\
48 \\
1.1 \\
5.7 \\
1524 \\
59 \\
3800 \\
179 \\
7.0 \\
65\end{array}$ & $\begin{array}{r}4.9 \\
943 \\
3736 \\
0.4 \\
72 \\
1.3 \\
7.0 \\
3676 \\
167 \\
>11 \mathrm{~K} \\
375 \\
8.9 \\
189\end{array}$ & $\begin{array}{r}6.4 \\
76 \\
4651 \\
1.6 \\
99 \\
2.5 \\
8.4 \\
>6 \mathrm{~K} \\
363 \\
>17 \mathrm{~K} \\
474 \\
9.0 \\
407\end{array}$ \\
\hline
\end{tabular}


c. MgO-PAN Magnesium Oxide Composite. The magnesium oxide content of the dry MgO-PAN composite was $80 \%$. The air-dried weight of this composite, per waterwet milliliter, was $0.226 \mathrm{~g}$.

MgO-PAN sorbs zirconium and several other elements slightly from acid-dissolved sludge solution. This composite sorbs zinc, iron, strontium, zirconium, manganese, and americium well from alkaline supernate solution.

Because this absorber was unstable in acidified supernate solution, we were unable to obtain $\mathrm{Kd}$ values for sorption from that solution. Resin swelling and solution discoloration indicated some instability in all solutions.

\begin{tabular}{|c|c|c|c|c|}
\hline \multicolumn{5}{|c|}{$\begin{array}{l}\text { Table 46. MgO-PAN Composite: Distribution of } 14 \text { Elements from } \\
\text { Two Simulant Solutions for Hanford Tank 102-SY }\end{array}$} \\
\hline \multirow{2}{*}{ Solution } & \multirow[b]{2}{*}{ Element } & \multicolumn{3}{|c|}{ Kd Value for Specified Time } \\
\hline & & $30 \mathrm{~min}$ & $2 \mathrm{~h}$ & $6 \mathrm{~h}$ \\
\hline$\overline{\text { Acid-Dissolved }}$ & $\mathrm{Ce}$ & 1.5 & 1.5 & 1.5 \\
\hline \multirow[t]{13}{*}{ Sludge } & Cs & 0.8 & 1.1 & 1.1 \\
\hline & $\mathrm{Sr}$ & 1.4 & 1.6 & 2.0 \\
\hline & Tc & 1.4 & 1.7 & 1.9 \\
\hline & $\mathrm{Y}$ & 1.2 & 1.5 & 1.5 \\
\hline & $\mathrm{Cr}$ & 1.5 & 1.4 & 1.6 \\
\hline & Co & 1.3 & 1.4 & 1.4 \\
\hline & $\mathrm{Fe}$ & 0.7 & 0.8 & 1.0 \\
\hline & $\mathrm{Mn}$ & 0.3 & 0.3 & 0.3 \\
\hline & $\mathrm{Zn}$ & 0.4 & 0.4 & 0.4 \\
\hline & $\mathrm{Zr}$ & 2.2 & 3.2 & 4.7 \\
\hline & $\mathrm{U}$ & 0.1 & 0.3 & 0.5 \\
\hline & $\mathrm{Pu}$ & 0.9 & 1.3 & 1.6 \\
\hline & $\mathrm{Am}$ & 0.3 & 0.3 & 0.3 \\
\hline Alkaline & $\mathrm{Ce}$ & 11 & 27 & 25 \\
\hline \multirow[t]{12}{*}{ Supernate } & Cs & $<0.1$ & $<0.1$ & $<0.1$ \\
\hline & $\mathrm{Sr}$ & 499 & 768 & 689 \\
\hline & $\mathrm{Tc}$ & 1.3 & 1.3 & 1.6 \\
\hline & $\mathrm{Y}$ & 35 & 58 & 98 \\
\hline & $\mathrm{Cr}$ & 0.2 & 0.1 & 0.4 \\
\hline & $\mathrm{Co}$ & 1.0 & 1.0 & 1.1 \\
\hline & $\mathrm{Fe}$ & 754 & 1620 & 2048 \\
\hline & $\mathrm{Mn}$ & 172 & 373 & 623 \\
\hline & $\mathrm{Zn}$ & 2051 & 4193 & 4040 \\
\hline & $\mathrm{Zr}$ & 233 & 460 & 643 \\
\hline & $\mathrm{U}$ & 4.0 & 6.4 & 9.5 \\
\hline & Am & 10 & 46 & 121 \\
\hline
\end{tabular}

d. MnO-PAN Manganese Dioxide Composite. The manganese dioxide content of the dry MnO-PAN composite was $85 \%$. The air-dried weight of this composite, per waterwet milliliter, was $0.210 \mathrm{~g}$.

MnO-PAN sorbs zirconium and plutonium somewhat from acid-dissolved sludge solution. Cerium and americium show an order-of-magnitude higher sorption than yttrium from acidified supernate solution. Strontium, zinc, zirconium, cerium, iron, and yttrium all sorb well from alkaline supernate solution.
Kd values for several elements for the 30-min contact period are much lower than $\mathrm{Kd}$ values for the 2-h and 6-h contact periods. We believe these differences are a result of the filtration of most $2-\mathrm{h}$ and 6-h postcontact solutions, but not the 30 -min postcontact solutions, through a $0.45-\mu \mathrm{m}$ filter before the gamma spectrometric assay. 
Table 47. MnO-PAN Composite: Distribution of 14 Elements from Three Simulant Solutions for Hanford Tank 102-SY

\begin{tabular}{|c|c|c|c|c|}
\hline \multirow[b]{2}{*}{ Solution } & \multirow[b]{2}{*}{ Element } & \multicolumn{3}{|c|}{ Kd Value for Specified Time } \\
\hline & & $30 \mathrm{~min}$ & $2 \mathrm{~h}$ & $6 \mathrm{~h}$ \\
\hline Acid-Dissolved & $\mathrm{Ce}$ & 1.1 & 1.0 & 0.9 \\
\hline \multirow{13}{*}{ Sludge } & Cs & 1.2 & 1.2 & 1.1 \\
\hline & $\mathrm{Sr}$ & 0.9 & 0.6 & 0.6 \\
\hline & $\mathrm{Tc}$ & 1.2 & 1.3 & 1.0 \\
\hline & $\mathrm{Y}$ & 1.1 & 1.1 & 0.8 \\
\hline & $\mathrm{Cr}$ & 0.6 & 0.9 & 0.6 \\
\hline & Co & 0.9 & 0.8 & 0.7 \\
\hline & $\mathrm{Fe}$ & 0.7 & 1.2 & 1.0 \\
\hline & $\mathrm{Mn}$ & 0.3 & 0.3 & 0.5 \\
\hline & $\mathrm{Zn}$ & $<0.1$ & 0.2 & 0.2 \\
\hline & $\mathrm{Zr}$ & 4.0 & 6.0 & 6.8 \\
\hline & $\mathbf{U}$ & 0.5 & 0.1 & 0.5 \\
\hline & $\mathrm{Pu}$ & 2.5 & 2.7 & 2.8 \\
\hline & Am & 0.2 & 0.2 & 0.1 \\
\hline \multirow{14}{*}{$\begin{array}{l}\text { Acidified } \\
\text { Supernate }\end{array}$} & $\mathrm{Ce}$ & 24 & 25 & 26 \\
\hline & $\mathrm{Cs}$ & 0.5 & 0.8 & 1.1 \\
\hline & $\mathrm{Sr}$ & $<0.1$ & $<0.1$ & $<0.1$ \\
\hline & $\mathrm{Tc}$ & 1.1 & 1.4 & 1.4 \\
\hline & $\mathrm{Y}$ & 2.0 & 2.1 & 1.8 \\
\hline & $\mathrm{Cr}$ & 3.0 & 4.3 & 4.8 \\
\hline & Co & $<0.1$ & 0.6 & 0.9 \\
\hline & $\mathrm{Fe}$ & 3.1 & 4.5 & 5.8 \\
\hline & $\mathrm{Mn}$ & 1.8 & 1.9 & 2.2 \\
\hline & $\mathrm{Zn}$ & $<0.1$ & 0.1 & 0.2 \\
\hline & $\mathrm{Zr}$ & 1.7 & 2.2 & 2.3 \\
\hline & U & 11 & 13 & 13 \\
\hline & $\mathrm{Pu}$ & 2.8 & 3.3 & 3.8 \\
\hline & $\mathrm{Am}$ & 32 & 30 & 26 \\
\hline & $\mathrm{Ce}$ & 5.4 & 1007 & $>1500$ \\
\hline \multirow{12}{*}{ Supernate ${ }^{a}$} & Cs & 0.4 & 0.6 & 0.8 \\
\hline & $\mathrm{Sr}$ & 27 & $>8 \mathrm{~K}$ & $>8 \mathrm{~K}$ \\
\hline & Tc & 1.0 & 2.1 & 2.0 \\
\hline & $\mathrm{Y}$ & 17 & 151 & 229 \\
\hline & $\mathrm{Cr}$ & $<0.1$ & 0.3 & 0.3 \\
\hline & Co & 0.8 & 1.6 & 1.7 \\
\hline & $\mathrm{Fe}$ & 107 & 244 & $>1000$ \\
\hline & Mn & 25 & 76 & $>1000$ \\
\hline & $\mathrm{Zn}$ & 1519 & 3806 & $>10 \mathrm{~K}$ \\
\hline & $\mathrm{Zr}$ & 270 & 925 & 1543 \\
\hline & $\mathrm{U}$ & 12 & 14 & 15 \\
\hline & Am & 21 & 65 & $>1000$ \\
\hline
\end{tabular}

${ }^{a}$ After the absorber/solution contacts for $2 \mathrm{~h}$ and $6 \mathrm{~h}$, the alkaline supernate solutions for cerium, technetium, yttrium, chromium, iron, and zirconium were passed through a $0.45-\mu \mathrm{m}$ filter to remove visible particles. The same was done for cesium, strontium, cobalt, manganese, zinc, uranium, and americium after the 6-h contact. 
e. NaY-PANSynthetic Zeolite Composite. The synthetic zeolite content of the dry NaY-PAN composite was $94 \%$. The air-dried weight of this composite, per water-wet milliliter, was $0.305 \mathrm{~g}$.

NaY-PAN appears to be selective for sorbing zirconium and plutonium from acid-dissolved sludge solution. Only cesium offers double-digit Kd values for sorption from acidified supernate solution. For sorption from alkaline supernate solution, zirconium, yttrium, manganese, strontium, americium, and iron offer double-digit $\mathrm{Kd}$ values.

Table 48. NaY-PAN Composite: Distribution of 14 Elements from Three Simulant Solutions for Hanford Tank 102-SY

\begin{tabular}{|c|c|c|c|c|}
\hline \multirow[b]{2}{*}{ Solution } & \multirow[b]{2}{*}{ Element } & \multicolumn{3}{|c|}{ Kd Value for Specified Time } \\
\hline & & $30 \mathrm{~min}$ & $2 \mathrm{~h}$ & $6 \mathrm{~h}$ \\
\hline \multirow{14}{*}{$\begin{array}{l}\text { Acid-Dissolved } \\
\text { Sludge }\end{array}$} & $\mathrm{Ce}$ & 0.9 & 1.1 & 0.9 \\
\hline & Cs & 0.9 & 1.0 & 0.7 \\
\hline & $\mathrm{Sr}$ & 0.7 & 0.9 & 0.9 \\
\hline & Tc & 1.0 & 0.9 & 0.9 \\
\hline & $\mathrm{Y}$ & 0.9 & 1.0 & 0.9 \\
\hline & $\mathrm{Cr}$ & 0.9 & 1.0 & 0.9 \\
\hline & Co & 0.8 & 0.9 & 0.9 \\
\hline & $\mathrm{Fe}$ & 2.2 & 1.6 & 1.5 \\
\hline & $\mathrm{Mn}$ & 0.4 & 0.1 & 0.1 \\
\hline & $\mathrm{Zn}$ & 0.2 & 0.2 & 0.1 \\
\hline & $\mathrm{Zr}$ & 107 & 191 & 198 \\
\hline & $\mathrm{U}$ & 0.3 & 0.1 & 0.3 \\
\hline & $\mathrm{Pu}$ & 12 & 15 & 15 \\
\hline & $\mathrm{Am}$ & $<0.1$ & $<0.1$ & $<0.1$ \\
\hline \multirow{14}{*}{$\begin{array}{l}\text { Acidified } \\
\text { Supernate }\end{array}$} & $\mathrm{Ce}$ & 4.6 & 4.0 & 3.3 \\
\hline & Cs & 11 & 10 & 10 \\
\hline & $\mathrm{Sr}$ & 0.6 & 1.0 & 0.9 \\
\hline & $\mathrm{Tc}$ & 0.1 & 0.5 & 0.3 \\
\hline & $\mathrm{Y}$ & 1.5 & 1.6 & 1.4 \\
\hline & $\mathrm{Cr}$ & 2.3 & 4.1 & 4.8 \\
\hline & Co & 0.7 & 0.4 & 0.4 \\
\hline & $\mathrm{Fe}$ & 0.2 & 0.5 & 0.9 \\
\hline & $\mathbf{M n}$ & 0.8 & 0.7 & 0.7 \\
\hline & $\mathrm{Zn}$ & 1.5 & 1.3 & 1.2 \\
\hline & $\mathrm{Zr}$ & 0.2 & 0.8 & 0.8 \\
\hline & $\mathrm{U}$ & 0.9 & 0.8 & 0.9 \\
\hline & $\mathrm{Pu}$ & $<0.1$ & $<0.1$ & $<0.1$ \\
\hline & $\mathrm{Am}$ & 3.7 & 3.5 & 3.3 \\
\hline Alkaline & $\mathrm{Ce}$ & 1.1 & 1.9 & 3.1 \\
\hline \multirow[t]{12}{*}{ Supernate } & Cs & 8.0 & 7.8 & 7.5 \\
\hline & $\mathrm{Sr}$ & 12 & 15 & 20 \\
\hline & Tc & 0.2 & 0.1 & 0.1 \\
\hline & $\mathrm{Y}$ & 10 & 23 & 40 \\
\hline & $\mathrm{Cr}$ & $<0.1$ & $<0.1$ & $<0.1$ \\
\hline & Co & 0.3 & 0.2 & 0.2 \\
\hline & $\mathrm{Fe}$ & 5.2 & 7.6 & 11 \\
\hline & $\mathrm{Mn}$ & 6.9 & 14 & 28 \\
\hline & $\mathrm{Zn}$ & 2.0 & 2.7 & 3.4 \\
\hline & $\mathrm{Zr}$ & 19 & 34 & 50 \\
\hline & $\mathrm{U}$ & 1.7 & 2.3 & 2.4 \\
\hline & $\mathrm{Am}$ & 1.3 & 12 & 35 \\
\hline
\end{tabular}


f. M315-PAN Synthetic Mordenite Composite. The synthetic mordenite content of the dry M315-PAN composite was $93 \%$. The air-dried weight of this composite, per waterwet milliliter, was $0.222 \mathrm{~g}$.
M315-PAN shows good selectivity for sorbing cesium and zirconium from acid-dissolved sludge solution and for sorbing cesium from acidified supernate solution. It sorbs cesium, strontium, zirconium, cerium, and yttrium from alkaline supernate solution.

\begin{tabular}{|c|c|c|c|c|}
\hline \multirow[b]{2}{*}{ Solution } & \multirow[b]{2}{*}{ Element } & \multicolumn{3}{|c|}{ Kd Value for Specified Time } \\
\hline & & $30 \mathrm{~min}$ & $2 \mathrm{~h}$ & $6 \mathrm{~h}$ \\
\hline $\begin{array}{l}\text { Acid-Dissolved } \\
\text { Sludge }\end{array}$ & $\begin{array}{l}\mathrm{Ce} \\
\mathrm{Cs} \\
\mathrm{Sr} \\
\mathrm{Tc} \\
\mathrm{Y} \\
\mathrm{Cr} \\
\mathrm{Co} \\
\mathrm{Fe} \\
\mathrm{Mn} \\
\mathrm{Zn} \\
\mathrm{Zr} \\
\mathrm{U} \\
\mathrm{Pu} \\
\mathrm{Am}\end{array}$ & $\begin{array}{r}0.9 \\
50 \\
0.8 \\
0.7 \\
1.0 \\
0.8 \\
1.3 \\
0.8 \\
0.3 \\
0.3 \\
27 \\
0.4 \\
1.1 \\
<0.1 \\
\end{array}$ & $\begin{array}{r}1.0 \\
64 \\
1.0 \\
0.7 \\
1.0 \\
1.0 \\
1.0 \\
0.5 \\
0.2 \\
0.4 \\
67 \\
0.6 \\
1.0 \\
<0.1 \\
\end{array}$ & $\begin{array}{r}0.8 \\
61 \\
0.9 \\
0.8 \\
0.8 \\
0.7 \\
1.1 \\
0.5 \\
0.2 \\
0.3 \\
96 \\
0.4 \\
1.2 \\
<0.1 \\
\end{array}$ \\
\hline $\begin{array}{l}\text { Acidified } \\
\text { Supernate }\end{array}$ & $\begin{array}{l}\mathrm{Ce} \\
\mathrm{Cs} \\
\mathrm{Sr} \\
\mathrm{Tc} \\
\mathrm{Y} \\
\mathrm{Cr} \\
\mathrm{Co} \\
\mathrm{Fe} \\
\mathrm{Mn} \\
\mathrm{Zn} \\
\mathrm{Zr} \\
\mathrm{U} \\
\mathrm{Pu} \\
\mathrm{Am}\end{array}$ & $\begin{array}{r}0.5 \\
191 \\
1.1 \\
0.3 \\
0.6 \\
0.6 \\
0.1 \\
0.5 \\
0.6 \\
0.5 \\
<0.1 \\
1.2 \\
<0.1 \\
0.8 \\
\end{array}$ & $\begin{array}{r}0.2 \\
227 \\
1.2 \\
0.6 \\
0.6 \\
0.7 \\
<0.1 \\
0.7 \\
0.6 \\
0.2 \\
0.3 \\
0.7 \\
<0.1 \\
0.6 \\
\end{array}$ & $\begin{array}{r}0.4 \\
231 \\
1.4 \\
0.6 \\
0.7 \\
1.0 \\
<0.1 \\
0.7 \\
0.6 \\
0.4 \\
0.5 \\
0.9 \\
<0.1 \\
0.7 \\
\end{array}$ \\
\hline $\begin{array}{l}\text { Alkaline } \\
\text { Supernate }\end{array}$ & $\begin{array}{l}\mathrm{Ce} \\
\mathrm{Cs} \\
\mathrm{Sr} \\
\mathrm{Tc} \\
\mathrm{Y} \\
\mathrm{Cr} \\
\mathrm{Co} \\
\mathrm{Fe} \\
\mathrm{Mn} \\
\mathrm{Zn} \\
\mathrm{Zr} \\
\mathrm{U} \\
\mathrm{Am}\end{array}$ & $\begin{array}{r}5.3 \\
161 \\
22 \\
0.4 \\
11 \\
<0.1 \\
0.2 \\
1.7 \\
2.9 \\
0.7 \\
12 \\
<0.1 \\
<0.1\end{array}$ & $\begin{array}{r}13 \\
177 \\
165 \\
0.4 \\
31 \\
<0.1 \\
0.1 \\
2.8 \\
4.8 \\
1.2 \\
18 \\
0.6 \\
4.8\end{array}$ & $\begin{array}{r}53 \\
177 \\
432 \\
0.3 \\
67 \\
<0.1 \\
<0.1 \\
3.6 \\
6.1 \\
1.5 \\
21 \\
0.6 \\
8.6\end{array}$ \\
\hline
\end{tabular}


g. NaTiO-PAN Sodium Titanate Composite. The sodium titanate content of the dry NaTiO-PAN composite was $92 \%$. The air-dried weight of this composite, per waterwet milliliter, was $0.324 \mathrm{~g}$.

NaTiO-PAN sorbs zirconium moderately from aciddissolved sludge solution. Only zinc and cesium sorb with double-digit $\mathrm{Kd}$ values from acidified supernate solution. This composite sorbs iron, zinc, zirconium, uranium, manganese, and strontium with at least tripledigit $\mathrm{Kd}$ values, whereas it sorbs americium, cerium, and yttrium at lower values.

Table 50. NaTiO-PAN Composite: Distribution of 14 Elements from Three Simulant Solutions for Hanford Tank 102-SY

\begin{tabular}{|c|c|c|c|c|}
\hline \multirow[b]{2}{*}{ Solution } & \multirow[b]{2}{*}{ Element } & \multicolumn{3}{|c|}{ Kd Value for Specified Time } \\
\hline & & $30 \mathrm{~min}$ & $2 \mathrm{~h}$ & $6 h$ \\
\hline Acid-Dissolved & $\mathrm{Ce}$ & 0.9 & 0.5 & 0.8 \\
\hline \multirow{13}{*}{ Sludge } & Cs & 2.2 & 1.6 & 1.9 \\
\hline & $\mathrm{Sr}$ & 0.5 & 0.6 & 0.7 \\
\hline & $\mathrm{Tc}$ & 0.9 & 0.6 & 1.1 \\
\hline & $\mathrm{Y}$ & 1.2 & 0.7 & 0.9 \\
\hline & $\mathrm{Cr}$ & 0.7 & 0.4 & 0.7 \\
\hline & Co & 0.9 & 0.6 & 0.7 \\
\hline & $\mathrm{Fe}$ & 0.7 & 0.3 & 0.4 \\
\hline & $\mathrm{Mn}$ & 0.4 & 0.4 & 0.4 \\
\hline & $\mathrm{Zn}$ & 0.4 & 0.4 & 0.4 \\
\hline & $\mathrm{Zr}$ & 3.5 & 5.4 & 13 \\
\hline & $\bar{U}$ & 0.2 & 0.7 & 0.7 \\
\hline & $\mathrm{Pu}$ & 0.9 & 0.8 & 1.0 \\
\hline & $\mathrm{Am}$ & $<0.1$ & $<0.1$ & $<0.1$ \\
\hline Acidified & $\mathrm{Ce}$ & 3.7 & 5.2 & 6.6 \\
\hline \multirow{13}{*}{ Supernate } & Cs & 11 & 12 & 12 \\
\hline & $\mathrm{Sr}$ & 4.9 & 5.3 & 5.0 \\
\hline & $\mathrm{Tc}$ & 0.7 & 0.4 & 0.5 \\
\hline & $\mathrm{Y}$ & 3.1 & 3.5 & 4.3 \\
\hline & $\mathrm{Cr}$ & 0.8 & 0.7 & 1.0 \\
\hline & $\mathrm{Co}$ & 6.2 & 7.9 & 9.5 \\
\hline & $\mathrm{Fe}$ & 0.5 & 0.6 & 1.0 \\
\hline & $\mathrm{Mn}$ & 6.0 & 7.3 & 7.6 \\
\hline & $\mathrm{Zn}$ & 21 & 45 & 71 \\
\hline & $\mathrm{Zr}$ & $<0.1$ & $<0.1$ & 0.2 \\
\hline & $\mathrm{U}$ & 4.5 & 6.8 & 8.2 \\
\hline & $\mathrm{Pu}$ & $<0.1$ & $<0.1$ & 0.1 \\
\hline & $\mathrm{Am}$ & 4.6 & 6.0 & 6.9 \\
\hline Alkaline & $\mathrm{Ce}$ & 2.5 & 6.1 & 14 \\
\hline \multirow[t]{12}{*}{ Supernate } & Cs & 1.9 & 1.6 & 1.5 \\
\hline & $\mathrm{Sr}$ & 314 & 410 & 296 \\
\hline & Tc & 0.6 & 0.3 & 0.4 \\
\hline & $\mathrm{Y}$ & 17 & 40 & 68 \\
\hline & $\mathrm{Cr}$ & $<0.1$ & $<0.1$ & 0.1 \\
\hline & Co & 0.2 & $<0.1$ & $<0.1$ \\
\hline & $\mathrm{Fe}$ & 537 & 1350 & 1703 \\
\hline & $\mathrm{Mn}$ & 142 & 319 & 616 \\
\hline & $\mathrm{Zn}$ & 115 & 540 & 1117 \\
\hline & $\mathrm{Zr}$ & 189 & 526 & 833 \\
\hline & $\mathrm{U}$ & 48 & 183 & 403 \\
\hline & $\mathrm{Am}$ & 1.4 & 18 & 55 \\
\hline
\end{tabular}




\section{h. $\mathrm{Ba}(\mathrm{Ca}) \mathrm{SO}_{4} \mathrm{PAN}$ Barium/Calcium Sulfate} Composite. The barium/calcium sulfate content of the dry $\mathrm{Ba}(\mathrm{Ca}) \mathrm{SO}_{4}$-PAN composite was $85 \%$. The air-dried weight of this composite, per water-wet milliliter, was $0.198 \mathrm{~g}$.
$\mathrm{Ba}(\mathrm{Ca}) \mathrm{SO}_{4}$-PAN sorbs no elements at useful levels from acid-dissolved sludge solution. It sorbs strontium and cerium somewhat from acidified supernate solution and sorbs many elements with at least double-digit $\mathrm{Kd}$ values from alkaline supernate solution.

\begin{tabular}{|c|c|c|c|c|}
\hline \multirow[b]{2}{*}{ Solution } & \multirow[b]{2}{*}{ Element } & \multicolumn{3}{|c|}{ Kd Value for Specified Time } \\
\hline & & 30 min & $2 \mathrm{~h}$ & $6 \mathrm{~h}$ \\
\hline \multirow{14}{*}{$\begin{array}{l}\text { Acid-Dissolved } \\
\text { Sludge }\end{array}$} & $\mathrm{Ce}$ & 0.4 & 0.4 & 0.3 \\
\hline & Cs & 1.1 & 1.6 & 1.1 \\
\hline & $\mathrm{Sr}$ & 1.5 & 1.7 & 1.5 \\
\hline & $\mathrm{Tc}$ & 1.0 & 1.1 & 1.0 \\
\hline & $\mathrm{Y}$ & 0.8 & 0.7 & 0.9 \\
\hline & $\mathrm{Cr}$ & 1.1 & 0.9 & 0.9 \\
\hline & Co & 1.0 & 0.9 & 0.8 \\
\hline & $\mathrm{Fe}$ & $<0.1$ & 0.2 & 0.2 \\
\hline & $\mathrm{Mn}$ & 0.3 & 0.1 & 0.1 \\
\hline & $\mathrm{Zn}$ & 0.2 & 0.2 & 0.2 \\
\hline & $\mathrm{Zr}$ & 1.0 & 2.0 & 1.9 \\
\hline & $\mathrm{U}$ & 0.4 & 0.5 & 0.4 \\
\hline & $\mathrm{Pu}$ & 0.9 & 0.9 & 0.7 \\
\hline & $\mathrm{Am}$ & 0.1 & $<0.1$ & 0.1 \\
\hline \multirow{14}{*}{$\begin{array}{l}\text { Acidified } \\
\text { Supernate }\end{array}$} & $\mathrm{Ce}$ & 5.4 & 6.1 & 8.6 \\
\hline & Cs & 0.3 & 0.1 & 0.2 \\
\hline & $\mathrm{Sr}$ & 7.3 & 8.0 & 12 \\
\hline & Tc & 0.6 & 1.0 & 1.2 \\
\hline & $\mathrm{Y}$ & 0.8 & 0.3 & 0.3 \\
\hline & $\mathrm{Cr}$ & $<0.1$ & $<0.1$ & 0.2 \\
\hline & Co & 0.4 & 0.3 & 0.2 \\
\hline & $\mathrm{Fe}$ & 0.5 & 0.7 & 0.9 \\
\hline & $\mathrm{Mn}$ & $<0.1$ & 0.1 & $<0.1$ \\
\hline & $\mathrm{Zn}$ & 0.4 & 0.1 & 0.2 \\
\hline & $\mathrm{Zr}$ & 0.7 & 0.7 & 0.8 \\
\hline & $\mathrm{U}$ & 1.2 & 1.7 & 2.2 \\
\hline & $\mathrm{Pu}$ & 0.8 & 0.5 & 1.0 \\
\hline & Am & 0.8 & 0.6 & 1.3 \\
\hline \multirow{13}{*}{$\begin{array}{l}\text { Alkaline } \\
\text { Supernate }\end{array}$} & $\mathrm{Ce}$ & 5.3 & 12 & 24 \\
\hline & Cs & $<0.1$ & $<0.1$ & $<0.1$ \\
\hline & $\mathrm{Sr}$ & 76 & 116 & 146 \\
\hline & Tc & 0.3 & 0.9 & 1.1 \\
\hline & $Y$ & 16 & 34 & 61 \\
\hline & $\mathrm{Cr}$ & 1.7 & 2.2 & 2.6 \\
\hline & Co & 0.3 & 0.5 & 0.5 \\
\hline & $\mathrm{Fe}$ & 12 & 19 & 25 \\
\hline & $\mathrm{Mn}$ & 17 & 58 & 104 \\
\hline & $\mathrm{Zn}$ & 1.5 & 0.8 & 1.0 \\
\hline & $\mathrm{Zr}$ & 161 & 327 & 482 \\
\hline & $\mathrm{U}$ & 15 & 22 & 37 \\
\hline & $\mathrm{Am}$ & 14 & 71 & 171 \\
\hline
\end{tabular}


i. AMP-PAN Ammonium Molybdophosphate Composite. The ammonium molybdophosphate content of the dry composite was $81 \%$. The air-dried weight of this composite, per water-wet milliliter, was $0.235 \mathrm{~g}$.

AMP-PAN strongly and selectively sorbs cesium from acid-dissolved sludge solution. It also strongly sorbs cesium from acidified supernate solution, but the cesium is accompanied by lanthanides and americium. It sorbs only zinc and iron with double-digit $\mathrm{Kd}$ values from alkaline supernate solution, which suggests that cesium sorbed from acidic solution could be eluted with basic solution.

Table 52. AMP-PAN Composite: Distribution of 14 Elements from Three Simulant Solutions for Hanford Tank 102-SY

\begin{tabular}{|c|c|c|c|c|}
\hline \multirow[b]{2}{*}{ Solution } & \multirow[b]{2}{*}{ Element } & \multicolumn{3}{|c|}{ Kd Value for Specified Time } \\
\hline & & $30 \mathrm{~min}$ & $2 \mathrm{~h}$ & $6 \mathrm{~h}$ \\
\hline Acid-Dissolved & $\mathrm{Ce}$ & 0.4 & 0.2 & 0.4 \\
\hline \multirow{13}{*}{ Sludge } & Cs & 1626 & 4072 & $>5 \mathrm{~K}$ \\
\hline & $\mathrm{Sr}$ & 0.5 & 0.7 & 0.9 \\
\hline & $\mathrm{Tc}$ & 0.8 & 0.9 & 1.2 \\
\hline & $\mathrm{Y}$ & 0.8 & 0.5 & 0.4 \\
\hline & $\mathrm{Cr}$ & 1.0 & 0.9 & 1.0 \\
\hline & Co & 0.8 & 0.8 & 0.8 \\
\hline & $\mathrm{Fe}$ & 0.1 & 0.1 & 0.1 \\
\hline & $\mathrm{Mn}$ & 0.2 & 0.1 & 0.4 \\
\hline & $\mathrm{Zn}$ & 0.2 & 0.1 & 0.2 \\
\hline & $\mathrm{Zr}$ & 1.4 & 2.9 & 4.7 \\
\hline & $\mathrm{U}$ & 0.2 & 0.1 & 0.6 \\
\hline & $\mathrm{Pu}$ & 0.3 & 0.4 & 1.0 \\
\hline & Am & $<0.1$ & $<0.1$ & $<0.1$ \\
\hline Acidified & $\mathrm{Ce}$ & 12 & 22 & 28 \\
\hline \multirow[t]{13}{*}{ Supernate } & Cs & 2133 & 4636 & $>8 \mathrm{~K}$ \\
\hline & $\mathrm{Sr}$ & $<0.1$ & $<0.1$ & $<0.1$ \\
\hline & $\mathrm{Tc}$ & 0.8 & 1.2 & 1.4 \\
\hline & $\mathrm{Y}$ & 15 & 27 & 34 \\
\hline & $\mathrm{Cr}$ & 0.5 & 1.3 & 1.3 \\
\hline & Co & 1.5 & 1.8 & 1.7 \\
\hline & $\mathrm{Fe}$ & 0.5 & 1.1 & 1.0 \\
\hline & $\mathrm{Mn}$ & $<0.1$ & 0.1 & $<0.1$ \\
\hline & $\mathrm{Zn}$ & 0.5 & 0.4 & 0.5 \\
\hline & $\mathrm{Zr}$ & 0.4 & 1.1 & 0.9 \\
\hline & $\overline{\mathrm{U}}$ & 1.6 & 2.1 & 2.5 \\
\hline & $\mathrm{Pu}$ & 0.5 & 0.8 & 1.2 \\
\hline & $\mathrm{Am}$ & 24 & 50 & 86 \\
\hline Alkaline & $\mathrm{Ce}$ & 3.4 & 3.7 & 4.7 \\
\hline \multirow[t]{12}{*}{ Supernate } & Cs & 0.7 & 0.4 & 0.6 \\
\hline & $\mathrm{Sr}$ & 4.1 & 2.8 & 2.6 \\
\hline & Tc & 1.4 & 1.5 & 1.9 \\
\hline & $\mathrm{Y}$ & 5.1 & 5.1 & 5.2 \\
\hline & $\mathrm{Cr}$ & 0.5 & 0.6 & 0.8 \\
\hline & Co & 1.0 & 0.9 & 1.0 \\
\hline & $\mathrm{Fe}$ & 16 & 17 & 15 \\
\hline & $\mathrm{Mn}$ & 3.9 & 5.5 & 6.0 \\
\hline & $\mathrm{Zn}$ & 34 & 37 & 29 \\
\hline & $\mathrm{Zr}$ & 5.2 & 5.9 & 6.9 \\
\hline & $\mathrm{U}$ & $<0.1$ & 1.0 & 0.8 \\
\hline & Am & 1.3 & 3.2 & 3.9 \\
\hline
\end{tabular}


4. Phenolsulfonic-Formaldehyde (PSF) Composite Absorbers. These two composites were prepared and provided to us by Dr. Jerzy Narbutt, Head, Department of Radiochemistry, Institute of Nuclear Chemistry and Technology, Warsaw, Poland. In these two composite resins, the inorganic exchange material is dispersed in a phenolsulfonic-formaldehyde matrix..$^{14}$ These composites were used as received. a. TiFC-PSF Titanium Hexacyanoferrate Composite. The titanium hexacyanoferrate content of the TiFC-PSF composite was approximately $17 \%$.

TiFC-PSF sorbs cesium weakly from acid-dissolved sludge solution and more strongly from acidified supernate solution, although the sorption kinetics appear slow. It sorbs many elements well from alkaline supernate solution.

Table 53. TiFC-PSF Composite: Distribution of 14 Elements from Three Simulant Solutions for Hanford Tank 102-SY

\begin{tabular}{|c|c|c|c|c|}
\hline \multirow[b]{2}{*}{ Solution } & \multirow[b]{2}{*}{ Element } & \multicolumn{3}{|c|}{ Kd Value for Specified Time } \\
\hline & & $30 \mathrm{~min}$ & $2 \mathrm{~h}$ & $6 \mathrm{~h}$ \\
\hline Acid-Dissolved & $\mathrm{Ce}$ & $<0.1$ & 0.3 & 0.2 \\
\hline \multirow[t]{13}{*}{ Sludge } & Cs & 1.8 & 2.7 & 4.7 \\
\hline & $\mathrm{Sr}$ & 0.3 & 0.8 & 0.9 \\
\hline & $\mathrm{Tc}$ & 0.3 & 0.4 & 0.6 \\
\hline & $Y$ & 0.2 & 0.3 & 0.4 \\
\hline & $\mathrm{Cr}$ & 0.7 & 0.9 & 0.9 \\
\hline & $\mathrm{Co}$ & 0.6 & 0.7 & 0.8 \\
\hline & $\mathrm{Fe}$ & 0.4 & 0.3 & 0.3 \\
\hline & Mn & 0.4 & 0.3 & 0.5 \\
\hline & Zn & 0.4 & 0.2 & 0.5 \\
\hline & $\mathrm{Zr}$ & 0.8 & 0.8 & 1.7 \\
\hline & $\mathrm{U}$ & $<0.1$ & 0.2 & 0.2 \\
\hline & $\mathrm{Pu}$ & 0.4 & 0.1 & 0.3 \\
\hline & Am & 0.2 & 0.2 & 0.1 \\
\hline Acidified & $\mathrm{Ce}$ & 2.1 & 4.7 & 7.4 \\
\hline \multirow[t]{13}{*}{ Supernate } & Cs & 17 & 80 & 255 \\
\hline & $\mathrm{Sr}$ & 1.5 & 2.3 & 2.3 \\
\hline & $\mathrm{Tc}$ & 0.6 & 0.7 & 0.6 \\
\hline & $\mathrm{Y}$ & 2.0 & 4.3 & 5.8 \\
\hline & $\mathrm{Cr}$ & 0.1 & 0.9 & 1.5 \\
\hline & $\mathrm{Co}$ & 1.9 & 2.2 & 3.0 \\
\hline & $\mathrm{Fe}$ & $<0.1$ & $<0.1$ & $<0.1$ \\
\hline & $\mathrm{Mn}$ & 1.2 & 2.3 & 2.0 \\
\hline & $\mathrm{Zn}$ & 1.5 & 2.6 & 3.6 \\
\hline & $\mathrm{Zr}$ & $<0.1$ & $<0.1$ & $<0.1$ \\
\hline & $\mathrm{U}$ & 1.2 & 2.7 & 5.6 \\
\hline & $\mathrm{Pu}$ & 0.4 & 0.5 & 0.4 \\
\hline & $\mathrm{Am}$ & 1.7 & 3.6 & 5.3 \\
\hline Alkaline & $\mathrm{Ce}$ & 4.3 & 16 & 40 \\
\hline \multirow[t]{12}{*}{ Supernate } & Cs & 59 & 102 & 102 \\
\hline & $\mathrm{Sr}$ & 144 & 260 & 254 \\
\hline & $\mathrm{Tc}$ & $<0.1$ & $<0.1$ & $<0.1$ \\
\hline & $Y$ & 9.7 & 25 & 37 \\
\hline & $\mathrm{Cr}$ & $<0.1$ & 0.3 & 0.3 \\
\hline & $\mathrm{Co}$ & 0.6 & 1.7 & 2.4 \\
\hline & $\mathrm{Fe}$ & 62 & 159 & 257 \\
\hline & Mn & 9.9 & 33 & 87 \\
\hline & $\mathrm{Zn}$ & 30 & 211 & 335 \\
\hline & $\mathrm{Zr}$ & 73 & 192 & 222 \\
\hline & $\mathrm{U}$ & 25 & 93 & 135 \\
\hline & $\mathrm{Am}$ & 4.9 & 21 & 53 \\
\hline
\end{tabular}


b. CoFC-PSF Cobalt Hexacyanoferrate Composite. The cobalt hexacyanoferrate content of the CoFC-PSF composite was approximately $20 \%$.

CoFC-PSF sorbs only cesium at a useful level from acid-dissolved sludge solution. It sorbs cesium strongly and quite selectively from acidified supernate solution. It strongly sorbs cesium and strontium from alkaline supernate solution, although several other elements also sorb well from this solution.

Table 54. CoFC-PSF Composite: Distribution of 14 Elements from Three Simulant Solutions for Hanford Tank 102-SY

\begin{tabular}{|c|c|c|c|c|}
\hline \multirow[b]{2}{*}{ Solution } & \multirow[b]{2}{*}{ Element } & \multicolumn{3}{|c|}{ Kd Value for Specified Time } \\
\hline & & $30 \mathrm{~min}$ & $2 \mathrm{~h}$ & $6 \mathrm{~h}$ \\
\hline \multirow{14}{*}{$\begin{array}{l}\text { Acid-Dissolved } \\
\text { Sludge }\end{array}$} & $\mathrm{Ce}$ & 0.6 & 1.2 & 1.0 \\
\hline & $\mathrm{Cs}$ & 1.9 & 5.8 & 10 \\
\hline & $\mathrm{Sr}$ & 0.9 & 1.7 & 1.5 \\
\hline & $\mathrm{Tc}$ & 0.6 & 1.4 & 1.3 \\
\hline & $\mathrm{Y}$ & 0.8 & 1.2 & 1.2 \\
\hline & $\mathrm{Cr}$ & 0.8 & 2.0 & 1.4 \\
\hline & Co & 0.8 & 1.5 & 1.3 \\
\hline & $\mathrm{Fe}$ & 0.1 & 0.1 & $<0.1$ \\
\hline & $\mathrm{Mn}$ & $<0.1$ & 0.3 & 0.5 \\
\hline & $\mathrm{Zn}$ & 0.2 & 0.3 & 0.5 \\
\hline & $\mathrm{Zr}$ & 1.1 & 1.6 & 1.9 \\
\hline & $U$ & 0.4 & 0.1 & 0.6 \\
\hline & $\mathrm{Pu}$ & 0.2 & 0.3 & 0.6 \\
\hline & $\mathrm{Am}$ & $<0.1$ & $<0.1$ & 0.1 \\
\hline \multirow{14}{*}{$\begin{array}{l}\text { Acidified } \\
\text { Supernate }\end{array}$} & $\mathrm{Ce}$ & 1.3 & 2.8 & 3.9 \\
\hline & Cs & 39 & 263 & 880 \\
\hline & $\mathrm{Sr}$ & 0.5 & 1.2 & 1.4 \\
\hline & Tc & 0.6 & 0.4 & 0.6 \\
\hline & Y & 1.7 & 3.5 & 4.0 \\
\hline & $\mathrm{Cr}$ & $<0.1$ & 0.5 & 0.7 \\
\hline & Co & 1.8 & 3.4 & 5.7 \\
\hline & $\mathrm{Fe}$ & $<0.1$ & $<0.1$ & $<0.1$ \\
\hline & $\mathrm{Mn}$ & 0.9 & 1.3 & 1.4 \\
\hline & $\mathrm{Zn}$ & 1.5 & 4.1 & 8.9 \\
\hline & $\mathrm{Zr}$ & 0.2 & $<0.1$ & $<0.1$ \\
\hline & $\mathrm{U}$ & 1.2 & 2.6 & 4.0 \\
\hline & $\mathrm{Pu}$ & 0.2 & 0.1 & 0.3 \\
\hline & $\mathrm{Am}$ & 1.6 & 2.3 & 3.0 \\
\hline \multirow{13}{*}{$\begin{array}{l}\text { Alkaline } \\
\text { Supernate }\end{array}$} & $\mathrm{Ce}$ & 0.9 & 2.0 & 2.8 \\
\hline & Cs & 1154 & 3793 & 3575 \\
\hline & $\mathrm{Sr}$ & 84 & 293 & 1270 \\
\hline & $\mathrm{Tc}$ & $<0.1$ & $<0.1$ & $<0.1$ \\
\hline & $\mathrm{Y}$ & 3.7 & 11 & 23 \\
\hline & $\mathrm{Cr}$ & 0.3 & 0.2 & 0.3 \\
\hline & Co & 1.5 & 2.8 & 4.0 \\
\hline & $\mathrm{Fe}$ & 30 & 82 & 110 \\
\hline & $\mathrm{Mn}$ & 2.3 & 5.0 & 6.5 \\
\hline & $\mathrm{Zn}$ & 11 & 85 & 471 \\
\hline & $\mathrm{Zr}$ & 13 & 46 & 129 \\
\hline & $\mathrm{U}$ & 7.6 & 36 & 92 \\
\hline & Am & $<0.1$ & 0.8 & 1.1 \\
\hline
\end{tabular}


5. Los Alamos-Prepared Composites. We prepared metal phosphate composites by loading the selected metal ion on Duolite ${ }^{\mathrm{TM}} \mathrm{C}-467$ phosphonate cation exchange resin, obtained from Rohm \& Haas Co., Philadelphia, PA, and then heating the loaded resin stepwise in a tube furnace (in a stream of nitrogen) to a final temperature of $600^{\circ} \mathrm{C}$. We first tested this technique by heating $1.002 \mathrm{~g}$ of unloaded, airdried Duolite ${ }^{\mathrm{TM}}$ resin for $2 \mathrm{~h}$ at $400^{\circ} \mathrm{C}$. We found that the resin weight had decreased to $589 \mathrm{mg}$. After the same portion of resin was heated for an additional hour at $500^{\circ} \mathrm{C}$ and one more hour at $850^{\circ} \mathrm{C}$, only $453 \mathrm{mg}$ remained.

We prepared metal oxide composites by impregnating Ambersorb ${ }^{\mathrm{TM}} 563$ porous carbon beads with the appropriate metal alkoxide, after which we hydrolyzed the alkoxide to the metal oxide within the pores of the support beads, using the procedure of Suzuki. ${ }^{15}$ 
a. LANL-ZrP Zirconium Phosphate. We dissolved an $8.75-\mathrm{g}$ portion of solid $\mathrm{ZrCl}_{4}$ (with quite a vigorous reaction) in $50 \mathrm{~mL}$ of $1.5 \mathrm{M} \mathrm{HCl}$ and added this solution to $50 \mathrm{~mL}$ of wet Duolite ${ }^{\mathrm{TM}} \mathrm{C}-467$ resin. We agitated this mixture on a 48rpm tube rotator for $2 \mathrm{~h}$ and then transferred the zirconiumloaded resin to a column, washed it with an excess of $0.5 \mathrm{M}$ $\mathrm{HCl}$, rinsed it well with water, and air-dried it.

After stepwise heating to $600^{\circ} \mathrm{C}$, the weight of an initial $3.126 \mathrm{~g}$ of zirconium-loaded, air-dried resin decreased to $1.177 \mathrm{~g}(37.7 \%)$. Stepwise heating of a sepa- rate $7.714-\mathrm{g}$ portion resulted in a weight of $3.772 \mathrm{~g}$ $(48.9 \%)$. The difference in final weight indicates that larger portions are less efficiently decomposed to the metal phosphates. The two portions were combined for use in this study.

LANL-Zrp sorbs none of the 14 elements at useful levels from acid-dissolved sludge or acidified supernate solution, whereas it sorbs many elements well from alkaline supernate solution.

\begin{tabular}{|c|c|c|c|c|}
\hline \multirow[b]{2}{*}{ Solution } & \multirow[b]{2}{*}{ Element } & \multicolumn{3}{|c|}{ Kd Value for Specified Time } \\
\hline & & $30 \mathrm{~min}$ & $2 \mathbf{h}$ & $6 \mathrm{~h}$ \\
\hline $\begin{array}{l}\text { Acid-Dissolved } \\
\text { Sludge }\end{array}$ & $\begin{array}{l}\mathrm{Ce} \\
\mathrm{Cs} \\
\mathrm{Sr} \\
\mathrm{Tc} \\
\mathrm{Y} \\
\mathrm{Cr} \\
\mathrm{Co} \\
\mathrm{Fe} \\
\mathrm{Mn} \\
\mathrm{Zn} \\
\mathrm{Zr} \\
\mathrm{U} \\
\mathrm{Pu} \\
\mathrm{Am}\end{array}$ & $\begin{array}{r}0.9 \\
1.0 \\
1.0 \\
1.0 \\
1.1 \\
1.4 \\
1.3 \\
0.3 \\
<0.1 \\
0.2 \\
2.0 \\
0.2 \\
0.5 \\
<0.1\end{array}$ & $\begin{array}{r}0.4 \\
1.1 \\
1.0 \\
1.0 \\
0.3 \\
1.1 \\
1.3 \\
0.1 \\
<0.1 \\
0.3 \\
2.4 \\
0.1 \\
0.3 \\
0.2\end{array}$ & $\begin{array}{l}0.6 \\
1.4 \\
1.2 \\
1.2 \\
0.8 \\
1.3 \\
1.1 \\
0.3 \\
0.2 \\
0.2 \\
3.3 \\
0.5 \\
0.6 \\
0.1\end{array}$ \\
\hline $\begin{array}{l}\text { Acidified } \\
\text { Supernate }\end{array}$ & $\begin{array}{l}\mathrm{Ce} \\
\mathrm{Cs} \\
\mathrm{Sr} \\
\mathrm{Tc} \\
\mathrm{Y} \\
\mathrm{Cr} \\
\mathrm{Co} \\
\mathrm{Fe} \\
\mathrm{Mn} \\
\mathrm{Zn} \\
\mathrm{Zr} \\
\mathrm{U} \\
\mathrm{Pu} \\
\mathrm{Am}\end{array}$ & $\begin{array}{r}0.2 \\
1.3 \\
<0.1 \\
0.7 \\
1.0 \\
0.3 \\
0.7 \\
<0.1 \\
0.3 \\
0.4 \\
0.7 \\
1.4 \\
<0.1 \\
0.3\end{array}$ & $\begin{array}{r}0.8 \\
2.2 \\
0.2 \\
1.0 \\
1.2 \\
0.3 \\
0.6 \\
<0.1 \\
0.6 \\
0.7 \\
0.2 \\
2.5 \\
0.1 \\
0.5\end{array}$ & $\begin{array}{r}0.8 \\
2.6 \\
0.1 \\
0.6 \\
1.0 \\
0.4 \\
0.4 \\
<0.1 \\
0.5 \\
0.9 \\
0.2 \\
2.6 \\
0.3 \\
0.4\end{array}$ \\
\hline
\end{tabular}


Table 55. LANL-ZrP Composite: Distribution of 14 Elements from Three Simulant Solutions for Hanford Tank 102-SY (Cont.)

\begin{tabular}{lccrr}
\hline & & \multicolumn{3}{c}{ Kd Value for Specified Time } \\
\cline { 2 - 5 } Solution & Element & $\mathbf{3 0} \mathbf{~}$ in & $\mathbf{2 ~ h}$ & $\mathbf{6 ~ h}$ \\
\hline Alkaline & $\mathrm{Ce}$ & 6.4 & 37 & 114 \\
Supernate & $\mathrm{Cs}$ & 0.9 & 1.3 & 1.4 \\
& $\mathrm{Sr}$ & 34 & 108 & 343 \\
& $\mathrm{Tc}$ & $<0.1$ & $<0.1$ & $<0.1$ \\
& $\mathrm{Y}$ & 6.7 & 20 & 39 \\
& $\mathrm{Cr}$ & 0.1 & 0.4 & 0.3 \\
& $\mathrm{Co}$ & 2.6 & 3.9 & 4.9 \\
& $\mathrm{Fe}$ & 26 & 162 & 195 \\
$\mathrm{Mn}$ & 12 & 106 & 96 \\
& $\mathrm{Zn}$ & 8.8 & 30 & 104 \\
& $\mathrm{Zr}$ & 18 & 52 & 159 \\
& $\mathrm{U}$ & 5.3 & 14 & 20 \\
& $\mathrm{Am}$ & 9.4 & 102 & 75 \\
\hline \hline
\end{tabular}


b. LANL-TiP Titanium Phosphate. We mixed a 7.10-g portion of liquid $\mathrm{TiCl}_{4}$ (with quite a vigorous reaction) in $50 \mathrm{~mL}$ of $1.5 \mathrm{M} \mathrm{HCl}$ and added the resulting solution to $50 \mathrm{~mL}$ of wet Duolite ${ }^{\mathrm{TM}} \mathrm{C}-467$ resin. We agitated this mixture on a 48-rpm tube rotator for $2 \mathrm{~h}$ and then transferred the titanium-loaded resin to a column, washed it with an excess of $0.5 \mathrm{M} \mathrm{HCl}$, rinsed it well with water, and air-dried it.

After stepwise heating to $600^{\circ} \mathrm{C}$, the weight of an initial 5.610-g portion of this titanium-loaded, air-dried resin decreased to $2.409 \mathrm{~g}(42.9 \%)$. Stepwise heating of a separate $5.669-\mathrm{g}$ portion resulted in a weight of $2.443 \mathrm{~g}$ $(43.1 \%)$. The difference between the two final weights indicates that when the weights of the initial portions are reasonably constant, the decomposition to metal phosphates is fairly reproducible. The two portions were combined for use in this study.

LANL-TiP sorbs none of the 14 elements at useful levels from acid-dissolved sludge or acidified supernate solution. However, it sorbs nearly half of the elements studied at useful levels from alkaline supernate solution.

Table 56. LANL-TiP Composite: Distribution of 14 Elements from Three Simulant Solutions for Hanford Tank 102-SY

\begin{tabular}{lcccc}
\hline & & \multicolumn{3}{c}{ Kd Value for Specified Time } \\
\cline { 2 - 5 } Solution & Element & $\mathbf{3 0 ~} \mathbf{~ m i n}$ & $\mathbf{2 ~ h}$ & $\mathbf{6 ~ h}$ \\
\hline Acid-Dissolved & $\mathrm{Ce}$ & 0.6 & 0.3 & 0.3 \\
Sludge & $\mathrm{Cs}$ & 2.0 & 2.4 & 4.0 \\
& $\mathrm{Sr}$ & 1.0 & 0.4 & 0.7 \\
& $\mathrm{Tc}$ & 1.6 & 0.7 & 0.9 \\
$\mathrm{Y}$ & 1.0 & 0.5 & 0.4 \\
& $\mathrm{Cr}$ & 1.2 & 1.1 & 0.8 \\
& $\mathrm{Co}$ & $\mathbf{0 . 7}$ & 0.2 & 0.5 \\
& $\mathrm{Fe}$ & 0.1 & 0.1 & 0.1 \\
$\mathrm{Mn}$ & $<0.1$ & $<0.1$ & 0.1 \\
& $\mathrm{Zn}$ & 0.2 & 0.2 & 0.2 \\
& $\mathrm{Zr}$ & 1.8 & 1.7 & 2.1 \\
& $\mathrm{U}$ & 0.4 & 0.3 & 0.3 \\
& $\mathrm{Pu}$ & 0.6 & 0.7 & 0.8 \\
& $\mathrm{Am}$ & $<0.1$ & $<0.1$ & $<0.1$ \\
\hline Acidified & $\mathrm{Ce}$ & 1.0 & 0.8 & 0.9 \\
Supernate & $\mathrm{Cs}$ & 2.1 & 2.6 & 3.5 \\
& $\mathrm{Sr}$ & 0.4 & 0.1 & 0.1 \\
& $\mathrm{Tc}$ & 1.1 & 0.7 & 0.7 \\
& $\mathrm{Y}$ & 1.2 & 0.9 & 0.8 \\
& $\mathrm{Cr}$ & 0.3 & 0.5 & 0.3 \\
& $\mathrm{Co}$ & 1.0 & 0.5 & 0.4 \\
& $\mathrm{Fe}$ & $<0.1$ & 0.3 & 0.2 \\
& $\mathrm{Mn}$ & 0.3 & 0.1 & 0.5 \\
& $\mathrm{Zn}$ & 1.1 & 1.7 & 2.6 \\
& $\mathrm{Zr}$ & $<0.1$ & 0.1 & 0.1 \\
& $\mathrm{U}$ & 2.5 & 3.6 & 3.8 \\
& $\mathrm{Pu}$ & $<0.1$ & 0.5 & 0.2 \\
& $\mathrm{Am}$ & 1.1 & 1.0 & 0.9 \\
\hline & & & & \\
& & &
\end{tabular}


Table 56. LANL-TiP Composite: Distribution of 14 Elements from Three Simulant Solutions for Hanford Tank 102-SY (Cont.)

\begin{tabular}{|c|c|c|c|c|}
\hline \multirow[b]{2}{*}{ Solution } & \multirow[b]{2}{*}{ Element } & \multicolumn{3}{|c|}{ Kd Value for Specified Time } \\
\hline & & $30 \mathrm{~min}$ & $2 \mathrm{~h}$ & $6 \mathrm{~h}$ \\
\hline Alkaline & $\mathrm{Ce}$ & 30 & 61 & 22 \\
\hline \multirow[t]{12}{*}{ Supernate ${ }^{a, b}$} & Cs & 0.7 & 1.1 & 1.4 \\
\hline & $\mathrm{Sr}$ & 76 & 117 & 16 \\
\hline & Tc & $<0.1$ & $<0.1$ & $<0.1$ \\
\hline & $\mathrm{Y}$ & 20 & 31 & 20 \\
\hline & $\mathrm{Cr}$ & $<0.1$ & 0.2 & 0.3 \\
\hline & Co & 2.0 & 4.0 & 6.1 \\
\hline & $\mathrm{Fe}$ & 75 & 53 & $>1000$ \\
\hline & $\mathrm{Mn}$ & 47 & 49 & $>1000$ \\
\hline & $\mathrm{Zn}$ & 173 & 118 & $>1000$ \\
\hline & $\mathrm{Zr}$ & 88 & 106 & 34 \\
\hline & $\mathrm{U}$ & 80 & 51 & 79 \\
\hline & Am & 40 & 44 & 908 \\
\hline
\end{tabular}

\footnotetext{
${ }^{a}$ After the absorber/solution contact period of $6 \mathrm{~h}$, we passed the alkaline supernate solutions containing cesium, strontium, iron, manganese, zinc, uranium, and americium through a $0.45-\mu \mathrm{m}$ filter to remove visible particles.

${ }^{b}$ The Kd value for sorbing strontium from alkaline supernate solution after $6 \mathrm{~h}$ is obviously in error and should be disregarded.
} 
c. LANL-TiO Titanium Dioxide. We diluted a 30-mL portion of titanium butoxide with $60 \mathrm{~mL}$ of cyclohexane and added $79.1 \mathrm{~g}$ of Ambersorb ${ }^{\mathrm{TM}} 563$ porous carbon beads, obtained from Rohm \& Haas, Philadelphia, PA, to absorb the liquid. After the volatile solvent evaporated, we heated the beads in $0.1 \mathrm{M}$ nitric acid for $2 \mathrm{~h}$. The loaded beads were washed with an excess of water and air-dried to yield $94.3 \mathrm{~g}$ ( $119.3 \%$ of the added $\mathrm{Ambersorb}^{\mathrm{TM}}$ beads). If the $\mathrm{TiO}_{2}$ were anhydrous, which it is not, this would represent 2.4 mmoles of titanium per initial gram of Ambersorb ${ }^{\mathrm{TM}}$ beads.

LANL-TiO sorbs technetium and zirconium from acid-dissolved sludge solution, although the sorption kinetics are slow. It sorbs technetium well and uranium moderately from acidified supernate solution. It sorbs strontium, zirconium, zinc, and iron well and several other elements moderately from alkaline supernate solution.

Table 57. LANL-TiO Composite: Distribution of 14 Elements from Three Simulant Solutions for Hanford Tank 102-SY

\begin{tabular}{|c|c|c|c|c|}
\hline \multirow[b]{2}{*}{ Solution } & \multirow[b]{2}{*}{ Element } & \multicolumn{3}{|c|}{ Kd Value for Specified Time } \\
\hline & & $30 \mathrm{~min}$ & $2 \mathrm{~h}$ & $6 \mathrm{~h}$ \\
\hline Acid-Dissolved & $\mathrm{Ce}$ & 0.8 & 0.8 & 0.6 \\
\hline \multirow[t]{13}{*}{ Sludge. } & Cs & 0.8 & 0.4 & 0.3 \\
\hline & $\mathrm{Sr}$ & 0.3 & 0.1 & 0.1 \\
\hline & $\mathrm{Tc}$ & 19 & 40 & 57 \\
\hline & $\mathbf{Y}$ & 0.8 & 0.8 & 0.6 \\
\hline & $\mathrm{Cr}$ & 0.5 & 0.6 & 0.5 \\
\hline & Co & 0.6 & 0.7 & 0.6 \\
\hline & $\mathrm{Fe}$ & $<0.1$ & 0.1 & 0.1 \\
\hline & $\mathrm{Mn}$ & 0.3 & 0.1 & 0.1 \\
\hline & $\mathrm{Zn}$ & 0.4 & 0.2 & 0.2 \\
\hline & $\mathrm{Zr}$ & 4.4 & 10 & 28 \\
\hline & $\mathrm{U}$ & 0.3 & 0.2 & 0.4 \\
\hline & $\mathrm{Pu}$ & 1.1 & 1.6 & 2.1 \\
\hline & $\mathrm{Am}$ & 0.2 & 0.2 & 0.2 \\
\hline Acidified & $\mathrm{Ce}$ & 1.7 & 2.2 & 1.6 \\
\hline \multirow{13}{*}{ Supernate } & Cs & 0.1 & $<0.1$ & $<0.1$ \\
\hline & $\mathrm{Sr}$ & $<0.1$ & 0.1 & 0.1 \\
\hline & $\mathrm{Tc}$ & 54 & 185 & 290 \\
\hline & $\mathrm{Y}$ & 0.7 & 0.8 & 0.5 \\
\hline & $\mathrm{Cr}$ & 0.4 & 1.1 & 1.7 \\
\hline & Co & 0.4 & 0.7 & 0.4 \\
\hline & $\mathrm{Fe}$ & $<0.1$ & 0.5 & 1.0 \\
\hline & $\mathrm{Mn}$ & 0.4 & 0.1 & 0.1 \\
\hline & $\mathrm{Zn}$ & $<0.1$ & $<0.1$ & 0.2 \\
\hline & $\mathrm{Zr}$ & 0.4 & 0.8 & 1.0 \\
\hline & $\mathrm{U}$ & 3.8 & 9.1 & 19 \\
\hline & $\mathbf{P u}$ & 0.3 & 0.5 & 1.1 \\
\hline & $\mathrm{Am}$ & 1.3 & 1.5 & 1.5 \\
\hline Alkaline & $\mathrm{Ce}$ & 2.3 & 3.5 & 6.7 \\
\hline \multirow{12}{*}{ Supernate } & $\mathrm{Cs}$ & 0.4 & 0.5 & 0.4 \\
\hline & $\mathrm{Sr}$ & 51 & 129 & 660 \\
\hline & $\mathrm{Tc}$ & 2.2 & 1.8 & 1.8 \\
\hline & $\mathrm{Y}$ & 6.9 & 18 & 56 \\
\hline & $\mathrm{Cr}$ & $<0.1$ & 0.2 & 0.1 \\
\hline & Co & 6.4 & 12 & 18 \\
\hline & $\mathrm{Fe}$ & 41 & 83 & 128 \\
\hline & $\mathrm{Mn}$ & 4.3 & 7.3 & 13 \\
\hline & $\mathrm{Zn}$ & 51 & 215 & 448 \\
\hline & $\mathrm{Zr}$ & 35 & 205 & 570 \\
\hline & $\mathbf{U}$ & 14 & 41 & 45 \\
\hline & $\mathrm{Am}$ & $<0.1$ & 0.7 & 5.0 \\
\hline
\end{tabular}


d. LANL-ZrO Zirconium Dioxide. We diluted a $30-\mathrm{mL}$ portion of zirconium butoxide-butanol with $60 \mathrm{~mL}$ of cyclohexane and added $80.4 \mathrm{~g}$ of Ambersorb ${ }^{\mathrm{TM}} 563$ porous carbon beads, obtained from Rohm \& Haas, Philadelphia, PA, to absorb the liquid. After the volatile solvent evaporated, we heated the beads in $0.1 \mathrm{M}$ nitric acid for $2 \mathrm{~h}$. We then washed the loaded beads with an excess of water and airdried them to yield $91.33 \mathrm{~g}$ ( $123.2 \%$ of the added Ambersorb ${ }^{\mathrm{TM}}$ beads). If the $\mathrm{ZrO}_{2}$ were anhydrous, which it is not, this would represent $1.1 \mathrm{mmoles}$ of zirconium per initial gram of Ambersorb $^{\mathrm{TM}}$ beads.

LANL-ZrO sorbs technetium from acid-dissolved sludge solution, although with slow sorption kinetics. It sorbs technetium strongly and selectively from acidified supernate solution, again with slow sorption kinetics. For sorption from alkaline supernate solution, zirconium and strontium offer triple-digit Kd values, whereas several other elements sorb at lower but still useful levels.

Table 58. LANL-ZrO Composite: Distribution of 14 Elements from Three Simulant Solutions for Hanford Tank 102-SY

\begin{tabular}{|c|c|c|c|c|}
\hline \multirow[b]{2}{*}{ Solution } & \multirow[b]{2}{*}{ Element } & \multicolumn{3}{|c|}{ Kd Value for Specified Time } \\
\hline & & $30 \mathrm{~min}$ & $2 \mathrm{~h}$ & $6 \mathrm{~h}$ \\
\hline \multirow{14}{*}{$\begin{array}{l}\text { Acid-Dissolved } \\
\text { Sludge }\end{array}$} & $\mathrm{Ce}$ & 0.7 & 0.8 & 0.8 \\
\hline & Cs & 0.5 & 0.3 & 0.5 \\
\hline & $\mathrm{Sr}$ & 0.9 & 0.2 & 0.3 \\
\hline & Tc & 17 & 41 & 60 \\
\hline & $\mathrm{Y}$ & 0.8 & 1.0 & 1.0 \\
\hline & $\mathrm{Cr}$ & 1.0 & 0.7 & 0.6 \\
\hline & $\mathrm{Co}$ & 0.8 & 0.5 & 0.5 \\
\hline & $\mathrm{Fe}$ & 0.6 & 0.2 & 0.2 \\
\hline & $\mathrm{Mn}$ & $<0.1$ & 0.2 & 0.1 \\
\hline & $\mathrm{Zn}$ & 0.2 & 0.2 & 0.2 \\
\hline & $\mathrm{Zr}$ & 1.7 & 2.8 & 3.7 \\
\hline & $\mathrm{U}$ & 0.1 & $<0.1$ & 0.3 \\
\hline & $\mathbf{P u}$ & 0.6 & 0.7 & 0.8 \\
\hline & Am & 0.4 & 0.2 & 0.3 \\
\hline \multirow{14}{*}{$\begin{array}{l}\text { Acidified } \\
\text { Supernate }\end{array}$} & $\mathrm{Ce}$ & $<0.1$ & 0.2 & 0.6 \\
\hline & Cs & $<0.1$ & $<0.1$ & 0.1 \\
\hline & $\mathrm{Sr}$ & 1.0 & 0.6 & 0.6 \\
\hline & Tc & 35 & 148 & 258 \\
\hline & $Y$ & 0.1 & $<0.1$ & $<0.1$ \\
\hline & $\mathrm{Cr}$ & 0.4 & 0.6 & 1.2 \\
\hline & Co & 0.4 & 0.6 & 0.5 \\
\hline & $\mathrm{Fe}$ & $<0.1$ & 0.6 & 0.4 \\
\hline & $\mathrm{Mn}$ & 0.1 & 0.7 & 0.5 \\
\hline & $\mathrm{Zn}$ & 0.3 & 0.3 & 0.3 \\
\hline & $\mathrm{Zr}$ & $<0.1$ & 0.7 & 0.9 \\
\hline & $\mathrm{U}$ & 1.4 & 2.2 & 2.9 \\
\hline & $\mathrm{Pu}$ & 0.1 & 0.4 & 0.6 \\
\hline & Am & $<0.1$ & $<0.1$ & $<0.1$ \\
\hline \multirow{13}{*}{$\begin{array}{l}\text { Alkaline } \\
\text { Supernate }\end{array}$} & $\mathrm{Ce}$ & 1.6 & 2.8 & 4.9 \\
\hline & Cs & $<0.1$ & $<0.1$ & $<0.1$ \\
\hline & $\mathrm{Sr}$ & 60 & 164 & 526 \\
\hline & $\mathrm{Tc}$ & 0.4 & 0.6 & 0.6 \\
\hline & $\mathrm{Y}$ & 6.7 & 17 & 41 \\
\hline & $\mathrm{Cr}$ & $<0.1$ & $<0.1$ & $<0.1$ \\
\hline & Co & 4.3 & 7.6 & 10 \\
\hline & $\mathrm{Fe}$ & 35 & 71 & 92 \\
\hline & $\mathrm{Mn}$ & 3.4 & 5.3 & 7.2 \\
\hline & $\mathrm{Zn}$ & 19 & 37 & 53 \\
\hline & $\mathrm{Zr}$ & 58 & 273 & 611 \\
\hline & $\mathrm{U}$ & 9.7 & 17 & 27 \\
\hline & Am & $<0.1$ & $<0.1$ & 0.5 \\
\hline
\end{tabular}


e. LANL-NbO Niobium Pentoxide. We diluted a $50-\mathrm{mL}$ portion of niobium ethoxide with $100 \mathrm{~mL}$ of cyclohexane and added $113.9 \mathrm{~g}$ of Ambersorb ${ }^{\mathrm{TM}} 563$ porous carbon beads, obtained from Rohm \& Haas, Philadelphia, PA, to absorb the liquid. After the volatile solvent evaporated, we heated the beads in $0.1 \mathrm{M}$ nitric acid for $2 \mathrm{~h}$. We then washed the loaded beads with an excess of water and air-dried them to yield $140.30 \mathrm{~g}$ (123.2\% of the added Ambersorb ${ }^{\mathrm{TM}}$ beads). If the $\mathrm{Nb}_{2} \mathrm{O}_{5}$ were anhydrous, which it is not, this would represent 1.7 mmoles of niobium per initial gram of Ambersorb $^{\text {TM }}$ beads.

LANL-NbO sorbs technetium and zirconium moderately from acid-dissolved sludge solution. It sorbs technetium more strongly from acidified supernate solution, although with slow sorption kinetics. It sorbs strontium well and many other elements moderately from alkaline supernate solution.

Table 59. LANL-NbO Composite: Distribution of 14 Elements from Three Simulant Solutions for Hanford Tank 102-SY

\begin{tabular}{|c|c|c|c|c|}
\hline \multirow[b]{2}{*}{ Solution } & \multirow[b]{2}{*}{ Element } & \multicolumn{3}{|c|}{ Kd Value for Specified Time } \\
\hline & & $30 \mathrm{~min}$ & $2 \mathrm{~h}$ & $6 \mathrm{~h}$ \\
\hline Acid-Dissolved & $\mathrm{Ce}$ & 0.3 & 0.5 & 0.5 \\
\hline \multirow{13}{*}{ Sludge } & Cs & 0.5 & 0.9 & 0.7 \\
\hline & $\mathrm{Sr}$ & 0.1 & 0.3 & 0.1 \\
\hline & Tc & 6.9 & 17 & 31 \\
\hline & $\mathrm{Y}$ & 0.8 & 0.3 & 0.5 \\
\hline & $\mathrm{Cr}$ & 0.6 & 0.5 & 0.6 \\
\hline & Co & 0.3 & 0.4 & 0.6 \\
\hline & $\mathrm{Fe}$ & 0.5 & 0.3 & 0.4 \\
\hline & $\mathrm{Mn}$ & 0.1 & 0.2 & 0.1 \\
\hline & $\mathrm{Zn}$ & 0.3 & 0.3 & 0.3 \\
\hline & $\mathrm{Zr}$ & 2.2 & 6.3 & 14 \\
\hline & $\mathrm{U}$ & $<0.1$ & 0.6 & 0.5 \\
\hline & $\mathrm{Pu}$ & 1.5 & 2.4 & 3.4 \\
\hline & Am & 0.2 & 0.1 & 0.1 \\
\hline Acidified & $\mathrm{Ce}$ & 1.7 & 2.4 & 2.8 \\
\hline \multirow[t]{13}{*}{ Supernate } & Cs & 4.5 & 5.6 & 6.8 \\
\hline & $\mathrm{Sr}$ & 0.5 & 0.1 & 0.2 \\
\hline & $\mathrm{Tc}$ & 28 & 134 & 271 \\
\hline & $\mathrm{Y}$ & 0.9 & 0.8 & 0.8 \\
\hline & $\mathrm{Cr}$ & 0.9 & 1.6 & 2.9 \\
\hline & Co & 0.4 & 0.6 & 0.6 \\
\hline & $\mathrm{Fe}$ & $<0.1$ & $<0.1$ & 0.2 \\
\hline & $\mathrm{Mn}$ & 0.8 & 0.7 & 0.3 \\
\hline & $\mathrm{Zn}$ & 1.3 & 0.8 & 0.7 \\
\hline & $\mathrm{Zr}$ & $<0.1$ & 0.2 & 0.6 \\
\hline & $\mathrm{U}$ & 2.3 & 4.1 & 7.3 \\
\hline & $\mathrm{Pu}$ & 0.5 & 0.2 & 0.2 \\
\hline & Am & 1.1 & 1.7 & 2.9 \\
\hline Alkaline & $\mathrm{Ce}$ & 3.1 & 5.0 & 6.8 \\
\hline \multirow[t]{12}{*}{ Supernate } & Cs & 0.6 & 0.7 & 1.0 \\
\hline & $\mathrm{Sr}$ & 31 & 63 & 129 \\
\hline & Tc & 2.8 & 2.8 & 3.0 \\
\hline & $\mathrm{Y}$ & 6.4 & 10 & 15 \\
\hline & $\mathrm{Cr}$ & $<0.1$ & $<0.1$ & 0.2 \\
\hline & $\mathrm{Co}$ & 5.7 & 15 & 22 \\
\hline & $\mathrm{Fe}$ & 6.9 & 22 & 34 \\
\hline & Mn & $<0.1$ & 5.4 & 12 \\
\hline & $\mathrm{Zn}$ & 5.5 & 12 & 15 \\
\hline & $\mathrm{Zr}$ & 12 & 12 & 14 \\
\hline & $\mathrm{U}$ & 7.2 & 11 & 7.5 \\
\hline & $\mathrm{Am}$ & $<0.1$ & 0.2 & 4.7 \\
\hline
\end{tabular}


6. Liquid Extractants. We prepared and evaluated porous beads impregnated with 12 different liquid extractants.

a. $L I X^{\mathrm{TM}}-1010$. We diluted $10 \mathrm{~mL}$ of LIXTM-1010 (di-2-ethylhexylphosphoric acid), obtained from Henkel Corporation, Tucson, AZ, with $20 \mathrm{~mL}$ of cyclohexane and added $25.76 \mathrm{~g}$ of AmbersorbTM 563 porous carbon beads, obtained from Rohm \& Haas Co., Philadelphia, PA, to absorb the liquid. The cyclohexane evaporated to yield $34.7 \mathrm{~g}$ of the loaded AmbersorbTM beads.
LIX ${ }^{\mathrm{TM}}-1010$ sorbs zirconium moderately from aciddissolved sludge solution, but with slow sorption kinetics. It sorbs americium and uranium at useful levels from acidified sludge solution. Because the $\mathrm{Kd}$ values for americium sorption are much higher than those for cerium or yttrium, a trivalent actinide/lanthanide group separation from this solution may be achievable. Yttrium and strontium are sorbed with double-digit $\mathrm{Kd}$ values from alkaline supernate solution.

Table 60. LIX ${ }^{\mathrm{TM}}$-1010: Distribution of 14 Elements from Three Simulant Solutions for Hanford Tank 102-SY

\begin{tabular}{|c|c|c|c|c|}
\hline \multirow{2}{*}{ Solution } & \multirow[b]{2}{*}{ Element } & \multicolumn{3}{|c|}{ Kd Value for Specified Time } \\
\hline & & $30 \mathrm{~min}$ & $2 \mathrm{~h}$ & $6 \mathrm{~h}$ \\
\hline \multirow{14}{*}{$\begin{array}{l}\text { Acid-Dissolved } \\
\text { Sludge }\end{array}$} & $\mathrm{Ce}$ & 0.8 & 0.9 & 0.6 \\
\hline & Cs & $<0.1$ & 0.3 & 0.1 \\
\hline & $\mathrm{Sr}$ & $<0.1$ & 0.2 & 0.1 \\
\hline & $\mathrm{Tc}$ & 1.8 & 2.7 & 4.0 \\
\hline & $\mathrm{Y}$ & 1.1 & 0.5 & 0.4 \\
\hline & $\mathrm{Cr}$ & 0.8 & 0.8 & 0.7 \\
\hline & Co & 0.4 & 0.1 & 0.2 \\
\hline & $\mathrm{Fe}$ & 0.4 & 0.3 & 0.5 \\
\hline & $\mathrm{Mn}$ & $<0.1$ & 0.2 & 0.1 \\
\hline & $\mathrm{Zn}$ & $<0.1$ & 0.4 & 0.2 \\
\hline & $\mathrm{Zr}$ & 3.6 & 10 & 25 \\
\hline & $\mathrm{U}$ & 0.1 & $<0.1$ & 0.5 \\
\hline & $\mathrm{Pu}$ & 0.8 & 0.7 & 0.9 \\
\hline & $\mathrm{Am}$ & 0.1 & 0.1 & 0.1 \\
\hline \multirow{14}{*}{$\begin{array}{l}\text { Aciditied } \\
\text { Supernate }\end{array}$} & $\mathrm{Ce}$ & 0.5 & 1.5 & 1.7 \\
\hline & $\mathrm{Cs}$ & $<0.1$ & $<0.1$ & 0.2 \\
\hline & $\mathrm{Sr}$ & 0.7 & 0.7 & 0.4 \\
\hline & Tc & 1.0 & 1.7 & 3.0 \\
\hline & $\mathrm{Y}$ & 1.0 & 1.2 & 1.3 \\
\hline & $\mathrm{Cr}$ & 0.3 & 0.6 & 0.6 \\
\hline & Co & 1.0 & 0.9 & 0.6 \\
\hline & $\mathrm{Fe}$ & $<0.1$ & 0.1 & $<0.1$ \\
\hline & $\mathrm{Mn}$ & 4.9 & 5.7 & 5.3 \\
\hline & $\mathrm{Zn}$ & 3.6 & 4.0 & 4.0 \\
\hline & $\mathrm{Zr}$ & 0.1 & 0.8 & 0.5 \\
\hline & $\mathrm{U}$ & 4.3 & 7.1 & 15 \\
\hline & $\mathrm{Pu}$ & 0.1 & $<0.1$ & 0.1 \\
\hline & $\mathrm{Am}$ & 10 & 27 & 64 \\
\hline Alkaline & $\mathrm{Ce}$ & 3.0 & 5.0 & 5.0 \\
\hline \multirow[t]{12}{*}{ Supernate } & Cs & $<0.1$ & $<0.1$ & $<0.1$ \\
\hline & $\mathrm{Sr}$ & 4.4 & 11 & 17 \\
\hline & $\mathrm{Tc}$ & 0.2 & 0.3 & 0.4 \\
\hline & $\mathrm{Y}$ & 4.4 & 13 & 33 \\
\hline & $\mathrm{Cr}$ & $<0.1$ & 0.2 & 0.3 \\
\hline & Co & $<0.1$ & 0.2 & 0.5 \\
\hline & $\mathrm{Fe}$ & 2.9 & 6.0 & 7.8 \\
\hline & $\mathrm{Mn}$ & 0.2 & 0.3 & 0.7 \\
\hline & $\mathrm{Zn}$ & $<0.1$ & 0.1 & 0.2 \\
\hline & $\mathrm{Zr}$ & 0.7 & 1.7 & 3.0 \\
\hline & $\mathrm{U}$ & 0.2 & 0.4 & 0.5 \\
\hline & $\mathrm{Am}$ & $<0.1$ & $<0.1$ & $<0.1$ \\
\hline
\end{tabular}


b. $L I X^{\mathrm{TM}}-54$. We diluted $10 \mathrm{~mL}$ of $\mathrm{LIX}^{\mathrm{TM}}-54$ (a beta diketone), obtained from Henkel Corporation, Tucson, AZ, with $20 \mathrm{~mL}$ of cyclohexane and added $26.80 \mathrm{~g}$ of Ambersorb ${ }^{\mathrm{TM}}$ 563 porous carbon beads, obtained from Rohm \& Haas Co., Philadelphia, PA, to absorb the liquid. The cyclohexane evaporated to yield $34.6 \mathrm{~g}$ of the loaded Ambersorb ${ }^{\text {TM }}$ beads.

LIX ${ }^{\text {TM }} 54$ sorbs zirconium slightly from acid-dissolved sludge solution. From acidified supernate solution, the sorption of americium is significantly greater than that of the lanthanides, suggesting that an efficient trivalent actinide/lanthanide group separation is possible. Uranium, iron, manganese, plutonium, and technetium are weakly to moderately sorbed from this solution.

From alkaline supernate solution, this extractant sorbs iron, strontium, yttrium, and manganese at useful levels. Because the $\mathrm{Kd}$ value for yttrium is significantly higher than that for cerium, this extractant might be useful for separating these two elements.

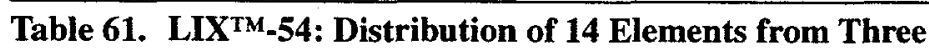

Simulant Solutions for Hanford Tank 102-SY

\begin{tabular}{|c|c|c|c|c|}
\hline \multirow[b]{2}{*}{ Solution } & \multirow[b]{2}{*}{ Element } & \multicolumn{3}{|c|}{ Kd Value for Specified Time } \\
\hline & & $30 \mathrm{~min}$ & $2 \mathrm{~h}$ & $6 \mathrm{~h}$ \\
\hline Acid-Dissolved & $\mathrm{Ce}$ & 0.7 & 0.8 & 0.8 \\
\hline \multirow[t]{13}{*}{ Sludge } & Cs & 0.5 & 0.5 & 0.3 \\
\hline & $\mathrm{Sr}$ & 0.5 & 0.3 & 0.2 \\
\hline & Tc & 0.5 & 0.5 & 0.5 \\
\hline & $Y$ & 0.7 & 0.6 & 0.6 \\
\hline & $\mathrm{Cr}$ & 0.8 & 1.0 & 0.8 \\
\hline & Co & 0.9 & 0.4 & 0.5 \\
\hline & $\mathrm{Fe}$ & 0.7 & 0.3 & 0.4 \\
\hline & Mn & 0.3 & 0.2 & 0.2 \\
\hline & $\mathrm{Zn}$ & 0.5 & 0.3 & 0.2 \\
\hline & $\mathrm{Zr}$ & 1.4 & 1.7 & 3.4 \\
\hline & $\mathrm{U}$ & $<0.1$ & 0.1 & 0.1 \\
\hline & $\mathrm{Pu}$ & 0.5 & 0.1 & 0.1 \\
\hline & $\mathrm{Am}$ & 0.3 & 0.2 & 0.1 \\
\hline Acidified & $\mathrm{Ce}$ & $<0.1$ & 0.4 & 0.4 \\
\hline \multirow[t]{13}{*}{ Supernate } & $\mathrm{Cs}$ & $<0.1$ & 0.3 & 0.1 \\
\hline & $\mathrm{Sr}$ & 0.8 & 0.3 & 0.6 \\
\hline & $\mathrm{Tc}$ & 1.4 & 2.8 & 4.1 \\
\hline & $\mathrm{Y}$ & 0.4 & 0.1 & 0.3 \\
\hline & $\mathrm{Cr}$ & 0.5 & 0.3 & 0.7 \\
\hline & Co & 0.5 & 1.0 & 0.9 \\
\hline & $\mathrm{Fe}$ & 2.9 & 3.4 & 4.7 \\
\hline & $\mathrm{Mn}$ & 2.4 & 2.1 & 2.6 \\
\hline & $\mathrm{Zn}$ & 0.4 & 0.3 & 0.6 \\
\hline & $\mathrm{Zr}$ & 0.1 & 0.3 & 0.4 \\
\hline & $\mathbf{U}$ & 11 & 12 & 12 \\
\hline & $\mathrm{Pu}$ & 2.2 & 2.8 & 3.0 \\
\hline & Am & 34 & 32 & 30 \\
\hline Alkaline & $\mathrm{Ce}$ & 2.3 & 2.5 & 2.9 \\
\hline \multirow[t]{12}{*}{ Supernate } & $\mathrm{Cs}$ & $<0.1$ & $<0.1$ & $<0.1$ \\
\hline & $\mathrm{Sr}$ & 37 & 67 & 84 \\
\hline & $\mathrm{Tc}$ & $<0.1$ & $<0.1$ & 0.2 \\
\hline & $\mathrm{Y}$ & 9.3 & 24 & 56 \\
\hline & $\mathrm{Cr}$ & $<0.1$ & $<0.1$ & 0.2 \\
\hline & Co & 0.3 & 0.6 & 1.2 \\
\hline & $\mathrm{Fe}$ & 41 & 72 & 135 \\
\hline & $\mathrm{Mn}$ & 1.1 & 2.5 & 14 \\
\hline & $\mathrm{Zn}$ & 1.8 & 1.5 & 1.1 \\
\hline & $\mathrm{Zr}$ & 1.2 & 1.5 & 1.7 \\
\hline & $\mathbf{U}$ & $<0.1$ & 0.3 & 0.5 \\
\hline & Am & $<0.1$ & $<0.1$ & 7.4 \\
\hline
\end{tabular}


c. LIX $^{\mathrm{TM}}-84$. We diluted $10 \mathrm{~mL}$ of $\mathrm{LIX}^{\mathrm{TM}}-84$ (a ketoxime), obtained from Henkel Corporation, Tucson, AZ, with $20 \mathrm{~mL}$ of cyclohexane and added $26.13 \mathrm{~g}$ of Ambersorb ${ }^{\mathrm{TM}}$ 563 porous carbon beads, obtained from Rohm \& Haas Co., Philadelphia, PA, to absorb the liquid. The cyclohexane evaporated to yield $34.8 \mathrm{~g}$ of the loaded Ambersorb ${ }^{\text {TM }}$ beads.
LIXTM-84 sorbs none of the 14 elements significantly from acid-dissolved sludge or acidified supernate solutions. From alkaline supernate solution, it sorbs iron, $\mathrm{yttrium}$, and strontium with double-digit $\mathrm{Kd}$ values.

Table 62. LIX'M-84: Distribution of 14 Elements from Three Simulant Solutions for Hanford Tank 102-SY

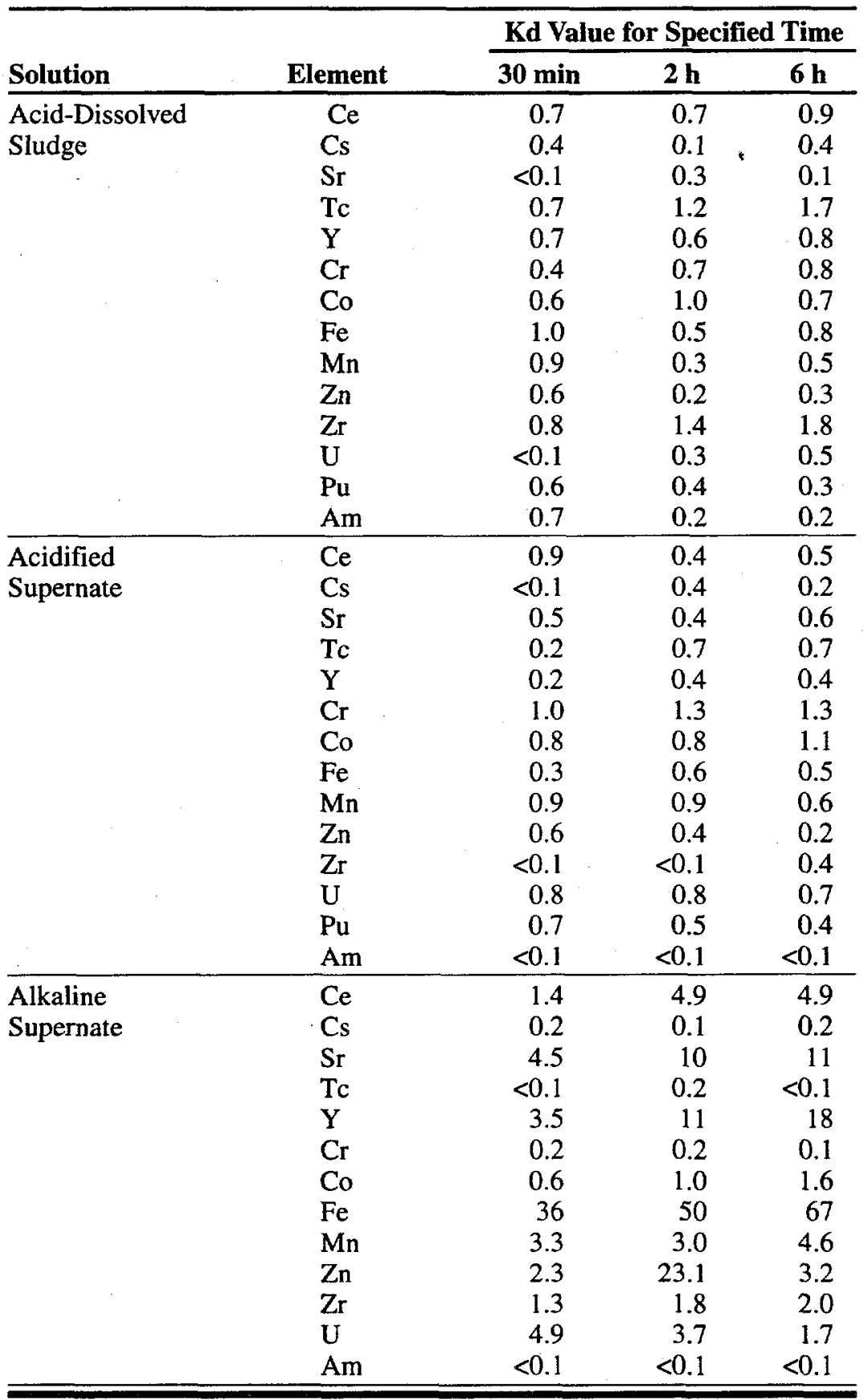


d. $\boldsymbol{L I X}^{\mathrm{TM}}-26$. We diluted $10 \mathrm{~mL}$ of $\mathrm{LIX}^{\mathrm{TM}}-26$ (8-hydroxyquinoline) with $20 \mathrm{~mL}$ of cyclohexane and added $26.90 \mathrm{~g}$ of Ambersorb ${ }^{\mathrm{TM}} 563$ porous carbon beads, obtained from Rohm \& Haas Co., Philadelphia, PA, to absorb the liquid. The cyclohexane evaporated to yield $37.1 \mathrm{~g}$ of the loaded Ambersorb ${ }^{\mathrm{TM}}$ beads.

LIX $^{\mathrm{TM}}-26$ sorbs plutonium moderately and uranium and technetium slightly from acid-dissolved sludge solu- tion. It sorbs technetium, cerium, americium, and uranium at useful levels from alkaline supernate solution. Because the Kd value for cerium is significantly higher than that for yttrium, this extractant is a candidate for separating these two elements. From alkaline supernate solution, it sorbs only technetium with double-digit $\mathrm{Kd}$ values.

Table 63. LIX ${ }^{\mathrm{TM}}$-26: Distribution of 14 Elements from Three Simulant Solutions for Hanford Tank 102-SY

\begin{tabular}{|c|c|c|c|c|}
\hline \multirow[b]{2}{*}{ Solution } & \multirow[b]{2}{*}{ Element } & \multicolumn{3}{|c|}{ Kd Value for Specified Time } \\
\hline & & $30 \mathrm{~min}$ & $2 \mathrm{~h}$ & $6 h$ \\
\hline Acid-Dissolved & $\mathrm{Ce}$ & 0.4 & 0.5 & 0.4 \\
\hline \multirow{13}{*}{ Sludge } & $\mathrm{Cs}$ & 0.4 & 0.5 & 0.2 \\
\hline & $\mathrm{Sr}$ & 0.7 & 0.4 & 0.5 \\
\hline & $\mathrm{Tc}$ & 1.1 & 1.4 & 1.4 \\
\hline & $\mathrm{Y}$ & 0.5 & 0.6 & 0.4 \\
\hline & $\mathrm{Cr}$ & 0.5 & 0.6 & 0.5 \\
\hline & $\mathrm{Co}$ & 0.4 & 0.4 & 0.5 \\
\hline & $\mathrm{Fe}$ & 0.3 & 0.6 & 0.5 \\
\hline & $\mathrm{Mn}$ & $<0.1$ & $<0.1$ & $<0.1$ \\
\hline & $\mathrm{Zn}$ & 0.5 & 0.4 & 0.3 \\
\hline & $\mathrm{Zr}$ & 0.6 & 0.6 & 0.7 \\
\hline & $\mathrm{U}$ & 2.0 & 2.7 & 3.4 \\
\hline & $\mathrm{Pu}$ & 3.9 & 7.9 & 15 \\
\hline & $\mathrm{Am}$ & 0.4 & 0.5 & 0.4 \\
\hline Acidified & $\mathrm{Ce}$ & 32 & 40 & 42 \\
\hline \multirow{13}{*}{ Supernate } & Cs & $<0.1$ & $<0.1$ & $<0.1$ \\
\hline & $\mathrm{Sr}$ & 0.6 & 0.7 & 0.6 \\
\hline & $\mathrm{Tc}$ & 54 & 112 & 190 \\
\hline & $\mathrm{Y}$ & 4.1 & 4.7 & 4.9 \\
\hline & $\mathrm{Cr}$ & $<0.1$ & 0.2 & 0.3 \\
\hline & Co & 1.3 & 1.1 & 1.1 \\
\hline & $\mathrm{Fe}$ & 0.3 & 0.3 & 0.3 \\
\hline & $\mathrm{Mn}$ & $<0.1$ & $<0.1$ & 0.1 \\
\hline & $\mathrm{Zn}$ & 0.3 & 0.2 & 0.1 \\
\hline & $\mathrm{Zr}$ & 0.8 & 0.9 & 0.7 \\
\hline & $\mathrm{U}$ & 12 & 21 & 24 \\
\hline & $\mathrm{Pu}$ & 0.2 & 0.1 & 0.2 \\
\hline & $\mathrm{Am}$ & 24 & 33 & 30 \\
\hline Alkaline & $\mathrm{Ce}$ & 2.5 & 2.7 & 3.6 \\
\hline \multirow{12}{*}{ Supernate } & Cs & $<0.1$ & $<0.1$ & $<0.1$ \\
\hline & $\mathrm{Sr}$ & 2.1 & 3.4 & 5.4 \\
\hline & Tc & 21 & 23 & 22 \\
\hline & $\mathrm{Y}$ & 2.6 & 3.6 & 4.2 \\
\hline & $\mathrm{Cr}$ & 0.2 & 0.2 & 0.4 \\
\hline & Co & $<0.1$ & $<0.1$ & $<0.1$ \\
\hline & $\mathrm{Fe}$ & 0.9 & 0.8 & 0.8 \\
\hline & $\mathrm{Mn}$ & 0.8 & 0.3 & 0.3 \\
\hline & $\mathrm{Zn}$ & 1.1 & 0.6 & 0.5 \\
\hline & $\mathrm{Zr}$ & 0.4 & 0.7 & 0.8 \\
\hline & $\mathrm{U}$ & 1.0 & 0.7 & 0.7 \\
\hline & $\mathrm{Am}$ & $<0.1$ & $<0.1$ & $<0.1$ \\
\hline
\end{tabular}


e. Cyanex ${ }^{\mathrm{TM}}$ 923. We diluted $10 \mathrm{~mL}$ of Cyanex ${ }^{\mathrm{TM}} 923$ (trialkylphosphine oxide), obtained from American Cyanamid Company, Wayne, NJ, with $20 \mathrm{~mL}$ of cyclohexane and added $26.83 \mathrm{~g}$ of Ambersorb ${ }^{\mathrm{TM}} 563$ porous carbon beads, obtained from Rohm \& Haas Co., Philadelphia, PA, to absorb the liquid. The cyclohexane evaporated to yield $36.8 \mathrm{~g}$ of the loaded Ambersorb ${ }^{\mathrm{TM}}$ beads.

Cyanex ${ }^{\mathrm{TM}} 923$ sorbs plutonium, zirconium, and technetium somewhat from acid-dissolved sludge solu- tion but with slow sorption kinetics. However, the plutonium Kd values are an order of magnitude higher when the

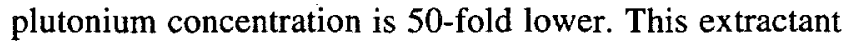
sorbs technetium, lanthanides, americium, and uranium strongly from acidified supernate solution.

From alkaline supernate solution, Cyanex ${ }^{\mathrm{TM}}$ sorbs only technetium well.

Table 64. Cyanex ${ }^{\text {TM }}$ 923: Distribution of 14 Elements from Three

Simulant Solutions for Hanford Tank 102-SY

\begin{tabular}{|c|c|c|c|c|}
\hline \multirow[b]{2}{*}{ Solution } & \multirow[b]{2}{*}{ Element } & \multicolumn{3}{|c|}{ Kd Value for Specified Time } \\
\hline & & $30 \mathrm{~min}$ & $2 \mathrm{~h}$ & $6 \mathrm{~h}$ \\
\hline $\begin{array}{l}\text { Acid-Dissolved } \\
\text { Sludge, } 3 \mathrm{~g} \mathrm{Pu} / \mathrm{L}\end{array}$ & $\begin{array}{l}\mathrm{Ce} \\
\mathrm{Cs} \\
\mathrm{Sr} \\
\mathrm{Tc} \\
\mathrm{Y} \\
\mathrm{Cr} \\
\mathrm{Co} \\
\mathrm{Fe} \\
\mathrm{Mn} \\
\mathrm{Zn} \\
\mathrm{Zr} \\
\mathrm{U} \\
\mathrm{Pu} \\
\mathrm{Am}\end{array}$ & $\begin{array}{r}0.5 \\
0.5 \\
0.8 \\
1.8 \\
0.7 \\
0.8 \\
0.5 \\
<0.1 \\
<0.1 \\
0.2 \\
2.0 \\
<0.1 \\
3.0 \\
0.3\end{array}$ & $\begin{array}{r}0.2 \\
0.3 \\
0.5 \\
2.6 \\
0.4 \\
0.6 \\
0.2 \\
0.4 \\
<0.1 \\
0.1 \\
3.1 \\
1.0 \\
7.6 \\
0.3\end{array}$ & $\begin{array}{r}0.5 \\
0.4 \\
0.5 \\
4.3 \\
0.7 \\
0.9 \\
0.3 \\
0.6 \\
<0.1 \\
0.2 \\
7.6 \\
1.9 \\
21 \\
0.3\end{array}$ \\
\hline $\begin{array}{l}\text { Acid-Dissolved } \\
\text { Sludge, } 60 \mathrm{mg} \mathrm{Pu} / \mathrm{L}\end{array}$ & $\begin{array}{l}\mathrm{U} \\
\mathrm{Pu} \\
\mathrm{Am}\end{array}$ & $\begin{array}{r}2.7 \\
21 \\
0.4\end{array}$ & $\begin{array}{r}6.5 \\
100 \\
0.4 \\
\end{array}$ & $\begin{array}{r}14 \\
199 \\
0.4 \\
\end{array}$ \\
\hline $\begin{array}{l}\text { Acidified } \\
\text { Supernate }\end{array}$ & $\begin{array}{l}\mathrm{Ce} \\
\mathrm{Cs} \\
\mathrm{Sr} \\
\mathrm{Tr} \\
\mathrm{Y} \\
\mathrm{Cr} \\
\mathrm{Co} \\
\mathrm{Fe} \\
\mathrm{Mn} \\
\mathrm{Zn} \\
\mathrm{Zr} \\
\mathrm{U} \\
\mathrm{Pu} \\
\mathrm{Am}\end{array}$ & $\begin{array}{r}744 \\
0.8 \\
0.8 \\
300 \\
1078 \\
<0.1 \\
0.6 \\
<0.1 \\
0.4 \\
2.9 \\
0.9 \\
122 \\
1.4 \\
538 \\
\end{array}$ & $\begin{array}{r}987 \\
0.5 \\
0.5 \\
551 \\
2014 \\
<0.1 \\
0.7 \\
<0.1 \\
0.9 \\
4.4 \\
0.4 \\
233 \\
1.9 \\
751 \\
\end{array}$ & $\begin{array}{r}1596 \\
0.4 \\
0.6 \\
582 \\
2981 \\
0.3 \\
0.7 \\
<0.1 \\
0.7 \\
4.3 \\
0.8 \\
356 \\
2.0 \\
802 \\
\end{array}$ \\
\hline $\begin{array}{l}\text { Alkaline } \\
\text { Supernate }\end{array}$ & $\begin{array}{l}\mathrm{Ce} \\
\mathrm{Cs} \\
\mathrm{Sr} \\
\mathrm{Tc} \\
\mathrm{Y} \\
\mathrm{Cr} \\
\mathrm{Co} \\
\mathrm{Fe} \\
\mathrm{Mn} \\
\mathrm{Zn} \\
\mathrm{Zr} \\
\mathrm{U} \\
\mathrm{Am}\end{array}$ & $\begin{array}{r}1.8 \\
<0.1 \\
1.6 \\
80 \\
1.9 \\
<0.1 \\
<0.1 \\
0.3 \\
0.2 \\
<0.1 \\
0.2 \\
<0.1 \\
<0.1\end{array}$ & $\begin{array}{r}3.8 \\
<0.1 \\
2.6 \\
116 \\
2.9 \\
0.2 \\
0.3 \\
0.4 \\
0.4 \\
0.3 \\
0.6 \\
0.4 \\
<0.1\end{array}$ & $\begin{array}{r}4.5 \\
<0.1 \\
3.8 \\
110 \\
4.1 \\
0.2 \\
0.1 \\
0.7 \\
0.7 \\
0.2 \\
0.6 \\
0.6 \\
<0.1\end{array}$ \\
\hline
\end{tabular}


f. Cyanex $^{\mathrm{TM}}$ 272. We diluted $10 \mathrm{~mL}$ of Cyanex ${ }^{\mathrm{TM}} 272$ (trimethylpentylphosphoric acid), obtained from American Cyanamid Company, Wayne, $\mathrm{NJ}$, with $20 \mathrm{~mL}$ of cyclohexane and added $26.49 \mathrm{~g}$ of Ambersorb ${ }^{\mathrm{TM}} 563$ porous carbon beads, obtained from Rohm \& Haas Co., Philadelphia, PA, to absorb the liquid. The cyclohexane evaporated to yield $37.1 \mathrm{~g}$ of the loaded Ambersorb ${ }^{\mathrm{TM}}$ beads.
Cyanex ${ }^{\mathrm{TM}} 272$ sorbs zirconium strongly and selectively from acid-dissolved sludge solution, although the sorption kinetics are slow. It sorbs uranium selectively at a useful level from acidified supernate solution. However, it sorbs none of the 14 elements studied at even double-digit $\mathrm{Kd}$ values from alkaline supernate solution.

Table 65. Cyanex ${ }^{\mathrm{TM}}$ 272: Distribution of 14 Elements from Three Simulant Solutions for Hanford Tank 102-SY

\begin{tabular}{|c|c|c|c|c|}
\hline \multirow[b]{2}{*}{ Solution } & \multirow[b]{2}{*}{ Element } & \multicolumn{3}{|c|}{ Kd Value for Specified Tim } \\
\hline & & $30 \mathrm{~min}$ & $2 \mathrm{~h}$ & $6 h$ \\
\hline Acid-Dissolved & $\mathrm{Ce}$ & 0.4 & 0.2 & 0.5 \\
\hline \multirow{13}{*}{ Sludge } & $\mathrm{Cs}$ & 0.3 & 0.2 & 0.3 \\
\hline & $\mathrm{Sr}$ & 0.5 & 0.2 & 0.4 \\
\hline & $\mathrm{Tc}$ & 0.4 & 0.6 & 0.7 \\
\hline & $\mathrm{Y}$ & 0.8 & 0.7 & 0.7 \\
\hline & $\mathrm{Cr}$ & 0.5 & 0.6 & 0.8 \\
\hline & Co & 0.5 & 0.4 & 0.4 \\
\hline & $\mathrm{Fe}$ & $<0.1$ & 0.3 & 0.3 \\
\hline & $\mathrm{Mn}$ & $<0.1$ & $<0.1$ & $<0.1$ \\
\hline & $\mathrm{Zn}$ & 0.1 & 0.3 & 0.2 \\
\hline & $\mathrm{Zr}$ & 8.0 & 32 & 199 \\
\hline & $\mathrm{U}$ & 0.3 & 1.4 & 1.4 \\
\hline & $\mathrm{Pu}$ & 0.9 & 1.3 & 2.8 \\
\hline & Am & $<0.1$ & 0.2 & 0.1 \\
\hline Acidified & $\mathrm{Ce}$ & 0.1 & 0.5 & 0.2 \\
\hline \multirow[t]{13}{*}{ Supernate } & Cs & 0.4 & 0.1 & 0.1 \\
\hline & $\mathrm{Sr}$ & 0.9 & 0.6 & 0.9 \\
\hline & $\mathrm{Tc}$ & 0.5 & 0.3 & 0.5 \\
\hline & $\mathrm{Y}$ & 0.8 & 1.0 & 1.1 \\
\hline & $\mathrm{Cr}$ & $<0.1$ & 0.7 & 0.7 \\
\hline & Co & 0.8 & 0.7 & 0.7 \\
\hline & $\mathrm{Fe}$ & 0.5 & 0.6 & 0.6 \\
\hline & Mn & $<0.1$ & $<0.1$ & $<0.1$ \\
\hline & $\mathrm{Zn}$ & 1.1 & 1.3 & 1.6 \\
\hline & $\mathrm{Zr}$ & 0.5 & 0.7 & 0.6 \\
\hline & $\mathrm{U}$ & 23 & 41 & 58 \\
\hline & $\mathrm{Pu}$ & 0.2 & 0.1 & 0.3 \\
\hline & $\mathrm{Am}$ & 1.2 & 1.1 & 1.3 \\
\hline Alkaline & $\mathrm{Ce}$ & 0.5 & 2.9 & 3.6 \\
\hline \multirow{12}{*}{ Supernate } & Cs & $<0.1$ & $<0.1$ & 0.3 \\
\hline & $\mathrm{Sr}$ & 4.0 & 6.3 & 8.5 \\
\hline & Tc & 0.2 & 0.4 & 0.7 \\
\hline & $\mathrm{Y}$ & 1.4 & 2.1 & 3.2 \\
\hline & $\mathrm{Cr}$ & $<0.1$ & 0.2 & 0.4 \\
\hline & Co & $<0.1$ & $<0.1$ & $<0.1$ \\
\hline & $\mathrm{Fe}$ & 0.5 & 0.8 & 1.6 \\
\hline & $\mathrm{Mn}$ & $<0.1$ & $<0.1$ & 0.2 \\
\hline & $\mathrm{Zn}$ & 0.3 & 0.5 & 0.3 \\
\hline & $\mathrm{Zr}$ & 0.4 & 0.6 & 1.2 \\
\hline & $\mathrm{U}$ & $<0.1$ & 0.2 & 0.5 \\
\hline & $\mathrm{Am}$ & $<0.1$ & $<0.1$ & $<0.1$ \\
\hline
\end{tabular}


g. DHDECMP. We diluted $10 \mathrm{~mL}$ of DHDECMP (dihexyl-N,N-diethylcarbamoylmethylphosphonate), obtained from Occidental Chemical Corporation, Specialty Products Division, Niagara Falls, NY, with $20 \mathrm{~mL}$ of cyclohexane and added $27.99 \mathrm{~g}$ of Ambersorb $^{\mathrm{TM}} 563$ porous carbon beads, obtained from Rohm \& Haas Co., Philadelphia, PA, to absorb the liquid. The cyclohexane evaporated to yield $39.5 \mathrm{~g}$ of the loaded Ambersorb ${ }^{\mathrm{TM}}$ beads.
DHDECMP sorbs only plutonium at a useful level from acid-dissolved sludge solution. It sorbs technetium, cerium, americium, and uranium well from acidified supernate solution. A significant separation factor favoring cerium over yttrium also appears in this solution. It sorbs technetium at useful levels from alkaline supernate solution.

Table 66. DHDECMP: Distribution of 14 Elements from Three Simulant Solutions for Hanford Tank 102-SY

\begin{tabular}{|c|c|c|c|c|}
\hline \multirow[b]{2}{*}{ Solution } & \multirow[b]{2}{*}{ Element } & \multicolumn{3}{|c|}{ Kd Value for Specified Time } \\
\hline & & $30 \mathrm{~min}$ & $2 \mathrm{~h}$ & $6 h$ \\
\hline Acid-Dissolved & $\mathrm{Ce}$ & 0.2 & 0.3 & 0.4 \\
\hline \multirow[t]{13}{*}{ Sludge } & $\mathrm{Cs}$ & 0.4 & 0.3 & 0.4 \\
\hline & $\mathrm{Sr}$ & 0.3 & 0.4 & 0.4 \\
\hline & $\mathrm{Tc}$ & 0.7 & 1.0 & 1.6 \\
\hline & $\mathrm{Y}$ & 0.5 & 1.0 & 0.6 \\
\hline & $\mathrm{Cr}$ & 0.8 & 0.8 & 0.7 \\
\hline & $\mathrm{Co}$ & 0.5 & 0.4 & 0.2 \\
\hline & $\mathrm{Fe}$ & 0.2 & 0.3 & 0.3 \\
\hline & $\mathrm{Mn}$ & $<0.1$ & $<0.1$ & $<0.1$ \\
\hline & $\mathrm{Zn}$ & 0.2 & 0.3 & 0.2 \\
\hline & $\mathrm{Zr}$ & 0.9 & 0.6 & 0.9 \\
\hline & $\mathrm{U}$ & 1.3 & 2.2 & 3.2 \\
\hline & $\mathrm{Pu}$ & 4.4 & 8.2 & 15 \\
\hline & Am & 0.4 & 0.6 & 0.4 \\
\hline Acidified & $\mathrm{Ce}$ & 26 & 45 & 51 \\
\hline \multirow[t]{13}{*}{ Supernate } & $\mathrm{Cs}$ & $<0.1$ & $<0.1$ & $<0.1$ \\
\hline & $\mathrm{Sr}$ & 0.5 & 0.6 & 0.7 \\
\hline & $\mathrm{Tc}$ & 43 & 112 & 222 \\
\hline & $\mathrm{Y}$ & 3.4 & 5.1 & 5.8 \\
\hline & $\mathrm{Cr}$ & 0.3 & 0.2 & 0.6 \\
\hline & $\mathrm{Co}$ & 0.8 & 0.9 & 1.1 \\
\hline & $\mathrm{Fe}$ & $<0.1$ & $<0.1$ & $<0.1$ \\
\hline & $\mathrm{Mn}$ & 0.6 & 0.5 & 0.4 \\
\hline & $\mathrm{Zn}$ & 0.7 & 0.4 & 0.5 \\
\hline & $\mathrm{Zr}$ & $<0.1$ & 0.5 & 0.8 \\
\hline & $\mathrm{U}$ & 22 & 31 & 33 \\
\hline & $\mathrm{Pu}$ & 0.4 & 0.3 & 0.4 \\
\hline & Am & 25 & 34 & 35 \\
\hline Alkaline & $\mathrm{Ce}$ & 3.7 & 5.2 & 4.7 \\
\hline \multirow[t]{12}{*}{ Supernate } & $\mathrm{Cs}$ & $<0.1$ & $<0.1$ & $<0.1$ \\
\hline & $\mathrm{Sr}$ & 1.6 & 3.4 & 5.3 \\
\hline & $\mathrm{Tc}$ & 21 & 23 & 21 \\
\hline & $\mathbf{Y}$ & 2.5 & 3.9 & 4.2 \\
\hline & $\mathrm{Cr}$ & 0.2 & 0.2 & 0.3 \\
\hline & $\mathrm{Co}$ & 0.1 & $<0.1$ & $<0.1$ \\
\hline & $\mathrm{Fe}$ & 0.5 & 0.6 & 0.6 \\
\hline & $\mathrm{Mn}$ & $<0.1$ & 0.2 & 0.1 \\
\hline & $\mathrm{Zn}$ & 0.3 & 0.3 & 0.2 \\
\hline & $\mathrm{Zr}$ & 0.7 & 0.8 & 1.1 \\
\hline & $\mathrm{U}$ & 0.3 & 0.4 & 0.5 \\
\hline & Am & $<0.1$ & $<0.1$ & $<0.1$ \\
\hline
\end{tabular}


h. DHDECMP-DIPB. We diluted $10 \mathrm{~mL}$ of DHDECMP, obtained from Occidental Chemical Corporation, Specialty Products Division, Niagara Falls, $\mathrm{NY}$, with $10 \mathrm{~mL}$ of DIPB (diisopropylbenzene) and $30 \mathrm{ml}$ of cyclohexane and added $48.45 \mathrm{~g}$ of AmbersorbTM 563 porous carbon beads, obtained from Rohm \& Haas Co.,
Philadelphia, PA, to absorb the liquid. The cyclohexane evaporated to yield $65.3 \mathrm{~g}$ of the loaded Ambersorb ${ }^{\mathrm{TM}}$ beads.

As expected, the sorption patterns are similar to, but somewhat lower than, those observed with undiluted DHDECMP.

Table 67. DHDECMP-DIPB: Distribution of 14 Elements from Three Simulant Solutions for Hanford HLW 102-SY

\begin{tabular}{|c|c|c|c|c|}
\hline \multirow[b]{2}{*}{ Solution } & \multirow[b]{2}{*}{ Element } & \multicolumn{3}{|c|}{ Kd Value for Specified Time } \\
\hline & & $30 \mathrm{~min}$ & $2 \mathbf{h}$ & $6 \mathrm{~h}$ \\
\hline Acid-Dissolved & $\mathrm{Ce}$ & $<0.1$ & 0.4 & 0.6 \\
\hline \multirow{13}{*}{ Sludge } & Cs & 0.1 & $<0.1$ & 0.2 \\
\hline & $\mathrm{Sr}$ & 0.2 & 0.1 & 0.4 \\
\hline & Tc & 0.6 & 1.1 & 1.5 \\
\hline & $\mathrm{Y}$ & 0.3 & 0.4 & 0.6 \\
\hline & $\mathrm{Cr}$ & 0.5 & 0.7 & 0.9 \\
\hline & Co & 0.1 & 0.4 & 0.5 \\
\hline & $\mathrm{Fe}$ & 0.3 & 0.5 & 0.3 \\
\hline & $\mathrm{Mn}$ & $<0.1$ & $<0.1$ & $<0.1$ \\
\hline & $\mathrm{Zn}$ & 0.3 & 0.5 & 0.2 \\
\hline & $\mathrm{Zr}$ & 0.4 & 0.8 & 1.0 \\
\hline & $\mathrm{U}$ & 1.2 & 2.3 & 2.7 \\
\hline & $\mathrm{Pu}$ & 2.9 & 5.5 & 10 \\
\hline & $\mathrm{Am}$ & 0.4 & 0.6 & 0.3 \\
\hline Acidified & $\mathrm{Ce}$ & 18 & 28 & 28 \\
\hline \multirow[t]{13}{*}{ Supernate } & Cs & 0.1 & $<0.1$ & 0.2 \\
\hline & $\mathrm{Sr}$ & 0.5 & 0.7 & 0.4 \\
\hline & Tc & 27 & 49 & 62 \\
\hline & $\mathrm{Y}$ & 2.9 & 3.3 & 3.1 \\
\hline & $\mathrm{Cr}$ & 0.6 & 0.5 & 0.5 \\
\hline & Co & 0.6 & 0.9 & 0.6 \\
\hline & $\mathrm{Fe}$ & 0.1 & 0.3 & 0.4 \\
\hline & $\mathrm{Mn}$ & $<0.1$ & 0.3 & 0.2 \\
\hline & $\mathrm{Zn}$ & 0.2 & 0.3 & 0.1 \\
\hline & $\mathrm{Zr}$ & 0.4 & 0.5 & 0.5 \\
\hline & $\mathrm{U}$ & 9.3 & 16 & 18 \\
\hline & $\mathrm{Pu}$ & 0.7 & 0.6 & 0.4 \\
\hline & $\mathrm{Am}$ & 14 & 21 & 19 \\
\hline Alkaline & $\mathrm{Ce}$ & 1.3 & 2.4 & 2.6 \\
\hline \multirow[t]{12}{*}{ Supernate } & $\mathrm{Cs}$ & $<0.1$ & $<0.1$ & $<0.1$ \\
\hline & $\mathrm{Sr}$ & 2.3 & 4.0 & 5.9 \\
\hline & $\mathrm{Tc}$ & 14 & 14 & 13 \\
\hline & $\mathrm{Y}$ & 2.2 & 3.5 & 4.4 \\
\hline & $\mathrm{Cr}$ & 0.2 & 0.3 & 0.4 \\
\hline & $\mathrm{Co}$ & $<0.1$ & 0.1 & $<0.1$ \\
\hline & $\mathrm{Fe}$ & 0.8 & 1.4 & 2.3 \\
\hline & $\mathrm{Mn}$ & $<0.1$ & 0.2 & 0.4 \\
\hline & $\mathrm{Zn}$ & 0.5 & 0.7 & 0.8 \\
\hline & $\mathrm{Zr}$ & 0.8 & 1.4 & 1.8 \\
\hline & $\mathrm{U}$ & 0.1 & 0.5 & 0.8 \\
\hline & $\mathrm{Am}$ & $<0.1$ & $<0.1$ & $<0.1$ \\
\hline
\end{tabular}


i. CMPO-DIPB. We diluted $10 \mathrm{~mL}$ of CMPO (octylphenyl-N,N-diisobutylcarbamoylmethylphosphine oxide), obtained from M\&T Chemicals, Inc., Rahway, NJ, with $10 \mathrm{~mL}$ of DIPB (diisopropylbenzene) and $30 \mathrm{ml}$ of cyclohexane and added $46.83 \mathrm{~g}$ of Ambersorb ${ }^{\mathrm{TM}} 563$ porous carbon beads, obtained from Rohm \& Haas Co., Philadelphia, PA, to absorb the liquid. The cyclohexane evaporated to yield $64.1 \mathrm{~g}$ of the loaded Ambersorb ${ }^{T M}$ beads.
CMPO-DIPB sorbs none of the 14 elements at useful levels from acid-dissolved sludge solution. It sorbs technetium, cerium, americium, uranium, and yttrium well from acidified supernate solution, although the sorption kinetics are slow. No elements offer even double-digit $\mathrm{Kd}$ values for sorption from alkaline supernate solution.

Table 68. CMPO-DIPB: Distribution of 14 Elements from Three Simulant Solutions for Hanford Tank 102-SY

\begin{tabular}{|c|c|c|c|c|}
\hline \multirow[b]{2}{*}{ Solution } & \multirow[b]{2}{*}{ Element } & \multicolumn{3}{|c|}{ Kd Value for Specified Time } \\
\hline & & $30 \mathrm{~min}$ & $2 \mathbf{h}$ & $6 \mathrm{~h}$ \\
\hline Acid-Dissolved & $\mathrm{Ce}$ & 0.3 & 0.3 & 0.6 \\
\hline \multirow{13}{*}{ Sludge } & $\mathrm{Cs}$ & 0.5 & 0.2 & 0.3 \\
\hline & $\mathrm{Sr}$ & 0.5 & 0.4 & 0.3 \\
\hline & Tc & 0.6 & 1.1 & 2.2 \\
\hline & $\mathrm{Y}$ & 0.5 & 0.6 & 0.5 \\
\hline & $\mathrm{Cr}$ & 0.7 & 0.8 & 0.9 \\
\hline & Co & 0.2 & 0.1 & 0.1 \\
\hline & $\mathrm{Fe}$ & 0.6 & 0.6 & 0.5 \\
\hline & $\mathrm{Mn}$ & $<0.1$ & $<0.1$ & $<0.1$ \\
\hline & $\mathrm{Zn}$ & 0.4 & 0.3 & 0.3 \\
\hline & $\mathrm{Zr}$ & 0.8 & 0.8 & 1.4 \\
\hline & $\mathbf{U}$ & 0.4 & 0.8 & 1.4 \\
\hline & $\mathrm{Pu}$ & 0.4 & 0.4 & 0.7 \\
\hline & $\mathrm{Am}$ & 0.4 & 0.5 & 0.5 \\
\hline Acidified & $\mathrm{Ce}$ & 78 & 370 & 499 \\
\hline \multirow[t]{13}{*}{ Supernate } & Cs & 0.8 & 0.3 & 0.3 \\
\hline & $\mathrm{Sr}$ & $<0.1$ & 0.2 & 0.3 \\
\hline & $\mathrm{Tc}$ & 41 & 111 & 196 \\
\hline & $\mathrm{Y}$ & 15 & 45 & 89 \\
\hline & $\mathrm{Cr}$ & 0.8 & 0.7 & 0.5 \\
\hline & Co & 0.1 & 1.0 & 0.8 \\
\hline & $\mathrm{Fe}$ & $<0.1$ & $<0.1$ & 0.2 \\
\hline & $\mathrm{Mn}$ & 1.6 & 1.6 & 2.1 \\
\hline & $\mathrm{Zn}$ & 1.0 & 0.4 & 0.5 \\
\hline & $\mathrm{Zr}$ & 1.1 & 0.8 & 0.8 \\
\hline & $\mathrm{U}$ & 49 & 183 & 291 \\
\hline & $\mathrm{Pu}$ & 0.8 & 0.5 & 1.0 \\
\hline & $\mathrm{Am}$ & 49 & 355 & 472 \\
\hline Alkaline & $\mathrm{Ce}$ & 0.4 & 1.0 & 1.3 \\
\hline \multirow[t]{12}{*}{ Supernate } & Cs & 0.2 & $<0.1$ & $<0.1$ \\
\hline & $\mathrm{Sr}$ & 1.9 & 3.4 & 5.0 \\
\hline & Tc & 4.5 & 6.8 & 7.6 \\
\hline & $\mathrm{Y}$ & 1.5 & 3.0 & 4.8 \\
\hline & $\mathrm{Cr}$ & 0.4 & 0.5 & 0.5 \\
\hline & Co & $<0.1$ & 0.1 & 0.2 \\
\hline & $\mathrm{Fe}$ & 0.6 & 0.9 & 1.6 \\
\hline & $\mathrm{Mn}$ & $<0.1$ & $<0.1$ & 0.2 \\
\hline & $\mathrm{Zn}$ & 0.5 & 0.6 & 0.7 \\
\hline & $\mathrm{Zr}$ & 0.7 & 1.2 & 1.8 \\
\hline & $\mathrm{U}$ & 0.4 & 0.6 & 1.0 \\
\hline & $\mathrm{Am}$ & $<0.1$ & $<0.1$ & $<0.1$ \\
\hline
\end{tabular}


j. Aliquat $^{\mathrm{TM}} 336$. We diluted $5.00 \mathrm{~g}$ of Aliquat ${ }^{\mathrm{TM}} 336$ (tricaprylmethylammonium chloride), obtained from Aldrich Chemical Co., with $10 \mathrm{~mL}$ of hexane and added $17.32 \mathrm{~g}$ of Ambersorb ${ }^{\mathrm{TM}} 563$ porous carbon beads, obtained from Rohm \& Haas Co., Philadelphia, PA, to absorb the liquid. The cyclohexane evaporated to yield $26.2 \mathrm{~g}$ of the loaded Ambersorb ${ }^{\mathrm{TM}}$ beads.
Aliquat ${ }^{\mathrm{TM}} 336$ sorbs plutonium moderately from acid-dissolved sludge solution and sorbs technetium strongly and selectively from both acidified supernate and alkaline supernate solutions. The low $\mathrm{Kd}$ value for technetium sorption from acid-dissolved sludge solution indicates that this element might be recovered easily by eluting with nitric acid.

\begin{tabular}{|c|c|c|c|c|}
\hline \multirow[b]{2}{*}{ Solution } & \multirow[b]{2}{*}{ Element } & \multicolumn{3}{|c|}{ Kd Value for Specified Time } \\
\hline & & $30 \mathrm{~min}$ & $2 \mathbf{h}$ & $6 \mathrm{~h}$ \\
\hline $\begin{array}{l}\text { Acid-Dissolved } \\
\text { Sludge }\end{array}$ & $\begin{array}{l}\mathrm{Ce} \\
\mathrm{Cs} \\
\mathrm{Sr} \\
\mathrm{Tc} \\
\mathrm{Y} \\
\mathrm{Cr} \\
\mathrm{Co} \\
\mathrm{Fe} \\
\mathrm{Mn} \\
\mathrm{Zn} \\
\mathrm{Zr} \\
\mathrm{U} \\
\mathrm{Pu} \\
\mathrm{Am}\end{array}$ & $\begin{array}{r}0.4 \\
0.3 \\
0.4 \\
0.7 \\
0.3 \\
<0.1 \\
0.6 \\
0.2 \\
<0.1 \\
0.3 \\
0.8 \\
0.5 \\
2.3 \\
0.2\end{array}$ & $\begin{array}{r}0.6 \\
0.3 \\
0.3 \\
0.8 \\
0.3 \\
<0.1 \\
0.3 \\
0.3 \\
<0.1 \\
0.1 \\
0.4 \\
1.1 \\
5.7 \\
0.2\end{array}$ & $\begin{array}{r}0.6 \\
0.2 \\
0.3 \\
1.0 \\
0.2 \\
0.1 \\
<0.1 \\
0.5 \\
<0.1 \\
0.1 \\
0.6 \\
1.7 \\
14 \\
0.2\end{array}$ \\
\hline $\begin{array}{l}\text { Acidified } \\
\text { Supernate }\end{array}$ & $\begin{array}{l}\mathrm{Ce} \\
\mathrm{Cs} \\
\mathrm{Sr} \\
\mathrm{Tc} \\
\mathrm{Y} \\
\mathrm{Cr} \\
\mathrm{Co} \\
\mathrm{Fe} \\
\mathrm{Mn} \\
\mathrm{Zn} \\
\mathrm{Zr} \\
\mathrm{U} \\
\mathrm{Pu} \\
\mathrm{Am} \\
\end{array}$ & $\begin{array}{r}1.0 \\
1.8 \\
0.3 \\
295 \\
0.3 \\
0.1 \\
0.2 \\
0.2 \\
0.3 \\
<0.1 \\
<0.1 \\
3.2 \\
0.1 \\
0.4 \\
\end{array}$ & $\begin{array}{r}1.6 \\
2.2 \\
0.2 \\
444 \\
0.4 \\
0.1 \\
0.1 \\
0.3 \\
0.3 \\
<0.1 \\
0.1 \\
5.5 \\
0.2 \\
0.6 \\
\end{array}$ & $\begin{array}{r}2.0 \\
2.1 \\
0.3 \\
572 \\
0.2 \\
0.1 \\
<0.1 \\
0.3 \\
0.3 \\
<0.1 \\
0.1 \\
7.9 \\
0.4 \\
0.6 \\
\end{array}$ \\
\hline $\begin{array}{l}\text { Alkaline } \\
\text { Supernate }\end{array}$ & $\begin{array}{l}\mathrm{Ce} \\
\mathrm{Cs} \\
\mathrm{Sr} \\
\mathrm{Tc} \\
\mathrm{Y} \\
\mathrm{Cr} \\
\mathrm{Co} \\
\mathrm{Fe} \\
\mathrm{Mn} \\
\mathrm{Zn} \\
\mathrm{Zr} \\
\mathrm{U} \\
\mathrm{Am}\end{array}$ & $\begin{array}{r}3.7 \\
<0.1 \\
6.8 \\
417 \\
5.2 \\
0.2 \\
<0.1 \\
0.5 \\
0.4 \\
0.2 \\
0.8 \\
0.3 \\
<0.1\end{array}$ & $\begin{array}{r}4.1 \\
<0.1 \\
7.1 \\
614 \\
5.6 \\
0.4 \\
<0.1 \\
1.1 \\
0.6 \\
0.4 \\
1.0 \\
0.5 \\
<0.1\end{array}$ & $\begin{array}{r}5.1 \\
<0.1 \\
8.1 \\
651 \\
6.6 \\
0.7 \\
0.2 \\
1.6 \\
1.1 \\
0.5 \\
1.6 \\
0.7 \\
<0.1\end{array}$ \\
\hline
\end{tabular}


k. Czech Cobalt Dicarbollide. We dissolved $93.6 \mathrm{mg}$ of cobalt dicarbollide, prepared in the Czech Republic, in $2.323 \mathrm{~g}$ of nitrobenzene and $1 \mathrm{~mL}$ of ethanol, and added $2.628 \mathrm{~g}$ of Ambersorb ${ }^{\mathrm{TM}} 563$ porous carbon beads, obtained from Rohm \& Haas Co., Philadelphia, PA, to absorb the liquid. The ethanol evaporated to yield $4.48 \mathrm{~g}$ of the loaded, dry-appearing Ambersorb ${ }^{\mathrm{TM}}$ beads.
This extractant sorbs technetium slightly, with slow sorption kinetics, from acid-dissolved sludge and acidified supernate solution. It was not tested with alkaline supernate solution.

\begin{tabular}{|c|c|c|c|c|}
\hline \multirow[b]{2}{*}{ Solution } & \multirow[b]{2}{*}{ Element } & \multicolumn{3}{|c|}{ Kd Value for Specified Time } \\
\hline & & $30 \mathrm{~min}$ & $\mathbf{2 h}$ & $6 \mathrm{~h}$ \\
\hline $\begin{array}{l}\text { Acid-Dissolved } \\
\text { Sludge }\end{array}$ & $\begin{array}{l}\mathrm{Ce} \\
\mathrm{Cs} \\
\mathrm{Sr} \\
\mathrm{Tc} \\
\mathrm{Y} \\
\mathrm{Cr} \\
\mathrm{Co} \\
\mathrm{Fe} \\
\mathrm{Mn} \\
\mathrm{Zn} \\
\mathrm{Zr} \\
\mathrm{U} \\
\mathrm{Pu} \\
\mathrm{Am}\end{array}$ & $\begin{array}{r}0.5 \\
<0.1 \\
0.6 \\
0.9 \\
0.4 \\
0.2 \\
0.2 \\
<0.1 \\
0.2 \\
0.3 \\
0.5 \\
1.2 \\
<0.1 \\
0.2\end{array}$ & $\begin{array}{r}0.1 \\
<0.1 \\
0.5 \\
2.9 \\
0.4 \\
0.4 \\
0.3 \\
<0.1 \\
0.7 \\
0.3 \\
0.7 \\
0.9 \\
0.3 \\
0.3\end{array}$ & $\begin{array}{r}0.1 \\
0.3 \\
0.9 \\
7.0 \\
0.5 \\
0.4 \\
0.3 \\
<0.1 \\
0.6 \\
0.4 \\
0.8 \\
1.1 \\
0.4 \\
0.3\end{array}$ \\
\hline $\begin{array}{l}\text { Acidified } \\
\text { Supernate }\end{array}$ & $\begin{array}{l}\mathrm{Ce} \\
\mathrm{Cs} \\
\mathrm{Sr} \\
\mathrm{Tc} \\
\mathrm{Y} \\
\mathrm{Cr} \\
\mathrm{Co} \\
\mathrm{Fe} \\
\mathrm{Mn} \\
\mathrm{Zn} \\
\mathrm{Zr} \\
\mathrm{U} \\
\mathrm{Pu} \\
\mathrm{Am}\end{array}$ & $\begin{array}{r}0.7 \\
0.8 \\
<0.1 \\
0.6 \\
<0.1 \\
0.5 \\
0.4 \\
0.2 \\
0.2 \\
0.5 \\
0.2 \\
0.9 \\
0.1 \\
0.2\end{array}$ & $\begin{array}{r}1.4 \\
1.0 \\
0.5 \\
3.8 \\
<0.1 \\
0.9 \\
0.8 \\
<0.1 \\
0.3 \\
0.3 \\
0.7 \\
0.9 \\
<0.1 \\
0.1\end{array}$ & $\begin{array}{r}1.2 \\
0.8 \\
0.7 \\
13 \\
<0.1 \\
1.1 \\
0.7 \\
0.2 \\
0.2 \\
0.2 \\
1.0 \\
0.8 \\
<0.1 \\
0.1\end{array}$ \\
\hline
\end{tabular}


l. Los Alamos Cobalt Dicarbollide. We dissolved $98.1 \mathrm{mg}$ of cobalt dicarbollide, prepared by the Inorganic and Structural Chemistry group (CST-3) at Los Alamos, in $1.816 \mathrm{~g}$ of nitrobenzene and $1 \mathrm{~mL}$ of ethanol. We then added $2.270 \mathrm{~g}$ of Ambersorb ${ }^{\mathrm{TM}} 563$ porous carbon beads, obtained from Rohm \& Haas Co., Philadelphia, PA, to absorb the liquid. The ethanol evaporated to yield $3.64 \mathrm{~g}$ of the loaded, dry-appearing Ambersorb ${ }^{\text {TM }}$ beads.

This extractant sorbs technetium slightly, with slow sorption kinetics, from acid-dissolved sludge and acidified supernate solution. It was not tested with alkaline supernate solution.

Table 71. Los Alamos Cobalt Dicarbollide: Distribution of 14

Elements from Two Simulant Solutions for Hanford Tank 102-SY

\begin{tabular}{lcrrr}
\hline & & \multicolumn{3}{c}{ Kd Value for Specified Time } \\
\cline { 2 - 5 } Solution & Element & $\mathbf{3 0} \mathbf{~ m i n}$ & $\mathbf{2 h}$ & $\mathbf{6 h}$ \\
\hline Acid-Dissolved & $\mathrm{Ce}$ & $<0.1$ & $<0.1$ & $<0.1$ \\
Sludge & $\mathrm{Cs}$ & 1.5 & 1.8 & 1.2 \\
& $\mathrm{Sr}$ & 0.6 & 0.5 & 0.6 \\
& $\mathrm{Tc}$ & 1.6 & 3.4 & 7.7 \\
& $\mathrm{Y}$ & 0.6 & 0.2 & 0.2 \\
& $\mathrm{Cr}$ & 0.1 & $<0.1$ & 0.1 \\
& $\mathrm{Co}$ & 0.2 & 0.5 & 0.2 \\
& $\mathrm{Fe}$ & 0.1 & $<0.1$ & $<0.1$ \\
& $\mathrm{Mn}$ & $<0.1$ & 0.2 & 0.3 \\
& $\mathrm{Zn}$ & 0.2 & 0.3 & 0.3 \\
& $\mathrm{Zr}$ & 0.4 & 0.4 & 0.7 \\
& $\mathrm{U}$ & 0.7 & 0.7 & 0.5 \\
& $\mathrm{Pu}$ & $<0.1$ & $<0.1$ & 0.2 \\
& $\mathrm{Am}$ & 0.1 & 0.1 & 0.2 \\
\hline Acidified & $\mathrm{Ce}$ & 0.9 & 0.8 & 0.6 \\
Supernate & $\mathrm{Cs}$ & 3.1 & 2.0 & 1.2 \\
& $\mathrm{Sr}$ & 0.6 & 0.5 & 0.2 \\
& $\mathrm{Tc}$ & 1.9 & 7.2 & 44 \\
& $\mathrm{Y}$ & $<0.1$ & $<0.1$ & $<0.1$ \\
& $\mathrm{Cr}$ & 0.8 & 1.2 & 0.6 \\
& $\mathrm{Co}$ & 0.6 & 1.0 & 0.5 \\
& $\mathrm{Fe}$ & 0.2 & 0.5 & 0.4 \\
& $\mathrm{Mn}$ & 0.2 & 0.1 & 0.1 \\
& $\mathrm{Zn}$ & $<0.1$ & 0.1 & 0.1 \\
& $\mathrm{Zr}$ & 1.1 & 0.9 & 0.8 \\
& $\mathrm{U}$ & 0.5 & 0.9 & 0.9 \\
& $\mathrm{Pu}$ & 0.3 & 0.1 & 0.2 \\
& $\mathrm{Am}$ & $<0.1$ & 0.1 & 0.1 \\
\hline
\end{tabular}


7. Sandia Absorbers. Materials designated as SNL/ CST are a new class of inorganic ion exchangers called crystalline silico-titanates (CSTs). These materials, jointly invented by Sandia National Laboratories and Texas A\&M University (a patent application has been filed), show a significant potential for removing cesium from defense wastes that contain more than $5 \mathrm{M} \mathrm{Na}^{+}$and more than $1 \mathrm{M}$ $\mathrm{OH}^{-}$. CST materials used in this study are very fine powders composed of cuboidal crystals with particle sizes of several hundred Angstroms. Different numerical values in the absorber designations represent different synthesis procedures.

Because the composition of the various CST materials we evaluated is proprietary, we can provide only general information. SNL/CST 35, 48, 69, and 84 are developmental compositions that were prepared in relatively small quantities. As the development effort progressed, however, a baseline composition was selected for larger-scale synthesis, characterization, and evaluation of properties. SNL/CST 111, 120, 141, and 149 are baseline materials that are being developed for the removal of cesium and other radionuclides from a wide range of waste solutions.

SNL/CST 141 preparation has been demonstrated at a pilot-plant scale. The CST synthesis procedures currently are being scaled up for commercial production by UOP Corporation, Des Plaines, IL, Sandia's partner in a cooperative research and development agreement (CRADA). An engineered form of CST suitable for use in ion exchange columns is scheduled to be commercially available in 1995 .
During earlier screening studies conducted at Sandia, the selectivities of these materials for removing cesium were determined. Using $100 \mathrm{ppm}$ of cesium in a simulant of $5.7 \mathrm{M} \mathrm{Na}^{+}$and $0.6 \mathrm{M} \mathrm{OH}^{-}$, the cesium selectivities were SNL/CST $35=$ SNL/CST $120<$ SNL/ CST $48<$ SNL/CST $68<$ SNL/CST $69<$ SNL/CST 84 < SNL/CST $149<$ SNL/CST $111<$ SNL/CST 141.

As discussed in section II.D, unusually large variations in $\mathrm{Kd}$ values obtained with CST absorbers may indicate that submicrometer-sized CST particles at times contaminated the solution taken for gamma spectrometric assay. Inadvertent introduction of particles into the assay solution would, however, always cause us to understate the actual Kd values for the sorption of cesium from solution. Our reported $\mathrm{Kd}$ values for cesium sorption on the CST materials, therefore, represent minimum values.

In a few cases, the $\mathrm{Kd}$ values for non-cesium elements decrease with increasing contact time. Sandia personnel who prepared the CSTs suggested that this could be caused by a small amount of an impurity that preferentially sorbs certain non-cesium elements. However, because the proposed impurity slowly dissolves out of the crystalline material, some of the initially sorbed non-cesium element would be released, causing the $\mathrm{Kd}$ value to decrease with increased contact time. Displacement by a more strongly sorbed, but kinetically slower, ionic species is another possibility. 
a. SNL/CST 35 Crystalline Silico-Titanate. This absorber strongly and quite selectively sorbs cesium from acid-dissolved sludge solution; only zirconium is cosorbed to a significant extent. Cesium sorbs even more strongly from acidified supernate solution, although uranium also sorbs at useful levels from this solution.
SNL/CST 35 sorbs cesium well and strontium strongly from alkaline supernate solution, and many other elements are cosorbed at moderate to high levels. The fact that only cesium is strongly sorbed from acid-dissolved sludge solution indicates that most non-cesium elements sorbed from alkaline solution could subsequently be eluted with nitric acid, leaving cesium selectively sorbed.

\begin{tabular}{|c|c|c|c|c|}
\hline \multirow[b]{2}{*}{ Solution } & \multirow[b]{2}{*}{ Element } & \multicolumn{3}{|c|}{ Kd Value for Specified Time } \\
\hline & & $30 \mathrm{~min}$ & $2 \mathrm{~h}$ & $6 \mathrm{~h}$ \\
\hline $\begin{array}{l}\text { Acid-Dissolved } \\
\text { Sludge }\end{array}$ & $\begin{array}{l}\mathrm{Ce} \\
\mathrm{Cs} \\
\mathrm{Sr} \\
\mathrm{Tc} \\
\mathrm{Y} \\
\mathrm{Cr} \\
\mathrm{Co} \\
\mathrm{Fe} \\
\mathrm{Mn} \\
\mathrm{Zn} \\
\mathrm{Zr} \\
\mathrm{U} \\
\mathrm{Pu} \\
\mathrm{Am} \\
\end{array}$ & $\begin{array}{r}0.6 \\
4485 \\
<0.1 \\
0.9 \\
0.3 \\
0.4 \\
0.6 \\
0.8 \\
<0.1 \\
0.5 \\
8.3 \\
0.9 \\
2.4 \\
<0.1 \\
\end{array}$ & $\begin{array}{r}0.7 \\
1011 \\
<0.1 \\
0.5 \\
0.4 \\
0.4 \\
0.7 \\
0.5 \\
<0.1 \\
0.3 \\
26 \\
0.5 \\
2.6 \\
0.1 \\
\end{array}$ & $\begin{array}{r}0.7 \\
3416 \\
<0.1 \\
0.5 \\
0.4 \\
0.4 \\
0.5 \\
0.8 \\
0.1 \\
0.3 \\
76 \\
0.5 \\
2.4 \\
<0.1 \\
\end{array}$ \\
\hline $\begin{array}{l}\text { Acidified } \\
\text { Supernate }\end{array}$ & $\begin{array}{l}\mathrm{Ce} \\
\mathrm{Cs} \\
\mathrm{Sr} \\
\mathrm{Tc} \\
\mathrm{Y} \\
\mathrm{Cr} \\
\mathrm{Co} \\
\mathrm{Fe} \\
\mathrm{Mn} \\
\mathrm{Zn} \\
\mathrm{Zr} \\
\mathrm{U} \\
\mathrm{Pu} \\
\mathrm{Am} \\
\end{array}$ & $\begin{array}{r}2.9 \\
>13 \mathrm{~K} \\
1.6 \\
1.2 \\
2.5 \\
1.1 \\
0.7 \\
0.9 \\
0.5 \\
1.5 \\
0.5 \\
25 \\
0.8 \\
1.3 \\
\end{array}$ & $\begin{array}{r}2.1 \\
>12 \mathrm{~K} \\
1.3 \\
1.7 \\
1.7 \\
1.9 \\
0.2 \\
2.2 \\
0.1 \\
1.1 \\
1.1 \\
45 \\
1.5 \\
1.1 \\
\end{array}$ & $\begin{array}{r}6.7 \\
4195 \\
3.3 \\
5.6 \\
0.9 \\
9.6 \\
0.2 \\
2.5 \\
0.1 \\
0.9 \\
3.6 \\
54 \\
1.5 \\
0.7 \\
\end{array}$ \\
\hline $\begin{array}{l}\text { Alkaline } \\
\text { Supernate }\end{array}$ & $\begin{array}{l}\mathrm{Ce} \\
\mathrm{Cs} \\
\mathrm{Sr} \\
\mathrm{Tc} \\
\mathrm{Y} \\
\mathrm{Cr} \\
\mathrm{Co} \\
\mathrm{Fe} \\
\mathrm{Mn} \\
\mathrm{Zn} \\
\mathrm{Zr} \\
\mathrm{U} \\
\mathrm{Am}\end{array}$ & $\begin{array}{r}153 \\
165 \\
>4200 \\
0.6 \\
8.9 \\
0.5 \\
0.5 \\
55 \\
95 \\
6.9 \\
113 \\
31 \\
63\end{array}$ & $\begin{array}{r}>300 \\
277 \\
>4400 \\
0.2 \\
17 \\
0.2 \\
0.5 \\
113 \\
375 \\
9.0 \\
280 \\
56 \\
123\end{array}$ & $\begin{array}{r}191 \\
385 \\
>4600 \\
0.2 \\
37 \\
0.2 \\
0.5 \\
181 \\
635 \\
10 \\
504 \\
72 \\
138\end{array}$ \\
\hline
\end{tabular}


b. SNL/CST 48 Crystalline Silico-Titanate. This absorber strongly sorbs cesium from acid-dissolved sludge solution; only zirconium is cosorbed to a significant extent. It sorbs cesium even more strongly from acidified supernate solution, although uranium, zinc, cerium and americium are cosorbed with double-digit Kd values. SNL/CST 48 was not studied with alkaline supernate solution.

\begin{tabular}{|c|c|c|c|c|}
\hline \multirow[b]{2}{*}{ Solution } & \multirow[b]{2}{*}{ Element } & \multicolumn{3}{|c|}{ Kd Value for Specified Time } \\
\hline & & $30 \mathrm{~min}$ & $2 \mathbf{h}$ & $6 \mathrm{~h}$ \\
\hline $\begin{array}{l}\text { Acid-Dissolved } \\
\text { Sludge }\end{array}$ & $\begin{array}{l}\mathrm{Ce} \\
\mathrm{Cs} \\
\mathrm{Sr} \\
\mathrm{Tc} \\
\mathrm{Y} \\
\mathrm{Cr} \\
\mathrm{Co} \\
\mathrm{Fe} \\
\mathrm{Mn} \\
\mathrm{Zn} \\
\mathrm{Zr} \\
\mathrm{U} \\
\mathrm{Pu} \\
\mathrm{Am} \\
\end{array}$ & $\begin{array}{r}0.6 \\
794 \\
<0.1 \\
0.8 \\
0.7 \\
0.3 \\
0.9 \\
0.7 \\
<0.1 \\
0.3 \\
15 \\
1.5 \\
2.6 \\
<0.1 \\
\end{array}$ & $\begin{array}{r}0.4 \\
758 \\
<0.1 \\
0.5 \\
0.4 \\
0.3 \\
0.4 \\
1.0 \\
<0.1 \\
0.2 \\
49 \\
1.1 \\
3.2 \\
0.2 \\
\end{array}$ & $\begin{array}{r}0.6 \\
1180 \\
<0.1 \\
0.6 \\
0.5 \\
0.3 \\
0.6 \\
1.0 \\
<0.1 \\
0.2 \\
51 \\
1.2 \\
4.4 \\
0.1 \\
\end{array}$ \\
\hline $\begin{array}{l}\text { Acidified } \\
\text { Supernate }\end{array}$ & $\begin{array}{l}\mathrm{Ce} \\
\mathrm{Cs} \\
\mathrm{Sr} \\
\mathrm{Tc} \\
\mathrm{Y} \\
\mathrm{Cr} \\
\mathrm{Co} \\
\mathrm{Fe} \\
\mathrm{Mn} \\
\mathrm{Zn} \\
\mathrm{Zr} \\
\mathrm{U} \\
\mathrm{Pu} \\
\mathrm{Am}\end{array}$ & $\begin{array}{r}6.4 \\
3951 \\
1.8 \\
1.6 \\
6.0 \\
1.3 \\
4.5 \\
1.2 \\
2.0 \\
23 \\
0.7 \\
16 \\
<0.1 \\
9.4\end{array}$ & $\begin{array}{r}8.6 \\
2820 \\
1.2 \\
1.5 \\
7.0 \\
1.7 \\
4.6 \\
1.5 \\
1.7 \\
32 \\
0.8 \\
30 \\
<0.1 \\
13\end{array}$ & $\begin{array}{r}17 \\
1030 \\
1.2 \\
2.0 \\
5.3 \\
6.0 \\
2.0 \\
1.8 \\
1.2 \\
34 \\
0.8 \\
37 \\
0.1 \\
15\end{array}$ \\
\hline
\end{tabular}


c. SNL/CST 68 Crystalline Silico-Titanate. This absorber strongly sorbs cesium from acid-dissolved sludge solution; only zirconium is cosorbed at useful levels. It sorbs cesium even more strongly from acidified supernate solution, although uranium, zinc, cerium, and americium are cosorbed with double-digit $\mathrm{Kd}$ values. The decreasing $\mathrm{Kd}$ values for cesium may be real, or they may reflect incomplete filtration of the submicrometer-sized absorber particles from the assay solutions. SNL/CST 68 was not studied with alkaline supernate solution.

\begin{tabular}{|c|c|c|c|c|}
\hline \multirow{2}{*}{ Solution } & \multirow{2}{*}{ Element } & \multicolumn{3}{|c|}{ Kd Value for Specified Time } \\
\hline & & $30 \mathrm{~min}$ & $2 \mathrm{~h}$ & $6 \mathrm{~h}$ \\
\hline $\begin{array}{l}\text { Acid-Dissolved } \\
\text { Sludge }\end{array}$ & $\begin{array}{l}\mathrm{Ce} \\
\mathrm{Cs} \\
\mathrm{Sr} \\
\mathrm{Tc} \\
\mathrm{Y} \\
\mathrm{Cr} \\
\mathrm{Co} \\
\mathrm{Fe} \\
\mathrm{Mn} \\
\mathrm{Zn} \\
\mathrm{Zr} \\
\mathrm{U} \\
\mathrm{Pu} \\
\mathrm{Am} \\
\end{array}$ & $\begin{array}{r}0.1 \\
688 \\
<0.1 \\
0.6 \\
0.5 \\
0.1 \\
0.5 \\
0.7 \\
<0.1 \\
0.2 \\
31 \\
1.1 \\
3.9 \\
0.4 \\
\end{array}$ & $\begin{array}{r}0.4 \\
2308 \\
<0.1 \\
0.4 \\
0.2 \\
0.4 \\
0.3 \\
0.7 \\
0.3 \\
0.4 \\
119 \\
1.2 \\
4.4 \\
0.1 \\
\end{array}$ & $\begin{array}{r}0.7 \\
1049 \\
<0.1 \\
0.8 \\
0.3 \\
0.4 \\
0.5 \\
0.9 \\
0.1 \\
0.3 \\
103 \\
1.0 \\
5.3 \\
0.1 \\
\end{array}$ \\
\hline $\begin{array}{l}\text { Acidified } \\
\text { Supernate }\end{array}$ & $\begin{array}{l}\mathrm{Ce} \\
\mathrm{Cs} \\
\mathrm{Sr} \\
\mathrm{Tc} \\
\mathrm{Y} \\
\mathrm{Cr} \\
\mathrm{Co} \\
\mathrm{Fe} \\
\mathrm{Mn} \\
\mathrm{Zn} \\
\mathrm{Zr} \\
\mathrm{U} \\
\mathrm{Pu} \\
\mathrm{Am}\end{array}$ & $\begin{array}{r}8.6 \\
4072 \\
1.7 \\
1.5 \\
7.4 \\
1.7 \\
5.3 \\
0.9 \\
1.9 \\
33 \\
1.0 \\
23 \\
0.6 \\
15\end{array}$ & $\begin{array}{r}16 \\
2453 \\
1.6 \\
1.8 \\
9.7 \\
2.4 \\
5.4 \\
1.7 \\
1.9 \\
44 \\
1.2 \\
38 \\
0.5 \\
20\end{array}$ & $\begin{array}{r}38 \\
722 \\
1.3 \\
1.7 \\
7.5 \\
6.6 \\
2.3 \\
2.2 \\
1.6 \\
43 \\
0.9 \\
45 \\
0.3 \\
20\end{array}$ \\
\hline
\end{tabular}


d. SNL/CST 69 Crystalline Silico-Titanate. This absorber strongly sorbs cesium from acid-dissolved sludge solution; only zirconium is cosorbed at useful levels. It sorbs cesium even more strongly from acidified supernate solution, although uranium, chromium, cerium, and technetium are cosorbed with double-digit Kd values. Sorption of cesium from alkaline supernate solution is much lower, whereas strontium, cerium, manganese, iron, zirconium, and americium are strongly sorbed from this solution.

Table 75. SNL/CST 69: Distribution of 14 Elements from Three Simulant Solutions for Hanford Tank 102-SY

\begin{tabular}{|c|c|c|c|c|}
\hline \multirow[b]{2}{*}{ Solution } & \multirow[b]{2}{*}{ Element } & \multicolumn{3}{|c|}{ Kd Value for Specified Time } \\
\hline & & $30 \mathrm{~min}$ & $2 \mathrm{~h}$ & $6 \mathrm{~h}$ \\
\hline \multirow{14}{*}{$\begin{array}{l}\text { Acid-Dissolved } \\
\text { Sludge }\end{array}$} & $\mathrm{Ce}$ & 0.5 & 0.6 & 0.7 \\
\hline & Cs & 2884 & 2279 & 3252 \\
\hline & $\mathrm{Sr}$ & $<0.1$ & $<0.1$ & $<0.1$ \\
\hline & Tc & 1.1 & 0.8 & 1.1 \\
\hline & $\mathrm{Y}$ & 0.4 & 0.4 & 0.2 \\
\hline & $\mathrm{Cr}$ & 0.6 & 0.7 & 0.6 \\
\hline & Co & 0.4 & 0.4 & 0.4 \\
\hline & $\mathrm{Fe}$ & 0.4 & 0.6 & 0.7 \\
\hline & $\mathrm{Mn}$ & $<0.1$ & $<0.1$ & $<0.1$ \\
\hline & $\mathrm{Zn}$ & 0.4 & 0.4 & 0.4 \\
\hline & $\mathrm{Zr}$ & 91 & - & 115 \\
\hline & $\mathrm{U}$ & 0.7 & 1.1 & 1.2 \\
\hline & $\mathrm{Pu}$ & 3.4 & 3.8 & 4.5 \\
\hline & $\mathrm{Am}$ & 0.6 & 0.3 & 0.3 \\
\hline Acidified & $\mathrm{Ce}$ & 4.0 & 4.2 & 14 \\
\hline \multirow{13}{*}{ Supernate } & Cs & $>8 \mathrm{~K}$ & $>10 \mathrm{~K}$ & $>20 \mathrm{~K}$ \\
\hline & $\mathrm{Sr}$ & 3.4 & 2.9 & 6.6 \\
\hline & $\mathrm{Tc}$ & 4.9 & 6.7 & 12 \\
\hline & $\mathrm{Y}$ & 1.8 & 1.0 & 1.6 \\
\hline & $\mathrm{Cr}$ & 2.8 & 3.8 & 14 \\
\hline & Co & $<0.1$ & $<0.1$ & $<0.1$ \\
\hline & $\mathrm{Fe}$ & 2.2 & 2.0 & 2.2 \\
\hline & $\mathbf{M n}$ & 0.4 & 0.4 & 0.4 \\
\hline & $\mathrm{Zn}$ & 2.0 & 2.9 & 3.7 \\
\hline & $\mathrm{Zr}$ & 1.7 & 2.5 & 5.5 \\
\hline & $\mathbf{U}$ & 21 & 33 & 43 \\
\hline & $\mathrm{Pu}$ & 0.7 & 0.7 & 1.0 \\
\hline & $\mathrm{Am}$ & 2.5 & 3.0 & 3.3 \\
\hline Alkaline & $\mathrm{Ce}$ & 205 & $>290$ & 298 \\
\hline \multirow{12}{*}{ Supernate } & Cs & 10 & 13 & 16 \\
\hline & $\mathrm{Sr}$ & 3839 & 1332 & 1659 \\
\hline & Tc & 0.4 & $<0.1$ & 1.3 \\
\hline & $\mathrm{Y}$ & 16 & 26 & 38 \\
\hline & $\mathrm{Cr}$ & $<0.1$ & $<0.1$ & 1.2 \\
\hline & Co & 0.6 & 0.4 & 1.0 \\
\hline & $\mathrm{Fe}$ & 45 & 80 & 145 \\
\hline & $\mathbf{M n}$ & 50 & 101 & 167 \\
\hline & $\mathrm{Zn}$ & 3.8 & 4.9 & 5.4 \\
\hline & $\mathrm{Zr}$ & 32 & 87 & 141 \\
\hline & $\mathrm{U}$ & 6.7 & 9.3 & 10 \\
\hline & $\mathrm{Am}$ & 24 & 67 & 135 \\
\hline
\end{tabular}


e. SNL/CST 84 Crystalline Silico-Titanate. This absorber strongly sorbs cesium from acid-dissolved sludge solution; only zirconium is cosorbed at useful levels. The fact that $\mathrm{Kd}$ values for zirconium sorption from acid-dissolved sludge solution decrease, while those for cesium increase, may reflect slow dissolution of an amorphous phase that preferentially sorbs zirconium.
This absorber sorbs cesium well from acidified supernate solution, although uranium and cerium are cosorbed with double-digit values. SNL/CST 84 was not studied with alkaline supernate solution.

Table 76. SNL/CST 84: Distribution of 14 Elements from Two

Simulant Solutions for Hanford Tank 102-SY

\begin{tabular}{lcrrr}
\hline & & \multicolumn{3}{c}{ Kd Value for Specified Time } \\
\cline { 2 - 5 } Solution & Element & $\mathbf{3 0} \mathbf{~ m i n}$ & $\mathbf{2 ~ h}$ & $\mathbf{6 ~ h}$ \\
\hline Acid-Dissolved & $\mathrm{Ce}$ & 0.4 & 0.1 & 0.5 \\
Sludge & $\mathrm{Cs}$ & $\mathbf{1 8 4 9}$ & $>8 \mathrm{~K}$ & $>11 \mathrm{~K}$ \\
& $\mathrm{Sr}$ & $<0.1$ & $<0.1$ & $<0.1$ \\
& $\mathrm{Tc}$ & 0.8 & 0.4 & 0.7 \\
& $\mathrm{Y}$ & $<0.1$ & 0.1 & 0.2 \\
& $\mathrm{Cr}$ & 0.4 & 0.6 & 0.6 \\
& $\mathrm{Co}$ & 0.6 & 0.3 & 0.4 \\
& $\mathrm{Fe}$ & 0.4 & 0.2 & 0.4 \\
& $\mathrm{Mn}$ & $<0.1$ & 0.1 & $<0.1$ \\
& $\mathrm{Zn}$ & 0.4 & 0.3 & 0.2 \\
& $\mathrm{Zr}$ & 70 & 87 & 19 \\
& $\mathrm{U}$ & 0.6 & 0.9 & 0.5 \\
& $\mathrm{Pu}$ & 2.0 & 1.8 & 1.9 \\
& $\mathrm{Am}$ & 0.1 & $<0.1$ & $<0.1$ \\
\hline Acidified & $\mathrm{Ce}$ & 3.5 & 4.8 & 14 \\
Supernate & $\mathrm{Cs}$ & 5484 & 3953 & 3098 \\
& $\mathrm{Sr}$ & 2.2 & 2.0 & 4.4 \\
& $\mathrm{Tc}$ & 1.5 & 1.9 & 4.6 \\
& $\mathrm{Y}$ & 1.3 & 1.1 & 1.1 \\
& $\mathrm{Cr}$ & 1.4 & 1.7 & 8.8 \\
& $\mathrm{Co}$ & 0.3 & 0.2 & 0.1 \\
& $\mathrm{Fe}$ & 0.6 & 1.2 & 1.4 \\
& $\mathrm{Mn}$ & 0.9 & 0.6 & 0.5 \\
& $\mathrm{Zn}$ & 1.0 & 1.4 & 2.1 \\
& $\mathrm{Zr}$ & 0.9 & 1.1 & 3.0 \\
& $\mathrm{U}$ & 13 & 19 & 22 \\
& $\mathrm{Pu}$ & 0.1 & 0.3 & 0.2 \\
& $\mathrm{Am}$ & 2.9 & 4.1 & 5.8 \\
\hline \hline
\end{tabular}


f. SNL/CST 111 Crystalline Silico-Titanate. This absorber strongly sorbs cesium from acid-dissolved sludge solution; only zirconium is cosorbed to a significant extent. It sorbs cesium well from acidified supernate solution, although americium, cerium, and uranium are cosorbed at useful levels. The decreasing $\mathrm{Kd}$ values for cesium sorption from basic solution may be real or may reflect incomplete filtration of the submicrometer-sized absorber particles from the assay solutions.
SNL/CST 111 strongly sorbs cesium and strontium from alkaline supernate solution, whereas many other elements are cosorbed at moderate to high levels. The fact that only cesium is strongly sorbed from aciddissolved sludge solution indicates that most non-cesium elements sorbed from alkaline solution could subsequently be eluted with nitric acid, leaving cesium selectively sorbed.

\begin{tabular}{|c|c|c|c|c|}
\hline \multirow[b]{2}{*}{ Solution } & \multirow[b]{2}{*}{ Element } & \multicolumn{3}{|c|}{ Kd Value for Specified Time } \\
\hline & & $30 \mathrm{~min}$ & $2 \mathrm{~h}$ & $6 \mathrm{~h}$ \\
\hline $\begin{array}{l}\text { Acid-Dissolved } \\
\text { Sludge }\end{array}$ & $\begin{array}{l}\mathrm{Ce} \\
\mathrm{Cs} \\
\mathrm{Sr} \\
\mathrm{T} c \\
Y \\
\mathrm{Cr} \\
\mathrm{C} 0 \\
\mathrm{Fe} \\
\mathrm{Mn} \\
\mathrm{Zn} \\
\mathrm{Zr} \\
\mathrm{U} \\
\mathrm{Pu} \\
\mathrm{Am}\end{array}$ & $\begin{array}{r}1.0 \\
3420 \\
0.6 \\
0.6 \\
0.3 \\
1.0 \\
<0.1 \\
0.6 \\
0.1 \\
0.8 \\
11 \\
0.7 \\
3.1 \\
0.2\end{array}$ & $\begin{array}{r}0.9 \\
>5 \mathrm{~K} \\
0.4 \\
0.7 \\
0.3 \\
1.1 \\
<0.1 \\
0.6 \\
0.3 \\
0.6 \\
14 \\
1.3 \\
3.5 \\
0.3\end{array}$ & $\begin{array}{r}1.0 \\
>5 \mathrm{~K} \\
0.5 \\
0.7 \\
0.3 \\
1.2 \\
<0.1 \\
0.7 \\
0.1 \\
0.6 \\
18 \\
1.5 \\
3.8 \\
0.3\end{array}$ \\
\hline $\begin{array}{l}\text { Acidified } \\
\text { Supernate }\end{array}$ & $\begin{array}{l}\mathrm{Ce} \\
\mathrm{C} s \\
\mathrm{Sr} \\
\mathrm{T} c \\
\mathrm{Y} \\
\mathrm{Cr} \\
\mathrm{C} 0 \\
\mathrm{Fe} \\
\mathrm{Mn} \\
\mathrm{Zn} \\
\mathrm{Zr} \\
\mathrm{U} \\
\mathrm{Pu} \\
\mathrm{Am}\end{array}$ & $\begin{array}{r}17 \\
4939 \\
4.2 \\
1.5 \\
4.4 \\
2.1 \\
1.0 \\
0.6 \\
1.2 \\
2.3 \\
0.9 \\
15 \\
0.2 \\
25\end{array}$ & $\begin{array}{r}32 \\
2713 \\
4.2 \\
1.8 \\
4.7 \\
1.9 \\
0.8 \\
1.6 \\
1.6 \\
3.2 \\
1.0 \\
21 \\
0.8 \\
52\end{array}$ & $\begin{array}{r}57 \\
1864 \\
3.5 \\
1.2 \\
4.8 \\
1.7 \\
0.4 \\
1.9 \\
1.5 \\
5.1 \\
0.6 \\
25 \\
0.4 \\
107\end{array}$ \\
\hline $\begin{array}{l}\text { Alkaline } \\
\text { Supernate }\end{array}$ & $\begin{array}{l}\mathrm{Ce} \\
\mathrm{Cs} \\
\mathrm{Sr} \\
\mathrm{Tc} \\
\mathrm{Y} \\
\mathrm{Cr} \\
\mathrm{Co} \\
\mathrm{Fe} \\
\mathrm{Mn} \\
\mathrm{Zn} \\
\mathrm{Zr} \\
\mathrm{U} \\
\mathrm{Am}\end{array}$ & $\begin{array}{r}139 \\
2323 \\
4660 \\
<0.1 \\
13 \\
<0.1 \\
0.2 \\
36 \\
52 \\
2.6 \\
83 \\
16 \\
47\end{array}$ & $\begin{array}{r}>300 \\
2708 \\
>4500 \\
0.2 \\
16 \\
<0.1 \\
0.4 \\
60 \\
109 \\
3.2 \\
85 \\
30 \\
92\end{array}$ & $\begin{array}{r}>300 \\
3076 \\
>4600 \\
<0.1 \\
24 \\
<0.1 \\
0.4 \\
93 \\
225 \\
3.7 \\
68 \\
43 \\
53\end{array}$ \\
\hline
\end{tabular}


g. SNL/CST 120 Crystalline Silico-Titanate. This absorber sorbs strontium more strongly than cesium from alkaline supernate solution, although cesium also is strongly sorbed. Many other elements are cosorbed at useful levels from this solution.
Although SNL/CST 120 was not studied with aciddissolved sludge or acidified supernate solutions, the fact that only cesium is strongly sorbed on most CSTs from acid-dissolved sludge solution suggests that most non-cesium elements sorbed from alkaline supernate solution could subsequently be eluted with nitric acid, leaving cesium selectively sorbed.

Table 78. SNL/CST 120: Distribution of 14 Elements from Alkaline Supernate Simulant Solution for Hanford Tank 102-SY

\begin{tabular}{lcrrr}
\hline & & \multicolumn{3}{c}{ Kd Value for Specified Time } \\
\cline { 2 - 5 } Solution & Element & $\mathbf{3 0 ~} \mathbf{~ m i n}$ & $\mathbf{2 ~ h}$ & $\mathbf{6 ~ h}$ \\
\hline Alkaline & $\mathrm{Ce}$ & 106 & 230 & 150 \\
Supernate & $\mathrm{Cs}$ & 570 & 786 & 898 \\
& $\mathrm{Sr}$ & $>4400$ & $>4500$ & $>4600$ \\
& $\mathrm{Tc}$ & $<0.1$ & 0.1 & $<0.1$ \\
& $\mathrm{Y}$ & 8.3 & 12 & 21 \\
& $\mathrm{Cr}$ & $<0.1$ & $<0.1$ & $<0.1$ \\
& $\mathrm{Co}$ & 0.1 & 0.5 & 0.2 \\
& $\mathrm{Fe}$ & 52 & 104 & 166 \\
$\mathrm{Mn}$ & 24 & 117 & 215 \\
& $\mathrm{Zn}$ & 7.6 & 7.0 & 9.4 \\
& $\mathrm{Zr}$ & 147 & 283 & 449 \\
& $\mathrm{U}$ & 73 & 77 & 101 \\
& $\mathrm{Am}$ & 15 & 43 & 71 \\
\hline
\end{tabular}


h. SNL/CST 141 Crystalline Silico-Titanate. This absorber sorbs strontium more strongly than cesium from alkaline supernate solution, especially for longer contact times, although cesium also is strongly sorbed. Many other elements are cosorbed at useful levels.
Although SNL/CST 141 was not studied with aciddissolved sludge or acidified supernate solutions, the fact that only cesium is strongly sorbed on most CSTs from acid-dissolved sludge solution suggests that most non-cesium elements sorbed from alkaline solution could subsequently be eluted with nitric acid, leaving cesium selectively sorbed.

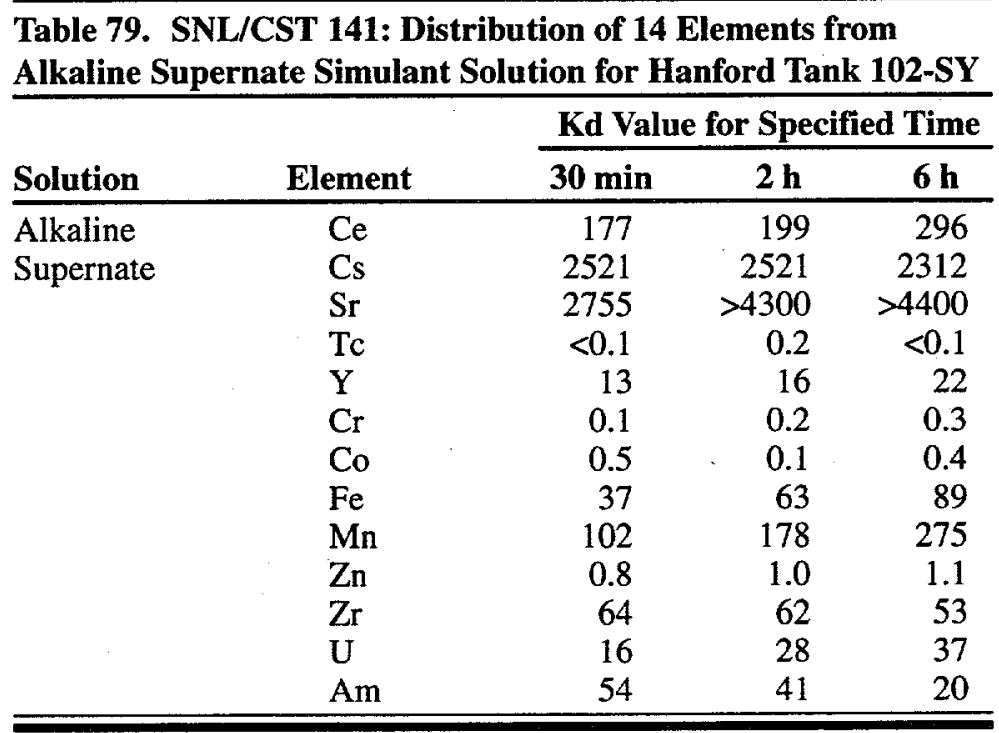


i. SNL/CST 149 Crystalline Silico-Titanate. This absorber sorbs strontium more strongly than cesium from alkaline supernate solution, especially for longer contact times, although cesium also is strongly sorbed. Many other elements are cosorbed at useful levels.

Although SNL/CST 149 was not studied with aciddissolved sludge or acidified supernate solutions, the fact that only cesium is strongly sorbed on most CSTs from acid-dissolved sludge solution suggests that most non-cesium elements sorbed from alkaline supernate solution could subsequently be eluted with nitric acid, leaving cesium selectively sorbed.

Table 80. SNL/CST 149: Distribution of 14 Elements from Alkaline Supernate Simulant Solution for Hanford Tank 102-SY

\begin{tabular}{lccrr}
\hline & & \multicolumn{3}{c}{ Kd Value for Specified Time } \\
\cline { 2 - 5 } Solution & Element & $\mathbf{3 0 ~} \mathbf{~ m i n}$ & $\mathbf{2 ~ h}$ & $\mathbf{6 ~ h}$ \\
\hline Alkaline & $\mathrm{Ce}$ & 797 & 948 & 701 \\
Supernate & $\mathrm{Cs}$ & 1866 & 2402 & 3084 \\
& $\mathrm{Sr}$ & $>9 \mathrm{~K}$ & $>9 \mathrm{~K}$ & $>9 \mathrm{~K}$ \\
& $\mathrm{Tc}$ & 1.5 & 1.5 & 1.4 \\
& $\mathrm{Y}$ & 81 & 77 & 104 \\
& $\mathrm{Cr}$ & 0.6 & 0.6 & 0.7 \\
& $\mathrm{Co}$ & 0.6 & 0.6 & 0.6 \\
& $\mathrm{Fe}$ & 37 & 58 & 94 \\
& $\mathrm{Mn}$ & 69 & 134 & 235 \\
& $\mathrm{Zn}$ & 0.9 & 1.2 & 1.6 \\
& $\mathrm{Zr}$ & 94 & 104 & 95 \\
& $\mathrm{U}$ & 15 & 27 & 39 \\
& $\mathrm{Am}$ & 12 & 11 & 12 \\
\hline \hline
\end{tabular}


j. SNL/HTO Amorphous Hydrous $\mathrm{TiO}_{2}$. As expected, the behavior of hydrous titanium oxide is very different from that of the crystalline silico-titanates. With this absorber, cerium, americium, uranium, yttrium, and zinc all sorb with double-digit $\mathrm{Kd}$ values from acidified supernate solution.
From alkaline supernate solution, the sorption of cesium is very weak, whereas many other elements are sorbed very strongly. SNL/HTO absorber was not studied with aciddissolved sludge solution.

Table 81. SNL/HTO: Distribution of 14 Elements from Two Simulant Solutions for Hanford Tank 102-SY

\begin{tabular}{|c|c|c|c|c|}
\hline \multirow[b]{2}{*}{ Solution } & \multirow[b]{2}{*}{ Element } & \multicolumn{3}{|c|}{ Kd Value for Specified Time } \\
\hline & & $30 \mathrm{~min}$ & $2 \mathrm{~h}$ & $6 h$ \\
\hline Acidified & $\mathrm{Ce}$ & 265 & 320 & 511 \\
\hline \multirow[t]{13}{*}{ Supernate } & Cs & 10 & 8.9 & 8.5 \\
\hline & $\mathrm{Sr}$ & 1.5 & 1.4 & 3.6 \\
\hline & Tc & 1.3 & 1.7 & 4.3 \\
\hline & Y & 53 & 59 & 52 \\
\hline & $\mathrm{Cr}$ & 5.9 & 7.8 & 21 \\
\hline & Co & 0.9 & 0.7 & 0.4 \\
\hline & $\mathrm{Fe}$ & 2.2 & 3.1 & 3.9 \\
\hline & $\mathrm{Mn}$ & 1.2 & 0.3 & 0.4 \\
\hline & $\mathrm{Zn}$ & 18 & 18 & 19 \\
\hline & $\mathrm{Zr}$ & 1.7 & 2.7 & 6.3 \\
\hline & $\mathrm{U}$ & 52 & 69 & 90 \\
\hline & $\mathrm{Pu}$ & 1.5 & 1.8 & 2.4 \\
\hline & Am & 229 & 264 & 168 \\
\hline Alkaline & $\mathrm{Ce}$ & 184 & $>280$ & $>290$ \\
\hline \multirow[t]{12}{*}{ Supernate } & Cs & 1.5 & 1.4 & 1.4 \\
\hline & $\mathrm{Sr}$ & $>4000$ & $>4100$ & $>4200$ \\
\hline & $\mathrm{Tc}$ & $<0.1$ & $<0.1$ & $<0.1$ \\
\hline & $\mathbf{Y}$ & 37 & 68 & 93 \\
\hline & $\mathrm{Cr}$ & $<0.1$ & 0.3 & 0.3 \\
\hline & Co & 0.8 & 0.8 & 1.0 \\
\hline & $\mathrm{Fe}$ & 4235 & $>7 \mathrm{~K}$ & $>26 \mathrm{~K}$ \\
\hline & Mn & 1969 & 4777 & $>9 \mathrm{~K}$ \\
\hline & $\mathrm{Zn}$ & 4625 & $>11 \mathrm{~K}$ & $>25 \mathrm{~K}$ \\
\hline & $\mathrm{Zr}$ & 1037 & 2220 & 4276 \\
\hline & $\bar{U}$ & 2127 & $>9 \mathrm{~K}$ & $>17 \mathrm{~K}$ \\
\hline & $\mathrm{Am}$ & 200 & 510 & 779 \\
\hline
\end{tabular}




\section{ELUTION CONSIDERATIONS}

The purpose of this study was to evaluate a large number of candidate absorbers and identify those that show promise for sorbing specific elements from HLW stored in Hanford Tank 102-SY. Although elution was not a part of the experimental procedure, we recognize that elution of the sorbed species from the absorbers is essential if the selected absorbers are to be reused in a process application. We plan to investigate the elution characteristics of selected absorbers in a later phase of this study.

We can, however, offer some general comments about elution. Elution often can be achieved by acidity changes, oxidation state changes, complexation, or displacement. Because the present study reports $\mathrm{Kd}$ values for sorption from three simulant solutions that range from acidic to basic, it often indicates whether acidity changes might facilitate the elution of a given species. For example, the fact that most Sandia CSTs strongly sorb both strontium and cesium from alkaline solution, but only cesium from acidic solution, suggests that the cosorbed strontium might elute with nitric acid, which would leave cesium selectively sorbed on the CST.

For those elements in which only one oxidation state strongly sorbs on an absorber, conversion to a different oxidation state may achieve elution. In other cases, the sorbed species may form a strong complex with specific complexants, which can be used as eluting agents. Finally, sorbed species sometimes can be displaced by loading the absorber to capacity with another, more strongly sorbed, ion.

Because elution operations usually generate additional waste, we plan to emphasize waste minimization in the elution options we evaluate in later studies. One option worth considering is that of not eluting the sorbed species from the absorber. This option is attractive in the case of the absorbers developed by Sandia and Texas A\&M because cesium appears to be strongly and irreversibly sorbed on crystalline silico-titanates. Moreover, silico-titanates provide an inorganic matrix that could be pressed into dense pellets suitable for long-term storage. In this case, elution may be unwise and unnecessary.

\section{FUTURE STUDIES}

This screening study met its objective of identifying specific absorbers that appear capable of partitioning targeted elements from Hanford Tank 102-SY. The best absorbers and promising new materials are being evaluated with simulant compositions that represent other
Hanford tanks, especially those scheduled to be processed first. The 14 elements for which we acquired sorption data in this study will be reviewed to determine whether some should be deleted and others added in future measurements. We plan to continue using radioactive tracers, which provide a rapid, reliable, and inexpensive way to obtain large amounts of distribution data.

Because even the best simulant solutions cannot accurately represent the contents of any HLW storage tank, we look forward to obtaining and testing solutions from the Hanford waste tanks. Only by testing actual waste solutions can we obtain sorption data that are sufficiently reliable to permit the design of full-scale partitioning processes to begin. We recognize that even tests with actual wastes will not be conclusive because the contents of the Hanford tanks vary greatly and that individual tanks exhibit large heterogeneities in their horizontal and vertical compositions. However, as we obtain sorption data for the most promising absorbers with samples from various HLW tanks, we hope to identify absorbers that sorb the target elements well from a wide variety of waste solutions.

Finally, chemical and radiation stabilities are important factors to consider when selecting materials for processing radioactive waste solutions. The long-term chemical stability and the effect of radiation on the most promising absorbers from our present and future studies should be determined (if such information is not already available) before these absorbers are considered for use in large-scale recovery processes

\section{CONCLUSIONS}

Our screening study of many absorbers from realistic simulants has met the initial objective of identifying many promising absorbers. Moreover, because we measured the sorption of so many different elements, our study provides important selectivity information that specifies which unwanted elements are most likely to compete for absorber sites. Based on our study, it appears that many of the separations required for processing HLW from Hanford Tank 102-SY, and perhaps other underground storage tanks as well, can be achieved using available absorbers and existing, proven technology.

\section{A. Experimental Procedure}

Radioactive tracers, as used in our study, provide a fast, reliable, and cost-effective way to obtain large amounts of distribution data. 
Follow-on studies should measure the distributions of key elements from other Hanford waste tank compositions onto the most promising absorbers identified by this study.

\section{B. Individual Absorbers}

1. The performance of the 63 absorbers tested with these complex, high-salt media is often much different from the performance reported for similar absorbers tested in relatively clean systems.

2. None of the tested absorbers sorbs strontium well from acid-dissolved sludge or acidified supernate solutions.

3. Low-cost partitioning agents such as Aliquat ${ }^{\mathrm{TM}} 336$, Cyanex ${ }^{\mathrm{TM}}$ 923, and Reillex ${ }^{\mathrm{TM}} \mathrm{HPQ}$ often outperform other materials whose cost is many times higher.

4. Certain extractants, such as LIX-1010 and LIX-54, sorb americium much better than cerium and yttrium from acidified supernate solution. This large difference in sorption suggests that a lanthanide/trivalent actinide group separation may be attainable. An earlier study ${ }^{5}$ estimated that, if the lanthanide elements could be eliminated from the trivalent actinide fraction before vitrification, 5000 fewer vitrified HLW logs would be required, with a consequent saving of approximately $\$ 5$ billion.

5. Experimental anion exchange resins under development at Los Alamos, referred to as JSK resins in this report, offer major advantages over the best previously known resins for removing plutonium from HLW media. Development of these resins should continue until a commercial version is produced.

6. The silico-titanate absorbers from Sandia show outstanding sorption of cesium from all three simulant solutions. Because the sorption of cesium on silicotitanates appears to be irreversible, this material should be considered for interim storage of radioactive waste, incorporation into HLW glass, or as a final waste form.

\section{Individual Elements}

1. Measured $\mathrm{Kd}$ values for strontium are low on all absorbers for sorption from dissolved sludge and acidified supernate solutions. Because ${ }^{90} \mathrm{Sr}$ is a major biological hazard, near-term efforts should focus on identifying or developing suitable absorbers for removing strontium from acidic HLW solutions.
2. Measured Kd values for sorption of cobalt and zinc from acid-dissolved sludge solution are low on all absorbers. If there is need to remove these elements from similar acidic waste solutions, other absorbers or extractants must be identified or developed.

3. Iron, manganese, and chromium show low $\mathrm{Kd}$ values for sorption from acidic solutions on most of the absorbers evaluated. Because these elements are present at significant concentrations in many Hanford waste tanks, however, even low Kd values could result in sufficiently high loadings on some absorbers to decrease their capacity for sorbing other elements. All reported $\mathrm{Kd}$ values reflect realistic competition from these major matrix elements and, therefore, reflect values that might be obtained with actual waste solutions.

4. Elements for which good to excellent sorption from simulated acid-dissolved sludge has been demonstrated are cerium, cesium, technetium, zirconium, uranium, and plutonium. Elements that show low sorption from this solution are strontium, yttrium, chromium, cobalt, iron, manganese, zinc, and americium.

5. Elements for which good to excellent sorption from acidified supernate solution has been demonstrated are cerium, cesium, technetium, yttrium, zinc, uranium, and americium. Elements that show less than desirable sorption from acidified supernate solution are strontium, chromium, cobalt, iron, manganese, zirconium, and plutonium.

6. Elements for which good to excellent sorption from alkaline supernate simulant solution has been demonstrated are cerium, cesium, strontium, yttrium, zinc, iron, manganese, zirconium, uranium, and americium. Elements that show low to moderate sorption from alkaline supernate solution are chromium and cobalt.

\section{Elution}

Although elution was not a part of our study, by measuring $\mathrm{Kd}$ values from acidic, slightly acidic, and basic solutions, we often provide a basis for predicting whether a change in acidity might elute a given element from a specific absorber.

For example, most CSTs strongly sorb both cesium and strontium from alkaline supernate solution. The fact that only cesium is sorbed strongly from acid-dissolved sludge solution, however, indicates that the strontium sorbed from alkaline supernate solution could be eluted with nitric acid, leaving cesium selectively sorbed. 


\section{ACKNOWLEDGMENTS}

This study was supported by the Hanford Tank Waste Remediation System (TWRS) program of DOE/ Richland Operations Office; the TWRS Technology Development Program Office (TDPO) of Westinghouse Hanford Company; and the Hanford Program Office, EM-36, of the Office of Environmental Restoration and Waste Management, U.S. Department of Energy.

Clifford Mills of the Nuclear Materials Process Technology group (NMT-2) at Los Alamos designed the tip-filter system that allowed disposable hypodermic syringes to be successfully used as container-dispensers during our many hundreds of dynamic contact experiments.

Frank McGarvey of Sybron Chemical Co. provided samples of macroporous anion exchange resins with trimethyl, triethyl, tripropyl, and tributyl amine functional groups.

Jane Bibler of Westinghouse Savannah River Company provided the sample of SRS resorcinol/formaldehyde resin (batch BSC-187).

Dennis Fennelly of UOP Molecular Sieves provided samples of Ionsiv TM TIE-96 for evaluation.

Elmer Klavetter and Norman Brown of Sandia National Laboratories provided samples of crystalline silico-titanate (SNL/CST) absorbers and hydrous titanium oxide (SNL/HTO) absorber.

Ferdinand Sebesta, Department of Nuclear Chemistry, Czech Technical University, Prague, Czech Republic, prepared and provided samples of nine polyacrylonitrile-matrix composite absorbers for our evaluation.

Jerzy Narbutt, Department of Radiochemistry, Institute of Nuclear Chemistry and Technology, Warsaw, Poland, prepared and provided samples of two phenolsulfonic-formaldehyde-matrix composite absorbers for our evaluation.

Stephen Yarbro and Gordon Jarvinen of the Nuclear Materials Process Technology group (NMT-2) at Los Alamos provided valuable advice and suggestions during numerous technical discussions.

Stephen Agnew of the Spectroscopy and Biochemistry group (CST-14) at Los Alamos provided much detailed tank composition data, based on reconstruction from historical records and many useful technical suggestions.

Norman Brown of Sandia National Laboratories provided valuable advice and suggestions during numerous technical discussions and also peer-reviewed this manuscript.
We especially appreciate the assistance and cooperation of many members of the Separations and Radiochemistry Group, the Nuclear Chemistry and Analysis Group, and the Medical Radioisotopes and Reactor Applications Group at Los Alamos National Laboratory.

\section{REFERENCES}

1. J. L. Ryan and E. J Wheelwright, "Recovery and Purification of Plutonium by Anion Exchange," Ind. Eng. Chem. 51(1), 60-65 (1959).

2. W. L. Poe, A. W. Joyce, and R. I. Martens, "Np-237 and Pu-238 Separation at the Savannah River Plant," I\&EC Proc. Design Devel. 3(4), 314-322 (1964).

3. J. A. Kelly, "Ion Exchange Process for Separating Americium and Curium from Irradiated Plutonium," Savannah River Laboratory report DP-1308 (1972).

4. S. L. Yarbro, W. A. Punjak, S. B. Schreiber, S. L. Dunn, G. D. Jarvinen, S. F. Marsh, N. G Pope, S. F. Agnew, E. Birnbaum, and K. W. Thomas, and E. A. Ortic, "Tank 102-SY Remediation ProjectFlowsheet and Conceptual Design," Los Alamos National Laboratory report LA-12701-MS (January 1994).

5. J. L. Straalsund, J. L. Swanson, E. G. Baker, J. J. Holmes, E. O. Jones, and W. L. Kuhn, "Clean Option: An Alternative Strategy for Hanford Tank Waste Remediation: Volume 1. Overview," Pacific Northwest Laboratory report PNL-8388, Vol. 1 (December 1992).

6. R. K. Scheele and M. K. Peterson, "Results of the Characterization of Samples of Waste from DoubleShell Tank 102-SY," Westinghouse Hanford Company internal letter report (January 1990).

7. S. F. Agnew, "Preliminary Estimation of SY-102 Based on Tank History," Los Alamos National Laboratory internal letter report INC-14:93sfa1645 (May 18, 1993).

8. G. J. Lumetta and J. L. Swanson, "Pretreatment of Plutonium Plant (PFP) Sludge: Report for the Period October 1990-March 1992," Pacific Northwest Laboratory report TWRSPP-92-011 Draft (June 9, 1992). 
9. R. Gunnink and J. B. Niday, "Computerized Quantitative Analysis by Gamma-Ray Spectrometry. Vol. 1. Description of the Gamanal Program," Lawrence Livermore National Laboratory report UCRL-51061, Vol. 1 (March 1972).

10. T. M. Benjamin, Nuclear Chemistry and Analysis Group, Los Alamos National Laboratory, personal communication, April 1994.

11. F. X. McGarvey and R. Gonzales, "Ion Exchange Studies on Strongly Basic Anion Exchange Resins Prepared with Tertiary Amines of Varying Molecular Weight," in Ion Exchange Advances, Proceedings of IEX '92, M. J. Slater, Ed. (Elsevier Science Publishing Ltd., Essex, England, 1992), pp. 97-103.

12. S. F. Marsh, "The Effect of Triethyl, Trimethyl, Tripropyl, and Tributyl Amine Functional Groups in Strong-Base Anion Exchange Resin on the Sorption of Pu(IV) from Nitric Acid," in Ion Exchange Processes: Advances and Applications, A. Dyer, M. J. Hudson, and P. A. Williams, Eds. (Royal Society of Chemistry, Cambridge, England, 1993), pp. 81-89.
13. F. Sebesta, A. Motl, and J. John, "Composite IonExchangers, Their Development and Use," in Workshop of the Czech Technical University in Prague, INIS-mf-13201 or CONF-9201108 (January 1992), pp. 25-26.

14. J. Narbutt, A. Bilewicz, and B. Bartos, "Composite Ion Exchangers for Radiocaesium Removal from Nuclear Reactor Wastes," in Proc. Int. Symp. on Management of Low and Intermediate Level Radioactive Wastes, CONF-880510, v-2 (Stockholm, May 16-20, 1988).

15. T. M. Suzuki, "Porous Spherical Resins Loaded with Hydrous Metal Oxides-Preparation and the Ion Exchange Properties," in New Developments in Ion Exchange; Materials, Fundamentals, and Applications, Proc. Int. Conf. on Ion Exchange, ICIE '91, M. Abe, Ed. (Elsevier, Amsterdam-OxfordNew York-Tokyo, 1992), pp. 181-186. 